\title{
Förderung selbstregulierten Lernens im Unterricht: \\ Herausforderungen, Ansatzpunkte, Chancen
}

\author{
Dissertation
}

zur Erlangung des mathematisch-naturwissenschaftlichen Doktorgrades

"Doctor rerum naturalium"

der Georg-August-Universität Göttingen

vorgelegt von

Andju Sara Labuhn

aus Coesfeld

Göttingen 2008 
Referent: Prof. Dr. Marcus Hasselhorn

Korreferentin: Prof. Dr. Susanne Bögeholz

Tag der mündlichen Prüfung: 20.06.2008 
„Ich bin zu der Ansicht gekommen,

dass die einzigen Lerninhalte, die Verhalten signifikant beeinflussen,

selbst entdeckt, selbst angeeignet werden müssen.“

Carl Rogers,

Psychologe und Psychotherapeut 


\section{Danke}

In den vergangenen Jahren haben viele Menschen zum Entstehen dieser Dissertation beigetragen. Alle haben mich auf ihre Weise unterstützt und es mir ermöglicht meinen Weg zu finden und zu gehen.

Ein besonderer Dank gilt Prof. Dr. Marcus Hasselhorn. Er hat während meiner Promotionszeit meine Arbeit zielgerichtet, jedoch meine Kreativität nie einschränkend, betreut. Ich danke ihm für das stete Vertrauen in meine Fähigkeiten, das mir besonders in Momenten, in denen ich selbst gezweifelt habe, sehr geholfen und mich motiviert hat.

Ebenso herzlich bedanke ich mich bei meiner Zweitbetreuerin Prof. Dr. Susanne Bögeholz für ihre Unterstützung und die hilfreichen Anregungen, die meinen Blick aus einer interdisziplinären Perspektive geschult und meine Arbeit bereichert haben.

Prof. Dr. Barry Zimmerman sowie Adam und Christoph danke ich herzlichst für großartige und lehrreiche Monate an der City University of NewYork - für die Gastfreundschaft und viele anregende Diskussionen. Die Zeit in New York hat meine Arbeit sehr geprägt und meine Begeisterung geweckt.

Meine Dissertation entstand im Rahmen des Graduiertenkollegs „Passungsverhältnisse schulischen Lernens" (GRK 1195). Hier traf ich auf eine Gruppe aufgeschlossener, interessierter, kommunikativer und hilfsbereiter Menschen. Ohne meine lieben Mitstipendiat(inn)en wäre meine Promotionszeit nicht so erfüllend gewesen. Teil dieses GRKs zu sein hat mir viel bedeutet. Ein großer Dank geht an Euch. Ich nehme viele schöne Erinnerungen mit.

Besonders danke ich Ulrike. Wir haben viel Zeit miteinander verbracht, haben inhaltlich diskutiert, organisiert, Gedanken ausgetauscht. Doch weit darüber hinaus habe ich sehr viel Unterstützung erfahren - immer im richtigen Moment. Ulrike, Du hast mir viel Mut gemacht und mich bestärkt, meine Ziele zu erkennen und zu verfolgen. Nicht zuletzt zu dieser Arbeit hast Du Deinen wertvollen Beitrag geleistet. Danke!

Nicht zu vergessen sind meine lieben Freunde Bastian und Matthias, die immer für mich da waren und für die nötige Abwechslung gesorgt haben. Danke für den steten Rückhalt und die zuverlässige Hilfe bei allen möglichen und unmöglichen Dingen - und dass Ihr meinen Dickkopf ertragen habt. 
Johanna, Rebekka und Kurt danke ich ebenfalls für die wundervolle langjährige Freundschaft, für lebendige Gespräche, gemeinsame kreative Ideen und ganz viel Lebensfreude. Darüber hinaus haben sie mit ihren kompetenten Anmerkungen sehr zum Gelingen dieser Arbeit beigetragen.

Sara, David, Alexander, Annika, Anja, Karin, Fritz, Bruno, Gudrun, Günther, Denia, Rainer und Bärbel - Danke, dass Ihr von Anfang an meinen Weg begleitet und an mich geglaubt habt.

Sascha, Dir danke ich für die liebevolle Geduld, die Du in den vergangenen Monaten für mich aufgebracht und den Halt, den Du mir gegeben hast.

Ganz besonders danke ich meinen lieben Eltern für ihre grenzenlose Unterstützung. Ihr habt an mich geglaubt und mir Türen geöffnet. Eure Liebe hat es mir ermöglicht da zu sein, wo ich nun stehe. Auch meinem Bruder Cord Henning danke ich für seine liebevolle Begleitung, seine Anerkennung und vor allem für sehr viel Inspiration. Mein herzlicher Dank gilt außerdem meiner Duisburger Familie: Margret, Stefan und Manuela mit Lenny. Ich danke zutiefst Waltraud und Theo Labuhn, die leider nicht mehr unter uns sind, für ihre bedingungslose Liebe und viele wunderschöne Erinnerungen an meine Kindheit.

Schließlich möchte ich mich bei all meinen Freund(inn)en bedanken, die ich hier nicht namentlich genannt habe. Ihr alle habt mir stets Halt und Wärme gegeben.

Ich weiß mein Glück zu schätzen. Ich danke Euch allen.

Göttingen im Juli 2008,

Andju Sara Labuhn 


\section{Inhalt}

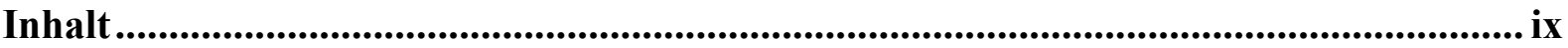

Tabellenverzeichnis .......................................................................................................................... xi

Abbildungsverzeichnis............................................................................................................. xiii

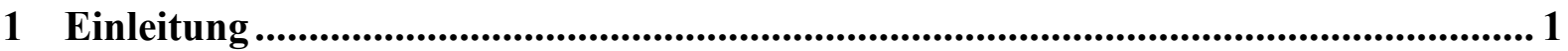

2 Selbstreguliertes Lernen aus sozial-kognitiver Perspektive .............................................. 5

2.1 Die Bedeutung der sozial-kognitiven Grundannahmen für das Verständnis von

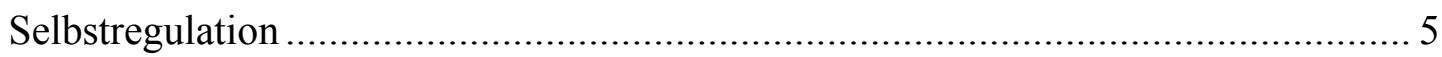

2.2 Die Theorie selbstregulierten Lernens nach Zimmerman ........................................ 9

2.2.1 Selbstreguliertes Lernen als zyklischer Prozess ..................................................................... 9

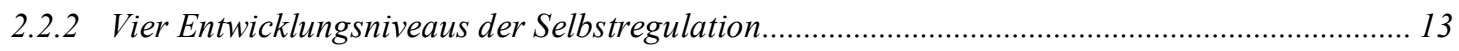

3 Selbstregulationsförderung unter sozial-kognitiven Gesichtspunkten ......................... 17

3.1 Implikationen der sozial-kognitiven Theorie für schulisches Lernen........................ 17

3.2 Ansätze zur Förderung selbstregulierten Lernens................................................. 22

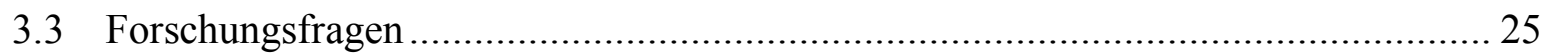

4 Integration der Selbstregulationsförderung in den naturwissenschaftlichen

Fachunterricht (Studie 1) ............................................................................................... 27

4.1 Lernförderung durch Anregung der Selbstregulation im naturwissenschaftlichen

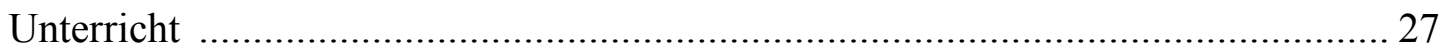

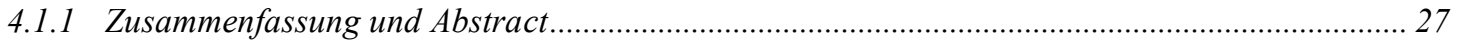

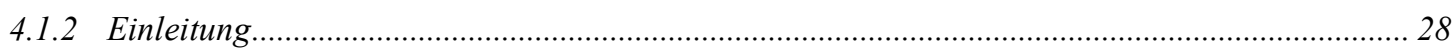

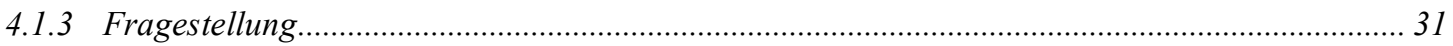

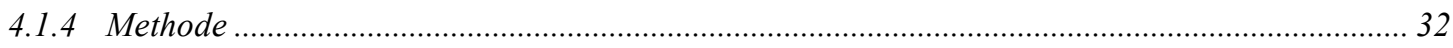

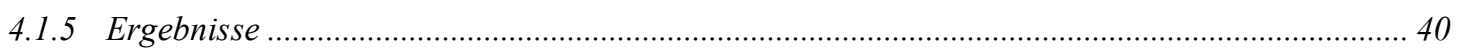

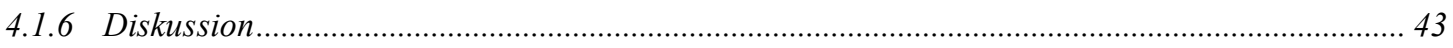

4.2 Herausforderungen für Trainingsansätze im Schulkontext ................................... 48

4.3 Selbstregulationsförderung in einer Biologie-Unterrichtseinheit - langfristige und differentielle Wirksamkeit ...................................................................... 52

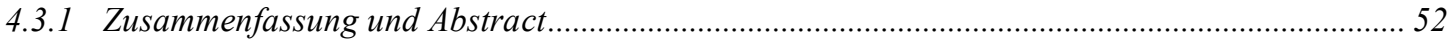

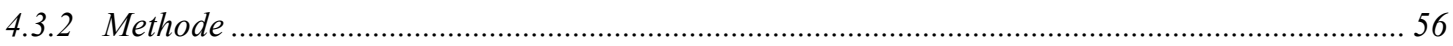

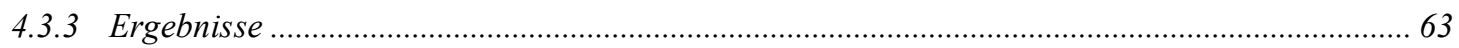

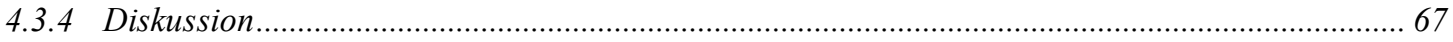


4.4 Kritisches Resümee zu Studie 1 ....................................................................... 73

4.4.1 Praktische Grenzen der Unterrichtsintervention ........................................................................ 73

4.4.2 Methodische Grenzen der Wirksamkeitsevaluation .................................................................. 79

5 Mechanismen der Wirksamkeit instruktionalen Handelns zur Förderung

selbstregulierten Lernens .............................................................................................. 85

5.1 Überlegungen zur Selbstregulationsforschung auf Prozessebene............................ 85

5.2 Prozessorientierte Forschung im Unterrichtskontext: Zur Bedeutung von Bewertungsstandards und Feedback im Selbstregulationsprozess......................... 88

5.3 Selbstwirksamkeitserwartungen, Metakognition und die Frage der "Kalibrierung"

6 Enhancing students' self-regulation and mathematics performance: The influence of feedback and self-evaluative standards (Studie 2) ............................. 95

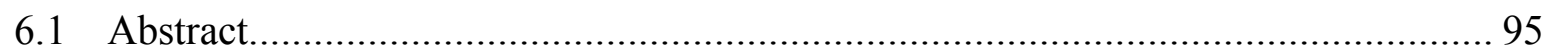

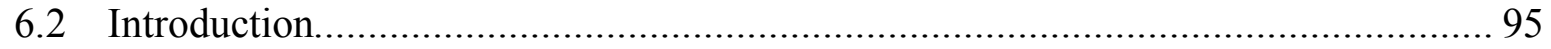

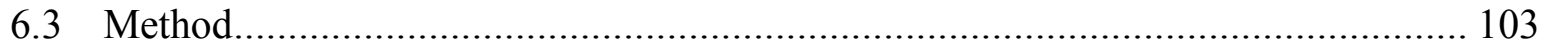

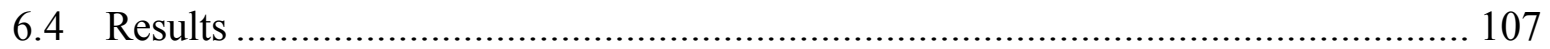

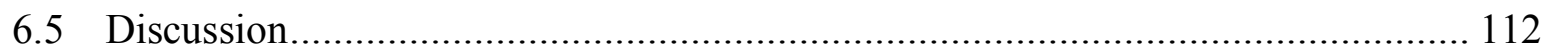

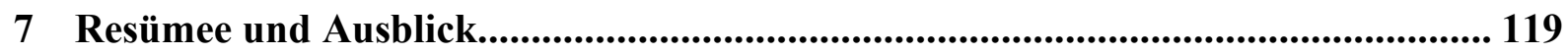

7.1 Zusammenfassung und Diskussion .............................................................. 119

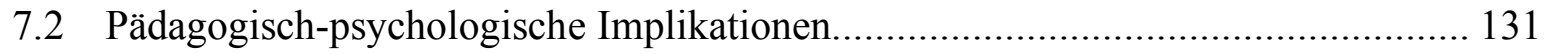

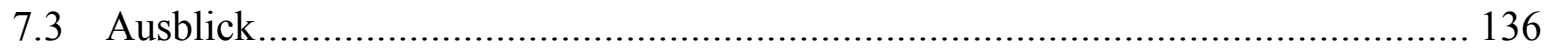

Zusammenfassung ......................................................................................................... 139

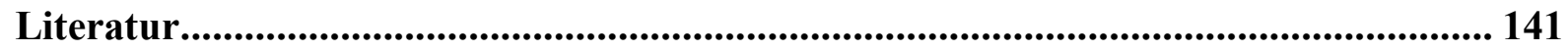

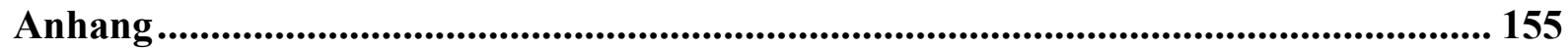

Anhang A Messinstrument von Studie 1 ................................................................. 157

Anhang B Instruktion für die experimentellen Gruppen in Studie 2 ............................. 171

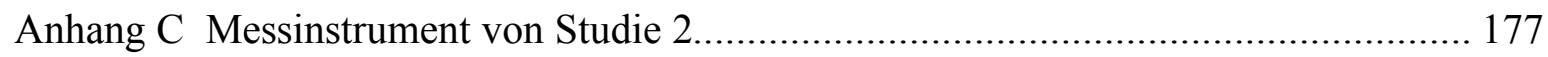

Anhang D Aufgaben der vier Übungsdurchgänge in Studie 2 ................................. 185 


\section{Tabellenverzeichnis}

Tabelle 2.1. Entwicklungsniveaus der Selbstregulationsfähigkeit nach Zimmerman.

Tabelle 3.1. Elemente der sozial-kognitiven Theorie, Implikationen und praktische

Beispiele ....

Tabelle 4.1. Unterrichtseinheit Ernährung und Umsetzung der integrierten SRL-

Förderung.

Tabelle 4.2. Skalen des Selbstregulations-Fragebogens und Reliabilitäten in der

Ursprungsstudie sowie in der vorliegenden Studie 38

Tabelle 4.3. Itembeispiele für die lehrzielorientierten Tests.

Tabelle 4.4. Mittelwerte (und Standardabweichungen) der Selbstregulationsskalen für Trainings- und Kontrollgruppe.

Tabelle 4.5. Mittlere z-Werte (und Standardabweichungen) der fachbezogenen Tests

für Trainings- und Kontrollgruppe.

Tabelle 4.6. Inhalte der Unterrichtseinheit Ernährung und Elemente der integrierten

SRL-Förderung 58

Tabelle 4.7. Itembeispiel für den lehrzielorientierten Test zum Thema Ernährung. 62

Tabelle 4.8. Mittelwerte (und Standardabweichungen) der Selbstregulationsskalen für Trainings- und Kontrollgruppe für Prätest, Posttest und Follow-up

Tabelle 4.9. Mittelwerte (und Standardabweichungen) der fachbezogenen Tests für

Trainings- und Kontrollgruppe

Tabelle 5.1. Gegenüberstellung der globalen und kontextspezifischen Erfassung von

Selbstregulationsvariablen. 86

Tabelle 5.2. Messung der Genauigkeit von Selbstwirksamkeitserwartung und Selbsteinschätzung 94

Tabelle 6.1. Math task examples 103

Tabelle 6.2. Dependent Measure Means and Standard Deviations for Control and Each Experimental Group in Complete Sample and Overconfident

Students 109

Tabelle 6.3. Correlations Among Dependent Measures 112

Tabelle 7.1. Gegenüberstellung zentraler Merkmale von Studie 1 und Studie 2. 130

Tabelle 7.2. Zentrale Befunde und Implikationen. 134 


\section{Abbildungsverzeichnis}

Abbildung 2.1. Wechselseitiger Einfluss der Teilbereiche der Selbstregulation nach Zimmerman (1989a) 7

Abbildung 2.2. Phasenmodell des selbstregulierten Lernens nach Zimmerman und Campillo (2003) in der deutschen Übersetzung von Hasselhorn und Labuhn (2008)

Abbildung 4.1. Interaktion Gruppe x Messzeitpunkt; Subskalen und Gesamtskala

SRL 42

Abbildung 4.2. Beispiel für das Ergebnismuster eines Matthäuseffekts 50

Abbildung 4.3. a) Haupteffekt Migrationshintergrund im fachbezogenen Posttest und

b) Interaktion Gruppe x Migrationshintergrund im fachbezogenen Follow-up Test

Abbildung 5.1. Kontextspezifische Messung zur Analyse der

Einschätzungsgenauigkeit innerhalb des Selbstregulationszyklus 93 


\section{$1 \quad$ Einleitung}

In einer Umwelt, in der Wissen komplexer und auf immer vielfältigere Weise verfügbar wird, sind Menschen mit einer Fülle von Informationen konfrontiert, deren Qualität stark variiert. Das Vermögen, Informationen kritisch zu reflektieren, sie mit vorhandenen Kenntnissen abzugleichen und einen eigenen Standpunkt zu beziehen, gewinnt einen immer höheren Stellenwert. Sowohl bei der Bewertung von Fakten (siehe dazu auch Bögeholz \& Barkmann, 2005), öffentlichen Meinungen und Ansichten als auch beim Generieren neuer, weiterführender Fragen kommt der Fähigkeit zur Regulation der Informationsaufnahme eine große Bedeutung zu. Im Zuge der raschen gesellschaftlichen und technologischen Fortentwicklung stehen Menschen darüber hinaus vor immer neuen Aufgaben und wissenschaftlichen Herausforderungen, für deren Bewältigung der Erwerb entsprechender Fähigkeiten verlangt wird. Interdisziplinarität gewinnt an Bedeutung, Professionen differenzieren sich aus und verknüpfen sich, und den sogenannten „key competencies“ (Schlüsselkompetenzen) wird große Relevanz beigemessen. Die Bereitschaft, Verantwortung für den eigenen - lebenslangen - Lernprozess zu übernehmen, ist neben sozialen Aspekten eine entscheidende Bedingung für erfolgreiche persönliche und berufliche Entwicklung.

Die Vermittlung individueller Voraussetzungen für lebenslanges Lernen sollte daher einen zentralen Bestandteil von Bildung und Erziehung darstellen (Zimmerman, 2002). Sie beginnt optimaler Weise bereits im frühen Kindesalter im Elternhaus und setzt sich mit dem Schuleintritt fort. Der Schule kommt dabei eine besonders wichtige Funktion zu: Sie stellt einen Ort dar, an dem alle Kinder die Möglichkeit erhalten sollten Fähigkeiten zu erwerben, die ihnen eine Entwicklung hin zu eigenständigen und selbstverantwortlichen Individuen erlauben, die sich den Ansprüchen einer sich im ständigen Wandel befindlichen Gesellschaft gewachsen sehen. Ein zentraler Aspekt von Schulbildung sollte daher sein, jungen Menschen zu vermitteln, wie sie Verantwortung für die eigene (Schul-) Leistungsentwicklung und die Aneignung neuer Fähigkeiten und Fertigkeiten übernehmen können und sie darin zu unterstützen, dieses Wissen im Schulkontext und darüber hinaus anzuwenden (Zimmerman, 2006). Die Entwicklung der Situation vieler Schulen in der Bundesrepublik - steigende Schülerzahlen pro Klasse und häufig zu wenig und in der Folge überlastetes Lehrpersonal - hebt die Notwendigkeit selbstständig und selbstverantwortlich Lernende zu fördern hervor. Wird in verschiedenen Kontexten gern von einer „multikulturellen Gesellschaft“ gesprochen, muss konsequenterweise auch der Heterogenität von Lerngruppen Rechnung getragen werden: Sol- 
len Kinder mit ganz unterschiedlichen sozialen und kulturellen Hintergründen erfolgreich gemeinsam lernen, so benötigen sie Lehrkräfte, die nicht nur für die individuellen Stärken und Schwächen der Lernenden sensibel, sondern gleichzeitig in der Lage sind, ein Bewusstsein für solche Fähigkeiten und Grenzen auch bei den Schüler(inne)n selbst aufzubauen. Die aktive Mitgestaltung des eigenen Lernprozesses macht Schüler(innen) unabhängiger von äußeren Bedingungen wie z.B. der Größe der Lerngruppe und der Kapazität von Lehrkräften, stets adäquat auf die individuellen Bedürfnisse aller Lernenden einzugehen.

Aus einer sozial-kognitiven Perspektive, die in der vorliegenden Arbeit hauptsächlich eingenommen wird, hängt jene Bereitschaft zur Verantwortungsübernahme seitens der Schüler(innen) von der Anwendung zentraler Selbstregulationsprozesse sowie ihrer Überzeugung hinsichtlich des Nutzens dieser Prozesse ab (Zimmerman, 2006). Zimmerman (2000, S. 14) definiert selbstreguliertes Lernen als „,...self-generated thoughts, feelings, and actions that are planned and cyclically adapted to the attainment of personal goals". Selbstregulation ist damit ein fortlaufender Prozess, in dessen Rahmen Gedanken, Gefühlen und Handlungen auf individuelle Ziele abgestimmt werden (vgl. auch Hasselhorn \& Labuhn, 2008). Selbstreguliert Lernende werden als Personen beschrieben, die sich bewusst darüber sind, ob und in welchem Maße sie eine bestimmte Fähigkeit oder Wissen auf einem bestimmten Bereich besitzen oder nicht (Zimmerman, 1990). Im Gegensatz zu passiv Lernenden suchen selbstreguliert Lernende aktiv nach Informationen, wenn sie feststellen, dass sie diese benötigen um Aufgaben zu bewältigen oder Fertigkeiten und Fähigkeiten zu erwerben. Stoßen sie auf Schwierigkeiten, zum Beispiel in Form von ungünstigen Lernbedingungen, überforderten Lehrkräften oder schwer verständlichen Schulbüchern, suchen sie eigenständig nach Möglichkeiten, ihre Lernziele trotzdem zu erreichen. Selbstreguliert Lernende betrachten die Aneignung von Fähigkeiten und Fertigkeiten als einen systematischen und kontrollierbaren Prozess und übernehmen als eine Konsequenz dieser Sichtweise größere Verantwortung für schulische Leistungen (Borkowski, Carr, Rellinger \& Pressley, 1990; Zimmerman \& Martinez-Pons, 1986, 1990).

Dieses sozial-kognitive Verständnis von Lernen und schulischer Leistung hat weit reichende Implikationen für die Interaktion von Lehrkräften und Lernenden sowie für die Gestaltung von Unterricht. Individuen wird das Vermögen zugeschrieben, aktiv auf metakognitiver, motivationaler und behavioraler Ebene den eigenen Lernprozess zu gestalten (z.B. Zimmerman, 2000, 2002). Wie Zimmerman (1990, S. 4) treffend formuliert: „, This perspective shifts the focus of educational analyses from students' learning ability and environments as ,fixed' entities to their personally initiated processes and responses designed to improve their ability and their environments for learning “. Das Initiieren und die unterstützende Begleitung 
solcher individuellen Prozesse werden aus dieser Sicht zu zentralen Aufgaben von Erziehung und Unterricht. Das bedeutet auch, Schüler(inne)n nicht nur zu vermitteln, dass sie Verantwortung für das eigene Lernen übernehmen sollen, sondern in erster Linie, wie sie die nötige Kontrolle erlangen und für sich nutzbar machen können.

Ziel der vorliegenden Dissertation ist es, verschiedene Ansätze der Förderung selbstregulierten Lernens im Schulkontext und insbesondere im regulären Unterricht aufzuzeigen und ihre spezifischen Herausforderungen und Chancen zu diskutieren. Dabei kommt der Verknüpfung von fachbezogenem Lernen und der Vermittlung von Selbstregulationsfähigkeiten eine besondere Bedeutung zu. Die Überprüfung der Wirksamkeit verschiedener Ansätze der Förderung erfolgt in der vorliegenden Arbeit am Beispiel von mathematischnaturwissenschaftlichen Fächern. In Studie 1 werden Komponenten der Selbstregulationsförderung in eine Biologie-Unterrichtseinheit ${ }^{1}$ zum Thema Ernährung integriert. Die facettenreichen Inhalte dieses Faches und der starke, originäre Bezug des Ernährungsthemas zur Lebenswelt der Schüler(innen) eignen sich besonders gut für die direkte Verknüpfung mit verschiedenen Selbstregulationselementen und deren Umsetzung im regulären Unterricht. In Studie 2 geht es dagegen um die fokussierte Betrachtung einzelner Subprozesse der Selbstregulation. Für deren genaue Untersuchung bieten sich stark strukturierte, strategiebezogene Aufgaben an. Den fachlichen Schwerpunkt dieser zweiten Studie bildet daher die Mathematik. Der folgende Abschnitt gibt einen Überblick über die Einordnung der beiden genannten Studien in die vorliegende Arbeit und stellt die Inhalte der einzelnen Kapitel kurz vor.

In Kapitel 2 wird mit der Betrachtung selbstregulierten Lernens aus sozial-kognitiver Perspektive der theoretische Rahmen der Arbeit dargestellt. Daran anknüpfend werden in Kapitel 3 die sich aus dem theoretischen Hintergrund ergebenden Implikationen für die Förderung selbstregulierten Lernens abgeleitet. Dieses Kapitel schließt mit der Formulierung der Forschungsfragen für den empirischen Teil der Arbeit. Kapitel 4 beinhaltet mit Studie 1 den ersten Teil der Empirie zur Beantwortung der Forschungsfragen bezüglich der Integration der Selbstregulationsförderung in den naturwissenschaftlichen Fachunterricht. Im Mittelpunkt stehen eine Interventionsstudie sowie die Überprüfung der Wirksamkeit des Ansatzes, des möglichen Transfers von erworbenen Fertigkeiten und der langfristigen Wirkung auf Selbstregulation und Leistungsentwicklung. Ein wichtiges Ziel des Ansatzes stellt dabei die Beachtung der Chancengleichheit dar: Die Förderung erfolgreichen Lernverhaltens im Schulkontext sollte gewährleisten, dass Schüler(innen) mit unterschiedlichen Ausgangsvoraussetzungen

\footnotetext{
${ }^{1}$ Die Unterrichtseinheit fand im Rahmen des Faches Naturwissenschaften statt, welches die Fächer Biologie, Chemie und Physik umfasst.
} 
oder sozialen Hintergründen gleichermaßen angesprochen werden und dass nicht das Leistungsgefälle entgegen der Intention der Maßnahme vergrößert wird. In Kapitel 5 wird eine Weiterentwicklung des Fokus der Selbstregulationsforschung beschrieben: Ausgehend von der quasi-experimentellen Ausrichtung in Studie 1, die innerhalb des regulären Unterrichts im Klassenverband durchgeführt wurde, erfolgt in Studie 2 eine Hinwendung zur Untersuchung selbstregulierten Lernens auf Prozessebene, realisiert als experimentelle Laboruntersuchung. Ziel ist es, spezifische Mechanismen der Wirksamkeit instruktionalen Handelns aufzuzeigen, die die Selbstregulationsfähigkeit der Schüler(innen) beeinflussen. Die mikroanalytische Herangehensweise ist vergleichbar mit der Betrachtung des Lernprozesses durch ein Mikroskop: Mit der Untersuchung der Wirkung von Feedback und Bewertungsstandards werden zwei Ansatzpunkte exemplarisch herausgegriffen, die im Hinblick auf die Lern- und Selbstregulationsförderung optimiert werden können. Damit wird ein fokussierter Bereich von Prozessen untersucht, die aus theoretischer Sicht auch in Studie 1 wirksam sind, dort aber nicht erfasst werden. Kapitel 6 stellt die Ergebnisse der Untersuchung zum Einfluss von Feedback und Bewertungsstandards auf die Selbstregulation und den Erwerb einer neuen mathematischen Fähigkeit vor und liefert damit den zweiten Teil der Empirie zu den Forschungsfragen der Arbeit. Den beiden konzeptionell unterschiedlichen Studien liegen als verbindende Elemente zum einen die Frage nach Möglichkeiten der gleichzeitigen Förderung fachbezogener sowie selbstregulativer Fähigkeiten und zum anderen die grundlegende Annahme des zyklischen Prozesses der Selbstregulation (vgl. Kapitel 2) zugrunde. Die in der vorliegenden Arbeit gefundenen Antworten auf die Forschungsfragen (vgl. Kapitel 3), daraus abzuleitende praktische Implikationen sowie ein Ausblick für zukünftige Selbstregulationsforschung sind Bestandteile des siebten Kapitels. Es schließt mit Perspektiven für zukünftige Selbstregulationsforschung an der Schnittstelle zwischen Pädagogischer Psychologie, Entwicklungspsychologie und den Fachdidaktiken. 


\section{Selbstreguliertes Lernen aus sozial-kognitiver Perspektive}

\subsection{Die Bedeutung der sozial-kognitiven Grundannahmen für das Verständnis von Selbstregulation}

Ansätze zur Erklärung interindividueller Unterschiede in der Lern- und Leistungsfähigkeit und das daraus abgeleitete Menschenbild unterlagen in den vergangenen zwei Jahrhunderten einem starken Wandel. Im 19. Jahrhundert etwa galt Lernen als eine formale Disziplin, und Lernschwierigkeiten oder -versagen wurden in der Regel auf individuelle intellektuelle Defizite oder mangelnden Fleiß zurückgeführt. Mit Beginn des 20. Jahrhunderts erhielt die Psychologie den Stellenwert einer eigenständigen Wissenschaft und der Betrachtung interindividueller Unterschiede in leistungsbezogenen Personenmerkmalen wurde große Bedeutung beigemessen. Ausgehend von diesem Wandel vertraten Reformtheoretiker wie z.B. John Dewey (1916), Edward Lee Thorndike (1932) und Maria Montessori (Lillard, 1972; Montessori, 1964) schließlich eine neue Sicht von Erziehung im Allgemeinen und Unterricht im Speziellen. Sie forderten Modifikationen des Curriculums um den individuellen Bedürfnissen von Lernenden gerecht zu werden (Zimmerman, 2002). Die zugrunde liegende Idee, Unterrichtsinhalte zur Verbesserung des Lernerfolgs an die jeweiligen Voraussetzungen Lernender anzupassen, fand ausgehend von der sich in den sechziger und siebziger Jahren herausbildenden Instruktionspsychologie viel Beachtung. Cronbach (1957) prägte für diesen Forschungsbereich den Begriff der „Aptitude-Treatment-Interaction“. Dessen Forschungsgegenstand ist die Wechselwirkung (interaction) von individuellen Lernervoraussetzungen, also Merkmalen der lernenden Person (aptitudes), und der eingesetzten Lehrmethode (treatment) (vgl. Staemmler, 2006).

Trotz solcher wissenschaftlichen Bestrebungen, interindividuellen Unterschieden in Bedürfnissen und Möglichkeiten Rechnung zu tragen, fanden diese nur bedingt über Lehrpläne und Unterrichtsgestaltung Eingang in die Schulpraxis. Etwa in den frühen achtziger Jahren entwickelte sich durch das Zusammenlaufen von Forschungssträngen zur sozialen Lerntheorie (z.B. Rotter, 1954) und zur Metakognition eine neue Sichtweise auf Unterschiede im Lernund Leistungsvermögen junger Menschen. Der Mitbegründer und zentrale Vertreter der Theorie, Albert Bandura, erweiterte die ursprünglich als „soziale Lerntheorie“ bekannt gewordene Forschungsrichtung und änderte die Benennung in „sozial kognitive“ Theorie, um hervorzuheben, dass Kognitionen eine zentrale Rolle für die menschliche Fähigkeit spielen, Umweltereignisse zu interpretieren, Verhalten strategisch zu planen und bewusst auszuführen und Lernprozesse selbst zu regulieren (Bandura, 1977, 1986). Ein zentraler Bestandteil der Theo- 
rie ist das Konzept des reziproken Determinismus, der auf der Annahme beruht, dass a) persönliche Faktoren in Form kognitiver, emotionaler und physiologischer Aspekte, b) Verhalten und c) Umwelteinflüsse wie etwa das soziale Umfeld miteinander interagieren und reziprok Einfluss aufeinander ausüben. Die Kernaussage dieses Konzeptes formuliert Bandura (1986, S. 454) wie folgt: „Behavior is, therefore, a product of both self-generated and external sources of influence“. Vertreter(innen) der sozial-kognitiven Perspektive betrachten den Menschen als proaktiv und reflektierend anstatt als reaktives Wesen, das primär von äußeren Einflüssen gesteuert oder von inneren Impulsen getrieben wird. Individuen sind demnach sowohl aktiv Gestaltende als auch Produkte ihrer Umwelt und des sozialen Systems, in dem sie leben (Pajares, 2008).

Aus sozial-kognitiver Perspektive haben Menschen die Voraussetzungen, proaktiv Einfluss auf ihre eigene Entwicklung und auf Lernprozesse zu nehmen, diese also selbst zu regulieren. Eine Schlüsselfunktion kommt dabei der Selbstwirksamkeitserwartung zu, d.h. der Erwartung einer Person bezüglich ihrer Fähigkeit, in einer bestimmten Situation ein bestimmtes Verhalten auszuführen. Mit Banduras (1997, S. 37) Worten: „Perceived self-efficacy is concerned not with the number of skills you have, but with what you believe you can do with what you have under a variety of circumstances." Selbstwirksamkeitserwartungen sind damit kontextspezifische Überzeugungen und bilden eine zentrale Grundvoraussetzung der Motivation, der Leistungsfähigkeit und -bereitschaft von Personen.

Die Grundannahmen der sozial-kognitiven Theorie spiegeln sich auch in der Auffassung von Selbstregulation, einem Teilbereich der sozial-kognitiven Theorie, wider. Dem Konzept liegt das Prinzip des reziproken Determinismus zugrunde (vgl. Abb. 2.1): Selbstregulation stellt eine Interaktion aus personen- und verhaltensbezogenen sowie umweltbedingten Prozessen dar, die sich wechselseitig beeinflussen (Zimmerman, 2000). Eine solche Betrachtungsweise, die eine Einwirkung des handelnden Individuums auf seine Umwelt und die Rückkopplung dieser Effekte mit einbezieht, unterscheidet sich von metakognitiven Ansätzen insofern, als dass in diesen häufig in erster Linie Wissen und das Verständnis bestimmter Sachverhalte sowie die Reflexion darüber hervorgehoben werden (eine Ausnahme macht m. E. die Darstellung der Subkategorien der Metakognition von Hasselhorn, 1992, die auch bewusste kognitive Empfindungen und affektive Zustände mit einbezieht). Obwohl Metakognitionen eine zentrale Rolle spielen, hängt Selbstregulation darüber hinaus von Überzeugungen (z.B. Selbstwirksamkeitserwartungen) und affektiven Reaktionen wie Zweifel und Ängsten bezüglich bestimmter Aufgaben oder Kontexte ab (Zimmerman, 1995b). 


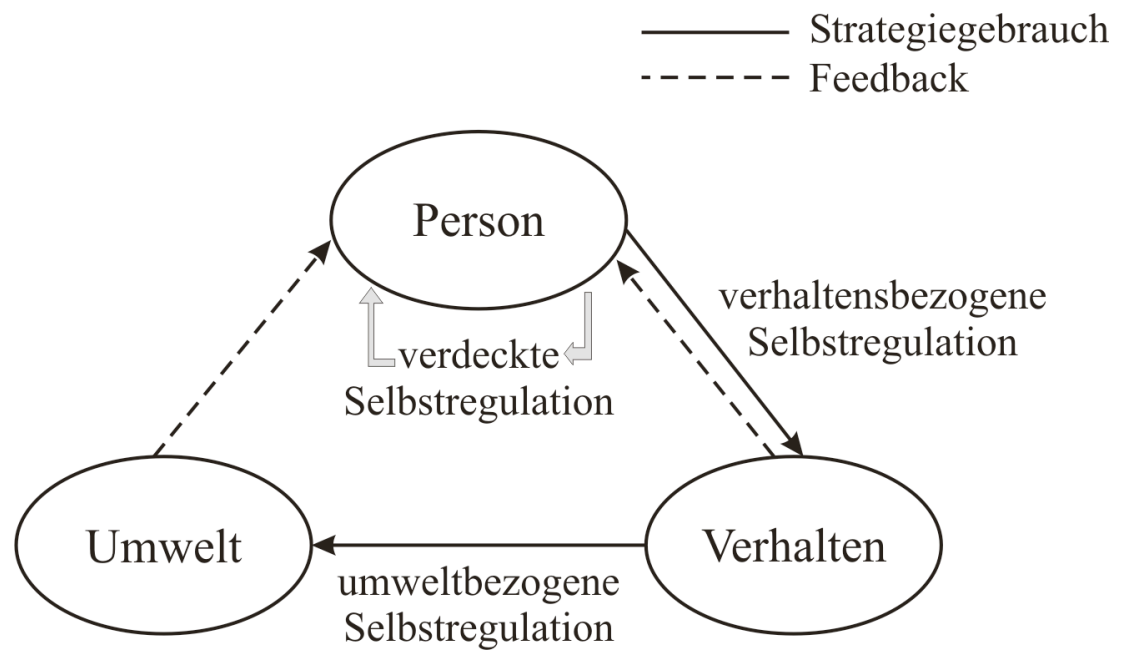

Abbildung 2.1. Wechselseitiger Einfluss der Teilbereiche der Selbstregulation nach Zimmerman (1989a)

Die Selbstregulation wird als ein zyklischer Prozess beschrieben (Zimmerman, 2000; vgl. auch Kapitel 2.1.1.), was auf die Grundannahme zurückgeht, dass Feedback, bzw. eine Rückkoppelung, ausgehend von vorherigem Verhalten, genutzt wird um Modifikationen des aktuellen und zukünftigen Verhaltens vorzunehmen. Solche Anpassungen werden nötig, da die relevanten personen- und verhaltensbezogenen sowie umweltbedingten Faktoren während der Ausführung von Aufgaben einer ständigen Veränderung und wechselseitiger Beeinflussung unterliegen. Die Beobachtung des Prozesses (,,monitoring“") erfolgt in der Modellvorstellung durch drei Rückkoppelungsschleifen, die jeweils „Ist-Soll“-Abgleiche beinhalten: Verhaltensbezogene Selbstregulation bezieht sich auf die Selbstbeobachtung und die Auswahl und Anwendung adäquater Strategien (z.B. Lernstrategien oder -methoden). Umweltbezogene Selbstregulation umfasst das Registrieren und Anpassen von Kontextfaktoren oder -einflüssen (z.B. Regulieren des Lärmpegels am Arbeitsplatz). Verdeckte Selbstregulation (,,covert selfregulation“, Zimmerman, 2000) bezieht sich auf die Beobachtung und die Modifikation kognitiver und affektiver Zustände (z.B. das Wahrnehmen von Stress und die Suche nach Entspannung als Reaktion darauf). Diese drei Rückkoppelungsschleifen werden als offene Feedback-Systeme betrachtet. Im Gegensatz zu Ansätzen, die von geschlossenen Rückkoppelungssystemen ausgehen, in denen Selbstregulation darauf beschränkt ist, dass reaktiv Diskrepanzen zwischen einem Ist-Zustand und einem unveränderlichen Standard (Soll-Zustand) reduziert werden, bezieht der sozial-kognitive Ansatz mit der Annahme offener Rückkoppelungsschleifen darüber hinaus mit ein, dass Diskrepanzen auch temporär proaktiv vergrößert 
werden können. Dies ist etwa durch eine Erhöhung der persönlichen Zielvorstellungen oder die Wahl stärker herausfordernder Aufgaben möglich (Zimmerman, 2000). Solche selbsterzeugten Diskrepanzen dienen Personen als Quelle von Motivation ihre Fähigkeiten zu erweitern und sich persönlich zu entwickeln. Grundsätzlich hängt die Art und Weise, wie Lernende mit negativen Diskrepanzen (der Bewertungsstandard übersteigt die tatsächliche Leistung) umgehen, entsprechend der Grundgedanken der Theorie von kontext- und personenspezifischen Faktoren ab: Manche Personen entwickeln als Reaktion bessere Strategien und intensivieren ihre Anstrengung um internen Standards zu entsprechen, andere reduzieren die Höhe dieser Standards und wieder andere halten an eigenen hohen Ansprüchen fest, verlieren aber ihre Motivation angesichts steigender Mutlosigkeit bei Misserfolg. Ausschlaggebend für die Art und Richtung der Reaktion ist die kontextspezifische Selbstwirksamkeitserwartung einer Person, d.h. ihre Überzeugung bezüglich ihrer Fähigkeit eine bestimmte Aufgabe letztendlich erfolgreich zu bewältigen.

Zusammenfassend lässt sich für die Bedeutung der sozial-kognitiven Theorie für das Verständnis von Selbstregulation festhalten, dass grundsätzlich von wechselseitig interagierenden Faktoren (Person, Verhalten und Umwelt) ausgegangen wird, auf welche das Individuum sowohl proaktiv als auch reaktiv im Hinblick auf das Erreichen persönlicher Ziele bzw. angestrebter Zustände Einfluss nimmt. Für die Betrachtung der Selbstregulation in Bezug auf den Erwerb von Fähigkeiten und Fertigkeiten, also des selbstregulierten Lernens, bedeutet die hier skizzierte Perspektive, dass es sich dabei nicht um eine statische mentale Fähigkeit oder akademische Leistungskomponente im Sinne einer Persönlichkeitseigenschaft (trait), sondern um einen kontextspezifischen, selbstgesteuerten Prozess handelt, in dessen Verlauf Lernende ihre mentalen Fähigkeiten in (schul-)leistungsbezogene Fertigkeiten transferieren. Dieser Prozess beinhaltet metakognitive, kognitive, motivationale und verhaltensbezogene Komponenten. Lernen wird gemäß dieser Auffassung als eine Aktivität betrachtet, die in erster Linie proaktiv von Lernenden ausgeht, anstatt als ein Ereignis, das als Reaktion auf Unterricht in Schüler(inne)n verursacht wird (Zimmerman, 2002). 


\subsection{Die Theorie selbstregulierten Lernens nach Zimmerman}

\subsubsection{Selbstreguliertes Lernen als zyklischer Prozess}

Die Selbstregulationstheorie nach Zimmerman (2000) basiert auf der sozial-kognitiven Theorie und knüpft an die Grundgedanken dieser Forschungsrichtung an. Zentral ist die oben bereits erwähnte Auffassung, dass Selbstregulation keine stabile Persönlichkeitseigenschaft darstellt, die genetisch verankert oder erworben ist, sondern durch zyklisch verlaufende, kontextspezifische (Sub-)Prozesse definiert ist (vgl. Hasselhorn \& Labuhn, 2008). Diese Prozesse beinhalten über metakognitive Fähigkeiten hinaus auch affektive und verhaltensbezogene Komponenten. Die wechselseitige Abhängigkeit der Prozesse wird von Zimmerman (2000) im Rahmen dreier zyklischer Phasen beschrieben: Vorbereitungsphase, Handlungsphase und Selbstreflexionsphase (vgl. Abb. 2.2). Die Vorbereitungsphase bezieht sich auf jene Prozesse, die der eigentlichen Lernhandlung vorausgehen und die Voraussetzungen dafür schaffen. In der Handlungsphase laufen Prozesse ab, die sich direkt auf das aktuelle Lernen oder die Bearbeitung von Aufgaben beziehen. Die Selbstreflexionsphase beinhaltet Prozesse der Reflexion und Bewertung der abgeschlossenen Handlung. Das Ergebnis der Selbstreflexion beeinflusst wiederum die nachfolgende Vorbereitungsphase und damit zukünftiges Lernen. Die Inhalte und Funktionen der einzelnen Phasen nach Zimmerman $(2000,2002)$ werden im Folgenden zusammenfassend dargestellt.

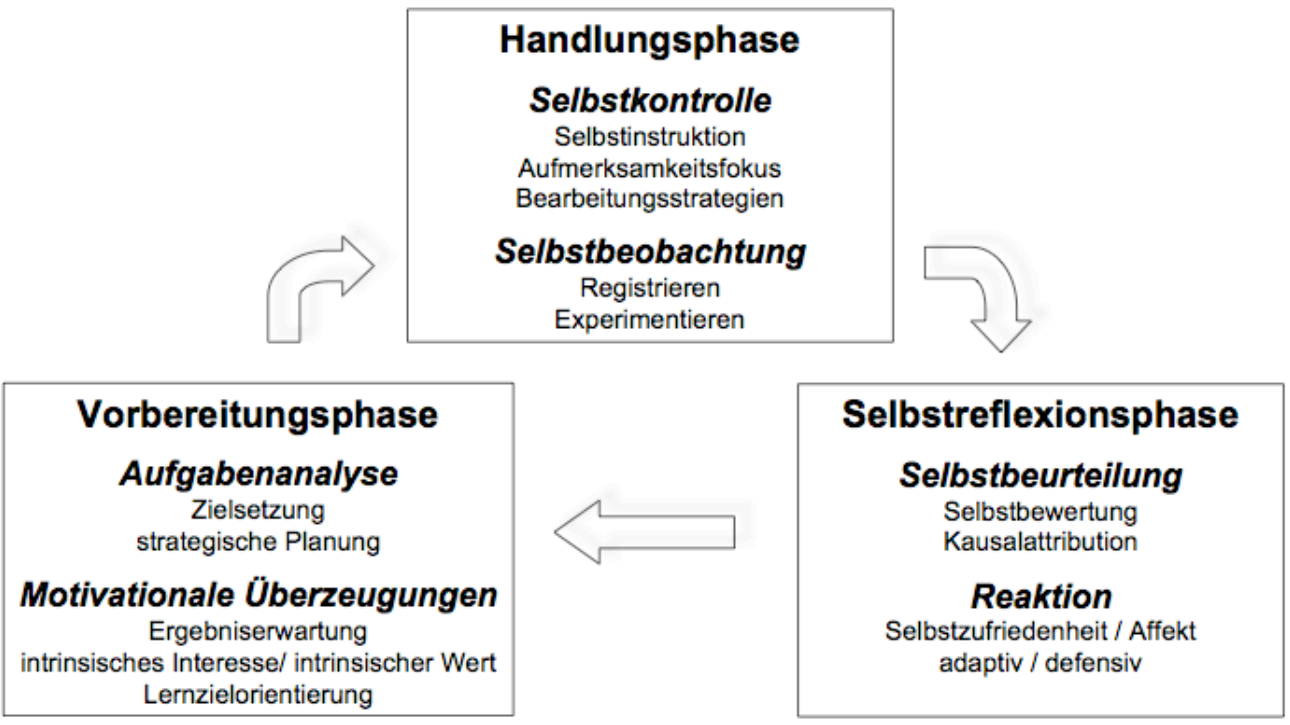

Abbildung 2.2. Phasenmodell des selbstregulierten Lernens nach Zimmerman und Campillo (2003) in der deutschen Übersetzung von Hasselhorn und Labuhn (2008) 


\section{Vorbereitungsphase}

Die Prozesse dieser Phase lassen sich zwei distinkten, jedoch eng miteinander in Verbindung stehenden Kategorien zuordnen: a) Aufgabenanalyse und b) motivationale Überzeugungen. Ein zentraler Aspekt der Aufgabenanalyse stellt die persönliche Zielsetzung in Bezug auf Lernergebnisse oder die Aufgabenbearbeitung dar. Das System persönlicher Ziele ist bei Personen mit ausgeprägter Selbstregulationsfähigkeit hierarchisch geordnet, so dass prozessorientierte Ziele als proximale Regulatoren für distale, z.B. leistungsbezogene Ziele fungieren. Schüler(innen) können sich z.B. das Ziel setzen, regelmäßig ihre Mathematikhausaufgaben zu erledigen (proximales prozessorientiertes Ziel), um ihre Mathematiknote am Ende des Schuljahres zu verbessern (distales leistungsbezogenes Ziel). Ein zweiter Aspekt der Aufgabenanalyse ist die strategische Planung. Um ein Lernziel zu erreichen oder eine Aufgabe erfolgreich auszuführen, benötigen Lernende angemessene Strategien. Auswahl und Anwendung geeigneter Strategien müssen gegebenenfalls während des Lernens und Arbeitens modifiziert werden, da personen- und verhaltensbezogene Prozesse sowie Kontextfaktoren ständigen Veränderungen unterliegen. Das bedeutet, dass keine Strategie für alle Personen gleich hilfreich ist. Selbst für ein und dieselbe Person ist es unwahrscheinlich, dass sich eine Strategie universell für alle Arten von Aufgaben, Kontexten und Fähigkeitsausprägungen eignet. Selbstregulationsfähigkeiten wie Zielsetzung und strategische Planung sind von geringem Wert, wenn Lernende nicht motiviert sind, sie auch anzuwenden. Den Prozessen der Vorbereitungsphase unterliegen eine Reihe motivationaler Überzeugungen, welche die Richtung des Verhaltens erheblich beeinflussen. Dazu gehören z.B. Selbstwirksamkeitserwartungen, das Interesse oder der Wert, der einer bestimmten Aufgabe beigemessen wird, und die Zielorientierung. Diese Überzeugungen wirken sich auf die Prozesse der Aufgabenanalyse aus. So hat z.B. die Selbstwirksamkeitserwartung, mit der eine Person an eine bestimmte Aufgabe herangeht, Konsequenzen für die persönliche Zielsetzung: Je fähiger sich Personen bezüglich der Bewältigung bestimmter Aufgaben einschätzen, desto höhere Ziele setzen sie sich und desto konsequenter verfolgen sie diese auch (Bandura, 1991; Locke \& Latham, 1990). Die gesetzten Ziele können im Gegenzug auch Effekte auf die Selbstwirksamkeitserwartungen haben: Empirische Belege zeigen, dass das Erreichen prozessorientierter Ziele als solches motivationsfördernd wirkt und sogar eine günstigere Wirkung haben kann als das Erreichen leistungsorientierter Ziele (Schunk \& Schwartz, 1993; Zimmerman \& Kitsantas, 1997). Sowohl Zielsetzung und strategische Planung, als auch motivationale Überzeugungen nehmen 
einen direkten Einfluss auf den weiteren Verlauf des Selbstregulationszyklus, das heißt, auf die Prozesse der Handlungsphase.

\section{Handlungsphase}

Die zwei Hauptkategorien dieser Phase sind Selbstkontrolle und Selbstbeobachtung. Selbstkontrollprozesse wie Selbstinstruktion, die bildliche Vorstellung von Sachverhalten, Fokussierung der Aufmerksamkeit und Lern- bzw. Bearbeitungsstrategien unterstützen die Konzentration auf die Aufgabe und optimieren deren Ausführung. Selbstinstruktion bezieht sich auf die offene oder verdeckte Verbalisierung des Lösungsprozesses bzw. die Beschreibung des Vorgehens. Forschungsergebnisse zur Selbstinstruktion belegen, dass dadurch das Lernen und die Leistung unterstützt wird (Schunk, 1982; Meichenbaum, 1977). Die Fokussierung der Aufmerksamkeit als eine weitere Form der Selbstkontrolle unterstützt die Konzentration auf eine bestimmte Aufgabe und das Ausblenden ablenkender Einflüsse. Kuhl (1985) zeigt, dass die Anwendung volitionaler Kontrollstrategien wie das Ignorieren von Störungen und Ablenkungen den Bearbeitungsprozess effektiv positiv beeinflusst. Die zentrale Rolle der Anwendung adäquater Lern- oder Bearbeitungsstrategien wurde an vielen Stellen belegt (z.B. Weinstein \& Mayer, 1986; Wood, Woloshyn \& Willoughby, 1995; Zimmerman \& MartinezPons, 1986; 1988, 1990). Dazu gehören z.B. die Visualisierung von Lerninhalten, Techniken des Notierens und Unterstreichens, die strukturierte Vorbereitung auf Tests und Klausuren, verständnisorientiertes Lesen und Strategien zum Einprägen von Fakten.

Die zweite Hauptkategorie der Prozesse der Handlungsphase stellt die Selbstbeobachtung dar. Diese bezieht sich auf die Beobachtung und das Verfolgen von Arbeitsweise und -bedingungen (self-monitoring) sowie der erzielten Resultate. Die Effektivität der Selbstbeobachtung kann von verschiedenen Aspekten beeinflusst werden. Der Informationsgehalt von Feedback wirkt sich zum Beispiel auf die Qualität der Beobachtung des eigenen Arbeitsprozesses und der Leistung aus (Schunk \& Rice, 1993; Schunk \& Schwartz, 1993; Zimmerman, 1989b, 1995a). Informative Rückmeldungen unterstützen die Genauigkeit der Selbstbeobachtung, die ein wichtiges Qualitätsmerkmal darstellt: Personen, die ihre Handlungen nicht richtig einschätzen oder verzerrt wahrnehmen, können sie auch nicht adäquat korrigieren. Das Registrieren und Protokollieren eigener Leistungen (self-recording) ist eine gängige Selbstbeobachtungstechnik und erhöht zusätzlich den Informationsgehalt, die Genauigkeit und die Effektivität von Feedback (Kitsantas \& Zimmerman, 2006; Zimmerman \& Kitsantas, 1996). Die Selbstbeobachtung kann eine Person zudem dazu anregen, mit ihrem Verhalten zu experimentieren (self-experimentation; Bandura, 1991), indem sie systematisch die fraglichen As- 
pekte des eigenen Verhaltens variiert. Beispiele für kleine Verhaltensexperimente sind etwa das Anwenden verschiedener Lernstrategien, das Lernen zu unterschiedlichen Uhrzeiten oder an verschiedenen Orten (Bibliothek, eigener Schreibtisch, Wohnzimmer). Auf diese Weise kann systematische Selbstbeobachtung zu einem besseren Verständnis für eigene Voraussetzungen und Bedürfnisse führen und im günstigen Fall die Leistung positiv beeinflussen.

\section{Selbstreflexionsphase}

Die zwei Kategorien der Selbstreflexionsprozesse - Selbstbeurteilung und Selbsteinschätzung - stehen in enger Verbindung mit der Selbstbeobachtung. Im Gegensatz zu dieser laufen sie jedoch erst im Anschluss an die Phase des Lernens bzw. der Aufgabenbearbeitung ab. Selbstbeurteilung beinhaltet die Bewertung der eigenen Leistung und die kausale Attribution der Ergebnisse auf zugrunde liegende Ursachen. Selbsteinschätzung bezieht sich auf einen Vergleich der beobachteten Ergebnisse mit einem Standard oder in der Vorbereitungsphase gesetzten Ziel. Die Genauigkeit der Selbsteinschätzung stellt einen sehr entscheidenden Faktor im Selbstregulationszyklus dar, da sie die Grundlage für Adaptationen der den Lernund Arbeitsprozess beeinflussenden Komponenten bildet: Nur auf der Basis einer realistischen und genauen Einschätzung der eigenen Leistung können adäquate Modifikationen (z.B. von Lernstrategien und Arbeitsweise) im Hinblick auf die Zielerreichung gemacht werden. Die Bewertung der eigenen Leistung steht in enger Verbindung mit der kausalen Attribution der Resultate, z.B. der Rückführung von Misserfolg auf mangelnde Fähigkeiten oder unzureichende Anstrengung. Attributionen spielen eine Schlüsselrolle im Selbstreflexionsprozess: Führen Personen Fehler oder Misserfolg auf ihre geringe Fähigkeit zurück und nehmen sie an, diese sei ein stabiles Merkmal (,ich war schon immer schlecht in Mathe“), wirkt sich dies negativ auf ihre Motivation und die zukünftige Auseinandersetzung mit entsprechenden Aufgaben aus (Weiner, 1979). Im Gegensatz hierzu kann die Attribution von Fehlern auf lediglich inadäquate Lernstrategien zur Aufrechterhaltung von Motivation und Anstrengung trotz suboptimaler Leistung führen: Strategien werden als veränderbar wahrgenommen und die Aussicht auf Erfolg bei Anwendung adäquater Methoden bleibt bestehen (z.B. Zimmerman \& Kitsantas, 1996, 1997). Selbstbewertung und Selbsteinschätzung rufen zwei Kategorien möglicher Reaktionen hervor: Zufriedenheit bzw. Unzufriedenheit und adaptive bzw. defensive Schlussfolgerungen. Das Empfinden von Zufriedenheit oder Unzufriedenheit mit der eigenen Leistung und deren Einschätzung hat Konsequenzen für Motivation und Selbstwirksamkeitserwartungen bezüglich zukünftiger Aufgaben. Die adaptiven (oder defensiven) Schlussfolgerungen einer Person beziehen sich auf mögliche Modifikationen (bzw. starres Beibehalten) 
der Herangehensweise für nachfolgende Lernhandlungen oder die Bearbeitung von Aufgaben. Damit nehmen die Prozesse der Selbstreflexionsphase - entsprechend der Modellvorstellung - entscheidenden Einfluss auf den weiteren Verlauf des Selbstregulationszyklus: Sie wirken sich auf die darauf folgende Vorbereitungsphase sowohl auf motivationaler Ebene (z.B. auf die Selbstwirksamkeitserwartungen) als auch auf kognitiver und verhaltensbezogener Ebene aus (z.B. durch Adaptation von Strategien oder Modifikation von Zielen). Zudem können sie die nächste Handlungsphase, z.B. auf metakognitiver Ebene durch exaktere Selbstbeobachtung (self-monitoring), beeinflussen.

\subsubsection{Vier Entwicklungsniveaus der Selbstregulation}

Zimmerman (2000) macht im Rahmen seiner Theorie nicht nur Annahmen über den Prozess der Selbstregulation beim Lernen oder Bearbeiten von Aufgaben (dargestellt in Kapitel 2.2.1.), sondern auch über die Entwicklung der Selbstregulationsfähigkeit. Entsprechend der sozial-kognitiven Perspektive erfolgt der Erwerb neuer Fähigkeiten und Fertigkeiten über vier Entwicklungsniveaus hinweg und wird zunehmend stärker selbstreguliert (Zimmerman, 2000; Zimmerman \& Kitsantas 1997, 1999) (vgl. Tab. 2.1). Diese Niveaus repräsentieren die steigende metakognitive, motivationale und verhaltensbezogene Regulation einer bereichsspezifischen Fertigkeit und zunehmende Unabhängigkeit von sozialen Modellen bei der Ausführung des Verhaltens. Es wird angenommen, dass Lernende, die die Niveaus sequentiell bewältigen, Fähigkeiten und Fertigkeiten leichter und effektiver erwerben. Zimmerman (2000) geht jedoch nicht davon aus, dass Personen, die das vierte und höchste Niveau erreicht haben, das damit verbundene Verhalten auch konsequent zeigen. Stattdessen betrachtet er die Fähigkeitsausprägung als kontextspezifisch. In Abgrenzung zu entwicklungspsychologischen Stufenmodellen (z.B. Kohlberg, 1981; Piaget, 1962; Selman, 1980) impliziert die Formulierung der vier Stufen keine invariante Abfolge der Entwicklungsniveaus. 
Tabelle 2.1. Entwicklungsniveaus der Selbstregulationsfähigkeit nach Zimmerman

\begin{tabular}{|c|c|c|c|}
\hline Niveau & Beschreibung & Soziale Einflüsse & $\begin{array}{l}\text { Personenbezogene Ein- } \\
\text { flüsse }\end{array}$ \\
\hline 1. Beobachtung & $\begin{array}{l}\text { Stellvertretender Erwerb einer } \\
\text { Fertigkeit durch Beobachtung } \\
\text { eines erfahrenen Verhaltensmo- } \\
\text { dells }\end{array}$ & $\begin{array}{l}\text { Modeling } \\
\text { Erklären (verbal) }\end{array}$ & \\
\hline 2. Nachahmung & $\begin{array}{l}\text { Nachahmende Ausführung des } \\
\text { grundlegenden Musters des } \\
\text { Modellverhaltens mit sozialer } \\
\text { Anleitung }\end{array}$ & $\begin{array}{l}\text { Anleitung } \\
\text { Feedback }\end{array}$ & \\
\hline 3. Selbstkontrolle & $\begin{array}{l}\text { Eigenständige Ausführung des } \\
\text { Modellverhaltens unter struktu- } \\
\text { rierten Bedingungen }\end{array}$ & $\begin{array}{l}\text { Lediglich interne Reprä- } \\
\text { sentation des Verhaltens } \\
\text { anderer Personen (Ver- } \\
\text { haltensmodelle) }\end{array}$ & $\begin{array}{l}\text { Internale Standards } \\
\text { Selbstverstärkung } \\
\text { Monitoring }\end{array}$ \\
\hline 4. Selbstregulation & $\begin{array}{l}\text { Adaptiver Einsatz der Fertigkeit } \\
\text { über verschiedene personen- } \\
\text { und umweltbezogene Bedin- } \\
\text { gungen hinweg }\end{array}$ & & $\begin{array}{l}\text { Selbstregulationsprozesse } \\
\text { Motivation } \\
\text { (z.B. Selbstwirksamkeits- } \\
\text { überzeugung) }\end{array}$ \\
\hline
\end{tabular}

Auf dem ersten Niveau der Selbstregulation, Beobachtung (observation), erwerben Lernende durch die Beobachtung eines Verhaltensmodells, d.h. einer anderen Person, die über Expertise auf dem entsprechenden Gebiet verfügt, eine Vorstellung der zentralen Aspekte einer Fertigkeit oder Strategie. Über das zu erwerbende Verhalten hinaus übermitteln Modelle auch zusätzliche selbstregulative Prozesse, z.B. Bewertungsstandards, motivationale Orientierungen und Werte. Den stellvertretenden Erfahrungen mit der Ausführung des angestrebten Verhaltens kommt somit ein hoher Stellenwert zu, jedoch müssen Personen entsprechende Strategien oder Abläufe in der Regel erst selbst in die Tat umsetzen, um sie in ihr persönliches Verhaltensrepertoire aufzunehmen. Das Niveau der Nachahmung (emulation) ist erreicht, wenn Lernende in der Lage sind, das Zielverhalten so auszuführen, dass es dem Verhalten des Modells ungefähr entspricht. Meistens wird nicht exaktes Verhalten kopiert, sondern das zugrunde liegende Muster oder der Stil der Ausführung wird nachgeahmt. Nimmt das Verhaltensmodell eine Lehrer(innen)- oder Trainer(innen)rolle ein und stellt es Anleitung, Feedback und Verstärkung des Zielverhaltens im Lernprozess bereit, kann dies zudem die Genauigkeit der Nachahmung unterstützen. Während die wichtigste Informationsquelle für die zu erwerbende Fertigkeit auf den ersten zwei Entwicklungsniveaus noch schwerpunktmäBig das soziale Umfeld ist, verschiebt sich der Fokus auf den weiter fortgeschrittenen Niveaus in Richtung der Prozesse, die innerhalb des lernenden Individuums ablaufen. Der Erwerb ei- 
ner Fertigkeit erfordert in der Regel mehr als lediglich die Beobachtung und Nachahmung des Zielverhaltens: Für viele Bereiche gilt, dass höhere Selbstregulationsfähigkeiten erst durch bewusstes und beharrliches eigenständiges Üben zu erlangen sind (Ericsson \& Lehman, 1996). Das dritte Niveau der Selbstregulation, die Selbstkontrolle (self-control), ist erreicht, wenn Personen eine entsprechende Strategie oder einen Verhaltensablauf planen, eigenständig ausführen und ihren eigenen Prozess beobachten (self-monitoring). Obwohl Lernende auf diesem Niveau nicht mehr auf die Anwesenheit eines Verhaltensmodells angewiesen sind, bleiben sie noch abhängig von der mentalen Repräsentation des Modells und dessen Leistungsstandards. Die Anwendung aufgabenbezogener Strategien, das Setzen prozessorientierter Ziele und die genaue Selbstbeobachtung fördern die Entwicklung der Automatisierung des Verhaltens, die eine zentrale Voraussetzung für das vierte und höchste Niveau, die Selbstregulation (self-regulation) darstellt. Ist dieses Niveau erreicht, sind Lernende in der Lage, ihr Verhalten systematisch an sich verändernde persönliche und kontextuelle Bedingungen anzupassen. Sie wählen aus einem Repertoire geeignete Strategien aus, wenden diese an und modifizieren ihre Herangehensweise basierend auf ihrer Beobachtung der erzielten Ergebnisse. Obwohl Personen auf diesem Niveau die Fähigkeit besitzen, Verhalten selbstreguliert auszuführen, nutzen sie diese nicht notwendigerweise: Die gewissenhafte Planung, Konzentration und ein gewisses Maß an Anstrengung sind mental und physisch anspruchsvoll. Wichtige Voraussetzungen der Bereitschaft die notwendige Energie zu investieren sind das Interesse an der Bewältigung einer bestimmten Aufgabe sowie die Selbstwirksamkeitsüberzeugung einer Person hinsichtlich der erfolgreichen Bewältigung dieser Aufgabe. Kitsantas, Zimmerman und Cleary (2000), sowie Zimmerman und Kitsantas (1996, 1997, 1999) validierten das Konzept der Entwicklungsniveaus in verschiedenen Studien zum Erwerb motorischer Fähigkeiten und Strategien zur Revision geschriebener Texte.

Zusammenfassend ist festzuhalten, dass die durch vier Niveaus beschriebene Entwicklung der Selbstregulationsfähigkeit mit der Beobachtung eines Verhaltensmodells und ausführlicher sozialer Anleitung beginnt, die zum Erwerb des Wissens über die Ausführung des selbstregulativen Zielverhaltens (z.B. eine Aufgabenstrategie) führt (Beobachtung), welches dann in tatsächliches Verhalten umgesetzt wird (Nachahmung). Die Abhängigkeit von der externen Unterstützung wird mit zunehmender Internalisierung des Verhaltens (Selbstkontrolle) systematisch reduziert, bis Lernende in der Lage sind, entsprechende Fertigkeiten oder Strategien automatisiert und selbstreguliert auszuführen (Selbstregulation). Es handelt sich dabei grundsätzlich um einen kontextspezifischen Prozess, der sich auf einen bestimmten Inhaltsbereich bezieht. Die Niveaustufen unterscheiden sich hinsichtlich des Ausmaßes, in dem 
der oder die Lernende von der Hilfe eines sozialen Modells abhängig ist und wie sehr er oder sie in der Lage ist, lernstrategisches Wissen und Können auf unterschiedliche Aufgaben und Kontexte anzuwenden. ${ }^{2}$

\footnotetext{
2 Einen verwandten Ansatz mit anderem Fokus stellt Hasselhorn (1996) dar. Im Mittelpunkt seines Konzepts stehen Defizite der lernenden Person, die einer zielführenden und flexiblen Anwendung von Lernstrategien entgegenstehen. Im Laufe der Entwicklung lernstrategischer Kompetenz werden diese Defizite überwunden, was bei erfolgreichem Verlauf zum Erreichen der höchsten Stufe, der Strategiereife, führt. Auf dieser Stufe werden Lernstrategien zeitlich stabil und über verschiedene Kontexte hinweg angewandt. Zimmerman (2000) und Hasselhorn (1996) stellen damit den Erwerb selbstregulationsbezogener bzw. strategischer Fertigkeiten übereinstimmend als Abfolge von Stufen bzw. Niveaus dar, die einen kontinuierlichen Entwicklungsprozess beschreiben und die in der Beschreibung des höchsten Niveaus - der Selbstregulation (Zimmerman) bzw. Strategiereife (Hasselhorn) - maximale Ähnlichkeit aufweisen.
} 


\section{Selbstregulationsförderung unter sozial-kognitiven Gesichtspunkten}

\subsection{Implikationen der sozial-kognitiven Theorie für schulisches Lernen}

Es liegen zahlreiche Forschungsergebnisse vor, die einen engen Zusammenhang zwischen selbstregulativen Prozessen und Schulleistung belegen (z.B. Schunk, 1994; Zimmerman \& Bandura, 1994; Zimmerman \& Martinez-Pons, 1986, 1988, 1990). Aufgrund der motivationalen und (meta-) kognitiven Vorteile des selbstregulierten Fertigkeits- und Fähigkeitserwerbs lassen Interventionsansätze zur Förderung der Selbstregulation gleichzeitig auch positive Effekte auf schulische Leistungen erwarten. In diesem Kontext stellt Pajares (2008, p. 112) fest ,Social cognitive theory provides multiple lenses through which to view the manner in which appropriate actions can be undertaken to improve human functioning". Der Darstellung der theoretischen Fundierung selbstregulierten Lernens schließt sich damit die Frage nach den Implikationen der sozial-kognitiven Perspektive für die pädagogisch-psychologische Praxis, insbesondere für die Gestaltung von Lehr-Lern-Situationen, an: Wie kann die Entwicklung der Selbstregulationsfähigkeit von Lernenden unterstützt und optimiert werden und welche Anhaltspunkte liefern die Grundgedanken der sozial-kognitiven Theorie für die Konzeption von Interventionen? Im vorliegenden Kapitel werden in einem ersten Schritt, ausgehend von den elementaren sozial-kognitiven Prinzipien und dem daraus hervorgehenden Verständnis selbstregulierten Lernens (vgl. Kapitel 2.1 und 2.2), Implikationen für die Förderung selbstregulierten Lernens im Schulkontext abgeleitet. Dabei wird auf das Konzept des reziproken Determinismus, die Formulierung von Entwicklungsniveaus der Selbstregulationsfähigkeit, die Annahme proaktiv Lernender und die Auffassung von selbstreguliertem Lernen als einem zyklischen Prozess eingegangen. In einem zweiten Schritt werden einige bereits evaluierte Ansätze zur Förderung selbstregulierten Lernens im Schulkontext und insbesondere im regulären Unterricht vorgestellt, die sich in ihrer theoretischen Fundierung auf die sozialkognitive Auffassung von Selbstregulation (Zimmerman, 2000) beziehen (Kapitel 3.2). In Kapitel 3.3 werden anknüpfend an Desiderate der Selbstregulationsförderung die Forschungsfragen des empirischen Teils dieser Arbeit aufgeworfen.

Wie in Kapitel 2.1 dargestellt, bildet das Konzept des reziproken Determinismus einen zentralen Bestandteil der sozial-kognitiven Theorie. Der Erwerb von Fertigkeiten und Fähigkeiten, insbesondere das schulische Lernen, geschieht nicht isoliert als eine bloße Reaktion auf Unterricht, sondern innerhalb eines komplexen Netzwerkes sozialer Interaktion und weiterer Kontextfaktoren, die untereinander und mit dem Individuum interagieren. Im Schulkon- 
text bedeutet dies zum Beispiel: Lehrkräfte können ihre Schüler(innen) unterstützen, indem sie deren emotionale Verfassung, Motivation, Überzeugungen über eigene Fähigkeiten oder Denkweisen (personenbezogene Faktoren) günstig beeinflussen und schulleistungsbezogene sowie selbstregulative Fähigkeiten und Fertigkeiten fördern (Verhalten). Sie können darüber hinaus die soziale Struktur im Klassenraum, die Arbeitsatmosphäre und Rahmenbedingungen (umweltbezogene Faktoren) mit einbeziehen und gegebenenfalls modifizieren. Dabei gilt entsprechend der reziproken Beziehung der Einflussfaktoren, dass Veränderungen in einem der Bereiche Veränderungen in den anderen bewirken können (Pajares, 2008).

Zur zentralen Funktion sozialer Einflüsse innerhalb der sozial-kognitiven Theorie (im Konzept des reziproken Determinismus den „umweltbezogenen Faktoren“ zugeordnet) zählt auch das stellvertretende Lernen (oder auch Modelllernen, vgl. Bandura, 1969), d.h. der Erwerb von Fertigkeiten, welcher von der Beobachtung und Nachahmung anderer ausgeht. Verhaltensmodelle, die über eine gewisse Erfahrung bei der Ausführung eines bestimmten Verhaltens oder der Anwendung einer Strategie verfügen, spielen damit eine wichtige Rolle im Lernprozess. Dies spiegelt sich auch in der Formulierung der Entwicklungsniveaus der Selbstregulationsfähigkeit (Zimmerman, 2000) wider. Für die Gestaltung schulischer Lernsituationen und die Vermittlung von Selbstregulationsfähigkeiten bedeutet dies, Schüler(inne)n besonders zu Beginn des Lernprozesses genaue Anleitung und nach Möglichkeit Verhaltensmodelle anzubieten: Nur wenn Lernenden eine Vorstellung davon vermittelt wird, wie das Zielverhalten aussieht und wie sie es erwerben können, werden sie auch in der Lage sein, dieses schließlich selbstreguliert auszuführen. Das gilt sowohl für fachbezogene Lerninhalte als auch besonders für die Vermittlung von Selbstregulationskomponenten. Aspekte wie die Zielsetzung vor dem Lernen, die Anwendung adäquater Lern- und Bearbeitungsstrategien sowie eine gewissenhafte Beobachtung des eigenen Lernprozesses (self-monitoring) existieren im Verhaltensrepertoire der meisten Schüler(innen) zunächst nicht und erscheinen ihnen infolgedessen ungewohnter als manche fachbezogenen Fähigkeiten. Dies unterstreicht die Notwendigkeit einer anfänglichen expliziten Anleitung. Zudem ist die Interaktion der Schüler(innen) untereinander von Bedeutung, die sich mit zunehmender Sicherheit bei der Ausführung des Verhaltens auch gegenseitig als Modelle dienen können.

In diesem Stadium der Fähigkeitsentwicklung spielt externes Feedback eine wichtige Rolle. Es stellt Informationen über den Stand des Lernprozesses bereit, erfüllt die Funktion, die Ausführung des Zielverhaltens zu verstärken und unterstützt die Genauigkeit von Selbstreflexion und -einschätzung. Die Rückmeldung ermöglicht es Lernenden, zielführende Modifikationen ihres Verhaltens vorzunehmen. Ist die anfängliche Anleitung und enge Betreuung 
im Lernprozess noch nötig und sinnvoll, so ist es ebenfalls wichtig, diese im Laufe der Fähigkeitsentwicklung zu reduzieren und Lernenden die Möglichkeit zu gewähren, die Kontrolle und Steuerung selbst zu übernehmen, sich auf internale Standards zu beziehen und sich auf internes Feedback zu verlassen. Das bedeutet, Schüler(inne)n die Möglichkeiten und den Freiraum bereitzustellen, fachbezogene sowie selbstregulative Fähigkeiten eigenständig auszuführen und in mehrfacher Auseinandersetzung mit entsprechenden Aufgaben zu üben.

Die Förderung von Selbstregulation setzt jedoch nicht zwangsläufig, wie manchmal irrtümlich angenommen, voraus, dass Aufgaben gewisse Merkmale aufweisen (z.B. offene Fragestellungen mit viel Gestaltungsspielraum) oder Unterrichtsmethoden und Arbeitsform bestimmten Kriterien entsprechen müssen (z.B. Gruppen- und Freiarbeit oder kooperatives Lernen). Sicher kann die Selbstregulation der Schüler(innen) durch eine entsprechend freie Aufgabenstellung und Gestaltung der Lernsituation begünstigt werden, grundsätzlich ist sie jedoch, wie in Kapitel 2 bereits ausführlich dargestellt, auf einer anderen als der strukturellen Ebene angesiedelt: Da es sich bei selbstreguliertem Lernen nicht um eine didaktische Methode, sondern um den individuellen und kontextspezifischen Prozess der reflektierten Aneignung und Optimierung einer Fähigkeit oder Fertigkeit handelt, kann es - ein gewisses Maß an Handlungsspielraum und Übungsmöglichkeiten vorausgesetzt - durchaus in regulären Unterrichts- und außerschulischen Lernsituationen stattfinden. Das Prinzip der allmählichen Reduktion der externen Verhaltenskontrolle und Förderung der selbstregulierten Ausführung des Zielverhaltens sollte in die Vermittlung von sowohl fachbezogenen Inhalten als auch selbstreguliertem Lernen einfließen.

Die Formulierung der vier Entwicklungsniveaus impliziert weiterhin, dass Lernende darin unterstützt werden sollten, ein einmal erreichtes, viertes und höchstes Niveau der Selbstregulation aufrechtzuerhalten. Einen entscheidenden Faktor für die weitere Anwendung der elaborierten selbstregulativen Fähigkeiten stellt die Motivation einer Person dar, das entsprechende Verhalten auch tatsächlich zu zeigen. Wie oben erläutert (Kapitel 2.2.2), kann nicht angenommen werden, dass dies unter allen Umständen der Fall ist. In der Praxis spielt besonders ein Aspekt für die motivationalen Voraussetzungen eine wichtige Rolle: Lernende müssen selbst den Nutzen der Anwendung entsprechenden Verhaltens, z.B. des Einsatzes einer Strategie, feststellen. Führt sie die eigene positive Erfahrung zu der Einsicht, dass sich die Investition von Anstrengung und Konzentration lohnt und sie davon z.B. im Hinblick auf schulische Leistungen profitieren, ist die Aufrechterhaltung und Anwendung ihrer Selbstregulationsfähigkeit wahrscheinlicher. Im Rahmen von Ansätzen zur Förderung selbstregulierten Lernens sollten Schüler(innen) also wiederholt die Möglichkeit erhalten, derartige Erfolgser- 
lebnisse zu verzeichnen, was erneut die Relevanz ausreichender Übungsmöglichkeiten unterstreicht.

Eine weitere Annahme der sozial-kognitiven Theorie über die Merkmale selbstregulierten Lernens bezieht sich auf die Möglichkeit von Personen, als proaktiv Lernende den Prozess des Erwerbs von Fähigkeiten und Fertigkeiten aktiv zu beeinflussen und zu gestalten (Bandura, 1986). Diese Auffassung bedeutet für die Praxis, dass Schüler(innen) zunächst einmal das Bewusstsein erlangen müssen, dass sie Verantwortung für das eigene Lernen übernehmen können und Handlungsspielraum besitzen (z.B. bei der Wahl adäquater Lernstrategien oder der Art und Weise, wie sie ihre Hausaufgaben erledigen). Ein solches Verständnis kann nicht als gegeben vorausgesetzt werden, sondern erfordert eine entsprechende Vermittlung. Ansätze zur Förderung selbstregulierten Lernens stellen günstige Gelegenheiten dar, ein Bewusstsein dafür zu schaffen, dass Wissenserwerb kein passiver Prozess ist und dass die eigene Leistungsentwicklung zu einem entscheidenden Anteil in der Verantwortung jeder und jedes Lernenden liegt. Die Vermittlung sollte dabei stets kontextspezifisch, d.h. bezogen auf entsprechende fachbezogene Inhalte, geschehen. Sie sollte zudem Informationen darüber enthalten, wie diese Verantwortungsübernahme für und Kontrolle über den eigenen Lernprozess konkret und auch unter weitgehend strukturierten Bedingungen (regulärer Unterricht und Erledigung von Hausaufgaben) aussehen kann. Die Mitgestaltung des Wissenserwerbs setzt auf Seiten der Lernenden darüber hinaus eine Sensibilität für persönliche Stärken und Schwächen, also Kenntnisse der eigenen Fähigkeiten (d.h. metakognitives Wissen) voraus. Für die Förderung von Selbstregulation bedeutet dies, Schüler(innen) darin zu unterstützen ihre metakognitiven Kompetenzen ${ }^{3}$ und die Überwachung eigenen Lernens und Arbeitens (self-monitoring) auf- und auszubauen. Ein solches Bewusstsein gibt ihnen die Möglichkeit entsprechend der sozial-kognitiven Vorstellung proaktiv und im Hinblick auf das Erreichen persönlicher Ziele (Zimmerman, 2000) zu lernen.

Für die Entwicklung von Interventionsansätzen spielt die Annahme, dass es sich bei selbstreguliertem Lernen nicht um eine statische mentale Fähigkeit oder akademische Leistungskomponente, sondern um einen zyklischen Prozess aus drei Phasen (Vorbereitungsphase, Handlungsphase und Selbstreflexionsphase; Zimmerman, 2000) handelt, eine zentrale Rolle:

\footnotetext{
3 Die Verwendung des Kompetenzbegriffes erfolgt in Anlehnung an Klieme et al. (2003) bzw. Weinert (2001, S. 27f.): Kompetenzen werden verstanden als die bei Individuen verfügbaren oder von ihnen erlernbaren kognitiven Fähigkeiten und Fertigkeiten, bestimmte Probleme zu lösen, sowie die damit verbundenen motivationalen, volitionalen und sozialen Bereitschaften und Fähigkeiten, die Problemlösungen in variablen Situationen erfolgreich und verantwortungsvoll nutzen zu können.
} 
Erstens impliziert diese Auffassung die grundsätzliche Veränderbarkeit der Selbstregulationsfähigkeit und damit die Möglichkeit, durch Interventionen fördernd Einfluss zu nehmen. Zweitens bietet diese Konzeption an verschiedenen Stellen des Prozesses Ansatzpunkte für die Förderung, so dass etwa Subprozesse (z.B. Zielsetzung und Planung, Self-monitoring, Selbstreflexion) fokussiert werden können. Zimmerman (1990) weist jedoch darauf hin, dass Interventionen, die nicht das Zusammenspiel metakognitiver, motivationaler und behavioraler Faktoren im Selbstregulationsprozess berücksichtigen, wenig Aussicht auf langfristigen Erfolg haben (z.B. ist ein isoliertes Training von Lernstrategien wenig effektiv, wenn Lernende nicht zugleich motiviert sind, diese tatsächlich anzuwenden). Bei Überlegungen zur Förderung sollte daher - unabhängig von der Bandbreite des Inhalts - stets die zyklische Natur der Selbstregulation berücksichtigt werden. Eine Auswahl von trainierten Selbstregulationskomponenten muss dabei keinen Widerspruch zur Betrachtung des gesamten Zyklus darstellen.

Zusammenfassend lassen sich aus den Grundprinzipien und Annahmen der sozialkognitiven Theorie folgende Implikationen für die Förderung selbstregulierten Lernens ableiten (vgl. Tab. 3.1): Das Konzept des reziproken Determinismus betont die wechselseitige Beziehung von Person, Verhalten und (Lern-) Umwelt und hebt hervor, dass Veränderungen innerhalb jedes einzelnen Faktors Auswirkungen auf die anderen haben können. Förderung findet also nie isoliert, sondern stets innerhalb eines komplexen Bedingungsgefüges statt, in dem soziale Einflüsse einen entscheidenden Einfluss auf den Lernprozess haben. Die Formulierung von Entwicklungsniveaus der Selbstregulationsfähigkeit unterstreicht die Relevanz von Verhaltensmodellen sowie der externen Anleitung und Unterstützung zu Beginn des Fähigkeitserwerbs und der zunehmenden Autonomie der lernenden Person bei steigender Elaboriertheit. Aus der Annahme proaktiv Lernender geht hervor, dass Schüler(inne)n sowohl ihre Verantwortung für den eigenen Lernprozess und in direkter Verbindung damit auch die konkreten Möglichkeiten der Umsetzung aufgezeigt werden sollten. Die Vermittlung metakognitiver Fähigkeiten wie self-monitoring spielt eine wichtige Rolle für den Aufbau von Selbstkontrolle des Fähigkeits- und Fertigkeitserwerbs. Die Betrachtung selbstregulierten Lernens als einen zyklischen Prozess in Abgrenzung zu einer stabilen Persönlichkeitseigenschaft bedeutet für die Förderung, dass Interventionen an verschiedenen Punkten des Zyklus ansetzen können, gleichzeitig jedoch die Interaktion der Subprozesse zu berücksichtigen ist. Die Prozessperspektive sollte grundsätzlich die Basis sozial-kognitiv orientierter Interventionsansätzen darstellen, unabhängig davon, welche und wieviele Selbstregulationskomponenten gefördert werden. 
Tabelle 3.1. Elemente der sozial-kognitiven Theorie, Implikationen und praktische Beispiele

\begin{tabular}{|c|c|c|}
\hline Element der Theorie & Implikation & praktisches Beispiel \\
\hline $\begin{array}{l}\text { Reziproker } \\
\text { Determinismus }\end{array}$ & $\begin{array}{l}\text { Selbstreguliertes Lernen ist kein im } \\
\text { Individuum isoliert stattfindender } \\
\text { Prozess } \\
\text { soziale Einflüsse haben entscheiden- } \\
\text { den Einfluss auf den Lernprozess } \\
\end{array}$ & $\begin{array}{l}\text { Lernende dienen sich ge- } \\
\text { genseitig als Verhaltensmo- } \\
\text { delle } \\
\text { Einflüsse sozialen Ver- } \\
\text { gleichs im Klassenraum }\end{array}$ \\
\hline $\begin{array}{l}\text { Entwicklungsniveaus } \\
\text { der Selbstregulation }\end{array}$ & $\begin{array}{l}\text { Relevanz von Verhaltensmodellen, } \\
\text { genauer Anleitung und Feedback zu } \\
\text { Beginn des Lernprozesses } \\
\text { Übungsmöglichkeiten und Autono- } \\
\text { mie im Laufe der Fähigkeitsentwick- } \\
\text { lung }\end{array}$ & $\begin{array}{l}\text { Lehrkraft dient als Verhal- } \\
\text { tensmodell, z.B. bei der } \\
\text { schrittweisen Anwendung } \\
\text { einer Bearbeitungsstrategie } \\
\text { Übungs- und Anwendungs- } \\
\text { phasen einplanen }\end{array}$ \\
\hline $\begin{array}{l}\text { Annahme proaktiv } \\
\text { Lernender }\end{array}$ & $\begin{array}{l}\text { Bedeutung der Verantwortungsüber- } \\
\text { nahme seitens der Lernenden bei } \\
\text { gleichzeitiger Vermittlung und Be- } \\
\text { reitstellung von Möglichkeiten der } \\
\text { konkreten Umsetzung } \\
\text { Förderung von metakognitiven Fä- } \\
\text { higkeiten wie self-monitoring }\end{array}$ & $\begin{array}{l}\text { Bedeutung und Nutzen ver- } \\
\text { schiedener Strategien expli- } \\
\text { zit thematisieren } \\
\text { - Mit Lernenden über adäqua- } \\
\text { te Strategien diskutieren } \\
\text { Selbstinstruktionen zur } \\
\text { Förderung metakognitiver } \\
\text { Kontrolle }\end{array}$ \\
\hline $\begin{array}{l}\text { SRL als zyklischer } \\
\text { Prozess }\end{array}$ & $\begin{array}{l}\text { Förderung kann an verschiedenen } \\
\text { Stellen des Selbstregulationsprozes- } \\
\text { ses ansetzen } \\
\text { Trainings sollten metakognitive, mo- } \\
\text { tivationale und behaviorale Kompo- } \\
\text { nenten fördern }\end{array}$ & $\begin{array}{l}\text { z.B. Förderung von Zielset- } \\
\text { zung, diese verbinden mit } \\
\text { einer anschließenden Refle- } \\
\text { xion über Resultate und } \\
\text { Rückbezug auf Ziele }\end{array}$ \\
\hline
\end{tabular}

\subsection{Ansätze zur Förderung selbstregulierten Lernens}

Die zahlreichen Belege für die Bedeutung selbstregulierten Lernens im Hinblick auf Schulleistung und den Erwerb akademisch relevanter Fähigkeiten und Fertigkeiten (z.B. Schunk, 1994; Zimmerman \& Bandura, 1994; Zimmerman \& Martinez Pons, 1986, 1988, 1990) führten in den vergangenen Jahren verstärkt zur Entwicklung von Trainingsprogrammen zur Förderung der Selbstregulation von Schüler(inne)n (Schunk \& Ertmer, 2000; Schunk \& Zimmerman, 1998). Forschungsprojekte solcher Art verfolgen zumeist zwei zentrale Ziele: Zum einen ermöglichen (quasi-) experimentelle Interventionsstudien eine kritische statistische Überprüfung des Zusammenhangs zwischen Selbstregulationsfähigkeiten und fachbezogenen Schulleistungen, deren Aussagekraft über korrelative Studien (z.B. Zimmerman \& MartinezPons, 1986) hinausgeht. Theoretische Annahmen (z.B. basierend auf der Theorie nach Zimmerman, 2000) werden auf diese Weise empirisch prüfbar. Das zweite Ziel von Programm- 
evaluationen ist primär praktischer Natur: Aufgrund des angenommenen engen Zusammenhangs zwischen Selbstregulation und Leistung sollte der Aufbau von Selbstregulationsfähigkeiten das Lernvermögen und damit die schulischen Erfolge verbessern. Selbstregulationsbezogene Interventionsforschung verfolgt daher auch das Ziel, konkrete Umsetzungsmöglichkeiten für die Schulpraxis zu konzipieren und zu evaluieren. Die Entwicklung der Programme erfolgt häufig basierend auf der sozial-kognitiven Theorie (Bandura, 1986; Zimmerman, 2000), die einen geeigneten Rahmen für Ansätze zur Implementation von Förderung in den Schulkontext darstellt. Entsprechend ist eine Leitlinie der meisten in den letzten Jahren erfolgreich evaluierten Trainingsprogramme die kontextspezifische Förderung, umgesetzt durch die Verknüpfung der Selbstregulationsinstruktion mit curricular validen Inhalten (vgl. Hasselhorn \& Labuhn, 2008). Außerdem basieren die Interventionen in der Regel auf einem zyklischen Selbstregulationsmodell (Zimmerman, 2000 oder Pintrich, 2000; Schmitz, 2001; Schmitz \& Wiese, 2006), und selbst bei der fokussierten Förderung einzelner Komponenten wird bei der Konzeption der gesamte Selbstregulationsprozess berücksichtigt.

Ein Beispiel für einen prozessorientierten, kontextspezifischen Ansatz stellt das von Perels, Otto, Landmann, Hertel und Schmitz (2007) sowie Perels, Gürtler und Schmitz (2005) präsentierte Training dar, welches erfolgreich den gesamten Zyklus der Selbstregulation mit mathematischem Problemlösen kombiniert. Auch den Elzen-Rump und Leutner (2007) sowie Leopold, den Elzen-Rump und Leutner (2006) berichten über die erfolgreiche Integration der Förderung fach- und selbstregulationsbezogener Inhalte. In der Untersuchung im Rahmen des naturwissenschaftlichen Unterrichts wurde zunächst der selbstregulierte Einsatz von Lern- und Textbearbeitungsstrategien durch ein computerbasiertes Training eingeführt und die Schüler(innen) anschließend im Unterricht zum Gebrauch der Strategien aufgefordert. Ein weiteres Beispiel für prozessorientierte, kombinierte fach- und selbstregulationsbezogene Förderung stellt das „Self-Regulation Empowerment Program“ (Cleary \& Zimmerman, 2004) dar. In individueller Arbeit mit einzelnen Schüler(inne)n wird das Ziel verfolgt, Lernende an die Möglichkeiten der Selbstregulation heranzuführen, d.h. sie bei Zielsetzung, Selbstbeobachtung und Selbstbewertung, der Auswahl und Anpassung von Lernstrategien sowie der Verbesserung der Lernleistung begleitend zu unterstützen. Dieses Programm, sowie die Trainings von Perels et al. (2005, 2007), den Elzen-Rump und Leutner (2007) und Leopold, den ElzenRump und Leutner (2006) fanden zwar im Schulkontext, jedoch nicht im regulären Unterricht statt und ließen die Frage nach der Wirksamkeit der Integration von Förderung in den Fachunterricht zunächst offen. Die zahlreichen Hinweise auf die Effektivität der Verknüpfung selbstregulierten und fachlichen Lernens führten jüngst zu wachsendem Forschungsinteresse an der 
systematischen Einbindung der Selbstregulationsförderung in den Fachunterricht. Für das Fach Mathematik zeigen Fuchs et al. (2003), dass sich die Förderung selbstregulierten Lernens mit der Vermittlung mathematischer Strategien im regulären Unterricht verbinden lässt und die Lernleistung positiv beeinflusst. Ein weiteres Beispiel für die Effektivität des unterrichtsintegrierten Ansatzes geben Perels, Dignath und Schmitz (in Druck) mit ihrer prozessorientierten Interventionsstudie, ebenfalls innerhalb des Mathematikunterrichts. Souvignier und Mokhlesgerami (2006) berichten über die Wirksamkeit des in den regulären Unterricht integrierten, selbstregulationsorientierten Trainingsansatzes von Schreblowski und Hasselhorn (2001) zur Förderung des Leseverständnisses. Glaser und Brunstein (2007a, 2007b) belegen die Effektivität eines Schreibtrainings, bei dem im Rahmen des regulären Deutschunterrichts die Vermittlung von Strategien zur Textrevision mit dem Training von Selbstregulationsstrategien kombiniert wurde.

Die Ergebnisse dieser üblicherweise quasi-experimentell angelegten Studien belegen übereinstimmend, dass selbstreguliertes Lernen durch eine Intervention gefördert (vgl. auch Schunk \& Ertmer, 2000; Schunk \& Zimmerman, 1998) und dass die Förderung kontextspezifisch im regulären Unterricht realisiert werden kann. Die evaluierten unterrichtsintegrierten Programme fanden bislang in erster Linie im Rahmen des Mathematik- oder Deutschunterrichts statt und bezogen sich auf stark strukturierte, homogene fachbezogene Inhalte wie mathematische Problemlösestrategien (Fuchs et al., 2003; Perels, Dignath \& Schmitz, in Druck), Textrevisionsstrategien (Glaser \& Brunstein, 2007a, 2007b) oder Strategien zur Förderung des Leseverständnis (Souvignier \& Mokhlesgerami, 2006; nach Schreblowski \& Hasselhorn, 2001). Die Inhalte des auf den Bereich der Naturwissenschaften bezogenen Selbstregulationstrainings (den Elzen-Rump \& Leutner, 2007; Leopold, den Elzen-Rump \& Leutner, 2006) sollten zwar von den Schüler(inne)n im regulären Unterricht angewandt werden, die Umsetzung fand jedoch außerhalb dessen in einem computerbasierten Setting statt (s.o.). Damit bleibt die Frage nach der kurz- und langfristigen Wirksamkeit der Selbstregulationsförderung, die a) innerhalb des regulären naturwissenschaftlichen Unterrichts stattfindet, b) sich auf eine curricular valide Unterrichtseinheit mit heterogenen Inhaltskomponenten bezieht und c) in enger Kooperation mit Fachlehrkräften entwickelt und von diesen durchgeführt wird, zunächst offen. Meines Wissens liegen darüber hinaus bislang keine Befunde zur differentiellen Wirkung des unterrichtsintegrierten Ansatzes, z.B. für Subgruppen mit unterschiedlichen Lernvoraussetzungen, vor.

Im Hinblick auf die Implikationen der sozial-kognitiven Theorie stellt sich weiterhin die Frage, welche Möglichkeiten der unterrichtsintegrierten Selbstregulationsförderung über 
die Umsetzung sorgfältig geplanter Unterrichtsprogramme hinaus bestehen. Es wäre wünschenswert, Merkmale von Instruktion und der Lernumwelt, welche den Aufbau von Selbstregulationsfähigkeiten in Schüler(inne)n unterstützen, zu benennen und empirisch zu überprüfen. Da dem gezielten Training erfolgreichen Lernverhaltens vielerorts ein Mangel an Zeit und finanziellen Ressourcen entgegensteht (vgl. Schunk \& Zimmerman, 1998), könnte die Identifikation solcher instruktions- oder kontextbezogener Variablen dazu beitragen, mehr Lehrkräfte zu motivieren geringfügige, aber effektive (und theoriegeleitete) Modifikationen des Unterrichtsgeschehens vorzunehmen (im Gegensatz zur Implementation komplexer Trainings). Auch auf diese Weise könnten Schüler(innen) in ihrer Entwicklung zu proaktiv und selbstreguliert Lernenden unterstützt werden.

\subsection{Forschungsfragen}

Basierend auf den Implikationen der sozial-kognitiven Theorie für die Förderung selbstregulierten Lernens und den bislang ungeklärten Aspekten im Zusammenhang mit Selbstregulationsförderung im Schulkontext wurden für die vorliegende Arbeit folgende Forschungsfragen formuliert:

1. Lässt sich selbstreguliertes Lernen unterrichtsintegriert ohne negative Auswirkungen auf den kurzfristigen fachlichen Wissenserwerb wirksam fördern?

2a. Führt eine zeitlich begrenzte unterrichtsintegrierte Selbstregulationsförderung längerfristig zu einem Aufbau der Selbstregulationsfähigkeit?

2b. Führt eine zeitlich begrenzte unterrichtsintegrierte Selbstregulationsförderung zu längerfristigen positiven Effekten auf den Lernerfolg?

3. Ist die unterrichtsintegrierte Selbstregulationsförderung für Schüler(innen) mit unterschiedlichen Lernvoraussetzungen gleich wirksam?

4. Welche Merkmale von Instruktion und Einflüssen des sozialen Kontextes im Klassenraum unterstützen den Aufbau von Selbstregulation?

In den empirischen Beiträgen, präsentiert in den Kapiteln 4.1, 4.3 und 6, werden die Forschungsfragen weiter ausdifferenziert. Im abschließenden Kapitel 7 erfolgt eine Integration und Diskussion der empirischen Befunde zu den aufgeworfenen Fragen. 


\section{Integration der Selbstregulationsförderung in den naturwissenschaftli- chen Fachunterricht (Studie 1)}

\subsection{Lernförderung durch Anregung der Selbstregulation im naturwissenschaftlichen Unterricht $^{4}$}

\subsubsection{Zusammenfassung und Abstract}

In einer quasi-experimentellen Interventionsstudie wurde die Wirksamkeit einer Verzahnung von selbstreguliertem Lernen mit naturwissenschaftlichem Fachunterricht untersucht. In enger Kooperation mit Lehrkräften wurde eine Unterrichtseinheit zum Thema Ernährung konzipiert und entweder mit oder ohne zusätzliche Anregungen zum selbstregulierten Lernen mit 199 Schüler(inne)n der siebten Jahrgangsstufe einer Gesamtschule durchgeführt. Die Resultate des Prätest-Posttest-Vergleichs zeigen, dass die zusätzlichen Selbstregulationsanregungen zu einem Anstieg selbstregulierten Lernens führen. Trotz reduzierter Unterrichtszeit für fachbezogene Inhalte wurde unter der Bedingung mit Selbstregulationsanregungen fachlich ebenso viel gelernt wie in der Vergleichsgruppe. In einer anschließenden neuen und für beide Gruppen identischen Unterrichtseinheit konnte darüber hinaus ein Vorteil der Trainingsgruppe bezüglich des Lernerfolgs nachgewiesen werden.

\section{Abstract}

This article addresses the connection of self-regulation and the acquisition of content knowledge in science education. A quasi-experimental design was used to investigate the connection between self-regulation and the acquisition of content knowledge in science education of 199 seventh graders in a German comprehensive school. The core of the study built a unit on the topic of nutrition that was designed in conjunction with the local teachers. Whereas students in the control group received regular instruction on the subject matter, teachers in the experimental group infused central processes of self-regulation in their classroom instruction. To assess transfer of self-regulatory processes, a subsequent unit was conducted to both groups simultaneously. The results confirm that self-regulated learning can be enhanced in classrooms through this brief intervention. Although it seemed that the teaching of self-regulatory processes during the learning unit took away time from the actual presenta-

\footnotetext{
${ }^{4}$ Dieses Teilkapitel ist bereits erschienen: Labuhn, A. S., Bögeholz, S. \& Hasselhorn, M. (2008). Lernförderung durch Anregung der Selbstregulation im naturwissenschaftlichen Unterricht, Zeitschrift für Pädagogische Psychologie, 22, 13-24.
} 
tion of content knowledge, trained students scored as high as students from the control group. In addition, subsequent learning in the second teaching unit was improved by the preceding intervention.

\subsubsection{Einleitung}

Nicht nur für schulisches Lernen ist ein hohes Maß an Flexibilität bei der Wissensaneignung und der Einstellung auf neue, komplexe Themengebiete erforderlich. Das Vermögen, Lernprozesse selbst zu initiieren und zu regulieren spielt dabei eine wichtige Rolle. Zimmerman (2002) sieht im Aufbau individueller Voraussetzungen für lebenslanges Lernen eine zentrale Funktion von Bildung. Lehrer(innen) sollten nicht nur über die individuellen Stärken und Schwächen der einzelnen Schüler(innen) Bescheid wissen, sondern sie darüber hinaus in die Lage versetzen, ein Bewusstsein für die eigenen Fähigkeiten und deren Weiterentwicklung aufzubauen (Zimmerman, 2002). Im Rahmen der PISA-Studie (Artelt, Baumert \& Julius-McElvany, 2003) durchgeführte Lehrplananalysen zeigen, dass der Aufbau der Fähigkeit zum selbstregulierten Lernen (SRL) in der Bundesrepublik Deutschland zu den expliziten Zielen gehört. Klieme und Stanat (2002) stellen jedoch fest, dass problemorientiertes, verständnisintensives Lernen zugunsten einer Orientierung an der Reproduktion von Faktenwissen in der Schulpraxis vernachlässigt wird. Innovationen werden unter anderem deshalb abgelehnt, weil Lehrer(innen) sie als wenig relevant oder nützlich beurteilen (Blumenfeld, Fishman, Krajcik \& Marx, 2000). Dem gezielten Training erfolgreichen Lernverhaltens steht auBerdem nicht selten ein Mangel an Zeit und finanziellen Ressourcen entgegen (Schunk \& Zimmerman, 1998). Souvignier und Mokhlesgerami (2006) kommen zu der Einschätzung, dass die Unterrichtsqualität unter anderem dadurch verbessert werden könnte, dass Lehrer(inne)n entsprechend ausgearbeitete Materialien zur Verfügung gestellt würden, die zentrale Komponenten der Selbstregulation berücksichtigen. Eine Metaanalyse brachte zudem das Ergebnis hervor, dass die Wirksamkeit von innovativen Unterrichtsansätzen im Schulalltag besonders gut ausfällt, wenn die Implementation in enger Kooperation mit Forscherteams vorgenommen wird (Souvignier \& Antoniou, 2007). Im Rahmen der vorliegenden Studie erstellten daher Lehrer(innen) und Wissenschaftler(innen) gemeinsam eine Unterrichtseinheit mit gleichzeitiger Sachwissens- und Selbstregulationsvermittlung. 


\section{Selbstregulation als zyklischer Prozess}

In der einschlägigen Literatur wird selbstreguliertes Lernen weitgehend übereinstimmend als ein dynamisches Zusammenwirken von kognitiven, metakognitiven und motivationalen Aspekten des Lernens verstanden (z.B. Boekaerts, 1999; Schmitz, 2001; Zimmerman, 2000, 2002). Die in diesem Artikel vorgestellte Unterrichtsintervention baut auf der Modellvorstellung Zimmermans (2000, 2002) auf. Danach wird Selbstregulation aus einer sozialkognitiven Perspektive als ein individueller Prozess betrachtet, was im Gegensatz zur Annahme einer statischen mentalen Fähigkeit oder akademischen Leistungskomponente Ansatzpunkte für Förderung und Intervention an verschiedenen Punkten des Prozesses bietet. Zimmerman beschreibt selbstreguliertes Lernen im Rahmen dreier zyklischer Phasen: Vorbereitungsphase, Handlungsphase und Selbstreflexionsphase. In der Vorbereitungsphase laufen Prozesse ab, die der Aufgabenbearbeitung vorangehen. Während dieser Phase sind Planung und Zielsetzung sowie verschiedene Quellen von Motivation, z.B. Selbstwirksamkeitsüberzeugungen, von Bedeutung. Die zentralen Prozesse der Handlungsphase beziehen sich direkt auf die Bearbeitung der Aufgabe. Hier sind der Einsatz von Lernstrategien sowie die Fähigkeit, den eigenen Arbeitsprozess zu beobachten (self-monitoring), entscheidende Voraussetzungen für den Lernerfolg. Darüber hinaus muss die Aufmerksamkeit nicht nur auf die Bearbeitung der Aufgabe fokussiert, sondern auch über einen längeren Zeitraum aufrechterhalten und gegen Ablenkung abgeschirmt werden. Hier spielen u.a. volitionale Strategien eine wichtige Rolle. Die Selbstreflexionsphase schließt sich der Handlung an und bezieht sich inhaltlich direkt auf das Resultat der Aufgabenausführung. Die Reflektion über das erzielte Ergebnis umfasst einen Abgleich mit der persönlichen Zielsetzung sowie die Bewertung der eigenen Leistung und nimmt Einfluss auf den folgenden Selbstregulationszyklus.

\section{Selbstreguliertes Lernen und Schulleistungen}

Im Rahmen der Planung des eigenen Lernens spielt die persönliche Zielsetzung eine entscheidende Rolle. Wie Locke und Latham (1990) zeigen konnten, wirken konkrete, spezifische, zeitnahe sowie anspruchsvolle und zugleich erreichbare Ziele positiv auf das Erreichen hoher Leistungen. Neben der Zielsetzung kommt in der Vorbereitungsphase auch motivationalen Variablen Bedeutung zu. Zimmerman und Bandura (1994) fanden positive Zusammenhänge zwischen der Selbstwirksamkeitserwartung einerseits und dem Setzen von Zielen, der Selbstbewertung und der tatsächlich erbrachten Leistung andererseits. Pintrich und DeGroot (1990) untersuchten die Beziehungen zwischen Selbstregulation, Selbstwirksamkeitserwar- 
tung und Schulleistung bei Siebtklässler(inne)n in den Fächern Naturwissenschaften und Englisch. Die Selbstwirksamkeitserwartung, der Einsatz kognitiver Strategien und die Selbstregulation korrelierten positiv untereinander und sagten Schulleistung vorher. Das Self-monitoring des Strategieeinsatzes in der Handlungsphase bewirkte in einer Studie von Zimmerman und Kitsantas (1999) eine Steigerung der Selbstwirksamkeit sowie des untersuchten schriftlichen Ausdrucksvermögens. Im Hinblick auf die strategischen Prozesse der Handlungsphase soll an dieser Stelle auch auf die eher widersprüchliche Befundlage zur Beziehung von Lernstrategien und Schulleistung hingewiesen werden. Häufig wurden lediglich schwache Zusammenhänge gefunden (z.B. Baumert, 1993). Gründe für solche Resultate werden in der kontextunabhängigen Formulierung von Items in Lernstrategiefragebögen und der Ungenauigkeit retrospektiver Selbstberichte gesehen. Handlungsnah angelegte empirische Studien (vgl. Artelt, 1999; Cleary \& Zimmerman, 2004) weisen dagegen darauf hin, dass grundsätzlich ein substanzieller Zusammenhang zwischen dem Einsatz adäquater Lernstrategien und Schulleistung angenommen werden kann.

\section{Förderung selbstregulierten Lernens}

Durch experimentell angelegte Studien konnte bereits mehrfach gezeigt werden, dass selbstreguliertes Lernen durch eine Intervention gefördert werden kann (vgl. Schunk \& Ertmer, 2000; Schunk \& Zimmerman, 1998). Trainingsmaßnahmen, die sich auf schulisches Lernen beziehen, haben sich grundsätzlich als effektiv erwiesen, fanden aber zunächst häufig außerhalb des regulären Unterrichts statt (z.B. Perels, Gürtler \& Schmitz, 2005) oder wurden in individueller Arbeit mit einzelnen Lernenden realisiert (z.B. Cleary \& Zimmerman, 2004). Perels, Gürtler und Schmitz (2005) berichten über ein prozessorientiertes Training, welches erfolgreich Selbstregulation mit mathematischem Problemlösen kombiniert. Die Tatsache, dass zentrale Aspekte der Selbstregulation mit Lernerfolg kovariieren, wirft die Frage auf, inwiefern Unterricht, der selbstreguliertes Lernen systematisch fördert, auch eine Verbesserung der fachbezogenen Leistung bewirken kann. Souvignier und Mokhlesgerami (2006) fanden in einer schulischen Umsetzung des Trainingsansatzes von Schreblowski und Hasselhorn (2001) zur Förderung des Leseverständnisses, dass Unterrichtsvarianten, die sowohl kognitive als auch motivationale Komponenten der Selbstregulation integrierten, langfristig zu besserem Lernerfolg führten als Varianten, die lediglich einzelne (z.B. nur kognitive) Aspekte berücksichtigten. Auch den Elzen-Rump und Leutner (2007) sowie Leopold, den Elzen-Rump und Leutner (2006) befassten sich mit der Integration eines Förderprogramms in den Schulalltag. In ihrer Untersuchung im Rahmen des naturwissenschaftlichen Unterrichts der zehnten 
Jahrgangsstufe wurden zunächst der Einsatz von Lernstrategien und deren selbstregulierte Anwendung durch ein computerbasiertes Training eingeführt. Die Aufgabe der teilnehmenden Lehrer(innen) bestand anschließend darin, die Schüler(innen) zum Gebrauch der Lernstrategien im Unterricht anzuregen. Auf diese Weise trainierte Schüler(innen) zeigten im Vergleich zu einer Kontrollgruppe anschließend bessere Leistungen beim Textverstehen. In einer Studie von Fuchs et al. (2003) wird der mögliche Interventionserfolg besonders deutlich. Die Autor(innen) untersuchten die Wirksamkeit von Unterrichtsansätzen zur Vermittlung von mathematischem Problemlösen und von Selbstregulation im Schulalltag. Eine Kombination aus Problemlöseund Selbstregulationsförderung zeigte sich den anderen Varianten hinsichtlich der Selbstregulation der Schüler(innen) sowie der fachbezogenen Leistung überlegen. Es konnte darüber hinaus ein Transfereffekt des selbstregulierten Lernens auf die Leistungen in einem Mathematiktest nachgewiesen werden, der sich in Form und Inhalt von den während der Intervention eingesetzten Materialien unterschied. Für den Schulkontext erscheint der Transfer lernförderlicher Fähigkeiten besonders wichtig zu sein. Deren Vermittlung wäre von besonderem Wert, wenn sie auch das Lernen in anderen Themengebieten begünstigen würde. Pressley et al. (1990) gehen davon aus, dass der Transfer selbstregulierten Lernens auf andere Kontexte, Inhalte und Aufgaben von mindestens drei Faktoren abhängt: a) von dem Wissen über Selbstregulation, b) von der Überzeugung, dass Selbstregulation nützlich ist und c) von der Fähigkeit, den Selbstregulationsprozess der Situation entsprechend anzupassen. Wenn es gelingt, diese Bereiche durch entsprechende Instruktion gezielt zu fördern und Transfereffekte zu erzielen, würde der Nutzen der unterrichtsintegrierten Förderung zusätzlich gesteigert.

\subsubsection{Fragestellung}

Vor dem Hintergrund dieser Befunde war das Ziel der vorliegenden Studie, die systematische Anregung verschiedener Elemente der Selbstregulation in den naturwissenschaftlichen Unterricht zu integrieren und zu prüfen, ob sich dadurch die Selbstregulation steigern lässt und wie sich dieses Vorgehen auf das fachliche Lernen auswirkt. Zunächst ging es um die Frage, ob die Intervention im engeren Sinne wirksam ist und zu einer Verbesserung der Selbstregulationsfähigkeit führt. Außerdem sollte geklärt werden, wie sich die Intervention auf die fachbezogene Lernleistung auswirkt. Da Selbstregulationsanregungen Unterrichtszeit beanspruchen, die dann nicht mehr für die unmittelbare Vermittlung von Fachinhalten zur Verfügung steht, wären durchaus auch negative Effekte auf die fachbezogene Lernleistung denkbar. Darüber hinaus gibt es empirische Belege, die darauf hindeuten, dass Lernende nach 
der Einführung neuer Strategien kurzfristig in ein „Motivationstal“ geraten und die Leistung zunächst absinkt (Hasselhorn \& Gold, 2006, S. 97ff.). Die dritte Frage bezog sich schließlich darauf, ob durch unterrichtsintegrierte Selbstregulationsanregung ein Lernvorteil in späteren Unterrichtseinheiten erzielt werden kann.

\subsubsection{Methode}

Design

Um die aufgeworfenen Fragen für eine konzipierte unterrichtsintegrierte Selbstregulationsanregung empirisch klären zu können, wurde eine abgewandelte Variante des PrätestPosttest-Kontrollgruppendesigns verwendet. Die an der Studie teilnehmenden Klassen wurden auf zwei experimentelle Bedingungen aufgeteilt: Trainingsgruppe (TG; Unterricht mit Anregung der Selbstregulation) und Kontrollgruppe (KG; Unterricht ohne Selbstregulationsanregung). Die Klassen, deren Lehrer(innen) sich bereit erklärten, die Selbstregulationsförderung in den Unterricht zu integrieren, bildeten die Trainingsgruppe. In Anlehnung an einen Versuchsplan von Klauer (1997) ist die Untersuchung durch zwei Phasen gekennzeichnet. Lediglich in der Phase I wurde die unabhängige Variable „Selbstregulationsanregung“ variiert. In der Trainingsgruppe fand eine achtstündige Unterrichtseinheit im Fach Naturwissenschaften statt, in die Anregungen der Selbstregulation integriert wurden. Die Kontrollgruppe wurde zu dem gleichen Thema ohne explizite Anregung selbstregulierten Lernens ebenfalls acht Stunden lang unterrichtet. In der Phase II folgte eine in allen Klassen identisch durchgeführte fünfstündige Unterrichtseinheit ohne eine explizite Einbindung von Selbstregulationselementen. Die Phase II wurde realisiert, um die Auswirkungen der Selbstregulationsanregung auf eine neue Unterrichtseinheit zu explorieren.

\section{Stichprobe}

Die Studie wurde in einer integrierten Gesamtschule in Nordrhein-Westfalen durchgeführt. Es nahmen alle sieben Klassen des siebten Jahrgangs teil. Die Trainingsgruppe bestand aus vier, die Kontrollgruppe aus drei Klassen. Zum ersten Messzeitpunkt umfasste die Stichprobe $\mathrm{N}=199$ Schüler(innen) (TG: 115; KG: 84), davon 94 Mädchen und 105 Jungen. Für den zweiten Messzeitpunkt liegen 185 (TG: 107; KG: 78) und für den dritten Messzeitpunkt 172 (TG: 100; KG: 72) komplette Datensätze vor. Das Alter der Schüler(innen) betrug zum ersten Messzeitpunkt im Durchschnitt 13;2 Jahre ( $\mathrm{SD}=0.6$ ); 39.2 Prozent von ihnen stammen aus Familien mit Migrationshintergrund. Trainings- und Kontrollgruppe unterschieden sich 
nicht hinsichtlich Alter (TG: 13.25; KG: 13.24), Geschlecht (TG: 46\% Mädchen; KG: 48\% Mädchen), Anzahl der Schüler(innen) mit Migrationshintergrund (TG: 39\%; KG: 40\%) und sprachlichen Fähigkeiten, gemessen durch die Verbalskala V1 des KFT (Heller \& Perleth, 2000) (TG: 11.94; KG: 11.98).

\section{Konzeption und Umsetzung der Unterrichtsvarianten}

Die Untersuchung wurde im Fach Naturwissenschaften durchgeführt. Bei der Unterrichtsentwicklung orientierten wir uns am symbiotischen Implementationsansatz (Gräsel \& Parchmann, 2004) und arbeiteten folglich eng mit den Lehrkräften zusammen. Erstellt wurde eine lehrplanmäßig vorgesehene Unterrichtseinheit zum Thema „Ernährung“. Die Inhalte dieser achtstündigen Einheit wurden von den Lehrer(inne)n aller teilnehmenden Klassen gemeinsam mit der Erstautorin ausgearbeitet. Die Planung wurde in Form von Stundenverlaufsplänen festgehalten, um eine Grundlage zur Kommunikation über die Konzeption der Stunden sowie deren Durchführung zu schaffen. Ergebnis der gemeinsamen Unterrichtsentwicklung war ein so genannter Basis-Unterricht, der in der Kontrollgruppe zum Einsatz kam. Für die Trainingsgruppe erstellte die Erstautorin eine mit Elementen der Selbstregulationsförderung angereicherte Variante dieses Basis-Unterrichts (vgl. Tabelle 4.1). Die Auswahl der Trainingselemente in der vorliegenden Studie erfolgte unter Berücksichtigung aller drei Phasen des Selbstregulationsmodells. In der Intervention werden besonders Zielsetzung und Motivation (Vorbereitungsphase), Self-monitoring, volitionale Strategien und Lernstrategien (Handlungsphase) sowie die Einschätzung des eigenen Lernergebnisses (Selbstreflexionsphase) fokussiert. Bezüglich der Inhalte der Selbstregulationsförderung wurde außerdem auf bereits bestehende und evaluierte Trainingskonzepte zurückgegriffen (vgl. Perels, Gürtler \& Schmitz, 2005; Perels, Schmitz \& Bruder, 2003).

Um Selbstregulation in den Unterricht zu integrieren, wurden die Stundeninhalte des Basis-Unterrichts auf ca. 30 min verdichtet. Damit wurden für jede Unterrichtsstunde 15 min für das Einbinden von Elementen der Selbstregulation geschaffen. Selbstregulation wurde im Unterricht direkt mit den behandelten Inhalten verknüpft (vgl. Tabelle 4.1). In der KG wurden die gleichen fachlichen Inhalte unterrichtet, allerdings stand mehr Zeit für deren Erarbeitung zur Verfügung. Zur Standardisierung des Unterrichts wurde auch für die Variante mit Selbstregulationsanregung jede Stunde detailliert in einem Stundenverlaufsplan festgehalten. Die beteiligten Lehrer(innen) erhielten Manuale, die neben weiteren Informationen zum Ablauf der Studie diese Stundenverlaufspläne enthielten. Deren Versionen unterschieden sich entsprechend zwischen TG und KG. Vor dem Beginn der Interventionsphase fanden mehrere 
Treffen statt, in denen die geplanten Unterrichtsstunden besprochen, diskutiert und Optimierungsvorschläge eingearbeitet wurden. Diese Treffen fanden für Lehrer(innen) der TG und KG getrennt statt, um zu vermeiden, dass Elemente der Selbstregulationsanregung für den Basis-Unterricht übernommen werden. 


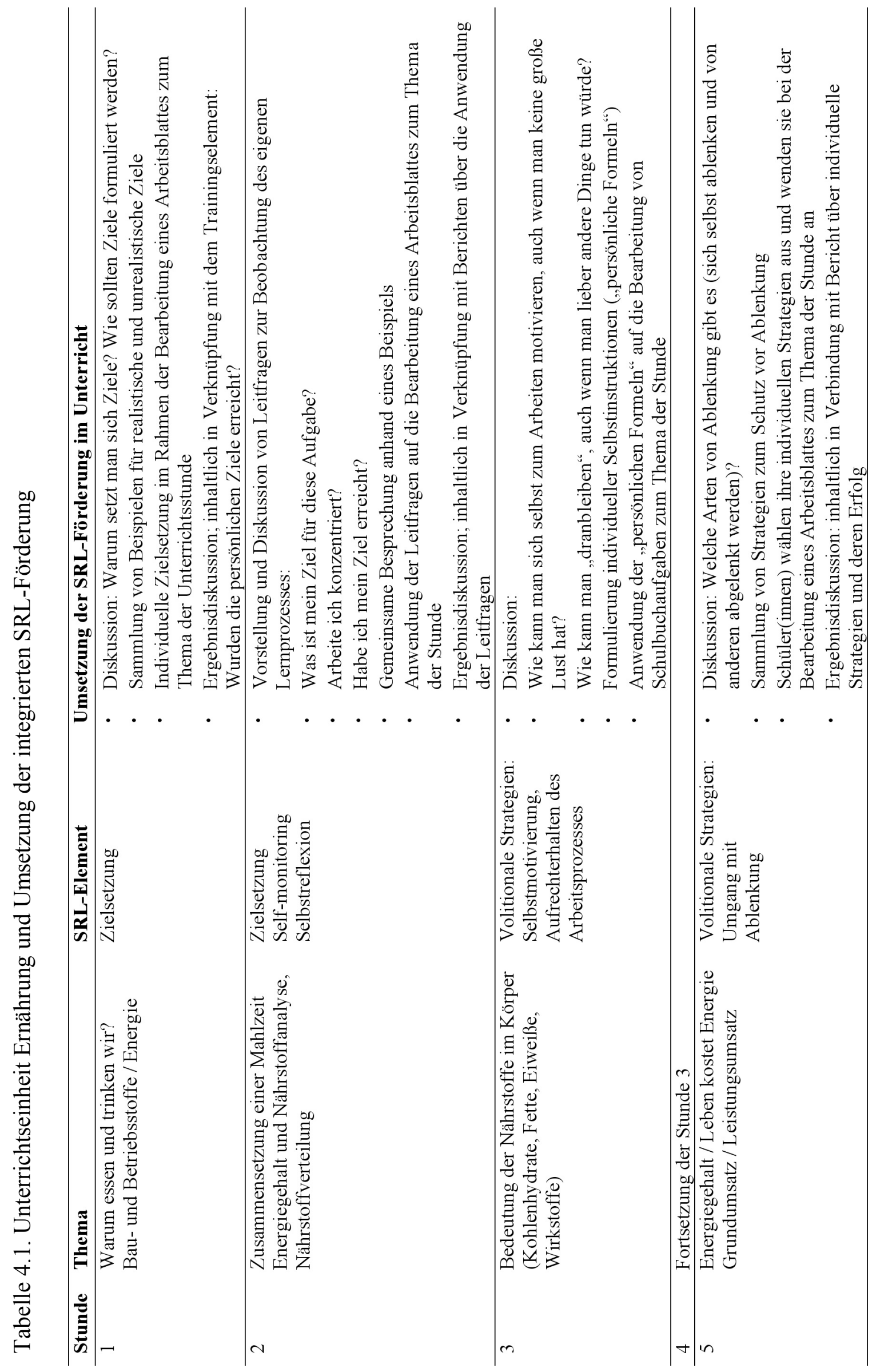




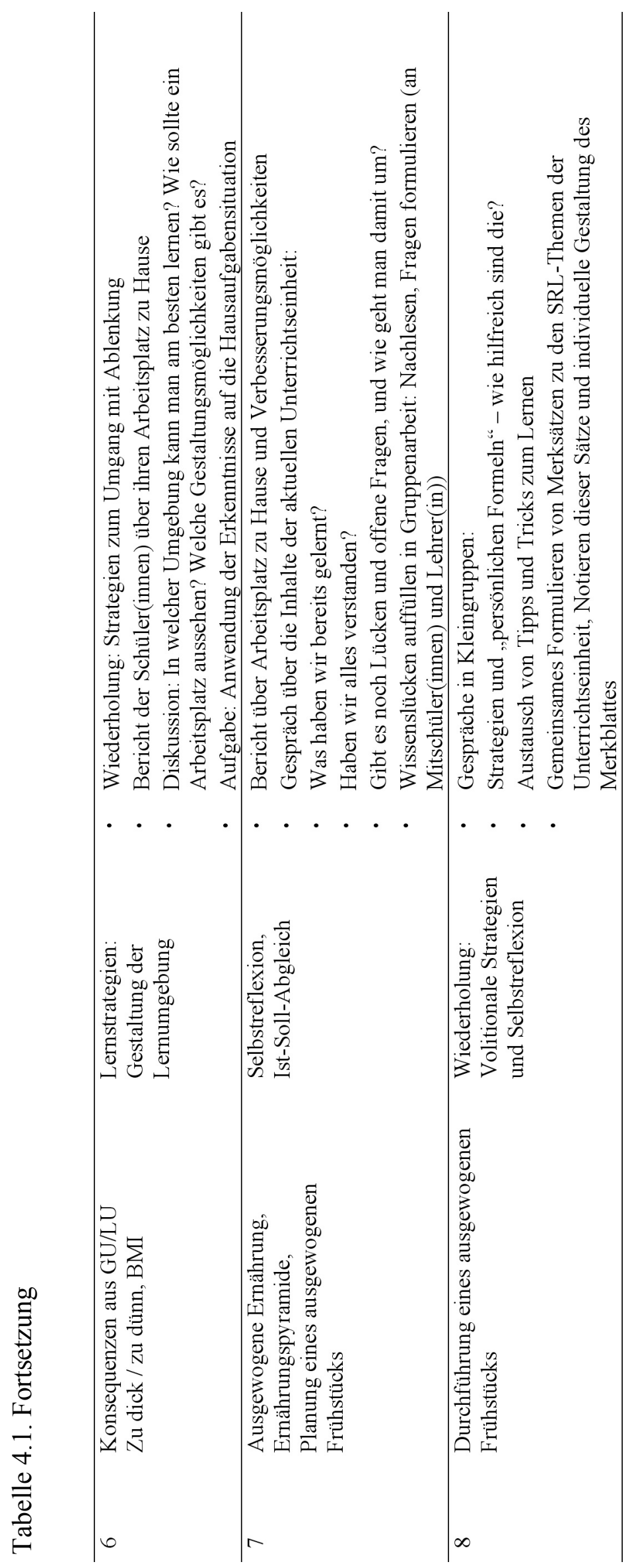


Ablauf

Die Untersuchung erstreckte sich über acht Wochen. Davon entfielen sechs Wochen auf die Unterrichtsintervention sowie Prätest und Posttest I, d.h. auf die Phase I. Die verbleibende Zeit wurde auf einen (in den Bildungsstandards vorgesehenen) Unterricht zum Kompetenzbereich Bewertung verwendet (Phase II). Die Unterrichtseinheit zum Thema Bewertung zielte auf die Vermittlung von Entscheidungsstrategien in umweltbezogenen Situationen. Ziel war die Förderung des systematischen Abwägens zwischen verschiedenen Wahlmöglichkeiten anhand von vorgegebenen Kriterien. Die Schüler(innen) lernten die Entscheidungsfindung am Beispiel einer alltäglichen Entscheidungssituation, dem "Apfelkauf". Die Inhalte dieser Unterrichtseinheit sind bei Bögeholz (2006) genauer beschrieben.

Vor der Realisierung des Unterrichts in Phase I wurde ein Prätest durchgeführt, um das Ausgangsniveau der Schüler(innen) hinsichtlich des ernährungsbezogenen Sachwissens und der Selbstregulation festzustellen. Der Durchführung der ersten Unterrichtseinheit folgte der Posttest I, bei dem sowohl ein Selbstregulationsfragebogen als auch ein lehrzielorientierter Test zu dem behandelten Thema Ernährung vorgelegt wurden. Posttest II wurde unmittelbar nach der Unterrichtseinheit der Phase II durchgeführt und diente der Ermittlung des Sachwissens, das die Schüler(innen) in der Unterrichtseinheit zum Kompetenzbereich Bewertung erworben hatten. Auf einen Prätest vor dieser Unterrichtseinheit wurde verzichtet, da kein nennenswertes Vorwissen zu diesem Bereich zu erwarten war.

\section{Messinstrumente}

\section{a) Selbstregulationsfragebogen}

Die Selbstregulationsfähigkeit wurde durch einen Fragebogen erfasst, der bereits im Rahmen der Evaluation eines Selbstregulationstrainings von Perels, Gürtler und Schmitz (2005) eingesetzt wurde (vgl. Anhang A). Auf zwei Subskalen („Extrinsische Motivation“ und „Umgang mit Fehlern“) wurde wegen der mangelnden Passung mit den Interventionsinhalten verzichtet. Die Bereiche selbstregulierten Lernens, die von dem Fragebogen erfasst werden und die sich gleichzeitig auf zentrale Inhalte der Intervention beziehen, sind „Ziele“, „Motivation / Volition“, „Lernstrategien“, „Selbstwirksamkeitserwartungen“ und „Selbstregulations-strategien“. Diese Skalen wurden zu einem Gesamtwert „Selbstregulation“ aggregiert. Tabelle 4.2 gibt eine Übersicht über die Skalen des für diese Studie angepassten Selbstregulationsfragebogens, Itembeispiele und die Reliabilitäten auf Basis der Daten von Perels, Gürtler und Schmitz (2005) bzw. der vorliegenden Studie. Alle Items wurden den Schü- 
ler(inne)n in Aussageform vorgelegt und waren auf einer vierstufigen Ratingskala $(1=$ stimmt gar nicht, 2 = stimmt eher nicht, 3 = stimmt eher, 4 = stimmt genau) zu beantworten.

Tabelle 4.2. Skalen des Selbstregulations-Fragebogens und Reliabilitäten in der Ursprungsstudie sowie in der vorliegenden Studie

\begin{tabular}{|c|c|c|c|c|}
\hline Abhängige Variablen & Anzahl Items $^{c}$ & Beispiel & $\alpha^{\mathbf{b}}$ & $\alpha^{\mathbf{c}}$ \\
\hline Ziele & 7 & $\begin{array}{l}\text { „Ich kann sehr hartnäckig sein, wenn } \\
\text { ich etwas erreichen möchte.“ }\end{array}$ & 0.84 & 0.49 \\
\hline Motivation/Volition ${ }^{\mathrm{a}}$ & 32 & $\begin{array}{l}\text { „Ich versuche mich dazu zu bringen, } \\
\text { Hausaufgaben zu erledigen, auch wenn } \\
\text { ich keine Lust habe.“ }\end{array}$ & 0.91 & 0.89 \\
\hline Lernstrategien & 25 & $\begin{array}{l}\text { „Vor dem Lernen überlege ich mir, wie } \\
\text { ich am besten vorgehen kann.“ }\end{array}$ & 0.90 & 0.87 \\
\hline Selbstwirksamkeit & 12 & $\begin{array}{l}\text { „Die Lösung schwieriger Probleme } \\
\text { gelingt mir immer, wenn ich mich dar- } \\
\text { um bemühe. }\end{array}$ & 0.86 & 0.76 \\
\hline $\begin{array}{l}\text { Selbstregulations- } \\
\text { strategien }\end{array}$ & 9 & $\begin{array}{l}\text { „Wenn ich von einer Sache abgelenkt } \\
\text { werde, komme ich schnell wieder zum } \\
\text { Thema zurück.“ }\end{array}$ & - & 0.62 \\
\hline Gesamtskala SRL $^{\mathrm{a}}$ & 85 & Aggregation der angeführten Skalen & 0.88 & 0.94 \\
\hline
\end{tabular}

Anmerkungen.

${ }^{a}$ in dieser Skala wurden Änderungen des bei Perels, Gürtler und Schmitz (2005) eingesetzten Instruments vorgenommen

${ }^{\mathrm{b}}$ bei Perels, Gürtler und Schmitz (2005)

${ }^{c}$ in der vorliegenden Studie 


\section{b) Lehrzielorientierte Tests}

Zur Erfassung des Sachwissens wurden zu den behandelten Themen der beiden Unterrichtseinheiten lehrzielorientierte Tests entwickelt. Das Vorwissen zum Thema Ernährung wurde im Prätest durch einen Multiple Choice-Test ermittelt. Nach der Unterrichtseinheit in Phase I wurde das Sachwissen zum Thema Ernährung durch einen Lückentext erfasst (vgl. Tabelle 4.3), der das Verständnis für zentrale Konzepte der Unterrichtseinheit prüfte und umfangreicher angelegt war als der Vorwissenstest. Nach der zweiten Unterrichtsphase wurde den Schüler(innen) ein Multiple Choice-Test vorgelegt. Dieser erfasste das Wissen, welches in der Unterrichtseinheit über Bewertung vermittelt wurde („Bewertungsstrukturwissen“, in Anlehnung an Bögeholz, 2006) (vgl. Tabelle 4.3). Dabei ging es vor allem um die Fähigkeit, in (umweltbezogenen) Alltagssituationen systematisch Entscheidungen zu treffen.

Tabelle 4.3. Itembeispiele für die lehrzielorientierten Tests

\begin{tabular}{|c|c|}
\hline Thema & Itembeispiel \\
\hline $\begin{array}{l}\text { Vorwissen } \\
\text { Ernährung } \\
\text { (Prätest) }\end{array}$ & $\begin{array}{l}\text { Was passiert im Körper, wenn man mehr Energie in Form von Nahrung zu sich } \\
\text { nimmt als man verbraucht? } \\
\text { a) Das Zuviel wird in Form von Fett gespeichert. } \\
\text { b) Man kann sich besser konzentrieren und bessere Leistungen erbringen. } \\
\text { c) Die Sehfähigkeit verschlechtert sich. } \\
\text { d) Es werden hauptsächlich Muskeln aufgebaut }\end{array}$ \\
\hline $\begin{array}{l}\text { Ernährung } \\
\text { (Posttest I) }\end{array}$ & 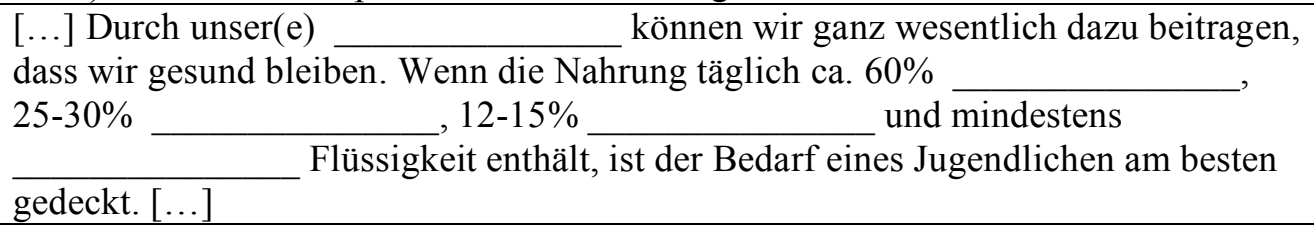 \\
\hline $\begin{array}{l}\text { Bewertung } \\
\text { (Posttest II) }\end{array}$ & $\begin{array}{l}\text { Bei welcher Aussage handelt es sich um eine Gewichtung? } \\
\text { a) Ich mag am liebsten süße Äpfel und deshalb bekommen alle süßen Äpfel die } \\
\text { meisten Punkte. } \\
\text { b) Ich nehme den Apfel „Boskop“, weil er die meisten Punkte bekommen hat. } \\
\text { c) Der Geschmack ist mir bei einem Apfel zwei Mal so wichtig wie der Preis. } \\
\text { d) Ich bringe die Apfelsorten je nach erreichter Punktzahl in eine Rangfolge. } \\
\text { Warum sollte man für eine Bewertung erstmal Kriterien festlegen, die einem wichtig } \\
\text { sind? } \\
\text { a) damit man nicht falsch rechnet } \\
\text { b) weil man sich so die eigenen Vorlieben klar machen kann } \\
\text { c) damit man mehr Kriterien hat als die anderen aus der Klasse } \\
\text { d) weil man sich dann auch falsch entscheiden darf }\end{array}$ \\
\hline
\end{tabular}




\subsubsection{Ergebnisse}

\section{Unterrichtsintegrierte Förderung selbstregulierten Lernens}

Die Subskalen des Selbstregulationsfragebogens interkorrelierten signifikant in einem Bereich von 0.31 bis 0.62. Dieser Befund deutet darauf hin, dass es sich um durchaus voneinander unterscheidbare Aspekte selbstregulierten Lernens handelt, die jedoch in einer Höhe zusammenhängen, die auch die Bildung einer Gesamtskala rechtfertigt.

Tabelle 4.4 enthält die Mittelwerte und Standardabweichungen der Selbstregulationsskalen getrennt nach Trainings- und Kontrollgruppe für Prätest und Posttest. Im Prätest zeigten sich keine signifikanten Gruppenunterschiede auf den Subskalen (Ziele: $F_{(1,183)}<1$; Motivation: $F_{(1,183)}<1$; Lernstrategien: $F_{(1,183)}<1$; Selbstwirksamkeit: $F_{(1,183)}<1, p=0.536$; Selbstregulationsstrategien: $\left.F_{(1,183)}=2.469, p=0.116\right)$ sowie der Gesamtskala SRL $\left(F_{(1,183)}=1.421\right.$, $p=0.235)$. Zur Überprüfung der Wirkung der Unterrichtseinheit mit Selbstregulationsförderung wurden für die abhängigen Variablen 2 (Gruppen) x 2 (Messzeitpunkte)-faktorielle Messwiederholungsvarianzanalysen durchgeführt. Die Ergebnisse für die Subskalen sowie die Gesamtskala werden im Folgenden berichtet. Abbildung 4.1 veranschaulicht die Ergebnisse für diejenigen Skalen, auf denen tendenzielle oder signifikante Effekte gefunden wurden.

Ziele. Es wurden keine Haupteffekte gefunden. Es zeigte sich ein tendenzieller Interaktionseffekt zwischen Gruppe und Messzeitpunkt $\left(F_{(1,183)}=3.696, p=0.056\right.$, eta $\left.{ }^{2}=0.020\right)$. Wie Abbildung $4.1 \mathrm{zu}$ entnehmen ist, geht dieser tendenzielle Effekt jedoch auf eine unerwartete Verschlechterung in der Kontrollgruppe zurück.

Motivation. Für diese Skala resultierte ein signifikanter Haupteffekt des Messzeitpunktes $\left(F_{(1,183)}=8.887, p=0.003\right.$, eta $\left.^{2}=0.046\right)$ sowie der Gruppe $\left(F_{(1,183)}=3.981\right.$, $p=0.047$ eta $\left.^{2}=0.021\right)$. Es wurde keine Interaktion nachgewiesen.

Lernstrategien. Hier zeigten sich keine Haupteffekte für Gruppe und Messzeitpunkt, aber eine signifikante Interaktion zwischen diesen Faktoren $\left(F_{(1,183)}=4.016, p=0.047\right.$, $\left.\operatorname{eta}^{2}=0.021\right)$. Post-hoc-Analysen ergaben einen signifikanten Anstieg für die Trainingsgruppe $\left(t_{(106)}=-2.639, p=0.010, r=0.248\right)$, nicht aber für die Kontrollgruppe $\left(t_{(77)}=0.321\right.$, $p=0.749, r=0.037)$.

Selbstwirksamkeit. Es zeigten sich ein signifikanter Haupteffekt des Messzeitpunktes $\left(F_{(1,183)}=6.051, p=0.015\right.$, eta $\left.^{2}=0.032\right)$ und eine tendenziell signifikante Interaktion $\left(F_{(1,183)}=3.224, p=0.074\right.$, eta $\left.^{2}=0.017\right)$. 
Selbstregulationsstrategien. Für diese Skala ergab die Varianzanalyse lediglich einen signifikanten Haupteffekt für die Gruppe $\left(F_{(1,183)}=4.830, p=0.029\right.$, eta $\left.^{2}=0.026\right)$. Es wurde keine Interaktion gefunden.

Gesamtskala SRL. Es zeigten sich ein signifikanter Haupteffekt des Messzeitpunktes $\left(F_{(1,183)}=6.606, p=0.011\right.$, eta $\left.^{2}=0.035\right)$, ein tendenziell signifikanter Haupteffekt der Gruppe $\left(F_{(1,183)}=3.816, p=0.052\right.$, eta $\left.^{2}=0.020\right)$, sowie eine signifikante Interaktion $\left(F_{(1,183)}=3.992, p=0.047\right.$, eta $\left.{ }^{2}=0.021\right)$. Post-hoc-Analysen ergaben einen signifikanten Anstieg für die Trainingsgruppe $\left(t_{(106)}=-3.492, p=0.001, r=0.321\right)$, nicht aber für die Kontrollgruppe $\left(t_{(77)}=-0.380, p=0.705, r=0.043\right)$.

Der tendenziell signifikante Interaktionseffekt für die Skala Selbstwirksamkeit sowie die signifikanten Interaktionseffekte für die Skala Lernstrategien und die Gesamtskala SRL unterstützen insgesamt unsere Erwartung, dass selbstreguliertes Lernen unterrichtsintegriert gefördert werden kann.

Tabelle 4.4. Mittelwerte (und Standardabweichungen) der Selbstregulationsskalen für Trainings- und Kontrollgruppe

\begin{tabular}{|c|c|c|c|c|c|}
\hline \multirow[t]{2}{*}{ Abhängige Variablen } & & \multicolumn{2}{|c|}{$\begin{array}{l}\text { Trainingsgruppe } \\
\qquad(\mathrm{n}=107)\end{array}$} & \multicolumn{2}{|c|}{$\begin{array}{c}\text { Kontrollgruppe } \\
\quad(n=78)\end{array}$} \\
\hline & & $\mathrm{M}$ & (SD) & $\mathrm{M}$ & (SD) \\
\hline \multirow[t]{2}{*}{ Ziele } & Prätest & 3.00 & $(0.38)$ & 3.03 & $(0.43)$ \\
\hline & Posttest & 3.03 & $(0.41)$ & 2.93 & $(0.47)$ \\
\hline \multirow[t]{2}{*}{ Motivation } & Prätest & 2.83 & $(0.42)$ & 2.73 & $(0.42)$ \\
\hline & Posttest & 2.92 & $(0.44)$ & 2.78 & $(0.46)$ \\
\hline \multirow[t]{2}{*}{ Lernstrategien } & Prätest & 2.86 & $(0.42)$ & 2.82 & $(0.39)$ \\
\hline & Posttest & 2.95 & $(0.44)$ & 2.81 & $(0.51)$ \\
\hline \multirow[t]{2}{*}{ Selbstwirksamkeit } & Prätest & 2.82 & $(0.40)$ & 2.78 & $(0.44)$ \\
\hline & Posttest & 2.93 & $(0.40)$ & 2.80 & $(0.46)$ \\
\hline \multirow[t]{2}{*}{ Selbstregulation } & Prätest & 2.72 & $(0.43)$ & 2.61 & $(0.46)$ \\
\hline & Posttest & 2.81 & $(0.48)$ & 2.64 & $(0.55)$ \\
\hline \multirow[t]{2}{*}{ Gesamtskala SRL } & Prätest & 2.84 & $(0.34)$ & 2.78 & $(0.33)$ \\
\hline & Posttest & 2.93 & $(0.38)$ & 2.79 & $(0.40)$ \\
\hline
\end{tabular}



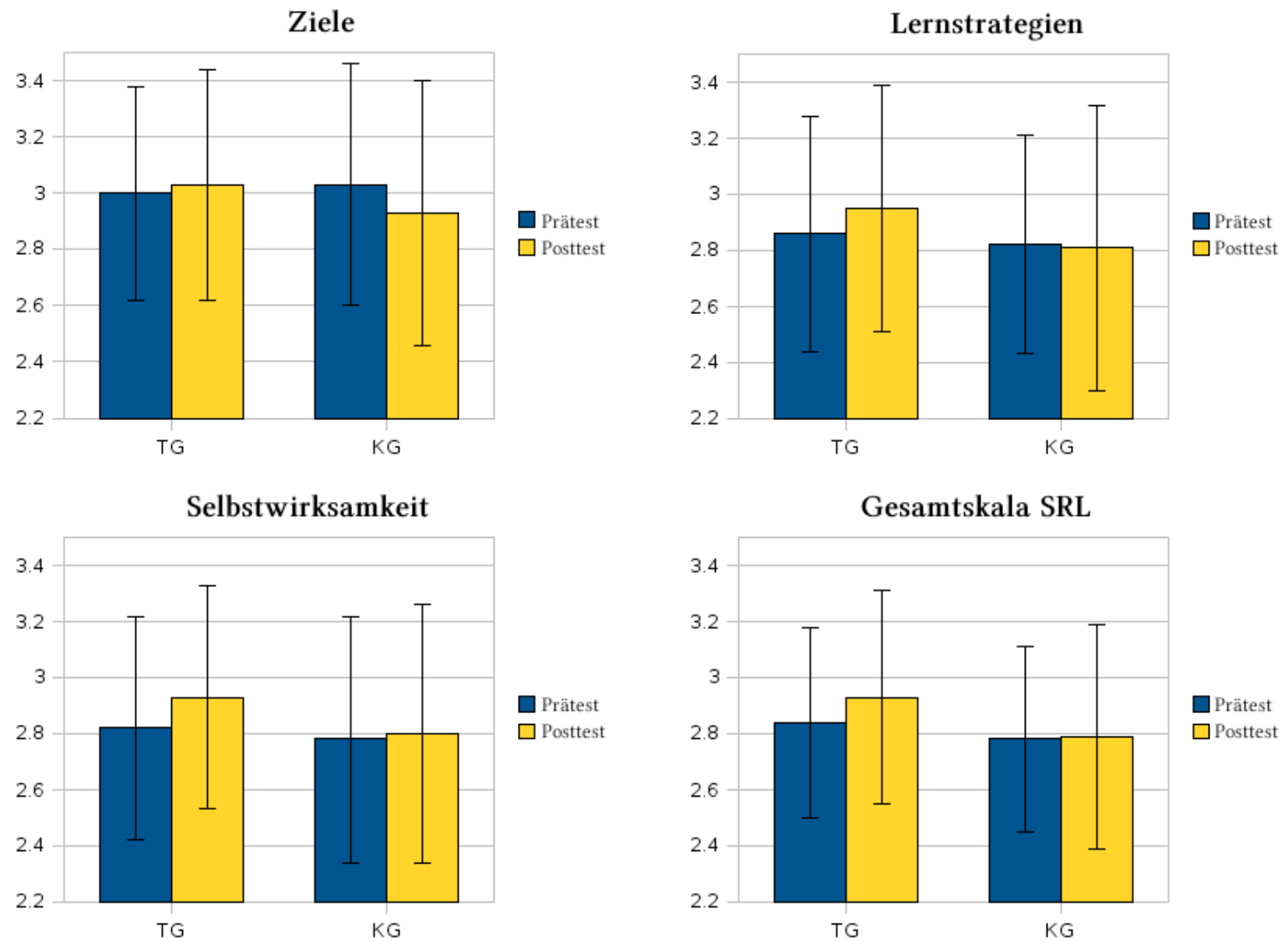

Abbildung 4.1. Interaktion Gruppe x Messzeitpunkt; Subskalen und Gesamtskala SRL

\section{Fachbezogenes Wissen}

Zur besseren Vergleichbarkeit der drei verschiedenen Tests zur Erfassung des fachbezogenen Wissens wurden die entsprechenden Rohwerte in z-Werte transformiert. Tabelle 4.5 zeigt die mittleren z-Werte sowie die Standardabweichungen für die drei Tests. Zum ersten Messzeitpunkt ergab die einfaktorielle Varianzanalyse keine Unterschiede zwischen Trainings- und Kontrollgruppe hinsichtlich des Vorwissens zum Thema Ernährung $\left(F_{(1,183)}<1\right)$. Im Posttest I resultierte ebenfalls kein Unterschied im ernährungsbezogenen Wissen $\left(F_{(1,183)}<1\right)$. Dieses Resultat bestätigt, dass das fachbezogene Lernergebnis in der Phase der unterrichtsintegrierten Selbstregulationsförderung nicht schlechter ausfällt. 
Tabelle 4.5. Mittlere z-Werte (und Standardabweichungen) der fachbezogenen Tests für Trainings- und Kontrollgruppe.

\begin{tabular}{lllllll}
\hline Abhängige Variable & & \multicolumn{2}{c}{ Trainingsgruppe } & & \multicolumn{2}{c}{ Kontrollgruppe } \\
\cline { 3 - 3 } \cline { 7 - 8 } & & z-Wert & $(\mathrm{SD})$ & & z-Wert & (SD) \\
\hline Vorwissen Ernährung & Prätest & 0.05 & 1.00 & & -0.03 & 0.94 \\
Sachwissen Ernährung & Posttest I & -0.03 & 0.99 & & 0.04 & 1.02 \\
\hline Sachwissen Bewertung & Posttest II & 0.15 & 1.04 & & -0.13 & 0.91 \\
\hline
\end{tabular}

\section{Leistungsentwicklung nach der Intervention}

Um zu prüfen, ob die Selbstregulationsanregung zu positiven Effekten auf das Lernvermögen in einem neuen Inhaltsbereich führt, wurde im Posttest II das in der zweiten Unterrichtseinheit vermittelte bewertungsbezogene Wissen gemessen (vgl. Tabelle 4.5). In der einfaktoriellen Varianzanalyse zeigte sich ein tendenziell signifikanter Effekt zugunsten der Trainingsgruppe $\left(F_{(1,170)}=3.432 ; p=0.066\right.$, eta $\left.{ }^{2}=0.020\right)$. Bei einseitiger Testung fällt dieser hypothesenkonforme Effekt signifikant aus. Dieses Ergebnis weist auf eine positive Auswirkung der unterrichtsintegrierten Anregung zur Selbstregulation auf den Wissenserwerb in einem neuen Themengebiet hin.

\subsubsection{Diskussion}

In der vorliegenden Studie wurde die Wirksamkeit einer in den Unterricht integrierten Anregung zum selbstregulierten Lernen untersucht. In einem ersten Schritt ging es dabei um die Frage, ob eine Unterrichtsvariante mit Selbstregulationsanregung spezifisch wirksam ist und zu einer Verbesserung der Selbstregulation führt.

Die Resultate des Prätest-Posttest-Vergleichs deuten insgesamt auf eine positive Wirkung der Unterrichtsintervention hin. Der signifikante Interaktionseffekt auf der Gesamtskala spricht für einen Anstieg der Selbstregulation bei den Schüler(inne)n in der Trainingsgruppe. Der tendenzielle Interaktionseffekt auf der Skala Selbstwirksamkeit sowie der signifikante Interaktionseffekt auf der Skala Lernstrategien weisen darauf hin, dass in diesen Bereichen ein Aufbau von Selbstregulation angestoßen wurde. Dennoch bleiben die Resultate leicht hinter unseren Erwartungen zurück: Die Effektstärken sind durchweg klein, und auf den Skalen Motivation und Selbstregulationsstrategien konnten nicht einmal kleine Effekte nachgewiesen 
werden. Eine Erklärung könnte in der relativ kurzen Intervention liegen: In acht Unterrichtsstunden entfielen lediglich jeweils ca. 15 Minuten auf Selbstregulationsinhalte. Möglicherweise hat diese begrenzte Zeit nicht ausgereicht, um die intendierten Inhalte mit der nötigen Intensität zu thematisieren. Besonders hinsichtlich der nicht festgestellten Veränderung auf der Skala Motivation vermuten wir, dass die Interventionszeit für eine positive Veränderung zu knapp bemessen war. So finden z.B. Schreblowski und Hasselhorn (2001) in einer explizit als Motivänderungsmaßnahme deklarierten Intervention nur sehr bedingt positive Veränderungen. Sie begründen dies damit, dass für substanzielle Motivänderungen bei Jugendlichen mehr Zeit veranschlagt werden müsse, was wir auch im Bezug auf die vorliegende Studie für nachvollziehbar halten. Der tendenzielle Effekt auf der Skala Ziele geht lediglich auf eine Verschlechterung der Kontrollgruppe zurück, kann also nicht als erwartungsgemäße Verbesserung der Trainingsgruppe gewertet werden. Das Resultat deutet ähnlich wie bei Perels, Gürtler und Schmitz (2005) darauf hin, dass die eher knappe Intervention für dieses Selbstregulationselement nicht ausreichend effektiv war. Zudem ist die Reliabilität dieser Skala in unserer Studie mit $\alpha=0.49$ nicht zufriedenstellend, so dass das Ergebnis ohnehin nur mit Vorbehalt interpretiert werden kann.

Eine weitere Beschränkung unserer Studie ist im Selbstaussageformat des Fragebogens zu sehen. Wir wählten diese Art der Messung aufgrund der ökonomischen Einsetzbarkeit (vgl. Spörer \& Brundstein, 2006), die besonders für die Durchführung von Studien im Schulkontext eine nicht zu unterschätzende Rolle spielt. Streng genommen kann jedoch auf diese Weise nicht das tatsächliche Regulationsverhalten gemessen werden, sondern lediglich die diesbezügliche Einschätzung der Schüler(innen) (vgl. Artelt, 1999, 2000). Artelt und Schellhas (1996) zeigen, dass nicht unbedingt von einem Zusammenhang zwischen dem selbst berichteten Einsatz von Lernstrategien und deren tatsächlicher Anwendung ausgegangen werden kann. Möglicherweise verfügen Schüler(innen) zwar über das nötige Strategiewissen (und berichten durchaus auch von der Nutzung), jedoch sind sie nicht in der Lage, die Strategien in entsprechenden Situationen auch adäquat einzusetzen (Hasselhorn, 1992, 1996). Wird in der Trainingsgruppe nach der Intervention von einem stärkeren Gebrauch von Strategien und erhöhter Selbstregulation berichtet, so kann das durchaus auch auf das bei diesen Schüler(inne)n erhöhte Bewusstsein für das Thema Strategien zurückgehen, jedoch nicht unbedingt auf deren häufigere Nutzung. Es ist möglich, dass das in der Intervention erworbene Wissen das Antwortverhalten beeinflusst hat. Darüber hinaus kann im retrospektiven Selbstbericht auch die Tendenz zu sozialer Erwünschtheit die Validität des Verfahrens gefährden. Vor diesem Hintergrund sind die durch die Fragebogenmessung erhaltenen Ergebnisse kri- 
tisch zu betrachten. Zukünftig wäre es wünschenswert, den Ansatz von Artelt (1999, 2000) aufzugreifen und die Messung durch handlungsnahe Analysen abzusichern.

Trotz der genannten Schwächen geben die Ergebnisse unseres Erachtens Anlass zu einer optimistischen Betrachtung des hier vorgestellten Ansatzes, selbstreguliertes Lernen in Verknüpfung mit den jeweiligen fachlichen Inhalten in den naturwissenschaftlichen Unterricht zu integrieren. In zukünftigen Studien zur unterrichtsintegrierten Förderung selbstregulierten Lernens könnte der Frage, wie die im Unterrichtskontext dafür zur Verfügung stehende Zeit noch effektiver genutzt werden kann, zusätzliche Aufmerksamkeit gewidmet werden. Unser Resultat steht im Einklang mit den Arbeiten von den Elzen-Rump und Leutner (2007) sowie Leopold, den Elzen-Rump und Leutner (2006), die ebenfalls Elemente selbstregulierten Lernens innerhalb des naturwissenschaftlichen Unterrichts förderten. Der von den $\mathrm{Au}-$ tor(inn)en realisierte Ansatz unterscheidet sich von dem der vorliegenden Studie jedoch hinsichtlich der Herangehensweise und der Auswahl der Trainingselemente. Wir entwickelten gemeinsam mit den Lehrkräften eine Unterrichtseinheit und bezogen in die Intervention eine große Bandbreite an Selbstregulationsfacetten ein. Es kann bezüglich dieser breit angelegten Auswahl an Trainingsinhalten argumentiert werden, dass ein solches Vorgehen zuungunsten einer fokussierten Förderung einzelner Strategien geht. Das von uns verfolgte Ziel war es jedoch, einen großen Teil des im oben beschriebenen Modell (Zimmerman, 2000) abgebildeten Selbstregulationsprozesses mit Trainingselementen abzudecken (vgl. Perels, Gürtler \& Schmitz, 2005). In zukünftigen Studien wäre zu ermitteln, welche dieser Komponenten sich besonders gut für die Integration in den Fachunterricht eignen und Effekte erzielen, so dass die Förderung straffer und fokussierter gestaltet werden könnte. Besonders im Hinblick auf die Vereinbarkeit von Fachunterricht und fächerübergreifender Lernförderung wäre das ein relevantes Ziel.

Unsere zweite Frage bezog sich auf die Auswirkungen der Selbstregulationsanregungen auf schulisches Lernen. Ein wichtiges Anliegen bestand darin zu gewährleisten, dass die Einbindung von Komponenten der Selbstregulationsförderung in den Unterricht nicht zu geringeren fachbezogenen Lernleistungen führt. Zu einem solchen negativen Nebeneffekt könnte es kommen, da a) weniger Zeit für fachliches Lernen zur Verfügung steht, b) der Neuheitswert der Selbstregulationselemente zu Lasten der Konzentration auf fachbezogene Inhalte gehen kann und c) in der Phase des Erwerbs neuer Strategien die Leistungen kurzfristig absinken können (Hasselhorn \& Gold, 2006, S. 97ff.). Die Resultate des lehrzielorientierten Tests im direkten Anschluss an die erste Unterrichtseinheit (Posttest I) zeigen jedoch, dass sich die Leistungen von Schüler(inne)n aus Trainings- und Kontrollgruppe nicht signifikant 
voneinander unterscheiden. Wir werten diesen Befund dahingehend, dass die Anregung zur Selbstregulation nicht zuviel Unterrichtszeit beansprucht, gut in den Fachunterricht integrierbar ist und nicht vom Erwerb des relevanten Fachwissens ablenkt. Es ist zu vermuten, dass Anregungen dieser Art zu einer zusätzlichen Strukturierung der Unterrichtsstunden und zu fokussierter Arbeitsweise seitens der Lehrer(innen) sowie der Schüler(innen) geführt hat, die den Zeiteinsatz zu kompensieren vermochten.

Mit dem Nachweis der insgesamt erfolgreichen Selbstregulationsförderung war die Voraussetzung dafür erfüllt, dass die Frage nach dem Effekt der Intervention auf die Lernleistung in einem neuen Themengebiet sinnvoll prüfbar ist. Wir verglichen daher die Leistungen der beiden Gruppen im lehrzielorientierten Test, der sich auf die Inhalte der zweiten Unterrichtseinheit bezog. Hier konnte ein Vorteil der Trainingsgruppe nachgewiesen werden. Nach Abschluss der Unterrichtsvariante mit Selbstregulationsanregung kam es bei den Schüler(inne)n offenbar zu einem erfolgreichen Lernverlauf. Wir bewerten die hier dargestellte Intervention insgesamt als erfolgreich, da gezeigt werden konnte, dass die unterrichtsintegrierte Förderung in enger Kooperation mit Lehrkräften zu realisieren ist, es nicht zu fachbezogenen Leistungseinbußen kommt und die trainierten Schüler(innen) auch beim Lernen in einem neuen Themengebiet von den erworbenen Lernkompetenzen profitieren. Unser Ergebnis unterstützt die aus der Studie von Souvignier und Antoniou (2007) hervorgehende Implikation, dass eine kooperative Arbeitsweise von Wissenschaftler(inne)n und Lehrer(inne)n ein wichtiges Kriterium für den Erfolg innovativer Unterrichtsansätze darstellt. Wenngleich dieser Aspekt keiner expliziten Testung unterzogen wurde, spiegelt er unsere positive Erfahrung wider. Diesem Punkt sollte in Zukunft verstärkt Bedeutung beigemessen werden. Es wäre wünschenswert, derartige Projekte einer wissenschaftlichen Evaluation zugänglich zu machen. In diesem Zuge erachten wir es weiterführend als sinnvoll zu untersuchen, ob differentielle Trainingseffekte entstehen. Eine Förderung, die in der Schule ansetzt, sollte gewährleisten, dass alle Teilnehmenden potenziell profitieren können und kein „Matthäuseffekt“ verursacht wird. Dieser in der pädagogisch-psychologischen Interventionsforschung häufig zu findende Effekt geht darauf zurück, dass die ohnehin leistungsstarken Schüler(innen) mehr von den Fördermaßnahmen profitieren als die als die eher benachteiligten. Ein solcher ungünstiger Nebeneffekt kann zum Beispiel aufgrund des hohen Sprachanteils des Programms oder unterschiedlicher motivationaler Voraussetzungen der Lernenden auftreten.

Die aus unserer Studie abzuleitenden Aussagen unterliegen selbstverständlich neben den bereits thematisierten methodischen einer Reihe von generellen Beschränkungen. So fand die Intervention in nur einer Schule statt, so dass die positiven Effekte zum Teil auch auf das 
gute Arbeitsklima, die Aufgeschlossenheit der Lehrer(innen) und die Unterstützung durch die Schulleitung zurückgeführt werden können. Dies sind vergleichsweise ideale Umstände, was aber auch zeigt, dass unser Ansatz beim Vorliegen guter Rahmenbedingungen erfolgreich realisiert werden kann. Darüber hinaus war es aus praktischen Gründen und unter Berücksichtigung schulischer Alltagsbedingungen nicht möglich eine randomisierte Gruppenzuweisung vorzunehmen. Da wir jedoch zeigen konnten, dass sich die Gruppen nicht hinsichtlich ihres Ausgangsniveaus in den untersuchten abhängigen Variablen unterschieden, halten wir dies für akzeptabel. Für ein solches Vorgehen spricht, dass die Intervention ohne jegliche Veränderung der Zusammensetzung der Klassen in den Schulalltag eingebettet werden konnte. Die hier vorgestellte Studie ist für den Ansatz einer unterrichtsintegrierten Forschung im Bereich von Selbstregulation, Lernstrategien und Motivation ermutigend. Eine Weiterentwicklung unter Einbeziehung von für die Unterrichtsgestaltung relevanten Fragen erachten wir als vielversprechend. 


\subsection{Herausforderungen für Trainingsansätze im Schulkontext}

Die Wirksamkeit der in Kapitel 4.1 vorgestellten unterrichtsintegrierten Förderung selbstregulierten Lernens wurde in drei Schritten belegt (Labuhn, Bögeholz und Hasselhorn, 2008): Die Untersuchung liefert den statistisch abgesichterten Nachweis 1.) des Trainingseffektes auf die Selbstregulation, festgestellt im direkten Anschluss an die Intervention, 2.) des vergleichbaren fachbezogenen Lernergebnisses in Trainings- und Kontrollgruppe in der Phase der unterrichtsintegrierten Selbstregulationsförderung und 3.) des Lernvorteils der Trainingsgruppe in einer anschließenden neuen und von der Intervention unabhängigen Unterrichtseinheit. Zwei weitere wichtige Belege für den Erfolg von Trainingsansätzen im Schulkontext stehen im Zusammenhang mit der dargestellten Studie noch aus und werden im vorliegenden Teilkapitel sowie in dem in Kapitel 4.3 vorgestellten empirischen Beitrag aufgegriffen:

1. Langfristige Wirksamkeit: Der praktische Nutzen unterrichtsintegrierter Ansätze ist erst dann wirklich gegeben, wenn nicht nur kurzfristige Effekte erzielt werden, sondern Selbstregulationsfähigkeit und Lernerfolg dauerhaft gesteigert werden können.

2. Gewährleistung der Chancengleichheit: Förderung, die in der Schule ansetzt, sollte gewährleisten, dass alle Teilnehmenden potenziell profitieren können und kein „Matthäuseffekt" verursacht wird. Unter Matthäuseffekt versteht man das in der pädagogischpsychologischen Interventionsforschung häufig zu findende Ergebnismuster, dass die ohnehin leistungsstarken Schüler(innen) mehr von den Fördermaßnahmen profitieren als die eher benachteiligten.

\section{Langfristige Wirksamkeit der Selbstregulationsförderung}

Zimmerman (1990) betont, dass langfristige Interventionseffekte nur $\mathrm{zu}$ erwarten seien, wenn die Förderung sowohl metakognitive als auch motivationale und behaviorale Komponenten der Selbstregulation berücksichtigt. Entsprechend dem Prinzip des reziproken Determinismus hat auch die Lernumwelt einen entscheidenden Einfluss darauf, ob einmal erworbenes Verhalten weiterhin gezeigt wird. Lernsituationen, die weniger stark strukturiert sind als der Kontext, in dem das Training stattgefunden hat, wirken häufig einer Aufrechterhaltung entgegen (Pajares, 2008). Mangelnde Unterstützung, z.B. in Form positiven Feedbacks, sowie fehlende Anwendungsgelegenheiten können dazu führen, dass in einem kurzfristig effektiven Training erworbene Selbstregulationsfähigkeiten auf längere Sicht nicht mehr umgesetzt und als Folge möglicherweise wieder aus dem Verhaltensrepertoire gelöscht wer- 
den. Darüber hinaus spielen gemäß der sozial-kognitiven Perspektive soziale Einflüsse eine wichtige Rolle: Werden Inhalte des Trainings, wie z.B. Lern- und Selbstregulationsstrategien auch von anderen Schüler(inne)n angewandt und positiv bewertet, steigt die Wahrscheinlichkeit, dass sich ein solches Verhalten über einen längeren Zeitraum etabliert. Lernende dienen sich im günstigsten Falle gegenseitig als Verhaltensmodelle. Ohne die explizite Unterstützung durch Lehrkräfte bedarf die Aufrechterhaltung des durch das Training gerade neu aufgebauten Verhaltens jedoch ausgeprägter Selbstregulationsfähigkeiten entsprechend den Niveaus der Selbstkontrolle oder Selbstregulation nach Zimmerman (2000).

Von den in Kapitel 3.2 erwähnten Trainings zur unterrichtsintegrierten Förderung selbstregulierten Lernens berichten Glaser und Brunstein (2007a, 2007b) sowie Souvignier und Mokleshgerami (2006) von zeitlich stabilen Effekten. Diese Resultate entsprechen der theoriebasierten Erwartung insofern, als dass in den Programmen die kombinierte Förderung metakognitiver, motivationaler und behavioraler Komponenten realisiert wird, auf deren Bedeutung Zimmerman (1990) hinweist. Im Hinblick auf langfristige Trainingseffekte auf die fachbezogene Leistung berichten Leopold, den Elzen-Rump und Leutner (2006) für die Förderung des selbstregulierten Einsatzes von Lern- und Textbearbeitungsstrategien im Rahmen des naturwissenschaftlichen Unterrichts (mit vorangehendem computerbasierten Training) ein bemerkenswertes Resultat: Der Unterschied zwischen Trainings- und Kontrollgruppe war im Posttest (Textverständnistest) im direkten Anschluss an das Training deutlich geringer $(d=0.07)$ als drei Monate später $(d=0.29)$. Im Vergleich zur Kontrollgruppe zeigten trainierte Schüler(innen) zwar zunächst nur geringfügig bessere Leistungen, nach drei Monaten jedoch eine deutlich bessere Behaltensleistung.

Die Evaluation von Traningsprogrammen im Schulkontext sollte aus zwei Gründen auch eine langfristige Überprüfung der Effekte beinhalten: Zum einen, weil durch den Nachweis stabiler Interventionseffekte, insbesondere auf fachbezogene Leistungen, der Nutzen der Programme deutlich steigt und dadurch die Forderung nach einer Integration in den Schulalltag empirisch untermauert wird. Zum anderen weisen Resultate wie das von Leopold, den Elzen-Rump und Leutner (2006) vorgelegte darauf hin, dass die Erhebung von Follow-upDaten zu wichtigen Erkenntnissen hinsichtlich der Wirkmechanismen selbstregulierten Lernens führen kann. Für die in Kapitel 4.1 der vorliegenden Arbeit vorgestellte unterrichtsintegrierte Förderung wird daher in Kapitel 4.3 die noch ausstehende Klärung der langfristigen Wirkung (über ein Schulhalbjahr) vorgenommen. 


\section{Gewährleistung der Chancengleichheit}

Die unterrichtsintegrierte Förderung selbstregulierten Lernens sollte so angelegt sein, dass alle Teilnehmenden profitieren und nicht bestimmte Subgruppen systematisch benachteiligt werden. Ein Matthäuseffekt sollte also nicht auftreten. Abbildung 4.2. zeigt ein hypothetisches Datenmuster als Beispiel für einen Matthäuseffekt: Obwohl der Mittelwert der Trainingsgruppe im Posttest über dem der Kontrollgruppe liegt (die Varianzanalyse hier also einen signifikanten Effekt anzeigen dürfte), geht dieser Trainingseffekt lediglich auf den signifikanten Anstieg in der Teilgruppe der Schüler(innen) zurück, die schon im Prätest über ausgeprägte Fähigkeiten verfügen.

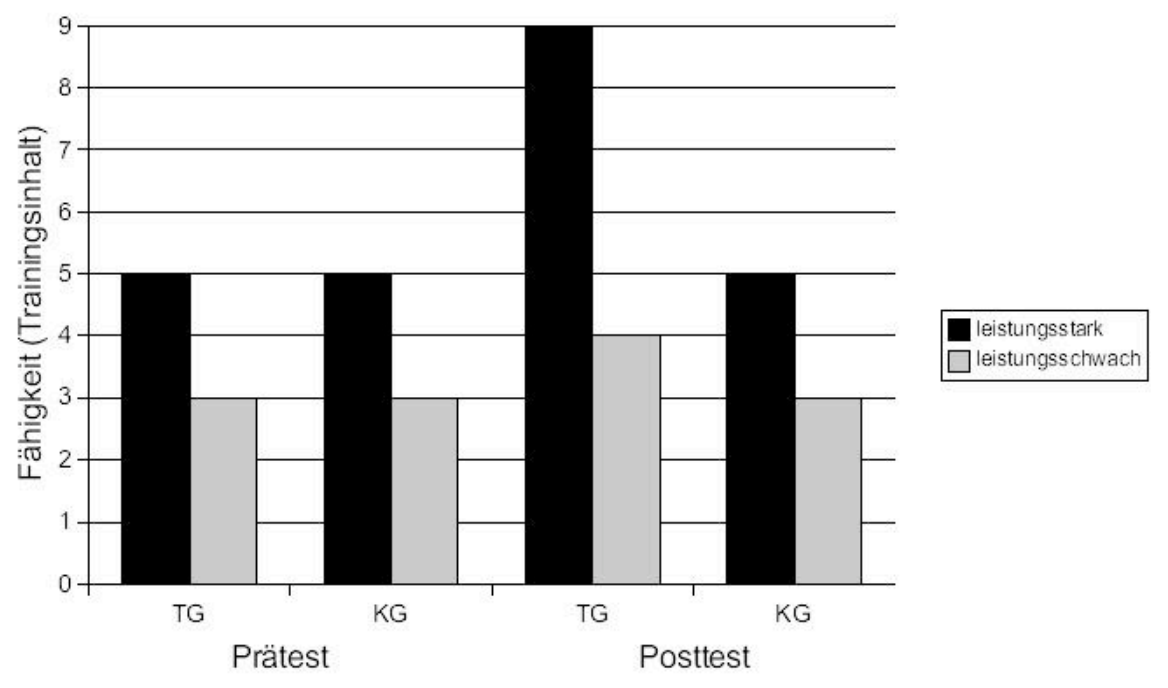

Abbildung 4.2. Beispiel für das Ergebnismuster eines Matthäuseffekts

Dieser nicht intendierte Nebeneffekt kann zum Beispiel aufgrund von Ausgangsunterschieden in den kognitiven Voraussetzungen oder in der Selbstregulationskompetenz der Lernenden auftreten, die sich differentiell auf die Verarbeitung der Trainingsinhalte auswirken. Zimmerman und Martinez-Pons (1990) berichten in einer vergleichenden Studie, dass leistungsstarke Schüler(innen) ausgeprägtere Selbstregulationsfähigkeiten und höhere Selbstwirkamkeitserwartungen zeigen als Schüler(innen) mit durchschnittlichem oder unterdurchschnittlichem Leistungsvermögen. Legt man die sozial-kognitive Auffassung selbstregulierten Lernens als eine Interaktion aus personen-, verhaltens- und umweltbezogenen Prozessen zugrunde, so zeigt die Analyse der Selbstregulationsstrategien der Schüler(inne)n, dass die leistungsstarken unter ihnen über adäquate Strategien zur Regulation aller drei Teilbereiche verfügen. Darüber hinaus sprechen die Ergebnisse der differentiellen Studie dafür, dass Selbstregulati- 
onsfähigkeiten und motivational günstige Selbstwirksamkeitserwartungen bei leistungsstarken Schüler(inne)n früher eine positive Entwicklung nehmen als bei Schüler(inne)n, die mittelmäßige oder unterdurchschnittliche Schulleistungen erbringen. Wie sich solche Leistungsund Selbstregulationsunterschiede jedoch auf die Wirkung einer Fördermaßnahme auswirken, bleibt noch zu klären.

Aufgrund des hohen Sprachanteils des unterrichtsintegrierten Förderansatzes könnte die Wirksamkeit der Selbstregulationsförderung auch zum Teil vom Migrationsstatus der Schüler(innen) abhängig sein. Jugendliche mit Migrationshintergrund kommen insgesamt betrachtet häufig aus Familien, die durch einen deutlich unterdurchschnittlichen sozioökonomischen Status gekennzeichnet sind und in denen kaum oder gar kein Deutsch gesprochen wird (Prenzel et al., 2007). Aus Resultaten von PISA 2000 (Baumert et al., 2001) geht die Annahme hervor, dass sich sprachliche Defizite kumulativ in Sachfächern auswirken. Sprachverständnisschwierigkeiten dürften damit den Lernprozess von Schüler(inne)n, die aufgrund ihrer kognitiven Kapazitäten eigentlich in der Lage wären, gute Leistungen zu zeigen, erheblich beeinträchtigen. Weiterhin sind Personen mit unzureichendem Leseverständnis in allen akademischen Bereichen in ihrem Kompetenzerwerb benachteiligt (Baumert et al., 2001). Eine Konsequenz, die sich u.a. aus der Problematik von sprachlichen Defiziten ergibt, zeigte sich in PISA 2006: Im Bereich der naturwissenschaftlichen Kompetenz schnitten in Deutschland Jugendliche mit Migrationshintergrund deutlich schlechter ab als Jugendliche ohne Migrationshintergrund (Prenzel et al., 2007). Die in Kapitel 4.1 vorgestellte unterrichtsintegrierte Selbstregulationsförderung wurde in einer integrierten Gesamtschule realisiert, in der die Klassen sehr heterogen aus Schüler(inne)n mit unterschiedlichen Lernvoraussetzungen, Schulleistungsniveaus und sozialen Hintergründen zusammengesetzt sind und der durchschnittliche Migrant(inn)enanteil ca. 40\% beträgt. Dieser Kontext unterstreicht die Relevanz der Überprüfung differentieller Trainingseffekte. Im folgenden Teilkapitel erfolgt daher über die langfristige Wirksamkeitsüberprüfung hinaus die empirische Klärung der Frage, ob alle Schüler(innen), unabhängig von ihren individuellen Voraussetzungen, von der Intervention profitieren. 


\subsection{Selbstregulationsförderung in einer Biologie-Unterrichtseinheit - langfristige und differentielle Wirksamkeit ${ }^{5}$}

\subsubsection{Zusammenfassung und Abstract}

Im vorliegenden Artikel werden langfristige und differentielle Effekte von unterrichtsintegrierter Selbstregulationsförderung in einer Biologie-Unterrichtseinheit im Fach Naturwissenschaften berichtet. Aufbauend auf einer Studie von Labuhn, Bögeholz und Hasselhorn (2008) mit 199 Schüler(inne)n der siebten Jahrgangsstufe wurde die langfristige Interventionswirkung auf die Selbstregulation und die fachbezogene Leistung untersucht. Es zeigte sich trotz eines generellen Absinkens der Selbstregulationswerte in den motivationalen Selbstregulationskomponenten ein stabiler Fördereffekt. Die im Follow-up gemessene fachbezogene Leistung der Trainingsgruppe lag deutlich über der Leistung der Vergleichsgruppe. Differentielle Analysen ergaben, dass Schüler(innen) mit unterschiedlichen Ausgangsvoraussetzungen im selbstregulierten Lernen gleichermaßen profitierten. Der deutliche langfristige Vorteil der Trainingsgruppe im fachbezogenen Test war jedoch nur für Schüler(innen) ohne Migrationshintergrund nachzuweisen.

\section{Abstract}

The present article reports further analyses of a previous project on self-regulated learning in science education (Labuhn, Bögeholz und Hasselhorn, 2008). The precedent study based on a lesson on nutrition that had been designed to improve seventh graders' $(N=199)$ self-regulation. The results of the here presented follow-up revealed that although selfregulation had slightly decreased, the experimental group still scored higher than the control group on motivational aspects of self-regulation and, furthermore, outperformed the control group on a knowledge test. Students with different levels of prerequisite skills in selfregulation benefited equally from the intervention. However, trained students from families with a migration background did not perform better on the follow-up knowledge test.

\footnotetext{
${ }^{5}$ Dieses Teilkapitel wurde zum Druck angenommen: Labuhn, A. S., Bögeholz, S. \& Hasselhorn, M. (in Druck). Selbstregulationsförderung in einer Biologie-Unterrichtseinheit - langfristige und differentielle Wirksamkeit. Zeitschrift für Entwicklungspsychologie und Pädagogische Psychologie.
} 
Die Kompetenz, neue Informationen kritisch zu prüfen und sie mit vorhandenen Kenntnissen zu verknüpfen, erfordert ein hohes Maß an Flexibilität und die Fähigkeit, Lernprozesse selbst zu initiieren und zu regulieren. Zimmerman (2002) sieht im Aufbau entsprechender individueller Kompetenzen eine zentrale Funktion von Bildung und Erziehung: Lernende sollten darin unterstützt werden, ein Bewusstsein für die eigenen Fähigkeiten und deren Weiterentwicklung aufzubauen. Schüler(innen) zum selbstregulierten Lernen zu befähigen, ist inzwischen Nationen übergreifend ein explizites Bildungsziel (OECD, 2004). Dies schlägt sich auch in den im Rahmen von PISA durchgeführten Lehrplananalysen nieder (Artelt, Baumert \& Julius-McElvany, 2003). Die Realität sieht jedoch häufig anders aus als aktuelle normative Referenzwerke es fordern: Der Vermittlung und Reproduktion von Faktenwissen wird häufig mehr Zeit eingeräumt als problemorientiertem und verständnisintensivem Lernen (Klieme \& Stanat, 2002). Letzteres dürfte nicht nur, aber auch besonders für die mathematisch-naturwissenschaftlichen Fächer von hoher Relevanz sein. Dass das Vermögen, den eigenen Lernprozess zu regulieren einen entscheidenden Faktor erfolgreichen Lernens darstellt, unterstreichen die inzwischen zahlreichen Studien, die die Relevanz von Kompetenzen aufzeigen, die über reines Faktenwissen hinausgehen (z.B. Zimmerman \& Martinez-Pons, 1986, 1988).

\section{Selbstregulation als Prozess}

Selbstreguliertes Lernen wird weitgehend übereinstimmend als ein dynamisches $\mathrm{Zu}$ sammenwirken von kognitiven, metakognitiven und motivationalen Aspekten des Lernens verstanden und meist als ein Prozess dargestellt (z.B. Boekaerts, 1999; Butler \& Winne, 1995; Pintrich, 2000; Schmitz, 2001; Zimmerman, 2000, 2002). Zimmerman (2000, 2002) beschreibt die Selbstregulation als einen individuellen Prozess, in dessen Rahmen der oder die Lernende zyklisch drei Phasen durchläuft: Vorbereitungsphase, Handlungsphase und Selbstreflexionsphase. Diese sozial-kognitive Auffassung, die nicht auf der Annahme einer statischen mentalen Fähigkeit oder akademischen Leistungskomponente aufbaut, bietet Ansatzpunkte für Förderung und Intervention an verschiedenen Punkten des Zyklus.

Die Vorbereitungsphase umfasst jene Prozesse, die vor der Bearbeitung einer Aufgabe ablaufen. Situationsbezogene motivationale Voraussetzungen wie z.B. Selbstwirksamkeitserwartungen spielen hier ebenso wie Zielsetzung und Planung eine wichtige Rolle für den weiteren Verlauf des Lernens. In der sich anschließenden Handlungsphase sind alle auf die Aufgabenbearbeitung bezogenen Prozesse von Bedeutung. Entscheidend für den Erfolg des Lernens sind hier (lern)strategisches Vorgehen sowie die Fähigkeit zum sogenannten Self- 
monitoring, d.h. der kritischen Beobachtung der eigenen, auf die Bearbeitung bezogenen Handlungen. Darüber hinaus spielen volitionale Strategien eine wichtige Rolle. Erfolgreich Lernende müssen sowohl in der Lage sein, ihre Aufmerksamkeit der Aufgabenbearbeitung zu widmen, als auch sie über einen längeren Zeitraum aufrecht zu erhalten und gegen Ablenkungen oder alternative/ konkurrierende Tätigkeiten (z.B. Freizeit oder der Kommunikation mit Mitschüler(inne)n) abzuschirmen. Der Handlungs- folgt die Selbstreflexionsphase, die sich inhaltlich direkt auf das Resultat der Aufgabenausführung bezieht. Die Reflexion über das erzielte Ergebnis umfasst einen Abgleich mit der persönlichen Zielsetzung sowie die Bewertung der eigenen Leistung in Verbindung mit Rückschlüssen auf die Arbeitsweise. Der Verlauf dieser Phase nimmt wiederum Einfluss auf den folgenden Selbstregulationszyklus. Je nach vorangegangenem Resultat beginnt die lernende Person die nächste Vorbereitungsphase beispielsweise mit entsprechenden Selbstwirksamkeitserwartungen, modifizierten persönlichen Zielen oder sie verändert ihre Bearbeitungsstrategien.

\section{Selbstregulationsförderung im Schulkontext.}

Es liegen eine Reihe von Forschungsarbeiten vor, die einen positiven Zusammenhang des selbstregulierten Lernens mit schulischen Leistungen aufzeigen (z.B. Zimmerman \& Martinez-Pons, 1986; 1988) und die darlegen, dass selbstreguliertes Lernen durch eine Intervention gefördert werden kann (vgl. Schunk \& Ertmer, 2000; Schunk \& Zimmerman, 1998). In den vergangenen Jahren wurden zunehmend Trainingsprogramme entwickelt, die eine Selbstregulationsförderung mit curricular validen Inhalten verknüpften und kontextspezifisch zu fördern intendierten. Dieser Ansatz stellte sich wiederholt als erfolgreich heraus (z.B. den Elzen-Rump \& Leutner, 2007; Glaser \& Brunstein, 2007; Leopold, den Elzen-Rump \& Leutner, 2006; Souvignier \& Mokhlesgerami, 2006 in einer schulischen Umsetzung des Trainingsansatzes von Schreblowski \& Hasselhorn, 2001). Für das Fach Mathematik entwickelten Perels, Gürtler und Schmitz (2005) ein prozessorientiertes Training, welches erfolgreich den gesamten Zyklus der Selbstregulation mit mathematischem Problemlösen kombiniert. Dieses Training fand allerdings außerhalb des Unterrichts statt. Fuchs et al. (2003) demonstrieren, dass sich auch im regulären Unterricht die Förderung selbstregulierten Lernens mit der Vermittlung von mathematischen Strategien verbinden lässt und die Leistung positiv beeinflusst wird. Die Effektivität dieses unterrichtsintegrierten Ansatzes zeigen auch Perels, Dignath und

Schmitz (in Druck) im Rahmen ihrer Interventionsstudie mit Sechstklässler(inne)n für den Mathematikunterricht auf. Die zahlreichen Hinweise auf eine positive Wirkung der Verknüpfung von selbstreguliertem und fachlichem Lernen sprechen für die Bedeutung weiterer For- 
schung zur systematischen Einbindung von Selbstregulation in den Fachunterricht. Die Metaanalyse von Souvignier und Antoniou (2007) zeigt auf, dass die Wirksamkeit von innovativen Unterrichtsansätzen im Schulalltag besonders gut ausfällt, wenn die Implementation in enger Kooperation zwischen Lehrer(inne)n und Wissenschaftler(inne)n vorgenommen wird. Ein Beispiel für eine erfolgreiche Umsetzung einer entsprechenden Implementation im naturwissenschaftlichen Unterricht haben die Autor(inn)en jüngst vorgelegt (Labuhn, Bögeholz \& Hasselhorn, 2008). Im Rahmen einer quasi-experimentellen Interventionsstudie entwickelten Lehrer(innen) und Wissenschaftler(innen) gemeinsam eine achtstündige Unterrichtseinheit zum Thema Ernährung mit Sachwissens- und Selbstregulationsvermittlung. Die Resultate des Prätest-Posttest-Vergleichs sprechen dafür, dass es bei zusätzlicher Förderung selbstregulierten Lernens im Vergleich zu Unterricht auf ausschließlich Sachwissensbasis zu einer Steigerung der Selbstregulation kommt. Gleichzeitig lernte die Selbstregulationsgruppe trotz reduzierter Unterrichtszeit für fachbezogene Inhalte ebenso viel über Ernährung wie die Vergleichsgruppe. In einer anschließenden neuen und für beide Gruppen identischen Unterrichtseinheit war ein Lernvorteil der Trainingsgruppe festzustellen. Da im Schulkontext der Nutzen derartiger Ansätze besonders hoch einzuschätzen ist, wenn nicht lediglich kurzfristige Effekte erzielt werden, sondern Lernen und Leistungen dauerhaft gesteigert werden können, bestand das zentrale Anliegen der vorliegenden Arbeit darin, die langfristigen Effekte dieser unterrichtsintegrierten Selbstregulationsförderung auf Selbstregulation und fachspezifische Leistung zu prüfen.

\section{Herausforderungen für den Schulunterricht.}

Von einer gelungenen unterrichtsintegrierten Förderung selbstregulierten Lernens sollten alle Teilnehmenden profitieren. Den in der pädagogisch-psychologischen Interventionsforschung häufig zu findenden „Matthäuseffekt“ gilt es zu vermeiden. Unter diesem Effekt versteht man, dass die ohnehin leistungsstarken Schüler(innen) - bzw. Schüler(innen) mit hoher Selbstregulationskompetenz - mehr von den Fördermaßnahmen profitieren als die eher benachteiligten. Wegen des hohen Sprachanteils von Selbstregulationstrainings könnte die Wirksamkeit auch vom Migrationsstatus der Kinder abhängen. Wie im Rahmen von PISA 2000 (Baumert et al., 2001) festgestellt, scheinen sich sprachliche Defizite kumulativ auf Performanzen in Sachfächern auszuwirken. PISA 2006 bringt alarmierende Resultate hervor: Im Bereich der Naturwissenschaften finden sich besonders in Deutschland große Kompetenzunterschiede zwischen Jugendlichen ohne und Jugendlichen mit Migrationshintergrund (Prenzel et al., 2007). 
Unseres Wissens liegen bislang keine Befunde zur differentiellen Wirkung unterrichtsintegrierter Selbstregulationsförderung vor. Ziel dieses Beitrages war es daher auch, für die von Labuhn et al. (2008) wirksam umgesetzte unterrichtsintegrierte Selbstregulationsförderung zu prüfen, ob differentielle Trainingseffekte auftreten. Außerdem sollte die langfristige Wirkung der vorgestellten Intervention untersucht werden. Folgenden Fragen wurde im Einzelnen nachgegangen:

1. Ist die unterrichtsintegrierte Selbstregulationsförderung langfristig wirksam?

2. Welche Wirkung hat Fachunterricht, der selbstreguliertes Lernen (SRL) systematisch integriert, kurz- und langfristig auf die fachbezogenen Lernleistungen?

3. Gibt es selbstregulations- oder fachbezogene Unterschiede in der Wirkung der Intervention für Subgruppen
a) mit bzw. ohne Migrationshintergrund
b) mit unterschiedlichen Ausgangsvoraussetzungen im selbstregulierten Lernen?

\subsubsection{Methode}

Design

Die Beantwortung der aufgeworfenen Fragen nach den langfristigen und differentiellen Wirkungen der unterrichtsintegrierten Selbstregulationsförderung erfolgte im Rahmen eines Prätest-Posttest-Follow-up-Designs mit Kontrollgruppe. Die sieben teilnehmenden Klassen wurden auf zwei experimentelle Bedingungen aufgeteilt. Maßgeblich für die Einteilung war die Bereitschaft der Lehrkräfte, an der Studie mitzuwirken und ihren Unterricht entsprechend zu gestalten. Vier Klassen bildeten die Trainings- und drei die Kontrollgruppe. In allen Klassen fand eine achtstündige curricular valide Biologie-Unterrichtseinheit im Fach Naturwissenschaften zum Thema „Ernährung“ statt. In der Trainingsgruppe (TG) war diese mit kontextspezifischen Anregungen der Selbstregulation verknüpft. Die Kontrollgruppe (KG) wurde ebenfalls acht Stunden zu dem gleichen Thema, lediglich ohne explizite Anregung selbstregulierten Lernens, unterrichtet.

\section{Stichprobe}

Die Unterrichtsforschung erfolgte in Zusammenarbeit mit einer integrierten Gesamtschule in Nordrhein-Westfalen. Zum ersten Messzeitpunkt umfasste die Stichprobe $N=199$ Schüler(innen) (TG: 115; KG: 84), davon 94 Mädchen und 105 Jungen. Für den zweiten Messzeitpunkt liegen vollständige Daten von 185 (TG: 107; KG: 78) und zum Follow-up von 
161 (TG: 97; KG: 64) Schüler(inne)n vor. Zwischen Trainings- und Kontrollgruppe zeigten sich keine signifikanten Unterschiede hinsichtlich der Merkmale Alter (TG: 13.25; KG: 13.24), Geschlecht (TG: 46\% Mädchen; KG: 48\% Mädchen), Anzahl der Schüler(innen) mit Migrationshintergrund (TG: 39\%; KG: 40\%) und sprachlichen Fähigkeiten, gemessen durch die Verbalskala V1 des KFT (Heller \& Perleth, 2000) (TG: 11.94; KG: 11.98).

\section{Biologie-Unterrichtseinheit mit Selbstregulationselementen}

Bei dem naturwissenschaftlichen Unterricht („NW“) in der 7. Jahrgangsstufe der teilnehmenden Gesamtschule handelt es sich um eine die Fächer Biologie, Physik und Chemie integrierende Form. Die einzelnen Unterrichtseinheiten weisen meist einen Schwerpunkt eines dieser Fachgebiete auf. Die Entwicklung der Unterrichtseinheit zum Thema Ernährung (Schwerpunkt Humanbiologie) für das Fach Naturwissenschaft erfolgte in enger Kooperation mit den NW-Lehrkräften der 7. Jahrgangsstufe (zum Inhalt der einzelnen Stunden und zur Verknüpfung von Sachwissensvermittlung und Selbstregulationsanregung vgl. Tabelle 4.6).

$\mathrm{Zu}$ Beginn jeder Unterrichtsstunde griff die Lehrkraft kurz das Thema der vorherigen Stunde auf und erfragte zentrale Inhalte. Am Ende jeder Unterrichtsstunde hatten die Schüler(innen) die Aufgabe, drei bis vier - auf das in der Stunde behandelte Selbstregulationselement bezogene - Merksätze zu formulieren, aufzuschreiben und in einen speziell für die Unterrichtseinheit vorgesehenen Ordner zu heften. Während der gesamten Unterrichtseinheit wurden die Schüler(innen) wiederholt dazu ermutigt, die neu erlernten Selbstregulationselemente auch bei der Bearbeitung von Hausaufgaben sowie in anderen Fächern anzuwenden. 
Tabelle 4.6. Inhalte der Unterrichtseinheit Ernährung und Elemente der integrierten SRL-Förderung

\begin{tabular}{lll}
\hline Stunde & Thema & SRL-Element \\
\hline 1 & Warum essen und trinken wir? & Zielsetzung \\
& Bau- und Betriebsstoffe / Energie & \\
& Zusammensetzung einer Mahlzeit & Zielsetzung \\
& Energiegehalt und Nährstoffanalyse, Nähr- & Self-monitoring \\
& stoffverteilung & Selbstreflexion \\
3 & Bedeutung der Nährstoffe im Körper & Volitionale Strategien: \\
& (Kohlenhydrate, Fette, Eiweiße, Wirkstoffe) & Selbstmotivierung, \\
& & Aufrechterhalten des Arbeitsprozesses \\
5 & Fortsetzung der Stunde 3 & \\
& Energiegehalt / Leben kostet Energie & Volitionale Strategien: \\
6 & Grundumsatz / Leistungsumsatz & Umgang mit Ablenkung \\
& Konsequenzen aus GU/LU & Lernstrategien: \\
7 & Zu dick / zu dünn, BMI & Ressourcen und Lernumgebung \\
& Ausgewogene Ernährung, Ernährungspyra- & Selbstreflexion, \\
& mide, & Ist-Soll-Abgleich \\
& Planung eines ausgewogenen Frühstücks & \\
& Durchführung eines ausgewogenen Früh- & Wiederholung: \\
& stücks & Volitionale Strategien und \\
& & Selbstreflexion \\
\hline
\end{tabular}




\section{Stunde, „Ziele und Zielsetzung“}

Die Unterrichtsstunde begann mit einem informierenden Einstieg und Überblick über die Inhalte der Unterrichtseinheit. Alle Schüler(innen) erhielten dazu eine Übersicht sowie einen Ordner zum Abheften sämtlichen Materials für diese Einheit. Ausgehend von dem Plan für die Unterrichtseinheit wurde das Thema „Ziele und Zielsetzung“ eingeführt und diskutiert, welchen Zweck Ziele erfüllen. Die Schüler(innen) sammelten Beispiele für realistische und unrealistische Ziele und diskutierten, welche Wirkung verschiedene Zielformulierungen haben können. Für die Bearbeitung von Aufgaben zu dem Thema „Warum essen und trinken wir? - Bau- und Betriebsstoffe/ Energie“" setzten sich die Schüler(innen) individuelle Ziele. Gegen Ende der Stunde wurden die Aufgaben im Klassenverband besprochen. Dabei berichteten die Schüler(innen) auch über ihre zuvor gesetzten Ziele und ihre Zielerreichung in Bezug auf die Aufgabeninhalte.

\section{Stunde, ,Self-monitoring und Selbstreflexion“}

Themen der Stunde waren die Zusammensetzung einer Mahlzeit, der Energiegehalt von Lebensmitteln und die Nährstoffanalyse. Vor Bearbeitung der Aufgaben zu diesem Bereich stellte die Lehrkraft der Klasse so genannte Leitfragen zur Beobachtung des eigenen Lernprozesses vor: „1. Was ist mein Ziel für diese Aufgabe? 2. Arbeite ich konzentriert? 3. Habe ich mein Ziel erreicht?" Die Anwendung dieser Leitfragen wurde anhand eines Beispiels besprochen. Dann bearbeiteten die Schüler(innen) ein Arbeitsblatt, wobei sie versuchen sollten, die Fragen selbstständig anzuwenden. Zum Schluss der Stunde wurden die inhaltlichen und die auf das Selbstregulationselement - die Beobachtung des eigenen Lernens - bezogenen Resultate besprochen.

\section{3. und 4. Stunde, ,, Volitionale Strategien“}

Die Stunden wurden als Doppelstunde unterrichtet. Der fachliche Schwerpunkt, die Bedeutung der Nährstoffe im Körper, wurde mit dem Selbstregulationselement verbunden, das auf Selbstmotivierung und das Aufrechterhalten des eigenen Arbeitsprozesses zielte. Im ersten Teil der Stunde wurde ein Unterrichtsgespräch mit der gesamten Klasse geführt. Die Lehrkraft stellte zu Beginn die Kernfragen „Wie kann man sich selbst zum Arbeiten motivieren, auch wenn man keine große Lust hat?“, sowie „Wie kann man „dranbleiben“, auch wenn man lieber andere Dinge tun würde?“. Die Schüler(innen) formulierten schließlich individuelle Selbstinstruktionen (,persönliche Formeln“), die ihnen in Bezug auf diese beiden Fragen 
hilfreich erschienen. Bei der anschließenden Bearbeitung der fachbezogenen Aufgaben setzten sie ihre persönlichen Formeln nach Bedarf ein.

\section{Stunde, , Umgang mit Ablenkung“}

Thema der Stunde waren die Bedeutung und Berechnung des Grund- und Leistungsumsatzes, das damit verknüpfte Selbstregulationselement - als weitere volitionale Strategie war der Umgang mit Ablenkung vom Arbeitsprozess. Zu Beginn fand erneut ein Unterrichtsgespräch statt. Die Diskussion beleuchtete entsprechend des Selbstregulationselements die Frage „Was kann mich von der Arbeit ablenken?“. Die Schüler(innen) sammelten in Kleingruppenarbeit Strategien zur Abschirmung von Ablenkung, die dann bei der Bearbeitung eines Arbeitsblattes zum Einsatz kamen. In der Ergebnisdiskussion wurden die fachbezogenen Inhalte sowie die individuellen Strategien und deren Erfolg vorgestellt. Im Rahmen einer Hausaufgabe wurden die Schüler(innen) dazu motiviert, ihre persönlichen Formeln und Strategien anzuwenden.

\section{Stunde, „Ressourcen und Lernumgebung“}

Zunächst wurden die Hausaufgaben besprochen und kurze Berichte über die Anwendung der individuellen Strategien und persönlichen Formeln gegeben. Das Selbstregulationselement „Ressourcen und Lernumgebung“ wurde im direkten Anschluss an die Hausaufgabenbesprechung eingeführt und hatte mit der Frage des Arbeitsplatzes zu Hause einen direkten Bezug dazu. Die dann thematisierten Inhalte „Konsequenzen aus Grund- und Leistungsumsatz" knüpften an die vorherige Stunde an. Als Hausaufgabe sollten Erkenntnisse über die günstige Gestaltung des Arbeitsplatzes durch erste Schritte umgesetzt werden.

\section{Stunde, „Reflexion“}

$\mathrm{Zu}$ Beginn der siebten Stunde wurde das Thema „Lernumgebung“ wieder aufgegriffen. Die Schüler(innen) berichteten über ihren eigenen Arbeitsplatz, Verbesserungsmöglichkeiten und die konkreten nächsten Schritte. Dann fand eine Reflexion über die bisherige Unterrichtseinheit statt, die von den Fragen „Was haben wir bereits gelernt?““, „Haben wir das schon gut verstanden?“ und „Gibt es noch Wissenslücken, und wie kann man mit diesen umgehen?" geleitet wurde. In Gruppenarbeit formulierten die Schüler(innen) dann konkrete Fragen und schlossen die Wissenslücken durch gemeinsame Diskussion, Nachlesen in verschiedenen bereitgestellten Büchern und direkten Fragen an die Lehrkraft. Zum Schluss überprüften sie, ob alle offenen Fragen geklärt werden konnten. Als Hausaufgabe organisierten die 
Schüler(innen) in Kleingruppen ein ausgewogenes Frühstück. Jede(r) Schüler(in) hatte die Aufgabe, einen Bestandteil der Mahlzeit vorzustellen. Dabei waren das Wissen über Ernährung und die bekannten Selbstregulationselemente (wenn möglich) anzuwenden.

\section{Stunde, „Wiederholung der Selbstregulationselemente“}

Das geplante Frühstück wurde in Verknüpfung mit einer Wiederholung der in der Unterrichtseinheit behandelten Komponenten selbstregulierten Lernens durchgeführt. Die Schüler(innen) saßen in Kleingruppen zusammen und stellten sich gegenseitig die Lebensmittel vor, für die sie sich entschieden hatten. Nach einiger Zeit regte die Lehrkraft ein Gespräch über die in den letzten Stunden behandelten Strategien und „persönlichen Formeln“ an. Die Schüler(innen) tauschten sich darüber aus, ob und wie ihnen diese hilfreich waren und gaben sich gegenseitig Tipps und Verbesserungsvorschläge. Zum Abschluss formulierte die Klasse gemeinsam Merksätze zu den zentralen Selbstregulationselementen. Die Schüler(innen) notierten diese Sätze und gestalteten ein individuelles Merkblatt.

\section{Durchführung}

Die achtstündige Unterrichtseinheit erstreckte sich sowohl für Trainings- als auch Kontrollgruppe über drei Wochen. Vor Beginn der Einheit wurde in einem Prätest der Selbstregulationsfragebogen vorgelegt. Im direkten Anschluss an die Unterrichtseinheit erfolgte der Posttest, der sowohl den Selbstregulationsfragebogen als auch einen fachbezogenen Test zu dem behandelten Thema Ernährung umfasste. In einer Follow-up Erhebung sechs Monate später kamen erneut der Selbstregulationsfragebogen und der fachbezogene Test zum Wissen über Ernährung in unveränderter Form zum Einsatz.

\section{Messinstrumente}

\section{a) Demographische Informationen}

Neben Alter und Geschlecht der teilnehmenden Schüler(innen) wurde außerdem nach der Herkunft beider Elternteile gefragt. In Anlehnung an PISA 2003 (Prenzel et al., 2006) wurden nur die Schüler(innen), deren beide Elternteile in Deutschland geboren wurden, als „ohne Migrationshintergrund“ bezeichnet. Jugendliche mit mindestens einem aus einem anderen Land stammenden Elternteil wurden als „,mit Migrationshintergrund“ klassifiziert. 


\section{b) Sprachliche Fähigkeiten}

Zur Kontrolle der verbalen Fähigkeiten der Schüler(innen) zogen wir die Unterskala „Wortschatz“ (Verbalskala V1) des Kognitiven Fähigkeitstests (KFT; Heller \& Perleth, 2000) heran. Es waren maximal 25 Punkte zu erreichen. Die Erfassung der verbalen Fähigkeiten in der vorliegenden Studie erfolgte lediglich mit dem Ziel, die Vergleichbarkeit von Trainingsund Kontrollgruppe hinsichtlich der sprachlichen Fähigkeiten zu prüfen. Diese Variable ging nicht in die weiteren Analysen mit ein.

\section{c) Selbstregulationsfragebogen}

Der Selbstregulationsfragebogen entsprach mit geringfügigen Anpassungen dem Messinstrument, das Perels et al. (2005) im Rahmen der Evaluation eines Selbstregulationstrainings einsetzten. Alle Items waren in Aussageform formuliert und auf einer vierstufigen Ratingskala ( 1 = stimmt gar nicht, $2=$ stimmt eher nicht, $3=$ stimmt eher, $4=$ stimmt genau $)$ zu beantworten. Der Fragebogen umfasste die Subskalen „Motivation/Volition“ (32 Items, $\alpha=.89$ ), „Lernstrategien“ (25 Items, $\alpha=.87$ ), „Selbstwirksamkeitserwartungen“ (12 Items, $\alpha=76$ ), „Ziele“ (7 Items, $\alpha=.49$ ) und „Selbstregulationsstrategien“ (9 Items, $\alpha=.62$ ). Diese Komponenten selbstregulierten Lernens stehen in engem Bezug zu den Inhalten der Unterrichtsintervention in der TG. Der in der Ausgangsuntersuchung (Labuhn et al., 2008) gefundene mittlere Zusammenhang zwischen den Subskalen deutet darauf hin, dass es sich um durchaus voneinander unterscheidbare Aspekte selbstregulierten Lernens handelt, die jedoch in einer Höhe zusammenhängen, die auch die Aggregierung zu einem Gesamtwert für Selbstregulation $(\alpha=$.94) rechtfertigt. Aufgrund der unzureichenden Reliabilität der Subskala „Ziele“( $\alpha=.49)$ verzichteten wir auf weitere Auswertungen dieses Teilbereichs.

\section{d) Erfassung des Sachwissens}

Der Test zur Erfassung des fachbezogenen Wissens wurde angelehnt an das Unterrichtsthema Ernährung in Form eines Lückentexts konzipiert (vgl. Tabelle 4.7). Dieser erfasste das Verständnis für zentrale Konzepte der Unterrichtseinheit, die durch 14 auszufüllende Lücken erfragt wurden $(\alpha=.78)$.

Tabelle 4.7. Itembeispiel für den lehrzielorientierten Test zum Thema Ernährung

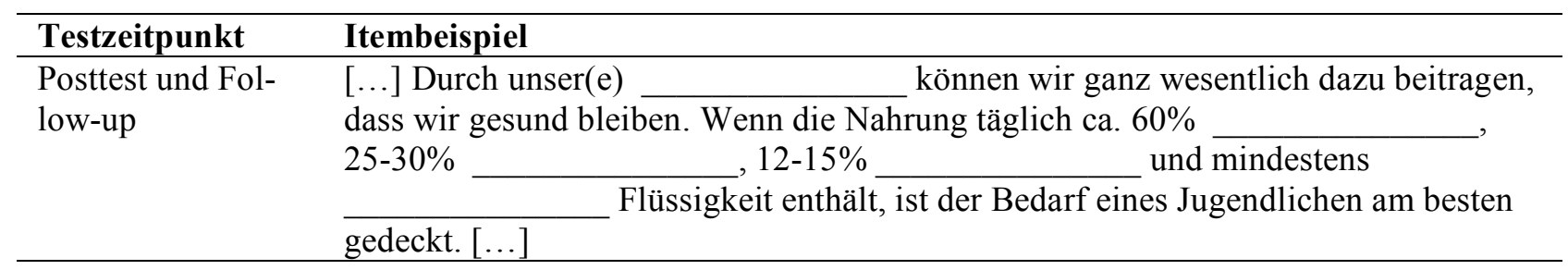




\subsubsection{Ergebnisse}

Um die Resultate der vorliegenden Studie in den größeren Kontext der Programmevaluation einordnen zu können, geben wir zunächst einen kurzen Überblick über die Ergebnisse der Ausgangsuntersuchung (Labuhn et al., 2008).

Im direkten Anschluss an die Intervention wurde eine insgesamt positive Wirkung der in den naturwissenschaftlichen Unterricht integrierten Selbstregulationsförderung festgestellt. Der Gesamtwert Selbstregulation stieg in der Trainingsgruppe signifikant an. Der tendenzielle Effekt auf der Skala Selbstwirksamkeit und der signifikante Effekt auf der Skala Lernstrategien wiesen darauf hin, dass in diesen Bereichen ein Aufbau von Selbstregulation angestoßen worden war. Die fachbezogenen Lernleistungen während der Interventionsphase unterschieden sich in Trainings- und Kontrollgruppe nicht signifikant voneinander. Die hier berichteten Ergebnisse basieren im Wesentlichen auf den zusätzlich sechs Monate nach dem Posttest erhobenen Follow-up-Daten.

\section{Langfristige Wirksamkeit der Selbstregulationsförderung}

Die langfristige Wirksamkeit der unterrichtsintegrierten Förderung selbstregulierten Lernens wurde auf zwei Ebenen geprüft: Zunächst auf der Ebene der direkt auf die Interventionsinhalte bezogenen Selbstregulationskomponenten (Frage 1), dann auf der für den Fachunterricht relevanten Ebene des Erwerbs von Sachwissen (Frage 2).

Tabelle 4.8 sind die Mittelwerte und Standardabweichungen der Selbstregulationsskalen getrennt nach Trainings- und Kontrollgruppe für Prätest, Posttest und Follow-up zu entnehmen. Im Prätest lagen keine signifikanten Gruppenunterschiede auf der Gesamtskala SRL $(F(1,159)=1.892, p=.171)$, sowie auf den Subskalen (Motivation: $F(1,159)=2.523$, $p=.114$; Lernstrategien: $F(1,159)=0.311, p=.578$; Selbstwirksamkeit: $F(1,159)=2.081$, $p=.151$; Selbstregulationsstrategien: $F(1,159)=2.211, p=.139)$ vor.

Zur Überprüfung der ersten Frage wurden für die in der Follow-up-Testung gemessenen abhängigen Variablen des Selbstregulationsfragebogens einfaktorielle Varianzanalysen durchgeführt. Für den Gesamtwert Selbstregulation zeigte sich dabei ein signifikanter Effekt zugunsten der Trainingsgruppe $\left(F(1,159)=4.752, p=.031\right.$, eta $\left.^{2}=.029\right)$. Auf der Ebene der Subskalen resultierten ein signifikanter Effekt für die Skala Motivation $(F(1,159)=5.529$, $p=.020$ eta $\left.^{2}=.034\right)$, sowie ein signifikanter Effekt für die Skala Selbstwirksamkeit $\left(F(1,159)=7.952, p=.005\right.$, eta $\left.^{2}=.048\right)$. In beiden Fällen lag der Mittelwert der Trainingsgruppe über dem der Kontrollgruppe. 
Die oben genannten Unterschiede zwischen Trainings- und Kontrollgruppe in Bezug auf die Skalen zum selbstregulierten Lernen waren im Prätest zwar nicht signifikant, jedoch auch nicht vollständig zu vernachlässigen. Wir überprüften die Effekte daher zusätzlich kovarianzanalytisch. Die Kontrolle der Ausgangswerte änderte nichts am prinzipiellen Ergebnismuster, jedoch waren die Effekte für den Gesamtwert Selbstregulation $(F(1,158)=$ $2.843, p=.094$, eta $\left.^{2}=.018\right)$ sowie die Subskala Motivation $\left(F(1,158)=2.943, p=.088\right.$, eta $^{2}$ $=.018$ ) nur noch auf dem 10\%-Niveau nachweisbar. Der Effekt für die Subskala Selbstwirksamkeit blieb auf dem 5\%-Niveau erhalten $\left(F(1,158)=5.839, p=.017\right.$, $\left.^{2}=.036\right)$.

Tabelle 4.8. Mittelwerte (und Standardabweichungen) der Selbstregulationsskalen für Trainings- und Kontrollgruppe für Prätest, Posttest und Follow-up

\begin{tabular}{|c|c|c|c|c|c|}
\hline \multirow[t]{2}{*}{ Abhängige Variablen } & & \multicolumn{2}{|c|}{ Trainingsgruppe } & \multicolumn{2}{|c|}{ Kontrollgruppe } \\
\hline & & $\mathrm{M}$ & (SD) & $\mathrm{M}$ & (SD) \\
\hline \multirow[t]{3}{*}{ Motivation } & Prätest & 2.84 & $(.42)$ & 2.73 & $(.42)$ \\
\hline & Posttest & 2.93 & $(.43)$ & 2.78 & $(.49)$ \\
\hline & Follow-up & 2.83 & $(.44)$ & 2.67 & $(.41)$ \\
\hline \multirow[t]{3}{*}{ Lernstrategien } & Prätest & 2.86 & $(.42)$ & 2.82 & $(.38)$ \\
\hline & Posttest & 2.96 & $(.44)$ & 2.84 & $(.52)$ \\
\hline & Follow-up & 2.83 & $(.43)$ & 2.74 & $(.46)$ \\
\hline \multirow[t]{3}{*}{ Selbstwirksamkeit } & Prätest & 2.83 & $(.38)$ & 2.74 & $(.44)$ \\
\hline & Posttest & 2.93 & $(.39)$ & 2.76 & $(.46)$ \\
\hline & Follow-up & 2.88 & $(.43)$ & 2.68 & $(.44)$ \\
\hline \multirow[t]{3}{*}{ Selbstregulation } & Prätest & 2.72 & $(.43)$ & 2.62 & $(.46)$ \\
\hline & Posttest & 2.81 & $(.46)$ & 2.64 & $(.57)$ \\
\hline & Follow-up & 2.74 & $(.45)$ & 2.63 & $(.47)$ \\
\hline \multirow[t]{3}{*}{ Gesamtskala SRL } & Prätest & 2.84 & $(.33)$ & 2.77 & $(.33)$ \\
\hline & Posttest & 2.93 & $(.36)$ & 2.79 & $(.42)$ \\
\hline & Follow-up & 2.83 & $(.37)$ & 2.70 & $(.37)$ \\
\hline
\end{tabular}

Die zweite Frage bezog sich auf die langfristige Wirkung der Selbstregulationsanregung auf die fachbezogene Leistung. Sechs Monate nach der Intervention zeigte sich in der einfaktoriellen Varianzanalyse ein signifikanter Effekt zugunsten der Trainingsgruppe ( $F$ (1, $159)=9.137, p=.003$ eta $\left.^{2}=.054\right)$. Tabelle 4.9 zeigt die Mittelwerte und Standardabweichungen für den fachbezogenen Test zum Posttest- und Follow-up-Messzeitpunkt. 
Bei gemeinsamer Betrachtung der Resultate zu unseren ersten beiden Fragen ist festzuhalten, dass für die unterrichtsintegrierte Selbstregulationsförderung sechs Monate nach der Intervention sowohl auf der Ebene der Selbstregulation, als auch auf der Ebene des Sachwissens langfristige Effekte nachgewiesen werden können.

Tabelle 4.9. Mittelwerte (und Standardabweichungen) der fachbezogenen Tests für Trainings- und Kontrollgruppe

\begin{tabular}{|c|c|c|c|c|c|}
\hline \multirow[t]{2}{*}{ Abhängige Variable } & & \multicolumn{2}{|c|}{ Trainingsgruppe } & \multicolumn{2}{|c|}{ Kontrollgruppe } \\
\hline & & $\mathrm{M}$ & (SD) & $\mathrm{M}$ & (SD) \\
\hline $\begin{array}{l}\text { Sachwissen Ernährung } \\
\text { (max. } 14 \text { Punkte) }\end{array}$ & Posttest & 5.70 & 3.17 & 5.70 & 3.30 \\
\hline $\begin{array}{l}\text { Sachwissen Ernährung } \\
\text { (max. } 14 \text { Punkte) }\end{array}$ & Follow-up & 6.96 & 3.79 & 5.05 & 5.13 \\
\hline
\end{tabular}

\section{Differentielle Wirksamkeit}

Mit dem Ziel zu prüfen, ob bestimmte, für den Schulkontext relevante Subgruppen unterschiedlich stark von der Intervention profitieren, untersuchten wir, ob die Wirkung der Intervention auch für Kinder mit Migrationshintergrund bzw. mit unterdurchschnittlichen Ausgangsfähigkeiten in der Selbstregulation („Baseline-SRL“) festzustellen ist (Frage 3). Zur Berechnung der Gruppierungsvariable „Baseline-SRL“ zogen wir die Selbstregulationsfähigkeit vor der Intervention heran und teilten die Gesamtgruppe per Mediansplit in stärkere und schwächere „Selbstregulierer“. Für die Baseline-Selbstregulation zeigten sich keine Unterschiede zwischen Schüler(inne)n mit und ohne Migrationshintergrund $(\mathrm{p}=.400)$.

Die differentiellen Analysen erfolgten in einem ersten Schritt für die kurzfristige Wirkung der Intervention auf den Gesamtwert Selbstregulation sowie die Leistung (Posttest) und in einem zweiten Schritt für die langfristige Wirkung (Follow-up). Für die abhängige Variable Selbstregulation ergab eine 2 (Messzeitpunkt) x 2 (Gruppe) x 2 (Migrationshintergrund) x 2 (Baseline-SRL)-faktorielle Messwiederholungsvarianzanalyse einen Haupteffekt des Messzeitpunktes $\left(F(1,176)=5.124, p=.031\right.$, eta $\left.^{2}=.026\right)$ und erwartungsgemäß einen Haupteffekt der Baseline-Selbstregulation $\left(F(1,176)=229.696, p=.000\right.$, eta $\left.^{2}=.566\right)$. Über beide experimentellen Gruppen hinweg zeigten sich höhere Werte für die bereits anfänglich „starken Selbstregulierer“. Die Analyse ergab außerdem eine signifikante Interaktion der Faktoren Messzeitpunkt und Gruppe $\left(F(1,176)=4.708, p=.031\right.$ eta $\left.^{2}=.026\right)$. Post-hoc-Analysen 
belegten einen signifikanten Anstieg für die Trainingsgruppe $(t(108)=-3.521, p=0.001, r=$ $0.321)$, nicht aber für die Kontrollgruppe $(t(77)=-0.380, p=0.705, r=0.043)$. Alle anderen Interaktionen und Haupteffekte waren nicht statistisch bedeutsam. Es waren somit weder eine unterschiedliche Trainingswirkung in Abhängigkeit von Migrationshintergrund noch von der Baseline-Selbstregulation nachzuweisen.

Die Wirksamkeit im Hinblick auf Unterschiede in der fachbezogenen Posttestleistung wurde mittels 2 (Gruppe) x 2 (Migrationshintergrund) x 2 (Baseline-SRL)-faktorieller Varianzanalyse überprüft. Diese ergab lediglich einen Haupteffekt des Migrationshintergrundes ( $F$ $(1,176)=15.719, p=.000$, eta $\left.^{2}=.082\right)($ vgl. Abb. 4.3a). Schüler(innen) ohne Migrationshintergrund schnitten besser ab als Schüler(innen) mit Migrationshintergrund. Kein weiterer Effekt erreichte bei dieser Analyse das Signifikanzniveau.

Für die im Follow-up gemessene Selbstregulation ergab die 2 (Gruppe) x 2 (Migrationshintergrund) x 2 (Baseline-SRL)-faktorielle Varianzanalyse einen Haupteffekt der Baseline-Selbstregulation $\left(F(1,150)=78.988, p=.000\right.$, eta $\left.^{2}=.35\right)$. Der Mittelwert der Schüler(innen) mit höheren Ausgangswerten lag erwartungsgemäß über dem der Schüler(innen) mit niedrigen Ausgangswerten.

Die differentielle Analyse der fachbezogenen Leistung im Follow-up erfolgte mittels 2 (Gruppe) x 2 (Migrationshintergrund) x 2 (Baseline-SRL)-faktorieller Varianzanalyse. Es resultierte, wie gemäß der oben dargestellten Ergebnisse zu erwarten, ein signifikanter Haupteffekt der Gruppe $\left(F(1,150)=4.951, p=.028\right.$, eta $\left.^{2}=.032\right)$. Trainierte Schüler(innen) schnitten besser $\mathrm{ab}$ (s.o.). Es zeigte sich außerdem eine signifikante Interaktion von Gruppe und Migrationshintergrund $\left(F(1,150)=4.687, p=.032\right.$, eta $\left.^{2}=.030\right)$. Der Vorteil der Trainingsgruppe gegenüber der Kontrollgruppe war nur bei Schüler(inne)n ohne Migrationshintergrund nachzuweisen $\left(F(1,99)=15.503, p=.000\right.$, eta $\left.^{2}=.135\right)$, nicht aber bei Kindern mit Migrationshintergrund. Bei Betrachtung der Mittelwerte (vgl. Abb. 4.3b) wird jedoch deutlich, dass der signifikante Vorsprung der Trainingsgruppe auf ein unerwartetes Absinken der Leistung der Schüler(innen) ohne Migrationshintergrund - sogar noch unter den Wert derer mit Migrationshintergrund in der Kontrollgruppe zurückgeht.

Insgesamt entsprechen die Ergebnisse zur dritten Frage unserer Erwartung, dass auch potenziell benachteiligte Schüler(innen) von der Selbstregulationsförderung im Unterricht profitieren. Erwartungsgemäß lagen die Mittelwerte der zu Beginn „starken Selbstregulierer“ in Posttest und Follow-up über denen der zu Beginn ,schwachen Selbstregulierer“. Bezüglich des Zugewinns an Selbstregulation durch die Intervention zeigten sich jedoch keine Unterschiede in Abhängigkeit vom Ausgangsniveau der Selbstregulation oder dem Migrationshin- 
tergrund. Im Hinblick auf die fachbezogene Leistung muss die Feststellung der vergleichbaren Ergebnismuster für die untersuchten Subgruppen jedoch eingeschränkt werden: Hier stellten wir einen Vorteil der Schüler(innen) ohne Migrationshintergrund im Posttest fest.
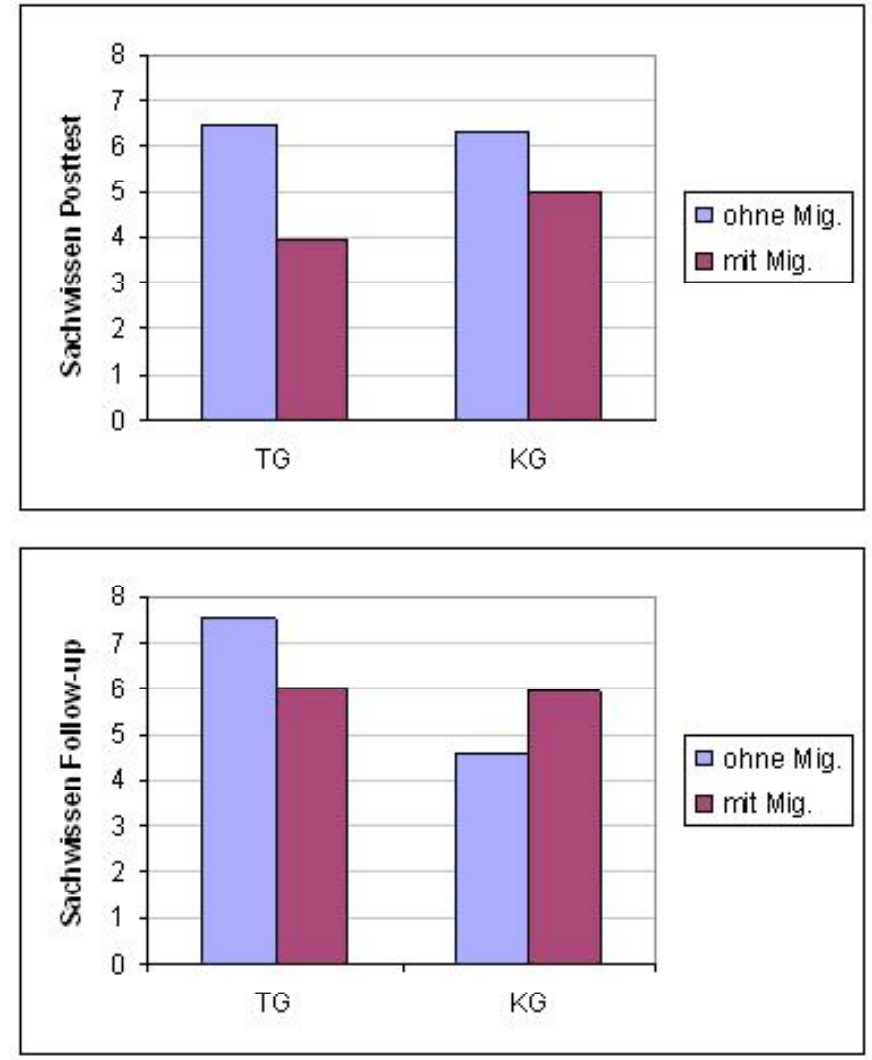

Abbildung 4.3. a) Haupteffekt Migrationshintergrund im fachbezogenen Posttest und b) Interaktion Gruppe x Migrationshintergrund im fachbezogenen Follow-up Test

\subsubsection{Diskussion}

Die Untersuchung der langfristigen Wirksamkeit der Selbstregulationsförderung im naturwissenschaftlichen Unterricht bezog sich auf die sechs Monate nach der Intervention festzustellende Wirkung auf die Selbstregulation der Schüler(innen) (Frage 1) und deren fachbezogene Lernleistung (Frage 2). Darüber hinaus wurde überprüft, ob Schüler(innen) mit und ohne Migrationshintergrund sowie mit unterschiedlichen Ausgangsvoraussetzungen bezüglich der Selbstregulation vergleichbar von der Intervention profitieren (Frage 3).

Im direkten Anschluss an die Intervention hatten Labuhn et al. (2008) einen statistisch bedeutsamen Vorteil der Trainingsgruppe für den Gesamtwert Selbstregulation berichtet. Wie die hier präsentierten Follow-up-Analysen ergaben, ließ die durch das Training erzielte Wir- 
kung in ihrer Höhe über einen Zeitraum von sechs Monaten wieder nach. Das ist durchaus erwartungsgemäß, da die erworbenen selbstregulativen Fertigkeiten möglicherweise im Unterricht zunehmend weniger abgerufen werden, wenn nicht entsprechend förderliche Bedingungen geschaffen und der Transfer auf andere Themen unterstützt wird. Trotz der Abnahme blieb der Vorteil der Trainings- gegenüber der Kontrollgruppe über einen Zeitraum von sechs Monaten jedoch bestehen. Dieser Befund steht im Einklang mit der Interventionsstudie von Perels et al. (2005), die ebenfalls von einem stabilen Gruppenunterschied berichten. Zum Teil geht der Unterschied in der vorliegenden Studie allerdings auf ein stärkeres Absinken der Werte in der Kontrollgruppe zurück.

Der im direkten Anschluss an die Förderung gefundene Vorteil der Trainingsgruppe für die Subskala Lernstrategien zeigte sich im Follow-up nicht mehr. Möglicherweise ist dies auf mangelnde oder gänzlich fehlende Transfer- und Trainingsmöglichkeiten für strategisches Lernen im regulären Schulalltag zurückzuführen. Strategien bedürfen einer regelmäßigen und konstanten Einübung, damit sie routiniert ausgeführt werden und ihre Wirkung auf der Leistungsebene entfalten können. Dafür müssten im Unterricht ausreichend Übungsgelegenheiten geschaffen werden. Dass es sich lohnen würde, diese verstärkt bereitzustellen, zeigt der deutliche kurzfristige Zugewinn in diesem Bereich bei den trainierten Schüler(inne)n.

Während für die Selbstwirksamkeit unmittelbar nach der Intervention lediglich ein tendenzieller Vorteil der Trainingsgruppe berichtet worden war, war dieser in der vorliegenden Überprüfung nach sechs Monaten statistisch bedeutsam. Ein ähnliches Muster zeigte sich für die Motivation. Ein direkter Interventionseffekt war nicht nachzuweisen, im Follow-up jedoch zeigte sich ein Vorteil der Trainingsgruppe. Das Resultat deutet darauf hin, dass durch die Intervention im motivationalen Bereich eine Entwicklung angestoßen wurde, die sich über einen längeren Zeitraum und über die Trainingsphase hinaus fortsetzt. Wenngleich handlungsnahe Fertigkeiten, wie etwa Lernstrategien, aufgrund fehlender Übungsgelegenheiten in ihrem Auftreten abnehmen, so ist anzunehmen, dass motivationale Variablen einem anderen Wirkmechanismus unterliegen. Möglicherweise war die erzielte Wirkung im direkten Anschluss an die Interventionsphase noch nicht festzustellen, weil sich Veränderungen im Motivsystem von Kindern selten innerhalb kurzer Zeit vollziehen (vgl. Hasselhorn \& Gold, 2006, S. 97). Es ist anzunehmen, dass durch die unterrichtsintegrierte Förderung den Schüler(inne)n Strategien zur Reflexion des eigenen Lernens und Arbeitens mit auf den Weg gegeben wurden, deren Wirkung sich im Sinne eines „Schläfereffekts“ erst in der wiederholten Auseinandersetzung mit persönlich bedeutsamen leistungsbezogenen Situationen im Alltag entfaltet. Im Gegensatz zu der Anwendung von Lernstrategien ist die Weiterentwicklung hier mögli- 
cherweise weniger an konkrete Unterrichtssituationen gebunden und obliegt stärker dem individuell wahrgenommenen Nutzen der in der Intervention vermittelten Elemente (z.B. Selbstmotivierung). Diese Interpretation legt nahe, dass der Frage möglicherweise unterschiedlicher Wirkungen der Förderung (lern)strategischer und motivationaler Komponenten selbstregulierten Lernens zukünftig verstärkt nachgegangen werden sollte.

Die zweite in diesem Beitrag fokussierte Frage bezog sich auf die langfristige Wirkung der Selbstregulationsförderung hinsichtlich des in der Unterrichtseinheit erworbenen fachbezogenen Wissens. Im direkten Anschluss an die Intervention lagen keine leistungsbezogenen Unterschiede zwischen Trainings- und Kontrollgruppe vor (Labuhn et al., 2008). Bei der wiederholten Leistungsmessung sechs Monate nach der Intervention schnitt die Trainingsgruppe besser ab. Darüber hinaus lag der Mittelwert des fachbezogenen Tests über dem im direkten Anschluss an die Unterrichtseinheit gemessenen Wert. Die Behaltensleistung der Trainingsgruppe überstieg damit die Wiedergabe des Gelernten kurz nach der Unterrichtseinheit. Grund dafür könnte ein erlangtes tieferes Verständnis der vermittelten Konzepte sein, welches sich dann - möglicherweise auch unterstützt durch die in der Zwischenzeit dazugelernten weiteren Fakten - in einer zusätzlich verbesserten Leistung widerspiegelt. Dieses Muster zeigte sich in der Kontrollgruppe nicht. Auf Unterschiede in den zwischenzeitlich im Unterricht behandelten Themen ist dies wahrscheinlich nicht zurückzuführen: Die an der Studie beteiligten Lehrkräfte unterrichteten auch nach der Intervention weiterhin in den Gruppen und teilten uns mit, dass entsprechend des gemeinsamen Curriculums in allen Klassen die gleichen Themen behandelt wurden und sie sich untereinander über die Inhalte regelmäßig austauschten. Natürlich bleibt ohne eine tatsächliche Überprüfung der Inhalte der Grad der Übereinstimmung des Unterrichtsgeschehens über die sechs Monate hinweg offen. Aufgrund der uns vorliegenden Informationen ist aber von einer hinreichenden Vergleichbarkeit auszugehen. Wir sehen in dem Befund daher einen Hinweis darauf, dass die unterrichtsintegrierte Selbstregulationsförderung langfristig $\mathrm{zu}$ einer positiven Lernentwicklung im Bereich Fachwissen beigetragen hat.

Schließlich wurde auch differentiellen Trainingswirkungen für im Schulkontext relevante Subgruppen nachgegangen. Eine dieser in der vorliegenden Studie betrachteten Subgruppen bildeten die Schüler(innen) aus Familien mit Migrationshintergrund. Ob ein Kind aus einer solchen Familie stammt, hatte für die direkte Wirkung der Selbstregulationsförderung keine Bedeutung. Auch Schüler(innen) aus Elternhäusern, in denen häufig Deutsch nicht die erste gesprochene Sprache ist, wurden in ihrer Selbstregulation gefördert. Dies werten wir als einen Erfolg der Maßnahme, da es aufgrund des hohen Sprachanteils des Trainings zur Be- 
nachteiligung dieser Kinder hätte kommen können. Fachbezogen schlägt sich dieser Rückstand auch tatsächlich nieder: Im Posttest lagen die Leistungen der Schüler(innen) mit Migrationshintergrund hinter denen aus Familien, in denen beide Elternteile aus Deutschland stammen. Dies traf unabhängig davon zu, ob die Schüler(innen) das Training durchliefen oder nicht. In der Follow-up Testung zeigte sich ein differentieller Effekt, der sich darin niederschlug, dass der deutliche Vorteil der trainierten Schüler(innen) im fachbezogenen Test nicht für Kinder mit Migrationshintergrund nachzuweisen war. Dieser Interaktionseffekt war jedoch in erster Linie auf ein unerwartetes Absinken der Leistung der Schüler(innen) ohne Migrationshintergrund in der Kontrollgruppe zurückzuführen, so dass ein signifikanter Unterschied zur Trainingsgruppe resultierte. Als eine Benachteiligung der Kinder mit Migrationshintergrund kann dieser Befund demnach nicht interpretiert werden. Auffällig bleibt jedoch, dass für die Schüler(innen) aus Migrantenfamilien kein erkennbarer Leistungsunterschied zwischen Trainings- und Kontrollgruppe zu erkennen war. Offensichtlich hatte der auf die Selbstregulation bezogene Kompetenzzuwachs durch das Training bei Kindern mit Migrationshintergrund noch nicht die Wirksamkeitsschwelle für fachliches schulisches Lernen überstiegen. In zukünftigen Studien im Bereich unterrichtsintegrierter Förderung von Lernkompetenzen sollte ein verstärktes Augenmerk auf die speziellen Bedürfnisse solcher Subgruppen, z.B. auf deren potenzielle sprachliche Defizite, gelegt werden.

Die Überprüfung differentieller Interaktionseffekte bezog sich weiterhin auf die Untersuchung der Interventionswirkung bei Schüler(inne)n mit starken bzw. schwachen Ausgangsvoraussetzungen im selbstregulierten Lernen. In der vorliegenden Studie zeigte sich kein Matthäuseffekt. Auch Schüler(innen), die vor der Intervention zu den „schwachen Selbstregulierern“ zählten, profitierten trotz der ungünstigeren Ausgangslage gleichermaßen von der Förderung. Ebenso langfristig und bezogen auf die Entwicklung der fachbezogenen Leistung waren keine Unterschiede festzustellen, die auf das ursprüngliche Selbstregulationsniveau zurückgingen. Wir betrachten dies als ein für die unterrichtsintegrierte Förderung positives Resultat, da es ganz besonders im Schulkontext und insbesondere auch nach TIMSS und PISA von hoher Relevanz ist, Schüler(innen) mit unterschiedlichsten Ausgangsvoraussetzungen und sprachlichen Fähigkeiten gleichermaßen $\mathrm{zu}$ fördern und Chancengleichheit $\mathrm{zu}$ gewährleisten. Eine unterrichtsintegrierte Förderung kann nur unter diesen Bedingungen als tatsächlich erfolgreich bezeichnet werden.

Die vorliegende Studie unterliegt natürlich einigen Beschränkungen. Eine solche ist in der recht geringen Intensität der Intervention zu sehen. Durchschnittlich standen maximal 15 Minuten in jeder der nur acht Unterrichtsstunden für die Vermittlung von Selbstregulations- 
elementen zur Verfügung, was sich vermutlich in den lediglich kleinen Effektstärken widerspiegelt. Zusätzlich stand die Berücksichtigung schulischer Alltagsbedingungen einer randomisierten Gruppenzuweisung entgegen. Die Tatsache, dass sich keine Gruppenunterschiede hinsichtlich des Ausgangsniveaus in den untersuchten abhängigen Variablen zeigten und das gewählte Vorgehen eine ökologisch valide Einbettung der Intervention in den Schulalltag erlaubte, ließ es uns akzeptabel erscheinen, diese methodische Schwäche in Kauf zu nehmen.

Im Rahmen unserer Realisierung der unterrichtsintegrierten Förderung wurde keine explizite Überprüfung des von den Lehrkräften tatsächlich umgesetzten Unterrichts im Sinne der Treatment-Integrität vorgenommen. Mit dem Ziel, möglichst realistische Unterrichtsbedingungen beizubehalten, beschränkten wir uns auf Checklisten, auf denen die Lehrkräfte nach jeder Stunde Fragen zum Ablauf des Unterrichts und der Umsetzung der geplanten Inhalte beantworteten. Es wurde vorher deutlich gemacht, dass diese Checklisten nicht der Beurteilung der Leistung der Lehrkräfte, sondern lediglich unserem wissenschaftlichen Interesse an der Realisierbarkeit der Vorgaben dienten und möglichst ehrlich ausgefüllt werden sollten. Die Auswertung ergab weder in den Trainings- noch in den Kontrollklassen erkennbare Abweichungen von den geplanten Stundenverläufen. In zukünftigen Studien wäre zu überlegen, welcher Gewinn durch den Einsatz von Videoaufnahmen oder geschulten Beobachter(inne)n zu erzielen wäre.

Ein weiterer Kritikpunkt bezieht sich auf die Erhebungsmethode. Zwar setzten wir mit der Leistungsmessung ein objektives Kriterium ein, die Feststellung der Selbstregulation erfolgte jedoch aus ökonomischen Gründen durch einen Fragebogen im Selbstaussageformat. Ein solches wirft Zweifel an der Validität der Messung auf. Bei kritischer Betrachtung wird auf diese Weise nicht das tatsächliche Regulationsverhalten gemessen, sondern lediglich die diesbezügliche Einschätzung der Schüler(innen) (vgl. Artelt, 1999, 2000). Der retrospektive Selbstbericht kann durch ein möglicherweise durch die Intervention erhöhtes Bewusstsein für das Thema Strategien (im Gegensatz zu deren tatsächlichen Nutzung) und/oder durch die Tendenz, im Sinne sozialer Erwünschtheit zu antworten, verzerrt werden. Bei der Interpretation der Ergebnisse der Fragebogenmessung ist dementsprechend gewisse Vorsicht geboten. Für zukünftige unterrichtsbezogene Selbstregulationsforschung wäre es wünschenswert, die Messung stärker kontextspezifisch zu gestalten und fertigkeitsbezogene Maße zur Erfassung strategischen Lernens wie z.B. Strategietests und Arbeitsproben einzusetzen, die genauere Rückschlüsse auf die tatsächliche Selbstregulationskompetenz erlauben. Es wäre weiterhin denkbar, die Messung um zusätzliche Perspektiven zu erweitern, etwa um die Einschätzung der Selbstregulation der Schüler(innen) durch deren Lehrkräfte, z.B. auch in anderen Fächern. 
Ohnehin wäre es im Schulalltag von hohem Wert, den Transfer der Selbstregulation auf andere Fächer und Inhalte zu unterstützen. Mit dem Ziel, transferförderliche Bedingungen zu schaffen, wären Schulungsmaßnahmen für und eine intensivere Vernetzung der Fachlehrkräfte vorstellbar. Darüber hinaus zeigten sich Lerntagebücher bereits als sehr geeignet für die „online-Messung“ der Entwicklung selbstregulatorischer Fertigkeiten sowie als die Intervention unterstützendes Element (z.B. Perels, Gürtler \& Schmitz, 2005; Schmitz \& Wiese, 2006). Eine weitere Herausforderung wäre auch die längsschnittliche Begleitung von Klassen, in deren Schulalltag die Förderung integriert wird, möglichst in Kombination mit prozessualer Evaluation (vgl. Schmitz, 1990; Schmitz \& Skinner, 1993). Die Ergebnisse der vorliegenden Studie stimmen hinsichtlich des Nutzens der Integration selbstregulierten Lernens in den naturwissenschaftlichen Unterricht optimistisch und regen zu weiterführenden Fragestellungen an. Vertiefende Forschung besonders unter Einbezug von Kindern mit Migrationshintergrund wäre insbesondere nach den neusten PISA-Ergebnissen wünschenswert. 


\subsection{Kritisches Resümee zu Studie 1}

Für die Integration der Selbstregulationsförderung in den naturwissenschaftlichen Unterricht, deren Evaluation in den empirischen Beiträgen der Kapitel 4.1 und 4.3 dargestellt wurde, ist zusammenfassend festzuhalten, dass

1.) selbstreguliertes Lernen innerhalb des regulären Unterrichts kurzfristig verbessert werden kann und die Effekte zum Teil zeitlich stabil sind,

2.) fachbezogenes Lernen während der Intervention nicht von der reduzierten Unterrichtszeit beeinträchtigt und langfristig durch die Selbstregulationsförderung sogar positiv beeinflusst wird,

3.) Schüler(innen) mit unterschiedlichen Lernvoraussetzungen sowie Schüler(innen) mit und ohne Migrationshintergrund gleichermaßen von der Förderung profitieren.

Trotz der insgesamt positiven und im Hinblick auf die Integration der Selbstregulationsförderung in den Schulalltag ermutigenden Resultate sollen auch die praktischen (Kapitel 4.4.1) und methodischen Grenzen (Kapitel 4.4.2) des realisierten Ansatzes kritisch betrachtet werden. Dabei sollen einige in den beiden empirischen Beiträgen bereits angesprochene Aspekte fokussierter thematisiert und weitere diskussionswürdige Punkte erörtert werden.

\subsubsection{Praktische Grenzen der Unterrichtsintervention}

\section{Dauer und Intensität der Förderung}

Die vorgestellte Unterrichtseinheit bestand aus insgesamt 8 Schulstunden (à 45 Minuten), in denen jeweils etwa 15 Minuten für die Selbstregulationsförderung zur Verfügung standen. Im regulären Unterricht darf selbstverständlich die Vermittlung fachbezogener Inhalte nicht zu kurz kommen, damit bleibt jedoch für die explizite Einbindung von Trainingselementen wenig Zeit. Durch die gewählte kontextspezifische und an schulische Alltagsbedingungen angepasste Form fand die Intervention auf einem recht geringen Intensitätslevel statt. Dies spiegelt sich u.a. in den geringen Effektstärken wider. Gleichzeitig ist ein solcher Befund ermutigend, da er darauf hindeutet, dass bei intensiverer Förderung die Wirkung deutlich gesteigert werden könnte. Die Selbstregulationsförderung belief sich lediglich auf eine Unterrichtseinheit von acht Schulstunden und endete mit Abschluss des Projektes. Nach der Intervention kam es über das Schulhalbjahr hinweg zu einem deutlichen Absinken der gemessenen Selbstregulation. Aus sozial-kognitiver Perspektive ist ein solcher Befund erwartungsgemäß: 
In der Trainingsphase konnte zwar der Aufbau von Selbstregulationsfähigkeiten angestoßen werden, damit diese aber auch langfristig ins Verhaltensrepertoire übernommen werden, bedarf es vielfacher Anwendungs- und Übungsmöglichkeiten. Diese standen jedoch nicht in ausreichendem Maße, und vor allem nicht ausdrücklich im Unterricht angeboten, zur Verfügung. Damit fehlten die notwendigen begünstigenden Kontextfaktoren für eine Aufrechterhaltung der Selbstregulationsfähigkeiten auf dem durch die Intervention erreichten Level. Die explizite Anregung zur weiteren Anwendung des erworbenen Verhaltens war im Rahmen der Studie nicht vorgesehen, die empirischen Befunde (das Absinken der Mittelwerte) belegen aber zusätzlich die Bedeutung dieser Implikation der sozial-kognitiven Theorie für Fördermaßnahmen im Schulkontext.

Vom Standpunkt der Theorie aus könnte man zudem auch die Lehrenden als Lernende betrachten, die die Elemente der Selbstregulationsförderung erst über eine längere Zeit und wiederholte Anwendungsgelegenheiten hinweg in ihr Verhaltensrepertoire übernehmen. Routiniertes Instruktionsverhalten könnte die Intensität der unterrichtsintegrierten Förderung und damit auch die erzielten Effekte erhöhen.

\section{Inhaltsspektrum der Intervention}

Die dargestellte Unterrichtseinheit umfasste eine relativ große Bandbreite an integrierten Selbstregulationskomponenten. Ziel dieser Konzeption war es, möglichst viele Teilaspekte des zyklischen Selbstregulationsprozesses (Zimmerman, 2000) mit Trainingselementen abzudecken (vgl. Perels et al., 2005). Eine solche breit angelegte Auswahl an Trainingsinhalten kann jedoch auch zuungunsten einer fokussierten Förderung einzelner Strategien gehen. Bei einer Interventionsdauer von 15 Minuten pro Unterrichtsstunde in einer achtstündigen Einheit wird deutlich, dass nicht viel Zeit für eine intensive Förderung der einzelnen Selbstregulationselemente zur Verfügung stand. Kritisch könnte berechtigterweise angemerkt werden, dass die Trainingsbestandteile in der kurzen zur Verfügung stehenden Zeit lediglich eingeführt, nicht aber geübt und automatisiert werden konnten. Die Breite des Inhaltsspektrums wirkt sich damit einschränkend auf die Intensität der Intervention aus. Wie oben bereits erläutert, wird dadurch das Erreichen eines hohen Niveaus der Selbstregulationsfähigkeit gemäß dem Entwicklungskonzept nach Zimmerman (2000) unwahrscheinlich. Möglicherweise führte die Einführung der Vielzahl von Komponenten (Zielsetzung, Motivation, Lernstrategien, Selbstreflexion, volitionale Strategien) dazu, dass zuviel neue Information innerhalb kurzer Zeit präsentiert und damit das Verhalten nicht langfristig aufgebaut wurde: Zwar blieb der im Anschluss an die Intervention festgestellte Unterschied im Gesamtwert für selbstreguliertes 
Lernen zwischen Trainings- und Kontrollgruppe über den Zeitraum von sechs Monaten stabil, der gemessene Wert sank jedoch deutlich ab. Besonders auffällig zeigt sich dieses Resultat in dem verhaltensbezogenen Indikator „Lernstrategien“. Die Trainingsphase hatte kurzfristig zu einem signifikanten Anstieg in diesem Bereich geführt, der langfristig aber nicht mehr festzustellen war, was auf fehlende Anwendungsmöglichkeiten zurückzuführen sein könnte.

Motivationale Variablen wie die Selbstwirksamkeitserwartungen scheinen dagegen einem anderen Wirkmechanismus $\mathrm{zu}$ unterliegen und weniger stark von konkreten Anwendungsmöglichkeiten abhängig zu sein (was plausibel ist, da verhaltensbezogene Variablen per definitionem stärker mit konkreter Anwendung, also der Verhaltensausführung verbunden sind). Durch die unterrichtsintegrierte Förderung motivationaler Aspekte haben die Schüler(innen) vermutlich Möglichkeiten der Betrachtung des eigenen Lernprozesses aufgebaut, deren Wirkung sich in der wiederholten Auseinandersetzung mit persönlich bedeutsamen leistungsbezogenen Situationen im Alltag entfaltete. Diese sind im regulären Unterricht natürlicherweise gegeben und müssen nicht explizit geschaffen werden. Für die motivationalen Trainingskomponenten scheint das breite Inhaltsspektrum also ein geringeres Problem darzustellen. Hier ist offenbar bereits der Anstoß durch das Training wirksamer und setzt eine individuelle Weiterentwicklung in Gang. Grundsätzlich bleibt die Frage, ob eine fokussierte Förderung eines begrenzten Inhaltsbereiches (wie z.B. Lern- und Selbstregulationsstrategien bei den Elzen-Rump \& Leutner, 2007 sowie Leopold, den Elzen-Rump \& Leutner, 2006) oder die Förderung einer größeren Bandbreite an Selbstregulationskomponenten (Labuhn et al., 2008; Perels et al., 2005) wirksamer ist, so lange offen, wie sie nicht einer konkreten empirischen Überprüfung unterzogen wird. Es ist aber zu vermuten, dass besonders die verhaltensbezogenen Komponenten einer intensiveren Förderung und häufigerer Anwendungsgelegenheiten bedürfen, die eine breit angelegte Förderung in einem kurzen Zeitraum nicht bereitstellen kann. Für die theoriegeleitete Selbstregulationsforschung, der ein Interesse am genauen Verständnis der Prozesse selbstregulierten Lernens zugrunde liegt, bietet sich der breit angelegte Ansatz weniger gut an. Mit dem Ziel, Subprozesse und ihre Zusammenhänge genau zu analysieren, erscheint es sinnvoll, einen Teilbereich einer fokussierten Untersuchung zu unterziehen. 


\section{Transfer der Selbstregulationsfähigkeiten}

Die Frage des möglichen Transfers der erworbenen Selbstregulationselemente wurde in der vorgestellten Studie lediglich als eine Teilfrage behandelt (vgl. Kapitel 4.1), ist aber aus grundlagenwissenschaftlicher Perspektive die zentrale Frage. Das entsprechende Resultat, dass trainierte Schüler(innen) in einer der Trainingsphase folgenden, in beiden Gruppen identisch präsentierten Unterrichtseinheit einen Lernvorteil zeigen, kann vorsichtig als ein Hinweis auf Transferprozesse betrachtet werden. Die Tatsache, dass dieser Lernvorteil festzustellen ist, obwohl in der zweiten Unterrichtseinheit ein Transfer der Selbstregulationsfähigkeiten nicht explizit angeregt wurde, spricht für das Potenzial des Trainingsansatzes. Grundsätzlich erfordert jedoch die Förderung von für schulisches Lernen relevantem Lerntransfer zusätzliche Überlegungen. Eine zentrale Voraussetzung für den Transfer erworbener Fähigkeiten ist das Vorhandensein von Gelegenheiten, die eine Ausführung entsprechenden Verhaltens unter geringem Aufwand möglich machen. In der vorliegenden Studie war es jedoch nicht vorgesehen, die Trainingsinhalte über die Interventionsphase hinaus im Unterricht zu thematisieren und die Schüler(innen) zu deren Anwendung anzuregen. Hier zeigt sich ein Dilemma zwischen aus pädagogisch-psychologischer Sicht wünschenswerten Bedingungen und der Anwendung eines strengen experimentellen Forschungsdesigns: Die Weiterführung der Selbstregulationsförderung in Eigenregie der Lehrkräfte im weiteren Verlauf des Schulhalbjahres war nicht Bestandteil des Designs und wäre unter nicht kontrollierten Bedingungen erfolgt. Im Falle der Evaluation der vorgestellten Unterrichtseinheit hätte ein Rückgriff auf Elemente der Interventionsphase im individuellen Ermessen der Lehrkräfte zu einer Verzerrung der Ergebnisse sowohl von Posttest II (nach der Unterrichtseinheit (UE) „Bewertung“) als auch der Follow-up-Erhebung führen können. Nach Abschluss der beiden Unterrichtseinheiten (vgl. Kapitel 4.1; UE „Ernährung“ und UE „Bewertung“) fand in allen Klassen wieder regulärer Unterricht statt, der nicht mehr in das Forschungsdesign eingebunden war. Dies ermöglichte die in der vorgestellten Studie realisierte weitgehend kontrollierte Evaluation im Hinblick auf die zugrunde liegenden Forschungsfragen - bei gleichzeitig suboptimalen Bedingungen im Hinblick auf den für die Unterrichtspraxis ja eigentlich wünschenswerten Transfer. Eine unter praktischen Gesichtspunkten sinnvolle und nachhaltige Förderung selbstregulierten Lernens hätte zusätzlich transferförderliche Bedingungen verlangt. In Übereinstimmung mit Zimmermans (2000) Konzeption von Entwicklungsniveaus der Selbstregulation müssten auch nach Abschluss des Trainings weiterhin Übungsmöglichkeiten für die trainierten Selbstregu- 
lationsfähigkeiten angeboten und die Schüler(innen) zu deren Aufrechterhaltung und Weiterentwicklung motiviert werden.

Der Transfer selbstregulierten Lernens auf andere Kontexte, Inhalte und Aufgaben hängt nach Pressley et al. (1990) besonders von drei Faktoren ab: a) von dem Wissen über Strategien und Anwendungsmöglichkeiten der Selbstregulation, b) von der Überzeugung, dass Selbstregulation den eigenen Lernprozess positiv beeinflusst und c) von der Fähigkeit, die Selbstregulationsfähigkeiten der Situation entsprechend anzupassen. Der erste Faktor kann durch ein Training (wie z.B. das vorgestellte) abgedeckt, der zweite Faktor kann durch eine kurze Intervention nur zum Teil berücksichtigt werden. Zur Überzeugung, dass die Anwendung von Selbstregulationsfähigkeiten einen persönlichen Nutzen hat und das eigene Lernergebnis verbessert, gelangen Lernende wahrscheinlich erst über einen längeren Zeitraum und die mehrfache Auseinandersetzung mit den Möglichkeiten des erworbenen Verhaltens. Gleiches gilt für den dritten Faktor, der ein Verhalten beschreibt, das dem vierten und höchsten Niveau des Entwicklungskonzepts nach Zimmerman (2000) entspricht: Erst durch Übung und Automatisierung der erlernten Strategien können diese auf andere Kontexte generalisiert und flexibel eingesetzt werden.

Diese Überlegungen beschreiben ein Dilemma zwischen einerseits methodisch korrekten Forschungsdesigns mit möglichst strenger Kontrolle von Störvariablen zur Überprüfung theoretisch und praktisch relevanter Fragestellungen und andererseits Bedingungen im Schulkontext, die - ebenfalls aus theoretischer sowie praktischer Sicht - wünschenswert wären. Sie unterstreichen den Forschungsbedarf zur Vereinbarkeit kontextspezifischer Selbstregulationsförderung mit curricular validem und innovativem Fachunterricht.

\section{Implementation Selbstregulationsförderung in den Unterricht}

An verschiedenen Stellen wurde auf die Notwendigkeit der kontextspezifischen Förderung hingewiesen. Der realisierte unterrichtsintegrierte Ansatz stellt ein gelungenes Beispiel der Verknüpfung fach- und selbstregulationsbezogener Inhalte dar. Die Implementation sorgfältig geplanter, strukturierter Unterrichtsprogramme in den Schulalltag ist jedoch auch mit praxisbezogenen Schwierigkeiten verbunden, die sich in erster Linie auf die Rahmenbedingungen des Schulalltags und die Kooperation von Wissenschaftler(inne)n und Lehrkräften beziehen. Die Durchführung der vorgestellten Unterrichtseinheit erfolgte unter weitgehend ökologisch validen Bedingungen. Die Zuweisung zu Trainings- und Kontrollgruppe erfolgte auf Klassenebene, so dass die Zusammensetzung innerhalb der Lerngruppen bestehen blieb. Die Unterrichtseinheit wurde von den Fachlehrkräften der jeweiligen Klassen zu den gewohn- 
ten, im Stundenplan vorgesehenen Zeiten unterrichtet. Was von wissenschaftlicher Seite nach einem recht geringen Eingriff in den Schulalltag und vielversprechender kontextspezifischer Förderung aussieht, stellte sich jedoch für die teilnehmenden Lehrkräfte, in deren Hand die manualgetreue Umsetzung lag, zum Teil anders dar: Die Notwendigkeit eines starken experimentellen Designs, der plangemäßen Durchführung und genauen Beachtung des Zeitrahmens sowie der Ausschaltung möglicher Störfaktoren - kurz: der stark eingeschränkten Flexibilität - waren ihnen zunächst eher fremd und entsprachen nicht der ihnen vertrauten Unterrichtsrealität. Solche Ansprüche, die aus wissenschaftlicher Perspektive an die Lehrkräfte herangetragen werden, könnten in Zukunft im Vorfeld noch ausführlicher gemeinsam erarbeitet und diskutiert werden.

Die enge Zusammenarbeit von Expert(inn)en aus Wissenschaft und Praxis, die auch Souvignier und Antoniou (2007) als wichtigen Faktor für den Erfolg innovativer Unterrichtsansätze anführen, wäre im Rahmen eines Forschungsprojektes wie dem in der vorliegenden Arbeit vorgestellten sicher noch ausbaufähig. Souvignier und Trenk-Hinterberger (2007) führen zwei zentrale Punkte an, die im Hinblick auf eine Implementation, die in enger Kooperation von Wissenschaftler(inne)n und Lehrkräften (nach der so genannten „symbiotischen Implementationsstrategie“, Gräsel \& Parchmann, 2004) erfolgt, von besonderer Bedeutung sind: Erstens benötigen Lehrkräfte intensive Unterstützung und konkrete Beispiele für die Umsetzung der Konzepte in spezifischen Unterrichtssituationen, und zweitens ist es von großem motivationalen Vorteil, wenn sie selbst erlebt haben, dass solche Unterrichtsansätze wirksam und praktikabel sind. Positive Erfahrungen mit neuen Unterrichtskonzepten stellen eine Voraussetzung dafür dar, dass Lehrkräfte ihre Einstellungen handlungsrelevant verändern (Souvignier \& Trenk-Hinterberger, 2007). Wenngleich in der vorgestellten Untersuchung das hauptsächliche Interesse der wissenschaftlichen Überprüfung der Wirksamkeit des Ansatzes galt und nicht eine langfristige Implementation das erklärte Ziel darstellte, hätten vor allem die beteiligten Lehrkräfte durch eine intensivere Prozessbegleitung zusätzlich profitieren können. Ein stärkerer Fokus auf die Vermittlung der Relevanz der Selbstregulationsförderung und der zugrunde liegenden Konzepte hätte die Wahrscheinlichkeit erhöht, dass sie die Inhalte der Intervention auch langfristig in ihr Verhaltensrepertoire übernommen hätten. In der realisierten Form des Projektes bleiben Wissenschaft und Praxis jedoch über die konkrete Durchführung der Studie hinaus weitgehend isoliert: Von wissenschaftlicher Seite betrachtet wurden die Forschungsfragen beantwortet, von schulpraktischer Seite die Unterrichtseinheit durchgeführt. Weitreichende Veränderungen des Unterrichtsalltags, wie man sie 
von einer Implementationsforschung ${ }^{6}$ erwarten würde, wurden somit nicht bewirkt. Auch wenn dies nicht zu den expliziten Fragen und Zielen der Studie gehörte, so wäre es wünschenswert, in zukünftigen Projekten die Vorteile und Chancen einer engen Kooperation stärker auszubauen und auch mittel- und langfristig nutzbar zu machen; sowohl in Bezug auf die Beantwortung praxisrelevanter Fragen als auch hinsichtlich der Implementation (evaluierten) innovativen Unterrichts.

Die Kritikpunkte, die bereits im Abschnitt zum Transfer angeklungen sind, und die hier um die Perspektive der Implementationsforschung erweitert wurden, gehen streng genommen über die Zielsetzung der kontrollierten Wirksamkeitsüberprüfung des theoretisch begründeten Interventionsansatzes in Studie 1 hinaus. Da das langfristige Ziel solcher Ansätze in der Regel jedoch die Implementation in den Schulkontext darstellt, sollen die hier diskutierten Aspekte Herausforderungen und Chancen auch im Hinblick auf zukünftige Studien aufzeigen.

\subsubsection{Methodische Grenzen der Wirksamkeitsevaluation}

\section{Quasi-experimentelles Design und Gruppenbildung}

In der vorgestellten Untersuchung war es aus praktischen Gründen und unter Berücksichtigung schulischer Alltagsbedingungen nicht möglich eine randomisierte Gruppenzuweisung vorzunehmen. Die Gruppenbildung innerhalb des quasi-experimentellen Designs erfolgte auf Klassenebene, die einzelnen Schüler(innen) wurden somit nicht per Zufall den experimentellen Gruppen zugewiesen, sondern die Klassen blieben als „natürliche Einheiten“ (Rost, 2007, S. 126) bestehen. Diese methodische Schwäche wurde in Kauf genommen, da belegt werden konnte, dass sich die Gruppen nicht hinsichtlich ihres Ausgangsniveaus in den untersuchten abhängigen Variablen unterschieden. Ein solches Vorgehen ermöglichte es zudem, die Intervention ohne jegliche Veränderung der Zusammensetzung der Klassen unter maximal ökologisch validen Bedingungen in den Schulalltag einzubetten. Trotz der plausiblen Gründe, ein solches Forschungsdesign zu wählen, sollen die damit verbundenen Schwierigkeiten nicht unerwähnt bleiben. Der quasi-experimentelle Versuchsplan gewährleistet nicht, dass die

\footnotetext{
${ }^{6}$ Beispiele für Projekte, die einen Implementationsansatz verfolgen, stellen die BMBF-Verbundprojekte Chemie im Kontext (CHiK) und Biologie im Kontext (bik) dar, in denen über mindestens drei Jahre hinweg von Wissenschaftler(inne)n und Lehrkräften gemeinsam innovative Unterrichtskonzepte entwickelt, implementiert und evaluiert werden.
} 
Gruppen vergleichbar sind. Zwar konnte im Rahmen der Studie (siehe Kapitel 4.1 und 4.3) gezeigt werden, dass keine Ausgangsunterschiede in den abhängigen Variablen bestanden, trotzdem ist zu bedenken, dass sich Schulklassen in einer Vielzahl denkbarer Variablen unterscheiden (z.B. Klassenklima, Interaktion mit den Lehrkräften, Gruppenprozesse), welche die Wirkung der Intervention und den Wissenserwerb in einer Unterrichtseinheit beeinflussen können. Da es oftmals nicht möglich ist im Schulkontext maximal kontrollierte experimentelle Bedingungen herzustellen und zugunsten der Realisierbarkeit von Studien auf schwächere Designs (Rost, 2007, S. 117ff.) zurückgegriffen wird, sind solche Einflüsse bei der Interpretation der Befunde quasi-experimenteller Studien stets zu berücksichtigen.

\section{Treatmentintegrität}

Im Rahmen der vorgestellten unterrichtsintegrierten Selbstregulationsförderung wurde keine explizite Überprüfung des von den Lehrkräften tatsächlich umgesetzten Unterrichts im Sinne der Treatment-Integrität vorgenommen. Mit dem Ziel, möglichst realistische Unterrichtsbedingungen beizubehalten, kamen lediglich Checklisten zum Einsatz, auf denen die Lehrkräfte nach jeder Stunde Fragen zum Ablauf des Unterrichts und der Umsetzung der geplanten Inhalte beantworteten. Aufgrund der geringen Anzahl teilnehmender Lehrkräfte waren die Checklisten nicht statistisch auszuwerten. Sie stellten jedoch wichtige Informationen über die Stundenverläufe bereit. Es waren keine nennenswerten Abweichungen von den Inhalten der Manuale zu erkennen. Selbstverständlich stellt ein solches Vorgehen, welches auf Selbstaussagen der Lehrkräfte beruht, weder die Treatmentintegrität sicher noch ersetzt es den Einsatz von Methoden, die Informationen über die manualgetreue Unterrichtsdurchführung liefern können. Dennoch kann der Inhalt der Checklisten als ein Anhaltspunkt für die plangemäße Umsetzung betrachtet werden.

Grundsätzlich ist bei der Durchführung unterrichtsintegrierter quasi-experimenteller Studien zu überlegen, welcher Gewinn durch den Einsatz von Videoaufnahmen oder geschulten Beobachter(inne)n zu erzielen wäre. Solche Methoden der Kontrolle können im ungünstigen Fall die natürliche Unterrichtssituation und damit die ökologische Validität gefährden. Fraglos liefern sie jedoch zusätzliche relevante Informationen, insbesondere über die Vergleichbarkeit der Umsetzung des Unterrichts durch die Lehrkräfte.

Ein weiterer Faktor, der die interne Validität des Versuchsplans gefährden könnte, ist die Kommunikation der Fachlehrkräfte untereinander: Es ist nicht ausgeschlossen, dass die Lehrkräfte der Trainingsklassen Inhalte der Selbstregulationsintervention an die Lehrkräfte, die in den Kontrollklassen regulären Unterricht durchführten, weitergegeben haben. Dieser 
Einwand kann aber in zweierlei Hinsicht entkräftet werden. Zum einen aus praktischer Sicht: Die Lehrkräfte, die in den Kontrollklassen unterrichteten, entschieden sich in der Planungsphase explizit dagegen die zusätzliche Selbstregulationsförderung in den Unterricht einzubinden. Zum anderen aus statistischer Sicht: Sollten in den Kontrollklassen tatsächlich Elemente des Trainings integriert worden sein und hätten diese sogar zu einem Effekt geführt, hätte sich dies in einer reduzierten Differenz zwischen Trainings- und Kontrollgruppe niedergeschlagen. Der Hypothesentest wäre also sehr konservativ (i.S. von ,gegen die Hypothese“) gewesen und hätte mit geringerer Wahrscheinlichkeit Vorteile der Trainingsgruppe aufgedeckt.

\section{Fachbezogene Tests}

Die Ermittlung des in den beiden Unterrichtseinheiten (UE Ernährung und UE Bewertung) erworbenen fachbezogenen Wissens erfolgte durch einen Lückentext (UE Ernährung) und einen Multiple Choice Test (UE Bewertung; siehe Tabelle 4.3). Besonders der Einsatz des Multiple Choice Verfahrens zur Erfassung des in der Unterrichtseinheit „Bewertung“ erworbenen Verständnisses für Inhalte und Zusammenhänge scheint angesichts der Komplexität des Themas suboptimal und gibt Anlass zu Zweifeln an der Validität des nur sieben Items umfassenden Tests. Eine adäquatere Erhebungsmethode für Inhalte dieses Unterrichts stellt ein Verfahren mit offenem Antwortformat dar und wird bei (Eggert \& Bögeholz, submitted) vorgestellt. Aus Gründen der Praktikabilität wurde auf diese ausführlichere, zeitintensivere Methode im Rahmen der vorgestellten Studie verzichtet.

Der im Posttest und im Follow-up eingesetzte Lückentext zur Erfassung des Sachwissens aus der Unterrichtseinheit Ernährung erscheint dagegen eine besser geeignete Messung des erworbenen Wissens darzustellen. Der Test zeigt sich zudem mit einer Reliabilität von $\alpha=.78$ als hinreichend zuverlässig. Dennoch wäre auch in diesem Bereich die Ergänzung der Leistungsmessung durch den Einsatz eines Verfahrens mit offenem Antwortformat wünschenswert, um das tiefe Verständnis für die vermittelten Konzepte detailliert zu überprüfen.

\section{Messung selbstregulierten Lernens}

Einen Schwachpunkt der Studie stellt der alleinige Einsatz eines Fragebogens mit Selbstaussageformat zur Messung der Selbstregulationsfähigkeit der Schüler(innen) dar. Grund für die Auswahl dieser Form der Messung war die ökonomische Einsetzbarkeit (vgl. Spörer \& Brunstein, 2006), die besonders für die Durchführung von Studien im Schulkontext von großer Bedeutung ist. Der Einwand, dass Fragebogenverfahren das individuelle Lernverhalten und dessen Resultate nicht valide abbilden und prognostizieren, wurde an verschiede- 
nen Stellen begründet hervorgebracht (Artelt, 1999, 2000; Baumert, 1993; Kitsantas \& Zimmerman, 2002; Spörer \& Brunstein, 2006; Zimmerman \& Martinez-Pons, 1986). Für den eng mit Selbstregulation verknüpften Bereich der Lernstrategien stellt die Forschungsliteratur kaum eindeutige Antworten auf die Frage bereit, ob und wie sich Lernstrategien im tatsächlichen Lernergebnis widerspiegeln (Artelt, 2000). Nach Baumert und Köller (1996) ist die Korrelation mit Schulleistungsindikatoren in den meisten Studien schwach (siehe auch Baumert, 1993). Ein wichtiger Kritikpunkt an der Erhebung von Lernstrategien und Selbstregulation durch Fragebögen besteht darin, dass die Items häufig keinen oder nur geringen Bezug zu den tatsächlich bearbeiteten Lernaufgaben aufweisen und sehr global formuliert sind („Wenn ich lerne, dann...") (Spörer \& Brunstein, 2006). Leopold und Leutner (2002) zeigen, dass die Spezifität der Itemformulierung die Validität von Fragebögen erhöhen kann. Ein weiteres zentrales Problem, das z.B. Artelt und Schellhas (1996) aufzeigen, ist die Tatsache, dass nicht unbedingt von einem Zusammenhang zwischen dem selbst berichteten Einsatz von Strategien und deren tatsächlicher Anwendung ausgegangen werden kann. Aus einer Studie zum Verhältnis zwischen Strategienennung (Fragebogen) und Strategieanwendung (handlungsnahe Erhebung) geht hervor, dass die prädiktive Validiät von retrospektiven Selbstberichten über das eigene lernstrategische Vorgehen mittels Fragebogen bezweifelt werden kann (Artelt, 1999, 2000). Weder auf Skalen- noch auf Itemebene ließen sich Zusammenhänge zwischen retrospektiver Nennung und tatsächlicher Ausführung von Strategien finden. Ein Großteil der untersuchten Schüler(innen) zeigte eine deutliche Überschätzung der strategischen Performanz, die sich darin äußerte, dass die konkreten Lernverhaltensweisen, denen sie im Fragebogen (teils sogar stark) zustimmten, im Rahmen der handlungsnahen Erfassung nicht festgestellt wurden (Artelt, 2000). Durch ein Selbstaussageverfahren kann also nicht die tatsächliche Selbstregulation, sondern lediglich die diesbezügliche Einschätzung der Schüler(innen) erfasst werden.

Um die Items eines Lernstrategiefragebogens valide beantworten zu können, muss eine Person über das eigene Lernverhalten reflektieren. Ein gewisses Maß an Strategiewissen ist für die korrekte Beantwortung also notwendig (Artelt, 2000). Beim Einsatz von Selbstaussageverfahren in der Lernstrategie- und Selbstregulationsforschung besteht für die Validität der Messung die Gefahr, dass Schüler(innen) zwar über das nötige Strategiewissen verfügen und durchaus auch von der Nutzung spezifischer Strategien berichten, sie jedoch nicht in der Lage sind, die Strategien in entsprechenden Situationen auch adäquat einzusetzen (vgl. Hasselhorn, 1992, 1996). Berichten die Schüler(innen) der Trainingsgruppe nach der Intervention von einem stärkeren Strategieeinsatz und erhöhter Selbstregulation, so kann das auf ein durch 
die Intervention erhöhtes Bewusstsein für diese Trainingsbestandteile zurückgehen, nicht jedoch zwangsläufig auf deren häufigere Anwendung. Beim retrospektiven Berichten und der tatsächlichen Anwendung einer Strategie handelt es sich um zwei qualitativ unterschiedliche Anforderungen (Artelt, 1999, 2000). Das in der Intervention erworbene Wissen kann also durchaus das Antwortverhalten im Fragebogen beeinflussen, welches dem tatsächlichen Regulationsverhalten jedoch nicht entspricht.

Darüber hinaus geht aus der Formulierung des Großteils der Items hervor, welches Verhalten als wünschenswert betrachtet werden könnte (z.B. „Wenn ich mir vorgenommen habe zu lernen, beginne ich damit so schnell wie möglich.“; „Ich versuche mich dazu zu bringen, Hausaufgaben zu erledigen, auch wenn ich keine Lust habe."). Schüler(innen) sind es gewohnt, im Unterrichtskontext möglichst die ,richtigen“ Antworten zu geben. Im retrospektiven Selbstbericht kann damit auch die Tendenz, die Items in sozial erwünschter Richtung zu beantworten die Validität des Verfahrens gefährden. Artelt (2000) vermutet außerdem, dass der retrospektive Selbstbericht über das eigene Lernverhalten zu einem großen Teil allgemeine Einstellungen zum Lernen - und nicht die Anwendungshäufigkeit von Strategien - widerspiegelt. Vor diesem Hintergrund sind die durch die Fragebogenmessung erhaltenen Ergebnisse kritisch zu betrachten. Basierend auf der Annahme, dass theoretisch begründet ein $\mathrm{Zu}-$ sammenhang zwischen Leistungs- und Selbstregulationsmaßen besteht, sollte in zukünftigen Studien der Ansatz von Artelt $(1999,2000)$ aufgegriffen, die Messung stärker kontextspezifisch konzipiert und durch handlungsnahe Analysen ergänzt werden, um vorhandene Beziehungen statistisch abgesichert aufzudecken. Aufschlussreich wäre etwa der Einsatz von Methoden, die Merkmale selbstregulierten Lernens aktualgenetisch, z.B. durch lautes Denken (in der englischsprachigen Literatur ist der Begriff ,think-aloud“ geläufig) (z.B. Bannert, 2005; Veenman \& Beishuizen, 2004), erfassen. Ebenfalls denkbar wäre die Einbeziehung fertigkeitsbezogener Maße zur Erfassung strategischen Lernens wie z.B. Strategietests und Arbeitsproben, die genauere Rückschlüsse auf die tatsächliche Selbstregulationsfähigkeit erlauben. Für dieses multimethodale Vorgehen spricht nach Spörer und Brunstein (2006) auch, dass Fehlerquellen, die jedem einzelnen Verfahren anhängen, durch die Kombination der Methoden aufgedeckt und ggf. ausgeglichen werden können (vgl. Artelt, 2000).

\section{Prozessorientierung und Kontextspezifität}

An die dargestellten methodischen Probleme, die sich aus der Messung selbstregulierten Lernens durch einen Fragebogen im Selbstaussageformat ergeben, schließt sich ein weiterer Kritikpunkt an, der in der theoretischen Konzeption der Selbstregulation begründet liegt. 
Wie in Kapitel 2 ausführlich dargelegt, handelt es sich bei der sozial-kognitiven Vorstellung von selbstreguliertem Lernen um einen zyklischen Prozess aus drei Phasen (Vorbereitungsphase, Handlungsphase, Selbstregulationsphase) (Zimmerman, 2000). Bei der Erfassung selbstregulierten Lernens durch einen Fragebogen werden zwar häufig verschiedene Komponenten des Prozesses durch Subskalen erfasst (z.B. Labuhn et al., 2008; Perels et al., 2005; 2007; in Druck), diese stehen jedoch eher unverbunden nebeneinander und spiegeln das Prozesshafte nicht wider (sondern werden erst im Nachhinein miteinander korreliert). Ein weiteres Problem der in der vorgestellten Studie eingesetzten Messung liegt in der mangelnden Kontextspezifität der Items der Subskalen (Ziele, Motivation, Lernstrategien, Selbstwirksamkeitserwartungen, Selbstregulationsstrategien). Die sozial-kognitive Theorie mit der Betonung sich wechselseitig beeinflussender Prozesse und insbesondere die theorieimmanente Betrachtung selbstregulierten Lernens verlangen streng genommen nach Formen der Messung, die a) kontextspezifisch angelegt sind und b) die angenommenen Prozesse auch abbilden und damit der Forschung zugänglich machen. Beispiele für einen prozessorientierten Forschungsansatz bieten z.B. Schmitz und Wiese (2006) sowie Perels et al. (2007), die durch den Einsatz von Lerntagebüchern über eine Vielzahl von Lernsequenzen hinweg einen Zugang zum Prozess der Selbstregulation finden. Im folgenden Kapitel wird die Frage der kontextspezifischen und prozessorientierten Selbstregulationsforschung aufgegriffen und detaillierter ausgeführt. 


\section{Mechanismen der Wirksamkeit instruktionalen Handelns zur Förderung selbstregulierten Lernens}

\section{1 Überlegungen zur Selbstregulationsforschung auf Prozessebene}

Die prozessorientierte Konzeption selbstregulierten Lernens innerhalb der sozialkognitiven Theorie bedeutet für die Forschung in diesem Bereich, dass konsequenterweise auch die eingesetzten Messinstrumente den zugrunde liegenden Prozess oder zumindest Teile dessen erfassen sollten. Als Gründe für den eher seltenen Einsatz prozessorientierter Erhebungsmethoden nennt Schmitz (2006) u.a. die Natur der Fragestellungen, die oftmals keine Prozessmessung nahelegt, z.B. wenn nach Zusammenhängen zwischen Variablen oder dem Unterschied zwischen Gruppen zu einem definierten Zeitpunkt gefragt wird. Wäre der Fokus der Forschungsfragen ein anderer, etwa der Prozess des Erwerbs einer Fähigkeit oder Fertigkeit, wäre eine an diesem Prozess orientierte Messung die adäquatere Methode. Die Tatsache, dass es sich bei selbstreguliertem Lernen bereits per definitionem um einen Prozess handelt (z.B. Zimmerman, 2000), unterstreicht jedoch den Bedarf an entsprechenden Messmethoden. Als weiteren Grund für den bislang spärlichen Einsatz nennt Schmitz (2006) die Orientierung am Trait-Konzept, die in einem Großteil der pädagogisch-psychologischen Forschung zu finden sei. Die Grundannahme dieses Konzepts, dass Persönlichkeitseigenschaften (traits) zeitlich relativ stabil sind und sich über die Zeit lediglich geringfügig ändern, spiegelt sich in den eingesetzten Fragebögen wider, die solche Eigenschaften oder Verhaltensdispositionen erfassen. Die sozial-kognitive Theorie und Forschung ist dagegen vom Interesse an situationsbezogenen (Lern-)Prozessen in Individuen geleitet, also sehr viel stärker an den sogenannten States orientiert. Vertreter(innen) der Theorie plädieren daher für die mikroanalytische Messung der selbstregulativen Prozesse, die während des Lernens oder der Aufgabenausführung ablaufen (z.B. Bandura, 1997). Diese Methode wird mit dem Ziel eingesetzt, einen möglichst direkten Zugang zu Gedanken und Überzeugungen unmittelbar während oder in engem zeitlichem Zusammenhang mit der Ausführung bestimmter Verhaltensweisen (z.B. der Bearbeitung einer Mathematikaufgabe) zu erhalten. In der Regel werden Personen einzeln untersucht und durch an die jeweilige Aufgabe angepasste Messinstrumente kontextspezifische Informationen erhoben. Die Messung zentraler Prozessvariablen wie Selbstwirksamkeitserwartungen, Selbsteinschätzung oder Selbstbewertung erfolgt damit an Schlüsselstellen des Lern- oder Bearbeitungsprozesses. Der mikroanalytische Ansatz wurde bereits erfolgreich herangezogen um den Selbstregulationsprozess fokussiert zu analysieren und theoretische Annahmen zu 
validieren (Cleary \& Zimmerman, 2001; Kitsantas, Zimmerman \& Cleary, 2000; Kitsantas \& Zimmerman, 2002, 2006; Zimmerman \& Kitsantas, 1997, 1999). Kitsantas und Zimmerman (2002) heben als großen Vorteil dieser Herangehensweise hervor, dass kontextspezifische Fragen für die Befragten weitaus weniger missverständlich sind und darüber hinaus größere prädiktive Validität besitzen als die, besonders im Rahmen von Gruppenerhebungsverfahren eingesetzten, global formulierten Items in Selbstaussageverfahren (vgl. Tab. 5.1). Diesen liegt häufig die Annahme stabiler Persönlichkeitsmerkmale (traits) zugrunde, welchen aber aus sozial-kognitiver Sicht nur eine geringe erklärende Funktion und wenig Vorhersagekraft für situationsspezifisches Verhalten zukommt.

Tabelle 5.1. Gegenüberstellung der globalen und kontextspezifischen Erfassung von Selbstregulationsvariablen

\begin{tabular}{|c|c|c|}
\hline Variable & $\begin{array}{l}\text { Erfassung durch globales Fragebogen- } \\
\text { item }^{\text {a }}\end{array}$ & $\begin{array}{l}\text { kontextspezifische Erfassung } \\
\text { (Bezug auf konkrete Aufgabe) }\end{array}$ \\
\hline $\begin{array}{l}\text { Selbstwirksamkeits- } \\
\text { erwartung }\end{array}$ & $\begin{array}{l}\text { Auch schwierige Aufgaben stellen für } \\
\text { mich keine unüberwindbaren Hindernis- } \\
\text { se dar. } \\
\text { (Ratingskala) }\end{array}$ & $\begin{array}{l}\text { Wie sicher bist du dir, dass du diese } \\
\text { Aufgabe richtig lösen kannst? } \\
\text { (Ratingskala) }\end{array}$ \\
\hline Zielsetzung & $\begin{array}{l}\text { Wenn ich mir einmal ein Ziel gesetzt } \\
\text { habe, lasse ich mich nicht leicht davon } \\
\text { abbringen. } \\
\text { (Ratingskala) }\end{array}$ & $\begin{array}{l}\text { Was ist Dein Ziel für die Bearbeitung } \\
\text { dieser Aufgabe? } \\
\text { (offenes Antwortformat) }\end{array}$ \\
\hline Interesse & $\begin{array}{l}\text { Wenn ich in [Schulfach] etwas Neues } \\
\text { dazulernen kann, bin ich bereit, auch } \\
\text { Freizeit dafür zu verwenden. (Ratingska- } \\
\text { la) }\end{array}$ & $\begin{array}{l}\text { Wie interessant findest du diese Aufgabe } \\
\text { im Vergleich zu anderen Mathe- } \\
\text { aufgaben, die Du schon gerechnet hast? } \\
\text { (Ratingskala) }\end{array}$ \\
\hline Selbstreflexion & $\begin{array}{l}\text { Ich denke häufig über mein Lernverhal- } \\
\text { ten nach. } \\
\text { (Ratingskala) }\end{array}$ & $\begin{array}{l}\text { Hast du, während du die Aufgabe gelöst } \\
\text { hast, darüber nachgedacht, ob du auf } \\
\text { dem richtigen Weg bist? } \\
\text { (Ratingskala) }\end{array}$ \\
\hline $\begin{array}{l}\text { Umgang mit Fehlern, } \\
\text { Adaption }\end{array}$ & $\begin{array}{l}\text { Fehler zeigen mir, was ich anders ma- } \\
\text { chen könnte. } \\
\text { (Ratingskala) }\end{array}$ & $\begin{array}{l}\text { Wenn du solche Aufgaben nochmal } \\
\text { rechnen musst, würdest du beim nächs- } \\
\text { ten Mal etwas anders machen? } \\
\text { (offenes Antwortformat) }\end{array}$ \\
\hline
\end{tabular}

Anmerkungen.

${ }^{\mathrm{a}}$ z.B. bei Labuhn et al. (2008) und Perels et al. (2005)

${ }^{\mathrm{b}}$ z.B. bei Labuhn, Zimmerman \& Hasselhorn (submitted)

An dieser Stelle ist zu erwähnen, dass der Einsatz von Fragebögen mit eher situationsübergreifenden Iteminhalten nicht gleichzusetzen ist mit einer zugrunde liegenden TraitOrientierung oder grundsätzlich fehlender Kontextspezifität, sondern insbesondere im Schul- 
kontext eine ökonomische Methode darstellt, die viele Studien (z.B. die in dieser Arbeit vorvorgestellte, Labuhn et al., 2008 oder Perels et al., 2007) überhaupt erst ermöglicht. Die Methode der Mikroanalyse erlaubt es jedoch durch Einzeluntersuchungen und die Prozessorientierung, das Lernen und die Ausführung von Aufgaben sehr viel fokussierter zu untersuchen. Im Rahmen mikroanalytisch ausgerichteter Studien (z.B. Kitsantas \& Zimmerman, 2002, 2006; Chen \& Zimmerman, 2007) wurden große Effektstärken und außergewöhnlich hohe Korrelationen zwischen psychologischen Variablen und Leistungsmaßen berichtet. Diese Zusammenhänge bleiben in Fragebogenuntersuchungen häufig hinter den Erwartungen zurück und lassen Zweifel an der Validität der Messinstrumente aufkommen (vgl. Kapitel 4.2.2 zur Validitätsproblematik von Selbstaussageverfahren). Auch in der im Rahmen der vorliegenden Arbeit dargestellten Studie 1 (Kapitel 4) zeigten sich nur geringe Korrelationen zwischen Leistungs- und Selbstregulationsmaßen (die höchste signifikante Korrelation betrug $r=.20$ ). Aufgrund der theoretisch plausiblen Annahme existierender Zusammenhänge zwischen den entsprechenden Variablen sollte die Erhebungsmethode in solchen Studien angesichts der deutlichen Vorteile kontextspezifischer Prozeduren überdacht werden.

Mikroanalytische Erfassungsmethoden haben nicht nur für die Erforschung von Lernprozessen aus wissenschaftlicher Sicht einen hohen Wert: Sie liefern auch im Schulkontext praxisrelevante Informationen, die herkömmliche Verfahren zur Leistungsmessung und Tests zur Erfassung fachbezogenen Wissens nicht bereitstellen. Cleary und Zimmerman (2006) stellen mit dem Strategy Microanalytic Assessment (SMA) eine alternative, in der sozialkognitiven Theorie verankerte Erhebungsmethode vor, mit der motivationale, metakognitive und behaviorale (d.h. in diesem Falle lernstrategische) Prozesse kontextspezifisch erfasst werden. Dieser ökologisch valide Ansatz soll Überzeugungen und Herangehensweisen von Schüler(inne)n während der Ausführung spezifischer Aufgaben in bestimmten Kontexten bzw. Schulfächern ermitteln. Verschiedene Messinstrumente zur Erhebung aufgabenbezogener motivationaler Überzeugungen (z.B. Selbstwirksamkeitserwartungen), selbstregulativer Prozesse (z.B. Selbsteinschätzung und Selbstbewertung) sowie der Anwendung spezifischer Strategien (z.B. bestimmte Bearbeitungsschritte, Behaltensstrategien) kommen zum Einsatz. Die Methode liefert damit detaillierte Informationen über Lern- und Verstehensprozesse, in die herkömmliche Tests zur Leistungsmessung keine Einblicke geben (z.B. Lernverhalten, Motivation, Selbsteinschätzung), und die es Lehrer(inne)n ermöglichen Schüler(inne)n eine mehrere Ebenen umfassende Förderung (d.h. kognitiv, motivational und behavioral) anzubieten. Cleary und Zimmerman (2006) berichten, dass Lehrkräfte die Methode des SMA im Vergleich zu herkömmlichen Formen der Erfassung von Leistungsdaten im Schulkontext im Hin- 
blick auf die Planung von Interventionen und Ansätzen der individuellen Förderung als informativer und nützlicher beurteilen, sie aber gleichzeitig sehr viel weniger vertraut mit deren Anwendung sind. Auf schulbezogener sowie auf wissenschaftlicher Ebene scheint es also den deutlichen Bedarf zu geben, prozessorientierte, kontextspezifische Messmethoden zu entwickeln, einzusetzen und zu diskutieren.

\subsection{Prozessorientierte Forschung im Unterrichtskontext: Zur Bedeutung von Bewer- tungsstandards und Feedback im Selbstregulationsprozess}

Wie in den Kapiteln 3 und 4 der vorliegenden Arbeit dargestellt, geben die Evaluationsergebnisse unterrichtsintegrierter Selbstregulationstrainings Anlass zu einer optimistischen Betrachtung dieses Ansatzes (z.B. Fuchs et al., 2003; Labuhn et al., 2008; Perels et al., 2007; Souvignier \& Mokhlesgerami, 2006). Dennoch bleiben die durch strukturierte Unterrichtsprogramme erzielten Effektstärken oftmals hinter den Erwartungen zurück. Obgleich diesen Interventionen ihre praktische Relevanz keinesfalls abgesprochen werden soll, lassen sie insbesondere im Hinblick auf theoriegeleitete, sozial-kognitiv orientierte Selbstregulationsforschung mit dem Ziel, ein detailliertes Verständnis des Selbstregulationsprozesses in seinen Facetten zu erlangen, viele Fragen offen. Einige dieser Fragen werden im weiteren Verlauf dieser Arbeit aufgegriffen. Ein modifizierter Forschungsansatz soll aufgezeigte Schwachstellen verbessern und einige weitere Antworten liefern, die zu einem umfassenden Verständnis der Ansatzpunkte und Chancen für die Integration selbstregulierten Lernens in den Unterrichtskontext beitragen. Zwei Punkte, die direkt an die Grundannahmen des sozial-kognitiven Verständnisses von Selbstregulation anknüpfen, erhalten dabei besondere Aufmerksamkeit. Der erste Punkt bezieht sich auf eine inhaltliche Aussage der Theorie (vgl. Kapitel 2): Der Kontext, in dem Lernen stattfindet und die Art der Instruktion haben einen entscheidenden Einfluss auf die Entwicklung der Selbstregulationsfähigkeit von Lernenden. Der zweite Punkt bezieht sich auf eine strukturelle Annahme: Selbstreguliertes Lernen stellt einen zyklischen Prozess dar. Dies hat Konsequenzen für die Messung, die entsprechend prozessorientiert und kontextspezifisch erfolgen sollte (vgl. Kapitel 5.1).

Vor diesem Hintergrund wurde in einer zweiten Studie der Versuch unternommen, im Kontext schulischen Lernens einzelne theoretisch relevante, prozessorientierte Mechanismen von Instruktion hinsichtlich ihrer Wirkung auf Selbstregulation und Leistung einer experimentellen Überprüfung zu unterziehen. Zentral war dabei die Frage, wie selbstreguliertes Lernen im regulären Unterricht auf eine andere Art und Weise als durch detalliert geplante 
Trainingsprogramme gefördert werden kann. Dazu wurde nach Ansatzpunkten gesucht, die auf universeller Ebene für die Selbstregulation der Schüler(innen) relevant sind und aus denen unabhängig von der Verfügbarkeit von Trainingsprogrammen selbstregulations- und lernförderliche Bedingungen abgeleitet werden können. Zwei solche Ansatzpunkte, die im Hinblick auf erfolgreicheres Lernen optimiert werden können, wurden dabei exemplarisch herausgegriffen: Bewertungsstandards sowie Feedback und dessen Interpretation. Beide haben zentrale Funktionen in der Selbstreflexionsphase und können den weiteren Verlauf des Selbstregulationsprozesses entscheidend beeinflussen. Im Folgenden wird kurz auf die Bedeutung dieser beiden Faktoren innerhalb des Selbstregulationszyklus und auf ihren Bezug zum Unterrichtskontext eingegangen.

\section{Bewertungsstandards}

Ein zentraler Bestandteil der Selbstreflexionsphase ist die eigene Bewertung der erbrachten Leistung durch den Vergleich des festgestellten Ergebnisses mit einem Ziel oder Bewertungsstandard (Winne \& Hadwin, 1998). Standards werden als die erwartete bzw. gewünschte Qualität oder das Vergleichsniveau der Leistung aufgefasst (English \& English, 1958). Ähnlich wie Ziele werden Standards in der Vorbereitungsphase selbst gesetzt oder von außen vorgegeben, oftmals lediglich implizit. Die Art der Standards hat einen entscheidenden Einfluss auf die Bewertung der eigenen Leistung, die Konsequenzen daraus (z.B. Unzufriedenheit/Zufriedenheit, Selbstwirksamkeitsüberzeugungen, Motivation) und damit auf den Verlauf der nachfolgenden Vorbereitungsphase innerhalb des Selbstregulationsprozesses. Besonders bei schweren Aufgaben erleben Lernende ihr Ergebnis aufgrund zu strenger Vergleichsstandards häufig negativ (Kitsantas \& Zimmerman, 2002; Zimmerman \& Kitsantas, 1999). Kitsantas und Zimmerman (2006) zeigen, dass hohe und absolut formulierte Standards die Sensitivität für kleine Verbesserungen im Lernprozess einschränken können. Im Vergleich zu Personen mit graduellen Standards, bei denen das Lernergebnis nicht dichotom als „Erfolg“ oder „Misserfolg“ klassifiziert wird, schnitten Personen mit absoluten Standards schlechter ab: Sie zeigten weniger Bewusstsein für den eigenen Lernprozess, bewerteten ihre eigenen Resultate negativer und wurden zusätzlich von Personen mit graduellen Standards hinsichtlich der Leistung bei der Aufgabenausführung übertroffen. Für das schulische Lernen und die Gestaltung von Unterricht haben solche Forschungsergebnisse eine entscheidende Bedeutung: Es wäre sinnvoll eine Lernumgebung herzustellen, in der die Tatsache betont wird, dass es sich beim Lernen um einen individuellen Prozess und eine persönliche Entwicklung handelt. Das bedeutet z.B. Schüler(innen) darin zu unterstützen ein Bewusstsein für ei- 
gene kleine Fortschritte und deren Zusammenhang mit Arbeitsweise und Strategiegebrauch aufzubauen.

\section{Feedback}

Die Bewertung des eigenen Lernergebnisses in der Selbstreflexionsphase wird neben den Standards auch durch externes Feedback entscheidend beeinflusst. Forschungsarbeiten zeigen, dass die Fähigkeit zur Selbstregulation des Lernens stark von der Qualität des Feedbacks abhängt (Schunk \& Rice, 1993; Schunk \& Schwartz, 1993; Zimmerman, 1989b, 1995a). Lernende benötigen Informationen, die es ihnen erlauben adaptiv ihre Strategien und Arbeitsweise an die Anforderungen anzupassen. Im Schulkontext wird die Rückmeldung häufig jedoch lediglich in Form von Noten gegeben, die weniger den Lernprozess und den individuellen Vergleich als vielmehr das Lernprodukt und den sozialen Vergleich betonen und im ungünstigen Falle kontraproduktive Reaktionen (z.B. Motivationsverlust, sinkende Selbstwirksamkeitserwartung, Angst) hervorrufen. Entwicklungsverlauf und -geschwindigkeit im Lernprozess können jedoch interindividuell stark variieren, und diese Unterschiede sind in Lehr-Lernsituationen zu berücksichtigen (Bloom, 1985). Aus der Perspektive der Selbstregulationstheorie (Zimmerman, 2000) wäre daher prozessorientiertes Feedback zu bevorzugen, das Schüler(innen) dazu anregt ihre eigene Fähigkeitsentwicklung zu verfolgen und sensibel für den Zusammenhang zwischen Arbeitsweise und Lernergebnis zu werden. Darüber hinaus können durch entsprechendes Feedback auch kleine Fortschritte thematisiert und die Selbstreflexion unterstützt werden. Für den Schulkontext bedeutet dies Bedingungen zu schaffen, die trotz des zumeist gegenwärtigen und nicht ganz zu eliminierenden sozialen Vergleichs zwischen Schüler(inne)n, z.B. hervorgerufen durch Noten, von einer Betonung der individuellen Entwicklung der einzelnen geprägt sind.

Externes Feedback und Bewertungsstandards sind also zwei Faktoren, die den Verlauf des Selbstregulationsprozesses entscheidend beeinflussen können. In der zweiten Studie wird daher die Wirkung dieser Faktoren auf die Selbstregulation und den Fähigkeitserwerb untersucht. Im Rahmen dieser Studie sollen darüber hinaus die methodischen Grenzen der Selbstregulationsmessung mittels eher global formulierter Fragebogenitems überwunden werden. Dies wird durch die Erhebung kontextspezifischer Merkmale der Selbstregulation erreicht, die einen präziseren und valideren Einblick in den Prozess selbstregulierten Lernens gewährt. Für eine solche Analyse eignen sich stark strukturierte, regelhafte und strategiebezogene Aufgaben besonders gut. Aus diesem Grund wurde - im Gegensatz zu den facettenreichen Inhalten 
der Biologie-Unterrichtseinheit in Studie 1, welche ein Verständnis für Zusammenhänge und Konzepte verlangen - in der zweiten Studie der Erwerb einer strategischen mathematischen Fähigkeit als Kontext für die Untersuchung gewählt.

\subsection{Selbstwirksamkeitserwartungen, Metakognition und die Frage der "Kalibrierung"}

In Kapitel 4.4.1 (praktische Grenzen der Unterrichtsintervention) wurde im Rahmen der kritischen Betrachtung des Inhaltsspektrums von Selbstregulationsinterventionen festgestellt, dass sich ein breit angelegter Ansatz für die theoriegeleitete Selbstregulationsforschung weniger gut eignet, wenn es darum geht, spezifische Hypothesen aus dem Selbstregulationsmodell (Zimmerman, 2000) abzuleiten und zu prüfen. Mit dem Ziel, Subprozesse und ihre Zusammenhänge genau zu analysieren, erscheint es sinnvoll, einen Teilaspekt der Selbstregulation einer fokussierten Untersuchung $\mathrm{zu}$ unterziehen. Aus sozial-kognitiver Perspektive spielen Selbstwirksamkeitserwartungen im Lernprozess eine zentrale Rolle. Die Einschätzung einer Person bezüglich ihrer Fähigkeit eine bestimmte Aufgabe erfolgreich zu bewältigen hat entscheidende Konsequenzen für Motivation, Herangehensweise, Anstrengung und Ausdauer bei der Bearbeitung. Selbstwirksamkeitserwartungen haben sich nicht nur als motivational günstig erwiesen, sondern wirken sich auch positiv auf Fähigkeitserwerb und Leistung aus (Pajares, 1996; Zimmerman, 1995a). In einer Reihe von Forschungsarbeiten wurde der Einfluss von Selbstwirksamkeitserwartungen auf Subprozesse aller drei Phasen der Selbstregulation (Vorbereitungsphase, Handlungsphase, Selbstreflexionsphase) nachgewiesen (vgl. Schunk \& Ertmer, 2000; Zimmerman, 1995a, 2000). Sie gehören zu den zentralen Komponenten der Vorbereitungsphase und wirken sich dort z.B. auf Planung und Zielsetzung aus. Sie nehmen nachfolgend Einfluss auf die Prozesse der Handlungsphase, z.B. auf die Auswahl von Strategien. Während des Lernens bestimmen sie entscheidend über das Maß an investierter Anstrengung und die Ausdauer bei der Aufgabenbearbeitung. In der Selbstreflexionsphase beeinflussen die Selbstwirksamkeitserwartungen einer Person die Bewertung des eigenen Lernergebnisses, die Reaktion auf die Selbsteinschätzung und die Konsequenzen für die nachfolgende Vorbereitungsphase. Besonders im Schulkontext werden positive Selbstwirksamkeitserwartungen als Grundvoraussetzung für erfolgreiches Lernen betrachtet. Eine optimistische Einschätzung der eigenen Fähigkeiten wirkt sich in der Regel günstig auf die verschiedenen Subprozesse des Lernens aus, z.B. durch eine motivierte und ausdauernde Auseinandersetzung auch mit schwierigeren Aufgaben (Bandura, 1997). Der Theorie entsprechend sind Selbstwirksamkeitserwartungen für die Motivation und Fähigkeitsentwicklung besonders 
günstig, wenn sie geringfügig über dem tatsächlichen Leistungspotenzial einer Person liegen (Bandura, 1986). An diesem Punkt stellt jedoch Pajares (1996, S. 565) die berechtigte Frage „How much confidence is too much confidence?““. Während eine leichte Überschätzung der eigenen Leistung noch funktional im Sinne von motivationsförderlich sein mag, so hat eine größere Diskrepanz eher negative Folgen. Eine deutliche Überschätzung kann die Lernentwicklung und auf längere Sicht die schulischen Leistungen negativ beeinflussen: Die Fehleinschätzung der eigenen Fähigkeiten kann zu unzureichender Auseinandersetzung mit Lerninhalten (Aufgabenanalyse in der Vorbereitungsphase), mangelnder Anstrengung sowie zu fehlender Bereitschaft führen, adäquate Strategien anzuwenden, ausreichend Zeit zu investieren (Handlungsphase) oder über das eigene Lernverhalten zu reflektieren (Selbstreflexionsphase). Es fehlen ein Bewusstsein für die eigenen Stärken und Schwächen und das Vermögen auf letztere zu reagieren. Gerade dieses metakognitive Wissen bezüglich der eigenen Fähigkeit eine bestimmte Aufgabe erfolgreich bewältigen zu können stellt jedoch einen zentralen Bestandteil selbstregulierten Lernens dar (Zimmerman, 1990). Die Genauigkeit der Selbsteinschätzung, oder die „Kalibrierung“ zwischen eingeschätzter und tatsächlicher Leistung (in der englischsprachigen Literatur sind die Begriffe calibration und calibration accuracy geläufig), steht im Zusammenhang mit effizienterem Lernverhalten, (Horgan, 1990), metakognitiven Fähigkeiten und (Schul-) Leistung (Butler \& Winne, 1995; Chen, 2002; Ewers \& Wood, 1993; Kruger \& Dunning, 1999; Nietfeld \& Schraw, 2002; Pajares \& Graham, 1999; Schraw, Potenza \& Nebelsick-Gullet, 1993). Im Rahmen von Untersuchungen zur Beziehung zwischen der Genauigkeit der Selbsteinschätzung (d.h. der Kalibrierung) und Leistungsvariablen zeigt sich jedoch häufig, dass ein Großteil der Schüler(innen) zur Überschätzung der eigenen Fähigkeiten neigt (Pajares \& Kranzler, 1995; Pajares \& Miller, 1997; Schraw et al., 1993) mit den entsprechenden negativen leistungsbezogenen Konsequenzen. Vor diesem Hintergrund wird die Relevanz der metakognitiven Fähigkeit einer genauen Selbsteinschätzung besonders für den Schulkontext deutlich. Die Förderung einer realistischen Betrachtung eigener Kompetenzen sollte neben der Vermittlung fachbezogener Inhalte ein Ziel von Unterricht darstellen (Brannick, Miles, \& Kisamore, 2005). Angesichts des überwiegenden Anteils von Schüler(inne)n, die zur Überschätzung der eigenen Fähigkeiten neigen, stellt sich die Frage, auf welche Weise dieses Ziel verfolgt werden sollte. Nach Pajares (1996) sollte der Ansatz nicht allein die Reduktion der überoptimistischen Selbstwirksamkeitserwartungen sein, sondern statt dessen die Verbesserung des Prozesses der Selbsteinschätzung beinhalten: Die Förderung metakognitiver Fähigkeiten (z.B. Butler, 1998), insbesondere des Bewusstseins für Aufgabenanforderungen (Aufgabenanalyse) und persönliche Stärken und Schwächen, sollte 
eine größere Kongruenz zwischen Selbstwirksamkeitserwartungen bzw. der Selbsteinschätzung und der tatsächlichen Leistung herstellen (Klassen, 2007). In Kapitel 6 wird im Rahmen der Darstellung von Studie 2 detailliert auf Möglichkeiten eingegangen, durch Merkmale der Instruktion die Genauigkeit der Selbsteinschätzung zu erhöhen. Mit der Kalibrierung wird in dieser Studie ein zentraler Aspekt selbstregulierten Lernens fokussiert, der für den gesamten Selbstregulationsprozess von Bedeutung ist. Die zentrale Funktion dieses Aspekts innerhalb des Modells (Zimmerman, 2000) eröffnet die Möglichkeit Annahmen zu prüfen, die sich auf die zyklische Natur der Selbstregulation beziehen.

\section{Messung der Kalibrierung}

Die Messung der Übereinstimmung zwischen der selbst eingeschätzten Fähigkeit und der tatsächlich erbrachten Leistung erfolgt anhand einer mikroanalytischen Prozedur, die ursprünglich von Bandura (1977) vorgeschlagen und in Forschungsarbeiten vielfach aufgegriffen und weiterentwickelt wurde (vgl. Pajares \& Graham, 1999, Pajares \& Kranzler, 1995; Pajares \& Miller, 1997, Schraw et al., 1993, Yates, 1990; für einen Überblick über Forschung zu Kalibrierung und selbstreguliertem Lernen vgl. Stone, 2000). Ausgangspunkt ist in der Regel eine Aufgabe und die kontextspezifische Erhebung der Selbstwirksamkeitserwartung einer Person bezüglich ihrer Fähigkeit, die Aufgabe erfolgreich zu bewältigen (vgl. Tab. 5.2). Es geht also um eine Vorhersage der eigenen Leistung (Prädiktion). Analog dazu wird auch im Anschluss an die Aufgabenbearbeitung die eingeschätzte Sicherheit bezüglich der richtigen Lösung erfragt (Postdiktion). Die Grundlage der Berechnung der Genauigkeitsindizes bilden also die subjektive Einschätzung (Prädiktion bzw. Postdiktion) und die objektiv festgestellte Punktzahl in der bearbeiteten Aufgabe. Die Bestandteile der Messung korrespondieren damit mit den drei Phasen des Selbstregulationsprozesses (vgl. Abb. 5.1).

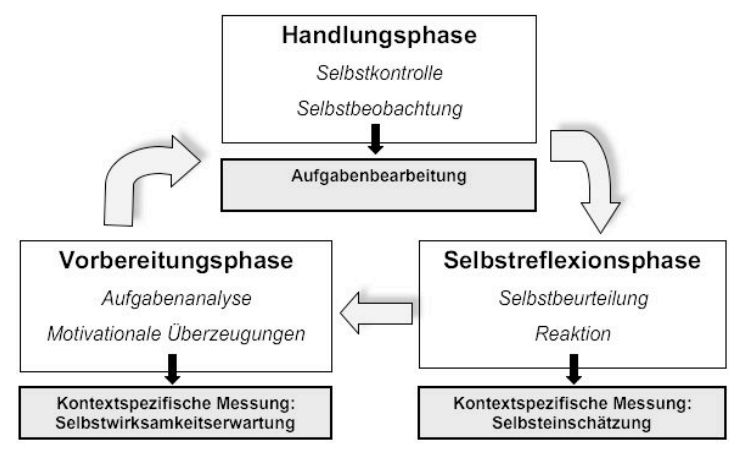

Abbildung 5.1. Kontextspezifische Messung zur Analyse der Einschätzungsgenauigkeit innerhalb des Selbstregulationszyklus 
Die maximal erreichbare Punktzahl und der Wertebereich der Ratingskala sollten ana$\log$ zueinander festgelegt sein (z.B. jeweils 1 bis 9, vgl. Kapitel 6). Aus den festgestellten Werten werden die Verzerrung (Bias; Richtung des Einschätzungsfehlers) und die Genauigkeit (Accuracy; Größe des Einschätzungsfehlers) der Selbstwirksamkeitserwartung bzw. der Selbsteinschätzung berechnet. Für die Berechnung des Bias wird der tatsächlich festgestellte Punktwert in der Aufgabe von der Selbstwirksamkeitserwartung (bzw. der Selbsteinschätzung) subtrahiert. Für eine Person, die sich sehr unsicher ist, die Aufgabe richtig zu lösen (bzw. gelöst zu haben) (Rating von 1), die jedoch die volle Punktzahl erreicht (9), ergibt sich ein Bias von -8 (1-9). Eine Person mit der gleichen Unsicherheit bezüglich ihrer Fähigkeit (1) und tatsächlichem Misserfolg (1) erhält einen Bias von 0 (1-1). Der Wert des Bias reicht also in diesem Beispiel von -8 bis +8 , wobei negative Werte eine Unterschätzung und positive Werte eine Überschätzung der tatsächlichen Leistung anzeigen. Pajares und Graham (1999) führten zusätzlich einen Wert für die Größe des Einschätzungsfehlers (accuracy) ein, für den der Betrag des Bias von 8 (in unserem Beispiel) subtrahiert wird. Accuracy liegt dementsprechend in einem Bereich von 0 bis 8 , mit 0 als Indikator für starke Fehleinschätzung und 8 als Indikator für eine genau mit der tatsächlichen Leistung übereinstimmenden Einschätzung (complete accuracy).

Tabelle 5.2. Messung der Genauigkeit von Selbstwirksamkeitserwartung und Selbsteinschätzung

\begin{tabular}{|c|c|c|c|}
\hline Variable & Item & Berechnung & Wertebereich \\
\hline $\begin{array}{l}\text { Prädiktion: } \\
\text { Selbstwirksamkeitserwartung } \\
\text { (SW) }\end{array}$ & $\begin{array}{l}\text { Wie sicher bist du dir, } \\
\text { dass du diese Aufga- } \\
\text { be richtig lösen } \\
\text { kannst? }\end{array}$ & Ratingskala $0-x^{a}$ & {$[0,1, \ldots, \mathrm{x}]$} \\
\hline SW Bias & & SW - Punktzahl & {$[-(\mathrm{x}-1),-(\mathrm{x}-2), \ldots,(\mathrm{x}-1)]$} \\
\hline SW Accuracy & & $\begin{aligned} & (\mathrm{x}-1)-\mid \mathrm{SW}-\text { Punktzahl } \mid \\
= & (\mathrm{x}-1)-\mid \text { bias } \mid\end{aligned}$ & {$[0,1, \ldots, \mathrm{x}]$} \\
\hline $\begin{array}{l}\text { Postdiktion: } \\
\text { Selbsteinschätzung } \\
\text { (SE) }\end{array}$ & $\begin{array}{l}\text { Wie sicher bist du dir, } \\
\text { dass du diese Aufga- } \\
\text { be richtig gelöst hast? }\end{array}$ & Ratingskala $0-x$ & {$[0,1, \ldots, \mathrm{x}]$} \\
\hline SE Bias & & SE - Punktzahl & {$[-(\mathrm{x}-1),-(\mathrm{x}-2), \ldots,(\mathrm{x}-1)]$} \\
\hline SE Accuracy & & $\begin{aligned} & (\mathrm{x}-1)-\mid \text { SE }- \text { Punktzahl } \mid \\
= & (\mathrm{x}-1)-\mid \text { bias } \mid\end{aligned}$ & {$[0,1, \ldots, \mathrm{x}]$} \\
\hline
\end{tabular}

Anmerkung.

${ }^{a}$ Das Maximum der Ratingskala entspricht der maximal erreichbaren Punktzahl der Aufgabe 


\section{Enhancing students' self-regulation and mathematics performance: The influence of feedback and self-evaluative standards ${ }^{7}$ (Studie 2)}

\subsection{Abstract}

The purpose of this study was to examine the effects of self-evaluative standards and graphed feedback on calibration accuracy and performance in mathematics. Specifically, we explored the influences of mastery learning standards as opposed to social comparison standards as well as of individual feedback as opposed to social comparison feedback. 90 fifth grade students were randomly assigned to experimental and control groups. We conducted analyses for both the complete sample and an at-risk group of low performing students who overstimate their skills. Self-evaluative standards had no effect on calibration accuracy and performance. Students who received feedback were more accurate in their self-evaluative judgements than students who received neither type of feedback. In overconfident students, feedback additionally increased prediction accuracy and performance. Feedback that involved social comparative information tended to be more supportive than individual feedback on several meausures. Educational implications of findings are discussed.

\subsection{Introduction}

International comparative studies such as the Program for International Student Assessment (PISA; OECD 2004) or the Third International Mathematics and Science Study (TIMSS; Mullis, Martin, Gonzalez, \& Chrostowski, 2004) revealed low levels of mathematics achievement in middle-school students in many Western countries. The evident difficulties are not only a matter of concern in the United States but are also transnationally. Educators, policy-makers, and researchers have sought to gain a deeper understanding of what in particular makes students struggle. There is a strong need to find ways to facilitate students' learning and thus enhance their skills. Hereby, one key question is how to design lessons or interventions to accomplish this challenging task.

Researchers suggest that one way to promote the aquisition of knowledge and skills is to help students regulate their learning; that is, to become more metacognitively, motivationally, and behaviorally responsible for their own learning (Zimmerman, 1995a, 1995b, 2000, 2002). According to theoretical approaches (cf. Winne, 2000; Zimmerman, 2000), the use of

\footnotetext{
${ }^{7}$ Dieses Kapitel entspricht: Labuhn, A. S., Zimmerman, B. J. \& Hasselhorn, M. (submitted). Enhancing students' self-regulation and mathematics performance: The influence of feedback and self-evaluative standards.
} 
self-regulation should result in improved performance. Empirical studies have proven the strong link between the capacity to self-regulate one's learning and self-efficacy, intrinsic task interest (Schunk, 1986, 1996; Zimmerman, 1995), and academic achievement (e.g., Pintrich \& DeGroot, 1990; Zimmerman \& Martinez-Pons, 1986, 1988). The ultimate goal therefore is to enhance academic achievement through increasing self-regulation in students. The question remains in what way a clear understanding of self-regulatory processes does contribute to achieving this goal.

Representing a social-cognitive perspective, Zimmerman's (2000) cyclical model of self-regulation provides an appropriate framework for promoting students' learning in an academic context. According to this theory, self-regulated learning is defined by a cycle of three sequential phases: forethought, performance, and self-reflection. The forethought phase refers to processes that precede action. The key self-regulatory categories associated with this phase are task analysis and sources of motivation, such as self-efficacy beliefs. The performance phase involves processes that relate to and occur during action and are grouped into two major categories: self-control and self-observation. Self-control refers to various strategies the learner uses to complete the task, and self-observation includes metacognitive monitoring or self-recording one's performance. The self-reflection phase occurs after engaging in a task and is directly related to the person's performance. The major categories of this phase are self-judgements and self-reactions that are based on those judgements. A key type of selfjudgement is self-evaluation, which refers to comparing the learning outcome with a goal or standard. As a reaction to this comparison, certain affects, such as satisfaction or dissatisfaction, arise. The self-reflection processes, in turn, influence the subsequent self-regulatory cycle, for example, the motivational precondition or the adaption of strategies to succeed on the task (e.g. Zimmerman, 2000, 2002). As depicted by the model, an essential component of self-regulation is a person's metacognitive awareness of his or her own processes of learning or engaging in a certain task (Zimmerman, 1990, 1994). Accordingly, Zimmerman (1990) proposed that self-regulated learners are aware of whether they do or do not know something. This metacognitive skill is closely related to a concept known as calibration or calibration accuracy (e.g., Pajares \& Kranzler, 1995; Shraw, Potenza \& Nebelsick-Gullet, 1993; Stone, 2000; for the related concept of accuracy in metamemory research, see Hager \& Hasselhorn, 1992; Hasselhorn \& Hager, 1989). It refers to the degree to which students' judgements about their capability or performance actually represent their competence (Horgan, 1990; Pajares \& Kranzler, 1995). Evidence shows that calibration accuracy is related to more efficient study habits (Horgan, 1990), to metacognitive skills as well as to achievement (Butler \& Winne, 
1995; Chen, 2002; Ewers \& Wood, 1993; Kruger \& Dunning, 1999; Nietfeld \& Shraw, 2002; Pajares \& Graham, 1999; Schraw et al., 1993). In prior research, calibration has often been assessed by comparing students' judgements about their perceived capability to complete the task successfully (that is, their task-specific self-efficacy) with their achieved score (e.g., Chen, 2002; Pajares \& Graham, 1999; Pajares \& Kranzler, 1995; Pajares \& Miller, 1994, 1997). Some researchers also used postdictions as a measure to calculate calibration accuracy (Schraw et al., 1993; Zimmerman \& Ramdass, submitted). Like predictions, students' selfevaluative judgements after completing a task are compared with their task performance. These postdictions can provide additional information about a student's performance monitoring and adjustment (Butler, 1998). Once students have finished a task, they have a more complete knowledge of the accuracy of their judgements (Hacker, Bol \& Horgan, 2000; Stone, 2000). In their study about the effects of a revision strategy training, Zimmerman \& Ramdass (submitted) found a larger improvement of postdiction accuracy as compared to prediction accuracy after training. It seems plausible that calibration of self-evaluative judgements (after performance) is more sensitive to interventions that intend to improve self-monitoring because it allows students to refer to their experience with the task (Bol, Hacker, Shea, \& Allen, 2005). Also vice versa, self-evaluative accuracy is critical for subsequent self-regulation according to the cyclical model of self-regulation.

\section{Calibration within a cyclical model of self-regulation}

In terms of the cyclical model of self-regulation mentioned above, calibration of both self-efficacy (before completing the task) and self-evaluation (after completing the task) should result from an efficient interplay of a) a precise task analysis based on knowledge about the own competencies during forethought (Borkowski, 1992; Chen \& Zimmerman, 2007; Pajares, 1996), b) self-monitoring during performance (Schraw et al., 1993; Stone, 2000), and c) self-judgements that are close to actual performance during self-reflection. As Bandura (1986) proposes, being able to make accurate judgements of one's capability is pivotal for successful academic achievement. Hence, students will only be able to self-regulate their studying effectively if they monitor and self-evaluate their progress accurately and thus make adaptions that are based on a correct analysis of their performance. However, most empirical studies of performance calibration reveal that the vast majority of students lack skills to estimate their performance accurately: Findings suggest that many students are overconfident about their capability to perform on a certain task (Pajares \& Kranzler, 1995; Pajares \& Miller, 1997), and that overconfidence is often related to lower performance levels (e.g. Bol 
\& Hacker, 2001; Bol et al., 2005; Chen, 2002; Ewers \& Wood, 1993; Hacker, Bol, Horgan \& Rakow, 2000; Klassen, 2006; Kruger \& Dunning, 1999; Pajares \& Graham, 1999; Pajares \& Kranzler, 1995; Winne \& Jamieson-Noel, 2002). From a self-regulation perspective, miscalibration is a severe problem because students' own view of how they study is critical in selfregulated learning: Students regulate learning in relation to their interpretation of their behavior and its effects (Winne \& Jamieson-Noel, 2002; Winne, 1997). Those who overestimate their performance are likely not to spend the adequate effort to accomplish a task, they may neither engage in effective comprehension monitoring nor deploy appropriate strategies. Moreover, there may be unwillingness to change their study methods (Zimmerman et al., 1996). This is reflected by the fact mentioned above that overestimation is often related to poor performance. Thus, there is a strong need to develop instructional techniques and intervention strategies to improve calibration, especially in those at-risk-students who tend to grossly overestimate their capabilities.

\section{Improving calibration}

One possible way to improve calibration is feedback, which is an inherent component of self-regulated learning (Butler \& Winne, 1995). Feedback from an external source (e.g., teachers, peers) provides learners with information about how well they are performing (Butler \& Winne, 1995) and can influence how the task is re-assessed and how progress is monitored (Stone, 2000). Outcome feedback is usually external feedback, based on performance measures (Stone, 2000). Although outcome feedback does not inform students about how to self-regulate, it may make them more aware of the quality of their achievement, motivate them to monitor their performance in the course of action (Butler \& Winne, 1995), and may have an influence on students' confidence about their capability (Stone, 2000). According to the cyclical model of self-regulation (Zimmerman, 2000), feedback enhances self-reflection, which in turn should result in self-evaluative judgements that are close to actual performance (calibration). In addition, since self-reflection influences the subsequent forethought phase, feedback can affect how students prepare for future tasks and lead to increased selfmonitoring during performance. As Winne (1997) states, learners need practice with feedback: In order to enable students to use feedback efficiently for self-regulation, it should be provided continously on learning tasks (Zimmerman, 1990). However, in an experimental study by Schraw et al. (1993), students received outcome feedback on their reading comprehension and math performance, which turned out not to show any effects on test achievement or calibration accuracy. The authors attribute this result to the fact that the type of outcome 
feedback they used (correct solutions after each block of questions) did not support calibration or self-regulation appropriately. Hacker et al. (2000) provided students with feedback on their performance over multiple tests. Only high-achieving students benefited from the external feedback and increased their predictive and especially postdictive accuracy. Obviously, students must have enough knowledge to use feedback effectively (Stone, 2000). A certain degree of task-specific and metacognitive knowledge (e.g., about the own strengths and weaknesses) is a key prerequisite that allows one to make adaptions in order to meet the requirements of a task. Hence, low-performing students may be considered an at-risk population. This seems to be true for reasons both poor academic achievement as well as miscalibration and the fact that they benefit less from feedback interventions.

Taken together, it seems that calibration accuracy and academic achievement are not easy to improve by mere outcome feedback, especially in low-performing students. One possible explanation for this lack of effects could be that outcome feedback does not sufficiently promote self-monitoring because it does not focus on the learning progress (Stone, 2000). Consequently, researchers have suggested other types of feedback (e.g., process feedback) that involve more than only binary information about whether the results are correct or incorrect. Unfortunately, in many educational settings, more preferable types of feedback are hard to provide due to a lack of time, staff, or other aspects that hinder instructors to focus on details in each student's performance. As Schraw et al. (1993) emphasize, educators should look for ways to promote self-control such as encouraging students to monitor their selfimprovement over time and reflect on reasons for their improvement. How can this be accomplished given the fact that outcome feedback is the most common type of feedback in the classroom context? One possible way to combine the idea of mere outcome feedback with supporting students to focus more on the process of learning could be the method of graphing. Recording a person's outcome over several tasks or trials and presenting the information by visual graphs can enhance the use of a personal comparison standard (Bandura, 1986) and should support monitoring as well as self-reflective processes. In a study by Kitsantas and Zimmerman (2006), students who graphed their learning outcomes showed significantly greater awareness of learning progress and even a significant improvement in actual learning. Hence, presented as a type of feedback that goes beyond mere outcome feedback, graphing appears to be a powerful support of students' self-regulated learning. 


\section{Feedback and social comparison}

Speaking of performance feedback that is related to academic achievement and usually occurs in a classroom context, one has to bear in mind that there is always a certain degree of social comparison present in such an environment. As an almost inevitable element of social interaction, social comparison may occur automatically (Brickman \& Bulman, 1977). That is, in the classroom it is most likely that students are aware of the approximate achievement levels of their classmates and how well others perform compared to them. From a self-regulation perspective, one might assume that graphing an individual's results over several trials could be most beneficial for self-monitoring if it was truly independent of any social comparison information. Presented this way, it would solely focus on the personal development of skill. However, while the method of graphing may be implemented into daily activities in the classroom, it appears to be unrealistic to eliminate social comparative influences. Does that mean that it is impossible to create optimal conditions for supporting students' self-regulated learning in a classroom context? Furthermore, are students distracted from their individual progress when learning in a group? As France-Kaatrude and Smith (1985) state, social comparison in the classroom does not necessarily have detrimental effects. To the extent that social comparison information permits meaningful self-evaluation of the own performance, it may even enhance task motivation. This is in line with Festinger's (1954) social comparison theory postulating that people usually strive to evaluate their opinions and abilities, and that one way to self-evaluate is to compare oneself with others. Still, other research indicates negative effects of social comparison in the classroom context, especially for low performing students (Thompson \& Musket, 2005; Thompson \& Perry, 2005). In accordance, Bandura and Jourden (1991) reported that social comparative influences affect self-regulatory factors governing motivation and performance: In their study with business students, those who assumed to constantly perform worse than another student showed a drop in their self-efficacy beliefs and performance. Therefore, it remains an important question how social comparison information (like in classrooms) affects learners' self-regulation. It is of particular interest how it influences the effects of the type of graphing feedback we have introduced above. 


\section{Interpreting feedback: The role of self-evaluative standards}

Having stressed that feedback is assumed to support cognitive and metacognitive processes and hence calibration accuracy, it is important to note that any kind of external feedback will not have any effects on learners' behavior until they process and interpret the information provided. The self-reflection phase as derived from Zimmerman's model (2000) involves comparing one's learning outcomes with a previously set standard, which is defined as the expected or preferred quality or level of performance (English \& English, 1958). Like goals, standards can be set personally or externally during forethought (Kitsantas \& Zimmerman, 2006) and used for self-evaluation in the self-reflection phase. The learner undertakes a kind of target-performance comparison, which is another key process within the cycle of selfregulation: It influences the learner's self-judgement, which in turn affects the level of selfsatisfaction and, if necessary, the readiness to adapt the procedure (e.g., the applied strategy) to the requirements of the task. For externally set standards, Kitsantas and Zimmerman (2006) report that graduated, as opposed to absolute standards (e.g., classification of outcomes as either successes or failures), enhance students' awareness of learning progress and thus their sensitivity to small improvements in skill. Students who used a graduated standard showed higher levels of skill, self-efficacy and self-satisfaction. In other words, graduated standards place great value on progressive mastery of a task.

If standards not only involve subtle gradiations in performance, but also appreciate each person's individual development of skill, we speak of mastery learning standards.

By emphasizing the individual progress of learning, mastery learning standards should advantageously support the interpretation of feedback that is provided over several trials. This might especially be true for graphing feedback since it explicitly mirrors the learning progress. However, in a classroom context where social comparison is often present (either implicitly or explicitly), mastery learning standards can be difficult to implement. The fact that students are compared to each other and are aware of this comparison seems to be true for many educational contexts. If standards are set by social comparison, this could distract the student from focussing on the own progress in a longitudinal manner by placing emphasis on the cross-sectional comparison with others. 


\section{Current study}

To our knowledge, there are yet no experimental studies that investigate a) the effects of individual as opposed to social comparison feedback that is presented as a graph over several trials on students' calibration accuracy and mathematics performance, b) likewise, the effects of mastery learning as opposed to social comparison standards, and c) whether students benefit differently from feedback depending on the self-evaluative standard they employ.

Therefore, the present study was designed to establish the hypothesized connection between key self-regulatory processes and the acquisition and improvement of a new math skill. It was intended to explore the effects of two types of graphing feedback (individual vs. social comparative), provided continuously over several learning trials, on students' calibration accuracy and achievement in a new math task. It is of particular interest if the positive effect of graphing on performance and self-regulation reported by Kitsantas and Zimmerman (2006) can be found even in the presence of social comparison. This would make the method of graphing highly recommendable for the classroom context. The current study also attempts to examine the impact of two types of self-evaluative standards (mastery vs. social comparison) as well as the combined effectiveness, that is, the interplay of feedback and standards. It was predicted that mastery learning standards would instill an increased focus on the individual process of learning and additionally support the effect of graphing feedback. Thus, the effects of mastery learning standards and individual feedback were expected to be additive.

In a first step, we intend to explore the effects for the entire sample. In a second step, we will focus only on the at-risk group of miscalibrated students who overestimate their performance. The rationale for this two-step process of analysis is that prior research has shown that the majority of students tend to be overconfident (Pajares \& Kranzler, 1995; Pajares \& Miller, 1997), and that overconfidence covaries with lower levels of achievement (e.g. Bol \& Hacker, 2001; Bol et al., 2005; Chen, 2002; Ewers \& Wood, 1993; Hacker et al., 2000; Klassen, 2006; Kruger \& Dunning, 1999; Pajares \& Graham, 1999; Pajares \& Kranzler, 1995; Winne \& Jamieson-Noel, 2002). Consequently, there is a strong demand to design instruction in order to meet the needs of this group. New ways have to be found to enhance both calibration accuracy and performance in overconfident students. 


\subsection{Method}

\section{Participants}

The participants in this study were 90 fifth grade students ( 37 boys and 53 girls) from a German grammar school (Gymnasium) ${ }^{8}$. Their mean age was 10 years; 3 months $(S D=6$ months). Students came from predominantly middle-class-families and their first language was German. Participation in the study was voluntary and required parental permission.

\section{Task Materials}

The mathematical task chosen for this study includes a combination of multiplication, addition, subtraction, and parantheses (see Table 1). There are two mathematical rules to be followed in order to solve the task correctly: a) the term in parantheses has to be solved first and b) multiplication and division has to be done before addition and subtraction. Althought the students in our study had all the prerequisite mathematical skills to solve the problems, they had at least only briefly heard about the two rules before, and the combination of them within one problem was rather unfamiliar to them. According to the German national curriculum for mathematics, this type of task is scheduled for the middle or end of fifth grade. Since the experiment took place right at the beginning of the semester, students had hardly prior experience in the solving these problems. According to the results of a pilot study, we chose rather easy tasks for the pretest (in order not to overstrain students at the beginning of the experiment) and equally balanced task difficulty between practice phase and posttest.

Table 6.1. Math task examples

$$
\begin{aligned}
& (51+37)-9 * 6 \\
& 8 * 7+5+(43+29) \\
& 6 * 7+(86-37) \\
& 3+(91-55)+3 * 9 \\
& (36+28)-8 * 4-3
\end{aligned}
$$

\footnotetext{
8 In the German school system, after attending primary school, students are assigned to one of three school tracks, according to their academic achievement: Hauptschule (lower achievement level), Realschule (middle achievement level) and Gymnasium (higher achievement level).
} 


\section{Design and Procedure}

The students participated individually in a single session that lasted approximately forty minutes. The sessions took place in a quiet room at the high school and were conducted by one of two research assistants who had received extensive training before. Participants were randomly assigned to one of nine groups. The experimental conditions were based on three types of self-evaluative standards (mastery learning, social comparison, without any standards) and three types of feedback (individual, social comparative, without any feedback). There were ten students in each group. For the present sample size, the statistical power for planned $F$-tests for detecting a large effect is 0.93 (Cohen, 1988).

At the onset of the experiment, students were given a brief description of the procedure and asked whether they were willing to participate (no one refused to participate). Each session consisted of four phases: 1) an instruction phase, 2) a pretest phase, 3) a practice phase, and 4) a posttest phase. In the instruction phase, students received identical instructions about the task and the required three-step problem solving strategy regardless of the experimental group they were in: 1) First do all operations that lie inside parentheses, 2) do all multiplication and division before addition and subtraction, and 3) Finally, after following step one and two, work from left to right, do all addition and subtraction. Hereby, the experimenter demonstrated how to apply the strategy step by step by giving two examples. Then students were pretested for problem solving performance and self-evaluation after problem solving. The following phase (practice) was critical for the experimental procedure. At the beginning, the students received a short introduction according to the experimental condition (see Appendix B for instructions; students in any feedback conditions were shown the graph and given a brief explanation in order to make sure that they understood how the graphing was supposed to work). During practice phase, they received four sheets altogether with five mathematical problems on each sheet (see Appendix D). The experimental groups were designed as follows:

Students in group 1 (control) did neither get any information about standards nor any feedback after having solved the problems on a sheet. The experimenter just told them that when they were done with the first sheet, they would get the next one and so forth. Students assigned to group 2 (mastery learning standards without feedback) were given mastery learning standards information. The wording was as follows: 'Everyone can succeed. One can learn to perform well at this task. It is great if you do the best that you can and try to improve your own skills step by step.' They did not get any feedback during the practice phase. Par- 
ticipants in group 3 (social comparison standards without feedback), were told the following: 'Students perform differently at this task. Some do very well at it, others don't do so well. We will see how well you can do it.' They did not get any feedback either. In contrast, students in group 4 (mastery learning standards and individual feedback) were given the same mastery learning standards information as group 2, and additionally received individual feedback on each sheet completed during practice phase. The experimenter filled in the score on a graph that was placed next to the table. There was a maximum score of five points ( 5 problems) for each of the four sheets. Students in group 5 (social comparison standards and individual feed$\underline{\text { back) }}$ got the same social comparison standards as did group 3. They received individual feedback. Participants assigned to group 6 (no standards and individual feedback) only received individual feedback during practice phase without having been given any standards information before. Students in group 7 (mastery learning standards and social comparative feedback) were given mastery learning standards information. They also received feedback, and their score was filled in on a graph, too. Additionally, each time they received feedback, the experimenter told them 'how many points most of the other students have earned' and filled in their score on the graph using another colour. The sessions of the three experimental conditions that contained social comparative feedback took place after the other six groups were done in order to obtain the mean scores for each sheet. Thus it was possible to give the participants in the social comparative feedback groups ecologically valid comparative information that was based on the performance of the other students in the experiment. Students in group 8 (social comparison standards and social comparative feedback), who were given social comparison standards information before, received the same social comparative feedback during practice phase. Finally, participants in group 9 (no standards and social comparative feedback) received social comparative feedback, too, without having been given any standards information before. After the practice phase, students were posttested for self-efficacy, problem solving performance, self-evaluation, attribution, self-appraisal, and self-satisfaction.

\section{Measures}

Demographic information. At the beginning of the session, students completed a demographic sheet to obtain information about their age, gender, nationality, first language, favorite subject, and math grade on their previous report card.

Mathematical problem solving performance. Independently from students' math performance during the practice phase of the experiment, we measured problem solving performance by eight math problems in both pretest and posttest. The solution of each problem 
was coded either right or wrong resulting in a maximum score of 8 points (see Appendix $\mathrm{C}$ for all measures)

Self-efficacy beliefs. Before solving the eight problems on the pretest and posttest respectively, students were asked to look over them and indicate how confident they were that they could solve these problems correctly. They marked their judgement on a 9 point scale ranging from $1=$ 'definitely not confident' to $9=$ 'extremely confident.'

Self-evaluation. After attempting the 8 math problems we measured students' perceived effectiveness of their solutions. The wording was as follows: 'How confident are you that you solved these problems correctly?' We used the same rating scale as for the selfefficacy scale above, namely 1 = 'definitely not confident' to 9 = 'extremely confident.'

Self-judgement. To assess their self-evaluative judgements, we asked students two consecutive questions. The wording of the first item was 'How do you evaluate your problem solving performance?,' and the 9 point rating scale ranged from $1=$ 'not good at all' to $9=$ 'very good.' This was followed by an open-ended response question, 'Why do you evaluate your performance that way?.' Answers were recorded verbatim. According to Kitsantas and Zimmerman's (2002) measure, two independent raters classified each student as either a 'selfevaluator' or a 'non-self-evaluator.' The rating was made based upon how reasonable student's examples for self-evaluation were. Cohen's Kappa analyses revealed that inter-rater agreement for the classification was perfect (1.00).

Self-satisfaction. To measure how satisfied students were with performance during the posttest they were asked: 'How satisfied are you with your problem solving performance?'. The 9 point scale ranged from $1=$ 'very dissatisfied' to $9=$ 'extremely satisfied.'

Calibration of math self-efficacy and self-evaluation. We computed bias (direction of judgment errors) and accuracy (magnitude of judgment errors) measures of self-efficacy and self-evaluation respectively. We based the calculations on procedures suggested by Pajares and Graham (1999), Pajares and Miller (1997), Schraw et al. (1993), and Yates (1990), and successfully applied by Chen and Zimmerman (2007) and Zimmerman and Ramdass (submitted). In order to compute bias, we scored problem solving performance from 1 (0 problems solved correctly) to 9 ( 8 problems solved correctly) corresponding to the self-efficacy and self-evaluation judgements ranging from 1-9. Then we subtracted the problem solving score (1-9) from either the self-efficacy or the self-evaluation score. Thus, a student who expressed 'not at all confident' (1) in solving the problems correctly and missed all problems (1) received a bias score of 0 (1-1). Alternately, a student with the same lack of confidence who correctly solved three problems received a bias score of -3 (1-4), indicating underconfidence. 
Therefore, self-efficacy and self-evaluation calibration bias scores ranged from -8 to +8 . To compute calibration accuracy, we subtracted the absolute value of each bias score from 8 , indicating the magnitude of the judgment error, with a range of 0 (complete inaccuracy) to 8 (complete accuracy).

\subsection{Results}

The effectiveness of random assignment procedures in distributing girls and boys equally in each experimental group was compared using chi -square procedures. No statistically significant differences emerged. Statistical analyses of the pretest data also revealed no significant gender differences in math problem solving performance and self-evaluation measures. As a result of this initial comparability, the data from boys and girls were combined for all subsequent analyses.

The impact of feedback (individual, social comparative, none) and self-evaluative standards (mastery, social comparative, none) on calibration and performance was analyzed using a $3 \times 3$ analysis of variance (ANOVA). Chi-square procedures were used to assess differences for categorical data (verbal self-judgements). Finally, zero-order correlations between the dependent measures were performed. Means and standard deviations for all dependent measures are presented in Table 2.

Analyses for the Complete Sample.

Calibration. We did not find any effects for the posttest measures of self-efficacy accuracy and bias. However, looking at students' judgements after completing the task, there was a significant main effect of feedback for both self-evaluative accuracy $(F(2,81)=5.310$, $p<.01$, eta $\left.^{2}=.116\right)$ and self-evaluation bias $\left(F(2,81)=5.032, p<.01\right.$, eta $\left.^{2}=.111\right)$. Post-hoc Tukey tests revealed that students who received either social comparison feedback $(M=6.67$, $S D=1.30)$ or individual feedback $(M=6.37, S D=1.67)$ showed significantly higher accuracy than students in the control group $(M=5.33, S D=2.02)$. Also, these groups were significantly less biased than the control group (social comparison: $M=0.87, S D=1.66$, individual: $M=0.83, S D=2.20$, control: $M=2.33, S D=2.41)$. There was neither significant main effect for self-evaluative standards nor an interaction between feedback and selfevaluative standards.

Self-judgement. The two self-judgement categories (self-evaluator vs. no selfevaluator) were compared based on the self-evaluative standard set before the practice phase 
(mastery, social comparison, or none) in a $2 \times 3$ chi-square analysis. No significant differences were found. When we applied the same analysis to the three feedback groups (individual, social comparison, or none), it revealed a significant difference between these groups in their capability to give a reasonable explanation for their judgement about their own performance quality, chi $^{2}(2)=7.569, p<.05$; Cramer's $V=.290$. More students who received feedback (either individual or social comparative) were classified as self-evaluators than students in the control group.

Satisfaction. For the measure of satisfaction after performance, the analysis revealed a significant main effect of feedback $\left(F(2,81)=3.270, p<.05\right.$, eta $\left.^{2}=.075\right)$. Post hoc Tukey tests indicated that students in the individual feedback group were most dissatisfied with their performance $(M=4.70, S D=1.92)$. Both students in the social comparison $(M=5.80$, $S D=1.94)$ and control group $(M=5.73, S D=1.66)$ reported significantly higher satisfaction. There was no significant main effect for self-evaluative standards or interaction between feedback and self-evaluative standards.

Mathematics problem solving performance. In terms of the posttest measure of mathematical problem solving, the analysis yielded no significant main effects for feedback, standards, or the interaction between these factors. 
尚

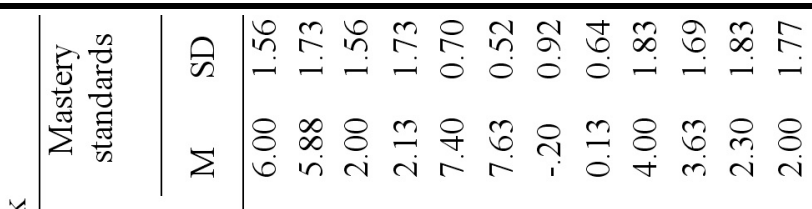

$\frac{4}{8}$

总

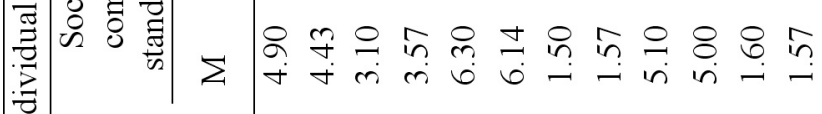

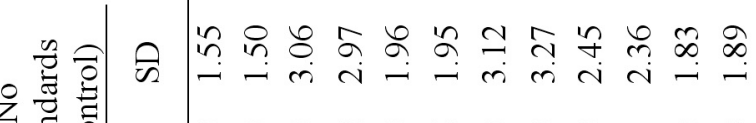

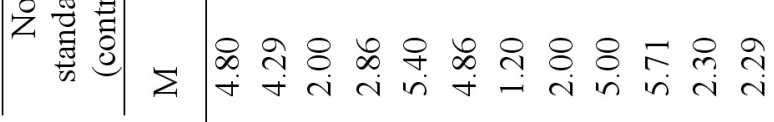

言

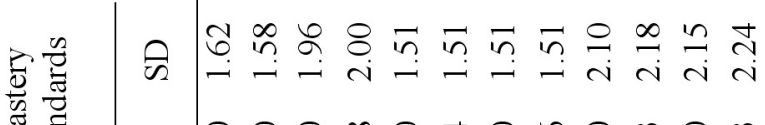

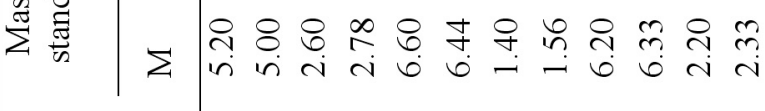

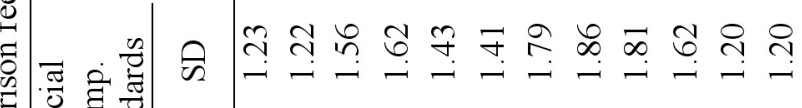

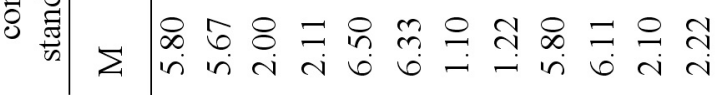

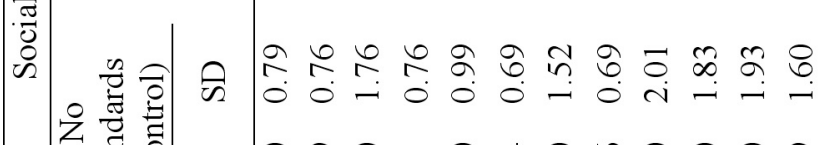

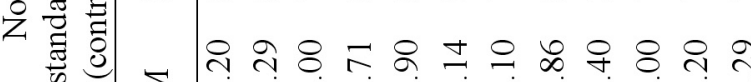

क्ष

ڤี

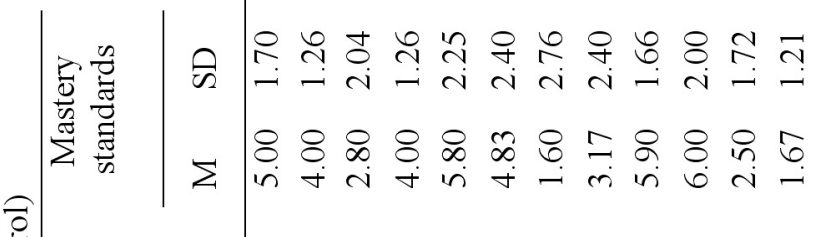

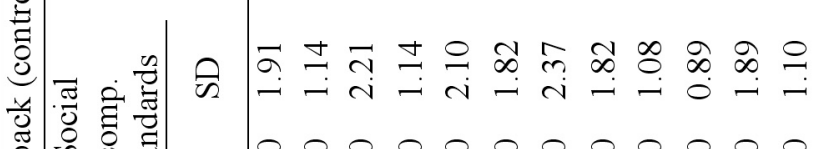

焉

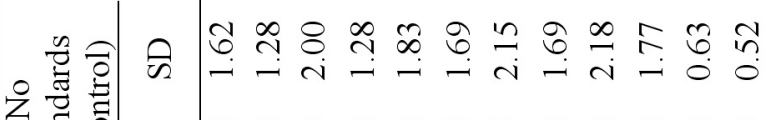

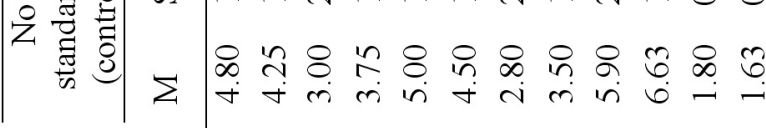

$\sum$

苂

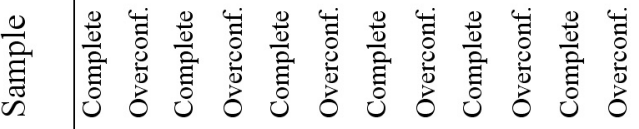

อั๊

กั่

$\frac{\sqrt{0}}{\frac{0}{0}}$

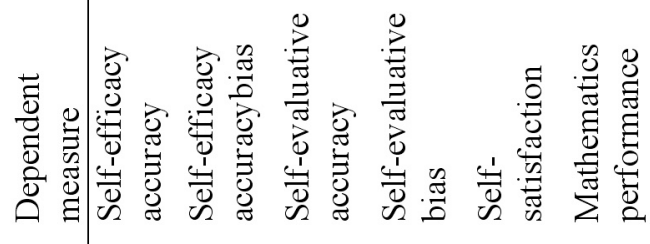




\section{At-risk Group Analyses}

We also tested our hypotheses for a subgroup of students who were at greater risk. To identify this group, we used the pretest measure of self-evaluative bias in order to determine students' level of calibration. Based on this measure of postdiction accuracy, we defined the at-risk group we focussed on in the second step of analyses. Those who overestimated their performance and thus showed a positive bias score (indicating that self-evaluation was higher than actual performance) were labeled overconfident. Chi-square procedures revealed that direction of bias (i.e., overconfident, accurate, and underconfident) was equally balanced over the experimental conditions. In line with prior research on calibration (e.g., Pajares \& Kranzler, 1995), the majority of students in our sample were classified as overconfident (overconfident: $\mathrm{n}=66$, underconfident: $\mathrm{n}=12$, accurate: $\mathrm{n}=12$ ). Moreover, an exploratory analysis indicated that these were outperformed by the combined group of accurate and underconfident students on the pretest measure of mathematical problem solving, $F(1,88)=14.86, p<.001$, eta $^{2}=.144(M=2.44, S D=1.56$ vs. $M=3.88, S D=1.57)$. This emphasizes that the overconfident students can be reasonably called a population at risk. The means and standard deviations for the dependent measures in the group of overconfident students are also presented in Table 2. Once again, we did not find any significant main effects of self-evaluative standards for any dependent measures. In the following, we will therefore only report significant effects of feedback and interactions between both factors.

Calibration. The analyses revealed a significant main effect of feedback for both selfefficacy accuracy $\left(F(2,57)=7.601, p<.01\right.$, eta $\left.^{2}=.211\right)$ and bias $(F(2,57)=5.927, p<.01$, eta $\left.^{2}=.172\right)$. Post hoc Tukey tests indicated that students in the social comparison feedback group $(M=5.60, S D=1.32)$ showed significantly higher self-efficacy accuracy than students in the control group $(M=4.00, S D=1.20)$ and that both feedback groups were less biased than the control group (social comparison: $\mathrm{M}=2.24, \mathrm{SD}=1.59$, individual: $M=2.82$, $S D=2.20$; control: $M=4.00, S D=1.20)$. No interaction between feedback and selfevaluative standards emerged for these measures. For self-evaluative accuracy and bias there were also significant main effects for feedback $\left(F(2,57)=12.12, p<.001\right.$, eta $^{2}=.298$ and $F(2,57)=11.11, p<.001$ eta $\left.^{2}=.280\right)$. Post hoc Tukey tests revealed that students in both feedback groups were significantly more accurate in their self-evaluative judgements (social comparison: $M=6.60, S D=1.29$; individual: $M=6.27, S D=1.88$ ) and showed less bias (social comparison: $M=1.24, S D=1.45$; individual: $M=1.18, S D=2.28$ ) than the control group $(M=4.37, S D=1.92 ; M=3.63, S D=1.92)$. 
For self-evaluative accuracy, there was also a significant interaction between standards and feedback $\left(F(4,57)=2.560, p<.05\right.$ eta $\left.^{2}=.152\right)$. Feedback affected the accuracy of selfevaluative judgments differently depending on the type of self-evaluative standards set before practice. Specifically, while both feedback groups outperformed the control group in all standards conditions, self-evaluative accuracy was higher in students who received social comparison feedback $(M=7.14, S D=0.69)$ than in students who received individual feedback $(M=4.86, S D=1.95)$ when no standards were set; self-evaluative accuracy was similar in students who received social comparison feedback $(M=6.33, S D=1.41)$ and individual feedback $(M=6.14, S D=1.86)$ when social comparison standards where set. Finally, when mastery standards where employed, self-evaluative accuracy was higher in students who received individual feedback $(M=7.63, S D=0.52)$ than in students who received social comparison feedback $(M=6.44, S D=1.51)$.

Self-judgement. As described above, the two self-judgement categories (self-evaluator vs. no self-evaluator) were compared based on the type of feedback (individual, social comparison, or none) in a $2 \times 3$ chi-square analysis. There was a significant difference between the feedback groups in their capability to give a reasonable explanation for their self-judgment, $\operatorname{chi}^{2}(2)=7.252, p<.05$; Cramer's $V=.331$. More at-risk students who received feedback (either individual or social comparative) were classified as self-evaluators than students in the control group.

Satisfaction. For the measure of satisfaction, analyses revealed the same pattern of results as obtained in the complete sample. There was a main effect of feedback $(F(2,57)=$ $3.875, p<.05$, eta $^{2}=.120$ ), and post hoc Tukey tests showed that students in the individual feedback group were most dissatisfied with their performance $(M=4.73, S D=2.00)$. Both students in the social comparison $(M=6.16, S D=1.82)$ and control group $(M=6.16$, $S D=1.64)$ reported significantly higher satisfaction. No interaction was found between feedback and self-evaluative standards.

Mathematics problem solving performance. As opposed to the complete sample, the analysis did reveal a main effect of feedback in overconfident students $(F(2,57)=3.538$, $p<.05$ eta $\left.^{2}=.110\right)$. As post hoc Tukey tests revealed, students who received social comparison feedback scored significantly higher on the posttest measure of mathematical problem solving $(M=2.56, S D=1.73)$ than students in the control group $(M=1.42, S D=0.96)$. There was no interaction between feedback and self-evaluative standards.

In sum, the analyses for the selected group of overconfident students revealed even more obvious effects of feedback than did the analyses for the complete sample. The effect 
sizes obtained in the at-risk group turned out to be larger, and significant effects emerged on all dependent measures, including the performance measure.

\section{Correlation analyses}

Zero-order correlations among the dependent measures for both the complete sample and the group of overconfident students are presented in Table 3. All calibration measures correlated significantly with problem solving performance. Both self-efficacy bias and selfevaluation bias measures showed negative correlations with performance that were remarkably high, indicating that less bias covaried with a higher problem solving score.

Table 6.3. Correlations Among Dependent Measures

\begin{tabular}{lcccccc}
\hline \multicolumn{1}{c}{ Measure } & 1 & 2 & 3 & 4 & 5 & 6 \\
\hline 1. Self-efficacy accuracy & & $-.82^{* *}$ & $.65^{* *}$ & $-.62^{* *}$ & -.15 & $.44^{* *}$ \\
2. Self-efficacy accuracy bias & $-.90^{* *}$ & & $-.43^{* *}$ & $.75^{* *}$ & .13 & $-.59^{* *}$ \\
3. Self-evaluative accuracy & $.65^{* *}$ & $-.51^{* *}$ & & $-.74^{* *}$ & -.15 & $.20^{+}$ \\
4. Self-evaluative bias & $-.63^{* *}$ & $.71^{* *}$ & $-.85^{* *}$ & & .17 & $-.45^{* *}$ \\
5. Self-satisfaction & -.09 & .06 & -.09 & .10 & $.35^{* *}$ \\
6. Mathematics performance & $.50^{* *}$ & $-.60^{* *}$ & $.25^{*}$ & $-.40^{* *}$ & $.33^{* *}$ & \\
\hline
\end{tabular}

\section{Note.}

Correlations above the matrix are for the complete sample $(\mathrm{N}=90)$;

correlations below the matrix are for overconfident students only $(\mathrm{N}=66)$.

${ }^{+} \mathrm{p}<0.10, * \mathrm{p}<0.05, * * \mathrm{p}<0.01$

\subsection{Discussion}

The present study was designed to determine the effects of self-evaluative standards and feedback on fifth-grade students' mathematical performance and calibration accuracy, the latter representing an important aspect of self-regulated learning. Hypotheses were tested both for the complete sample and for a selected group of at-risk students who tend to overestimate their performance. Consistent with prior research, the majority of students in our study were overconfident about their capability to solve math problems (e.g. Bol \& Hacker, 2001; Pajares \& Kranzler, 1995; Pajares \& Miller, 1997), and overconfidence was associated with a lower level of achievement (e.g. Bol \& Hacker, 2001; Bol et al., 2005; Chen, 2002; Ewers \& Wood, 1993; Hacker et al., 2000; Klassen, 2006; Kruger \& Dunning, 1999; Pajares \& Graham, 1999; Pajares \& Kranzler, 1995; Winne \& Jamieson-Noel, 2002). Also, as Pajares and Kranzler (1995) found, there were no significant gender differences in calibration accuracy or bias.

It was hypothesized that setting mastery learning standards can increase a learner' $\mathrm{s}$ focus on his or her personal development of skill. Contrary to our hypothesis we found that in the complete sample different forms of self-evaluative standards had no significant impact on 
accuracy scores, bias, or problem solving performance. Clearly, it made no difference what type of standard was set externally (i.e., by the experimenter) before the practice phase of the experiment. One possible explanation for this result is that the experimental manipulation was just not powerful enough. Students might not have related the brief information about standards provided in the early phase of the experiment to their own performance during the practice phase. Or, because setting of standards was placed right after introducing the new task and task strategies, they may have been receptive to the information provided but did not process it in a manner that allowed standards to influence their further action. We regret not having asked students after the experiment explicitly about the standards they had employed. This could have clarified the reason underlying the lack of effects. Since prior research (Kitsantas \& Zimmerman, 2006) has shown that externally set self-evaluative standards do influence learning within a cycle of self-regulation, it can be assumed that the operationalization of standards in the present study was suboptimal.

Surprisingly in this context, we found the hypothesized additive effect of mastery learning standards and individual feedback on only one of the dependent measures. The effect occurred for self-evaluative accuracy, and solely in overconfident students. It is difficult to explain this result considering that we did not find any further effects of self-evaluative standards in neither the complete sample nor the at-risk group. Further studies are therefore desirable to establish the connection between externally set standards and self-regulation in early adolescents.

The second objective of this study was to explore the effects of individual and social comparison feedback on students' mathematical performance and self-regulatory processes. Basically, it was hypothesized that feedback presented in the form of a graph over multiple tasks can enhance a learner's metacognitive awareness of his or her performance and thereby improve achievement calibration. Exploratively, we further addressed the question as to whether social comparison feedback is as supportive as individual feedback. In support of our hypothesis, students who received either social comparison feedback or individual feedback were more accurate and less biased in their self-evaluative judgements than students in the control group. Additionally, in overconfident students social comparison feedback led to higher self-efficacy accuracy and less bias. It is possible that those who received social comparison feedback had benefited from the information about others' performance. Presumably, this information helped them to develop a more realistic concept of their accomplishment on the task, possibly associated with reflecting its requirements. This, in turn, may not only have led to increased postdictive accuracy, but also made their predictions more accurate. It can be 
assumed that this effect was confined to the at-risk group of overconfident students because those who usually perform well and possess the metacognitive skills to conduct an accurate task analysis do not need to rely on the information about others' performance. Thus, they do not benefit from this additional component of feedback.

To summarize, while in the complete sample feedback affected only postdictive accuracy, in overconfident students it led to both higher predictive and postdictive accuracy. Another general finding that fits into the picture, was that those who received either type of feedback were more able to give a reasonable verbal explanation for their rating of the perceived quality of their performance than the control group. They appeared to be more aware of the process of self-evaluation. Our results suggest that the degree to which learners monitor their performance and hence become better calibrated can be enhanced by providing outcome feedback over multiple tasks and present it as visual graphs. Contrary to our results, a study by Schraw et al. (1993) revealed no effects of outcome feedback on calibration. Hacker et al. (2000) report that only high performing students benefited from feedback that was provided over multiple tests. However, the present study has been able to demonstrate that gains in calibration can be achieved by providing feedback. Moreover, it can be assumed that graphing is of particular support of fifth-grade students' self-observation and self-evaluation processes. Presented as a type of feedback that goes beyond mere outcome feedback, this procedure appears to be a powerful support of students' self-monitoring. This finding extends prior research on the impact of graphing (Kitsantas \& Zimmerman, 2006) and self-recording (Kitsantas \& Zimmerman, 1998; Zimmerman \& Kitsantas, 1997) to the domain of mathematics and the population of early adolescents who lack the metacognitive skills to make accurate judgements about their capability.

Gains in calibration accuracy went hand in hand with increased problem solving performance. However, this effect was only found in overconfident students and was confined to social comparison feedback. One explanation is that according to social cognitive theory and research, learners' performance becomes progressively more self-regulated (Zimmerman \& Kitsantas, 1997, 1999). The proposed levels of self-regulation represent increasing metacognitive, motivational, and behavioral regulation of a domain-specific skill. Accordingly, average and high performing students are supposed to be at a higher level of regulation and hence are able to apply strategies and complete tasks rather automatically. As a result, they do not benefit from additional support of metacognitive awareness. In contrast, the at-risk students in the present study can be assumed to be at a lower level of regulation. These students, who 
show less automaticity in problem solving, may have benefited from feedback as it enhanced their awareness of the requirements of the task and increased their self-monitoring.

The presence of social comparison information appeared to have rather supportive than detrimental effects on the development of skill. A possible explanation is that this type of feedback provided students with additional information about how well others perform and what is a realistic outcome, which seems to be of particular relevance for overconfident students. At the same time, graphing the results retained a focus on the personal development of skill. Within the framework of a program to empower students to self-regulate their learning, Cleary and Zimmerman (2004) recommend this method to support especially at-risk students. In terms of the impact of feedback on self-satisfaction, results again suggest an explanation derived from the theory of self-regulation (Zimmerman, 2000). Assuming that people seek information in order to evaluate their performance (Bandura \& Jourden, 1991; FranceKaatrude \& Smith, 1985; Wood, 1989) and that inducing standards in our study was not successful, students probably formed self-evaluative standards spontaneously. Herein, their standards were based on available information (e.g., feedback). According to the model of selfregulation (Zimmerman, 2000), learners' level of self-satisfaction results from comparing the observed outcome against a standard. Students who received individual feedback turned out to be most dissatisfied. This rather contradictory result may be due to the fact that externally set standards did not exert any influence on students' self-regulation. Students in the individual feedback group may therefore have used the information available to them and adapted an absolute standard, that is, the maximum score (5 points each trial). Kitsantas and Zimmerman (2006) report that setting absolute self-evaluative standards has detrimental effects on learners' motivation and learning outcomes, whereas graduated and realistic standards lead to increased satisfaction and performance. Students who received social comparison feedback may have formed a self-evaluative standard based on the social comparison information. It may be that these students benefited from the fact that the group's mean was lower than the maximum (five points). Therefore, the standard evolved was not too high and rather graduated than absolute in nature. Students in the control group who did not receive any feedback did have neither criterial nor social comparative information about a possible standard. These students' level of self-satisfaction was comparable to the level of students in the social comparison feedback group. Their high level of satisfaction may be due not having received any feedback, and as a result they did not get any information about failure either. Hence, these students were presumably less critical about their performance. 
Our data indicate that individual feedback leaves students most dissatisfied with their performance. However, in the context of the positive effects of feedback on calibration accuracy, we suggest that a certain level of dissatisfaction is not necessarily problematic. These students may be more willing to improve their performance on subsequent trials. However, in order to support not only cognitive, but also motivational aspects of learning, individual feedback should to be presented in a way that helps students to value their development of skill (Cleary \& Zimmerman, 2004) and to focus on graduated standards (Kitsantas \& Zimmerman, 2006). In our study, there might have been the risk that students in the individual feedback group became hypercritical about themselves, reflected by their low level of satisfaction.

Taken together, the results clearly indicate that feedback positively influences both self-regulation and performance. At a first glance, feedback in the presence of social comparison seems to be even more supportive than individual feedback. However, we suggest taking a closer look at what the results are telling us. Clearly, there are certain aspects in social comparison feedback that might be beneficial for self-regulation. It provides students with information that can be used in the formation of self-set standards for evaluating own performances (France-Kaatrude \& Smith, 1985). Moreover, in some cases social comparative information may prevent students from employing absolute unattainable self-evaluative standards by indicating that others do not necessarily perform at a maximum level. While these aspects may be supportive of self-regulation and motivation, social comparison in the classroom can have detrimental effects as well (e.g., Ames \& Ames, 1978; France-Kaatrude \& Smith, 1985). This especially occurs in the case of permanent failure. According to Bandura and Jourden (1991, p. 950), 'it remains a challenge as how to minimize the demoralizing effects of unfavorable social comparison.' Our results suggest that the presence of social comparison is not necessarily detrimental for self-regulation. However, at the same time information about learning outcomes has to be presented in a way that puts greater emphasis on indicants of personal improvement. One means to achieve this is graphing.

In conclusion, the present study provides support for a cyclical model of selfregulation (Zimmerman, 2000). In this cycle, calibration is an inherent component. Enhancing self-monitoring during performance by providing graphed feedback not only increased calibration accuracy, but also improved at-risk students' problem solving performance. Furthermore, according to the model, students use a standard to evaluate their performance. In absence of externally set standards, self-set standards are employed. Clearly, results highlight the relationship between phase-related self-regulatory processes. 
These findings have educational implications. With calibration accuracy being highly relevant for self-regulation, teachers should attempt to enhance metacognitive skills that allow students to monitor and accurately evaluate their performance. Presenting feedback in the form of a graph is an appropriate method to provide information about both learning outcomes and personal development of skill. Positive effects can be achieved even in the presence of social comparison, which makes the application of graphs as a means of feedback a useful element of everyday activities in the classroom. Moreover, graphing can be particularly beneficial for students of lower levels of academic achievement who overestimate their capabilities because it facilitates the development of a sense of control over learning outcomes. Educators should further keep in mind that the self-evaluative standards students employ has important implications for their emotional and motivational reactions after performance. Hence, standards should be clearly communicated to students, realistic to achieve as well as sensitive to small improvements in skill (Kitsantas \& Zimmerman, 2006).

However, our data need to be interpreted with caution. Results are subject to contextual constraints and therefore not directly transferable to actual classroom settings. While students in our study were tested individually and social comparison feedback was rather artificial, in actual classrooms there are much more complex influences that affect learners' cognitive and motivational processes. Nevertheless, the present research is an initial effort to study the effects of influences that are present in classrooms on self-regulatory processes in students. The next step could be a study that specifically targets the effects of graphed feedback in actual class. This would contribute to our knowledge of how to promote self-regulated learning in regular classrooms.

We separately conducted analyses for the selected group of overconfident students. As a result, number of students in each experimental group slightly varied. Although this is not a fortunate condition to perform analyses of variance, we consider this method an appropriate way to broaden our knowledge about learning processes in overconfident students.

Another limitation of this study is the small number of students in our sample who are accurate or underconfident in their self-judgements. Hence, it was not possible to include $d i$ rection of bias as a factor in the analyses. Further studies are recommended to investigate differences in self-regulatory processes between overconfident, underconfident, and well calibrated students. Research questions that could be addressed include whether students at different levels of academic achievement, calibration, and self-regulatory skills benefit differently from various types of feedback, i.e. outcome feedback, process feedback, and feedback in the presence or absence of social comparison. Future research that takes into account both 
characteristics of classrooms and self-regulatory processes in individual learners will contribute profoundly to developing interventions and designing lessons to teach students to become responsible and self-directed learners. 


\section{$7 \quad$ Resümee und Ausblick}

In diesem abschließenden Kapitel werden die Forschungsfragen der vorliegenden Arbeit aus Kapitel 3.3 wieder aufgegriffen und die in den empirischen Teilen aufgezeigten Ergebnisse synoptisch dargestellt und diskutiert (7.1). Ausgehend von den zentralen Befunden wird in Kapitel 7.2 auf Implikationen für die pädagogisch-psychologische Praxis eingegangen. Die Arbeit schließt mit Perspektiven für zukünftige Selbstregulationsforschung an der Schnittstelle zwischen Pädagogischer Psychologie, Entwicklungspsychologie und den Fachdidaktiken (7.3).

\subsection{Zusammenfassung und Diskussion}

Die zentralen Ergebnisse zu Frage 1 beziehen sich auf den ersten empirischen Beitrag in Kapitel 4.1 (Labuhn, Bögeholz \& Hasselhorn, 2008). Die relevanten Befunde zu den Fragen 2a, $2 \mathrm{~b}$ und 3 stellt der zweite empirische Beitrag in Kapitel 4.2 (Labuhn, Bögeholz \& Hasselhorn, in Druck) bereit. Die in Kapitel 6 berichtete Studie (Labuhn, Zimmerman \& Hasselhorn, submitted) liefert Antworten auf die Forschungsfrage 4.

\section{(1) Lässt sich selbstreguliertes Lernen unterrichtsintegriert ohne negative Auswirkungen} auf den kurzfristigen fachlichen Wissenserwerb wirksam fördern?

Die relevanten Befunde zu dieser Frage lauten zusammengefasst:

a) Der signifikante Anstieg des Gesamtwertes SRL bei trainierten Schüler(inne)n belegt die prinzipielle Möglichkeit eines Zugewinns an Selbstregulationsvermögen durch die Intervention.

b) Auf Subskalenebene weisen der tendenzielle Anstieg in den Selbstwirksamkeitserwartungen sowie der signifikante Zuwachs berichteter Lernstrategienutzung darauf hin, dass in diesen Bereichen ein Aufbau von Selbstregulation angestoßen werden kann.

c) Im direkten Anschluss an die Intervention zeigte sich kein Unterschied zwischen Trainings- und Kontrollgruppe im ernährungsbezogenen Wissen. Dieses Resultat bestätigt, dass das fachbezogene Lernergebnis in der Phase der unterrichtsintegrierten Selbstregulationsförderung nicht schlechter ausfällt. 
(2a) Führt die zeitlich begrenzte unterrichtsintegrierte Selbstregulationsförderung längerfristig zu einem Aufbau der Selbstregulationsfähigkeit?

Die Analyse der Follow-up-Daten, erhoben ein Schulhalbjahr nach der Intervention, lieferte für diese Frage folgende zentrale Befunde:

a) Der durch das Training erzielte Anstieg der Selbstregulation ließ zwar in seiner absoluten Höhe über einen Zeitraum von sechs Monaten nach, trotz der Abnahme blieb der signifikante Vorteil der Trainings- gegenüber der Kontrollgruppe im Gesamtwert SRL jedoch bestehen.

b) Der im direkten Anschluss an die Förderung gefundene signifikante Vorteil der Trainingsgruppe für die Subskala Lernstrategien zeigte sich im Follow-up nicht mehr.

c) Für die Bereiche Selbstwirksamkeitserwartungen und Motivation waren sechs Monate nach der Intervention signifikante Vorteile der trainierten Schüler(innen) festzustellen.

(2b) Führt die zeitlich begrenzte unterrichtsintegrierte Selbstregulationsförderung längerfristig zu positiven Effekten auf den Lernerfolg?

Die im Hinblick auf die langfristige Wirkung der Intervention auf den Lernerfolg relevanten Ergebnisse lauten:

a) Bei der wiederholten Leistungsmessung sechs Monate nach der Intervention schnitt die Trainingsgruppe signifikant besser ab.

b) Der Mittelwert des fachbezogenen Tests lag sogar über dem im direkten Anschluss an die Unterrichtseinheit festgestellten Wert. Die Behaltensleistung der Trainingsgruppe überstieg damit die Wiedergabe des Gelernten kurz nach der Unterrichtseinheit.

(3) Ist die unterrichtsintegrierte Selbstregulationsförderung für Schüler(innen) mit unterschiedlichen Lernvoraussetzungen gleich wirksam?

Die differentiellen Analysen ergaben folgende zentrale Resultate:

a) Hinsichtlich der Wirksamkeit der Intervention auf die Selbstregulationsfähigkeit zeigte sich im Prä-Post-Vergleich kein signifikanter Unterschied zwischen Schüler(inne)n mit Migrationshintergrund und Schüler(inne)n ohne Migrationshintergrund.

b) In der Follow-up-Testung lag ein siginifikanter differentieller Effekt auf der Leistungsebene vor: Der deutliche Vorteil der trainierten Schüler(innen) im fachbezogenen Test war nicht für Kinder mit Migrationshintergrund nachzuweisen. Dieser Interakti- 
onseffekt war jedoch auf ein unerwartetes Absinken der Leistung der Schüler(innen) ohne Migrationshintergrund in der Kontrollgruppe zurückzuführen, der zu dem signifikanten Unterschied zur Trainingsgruppe führte. Der Befund kann also nicht als eine durch das Training forcierte Benachteiligung der Schüler(innen) mit Migrationshintergrund interpretiert werden.

c) Es zeigte sich kein „klassischer“ Matthäuseffekt: Schüler(innen), die vor der Intervention zu den „schwachen Selbstregulierern“ zählten, profitierten trotz der ungünstigeren Ausgangslage in vergleichbarem Maße wie die „starken Selbstregulierer“ von der Förderung.

d) Für die Schüler(innen), die vor der Intervention zu den „schwachen Selbstregulierern“ zählten, waren auch langfristig und bezogen auf die Entwicklung der fachbezogenen Leistung keine signifikanten Nachteile festzustellen, die auf ihr anfangs unterdurchschnittliches Selbstregulationsniveau zurückgingen.

\section{Zusammenfassende Diskussion zur unterrichtsintegrierten Selbstregulationsförderung}

Im Rahmen von Studie 1 erfolgte die Evaluation einer in den regulären Unterricht integrierten Förderung selbstregulierten Lernens. Diese Evaluation erfolgte anhand einer Reihe von Kriterien, die sich auf die Wirksamkeit der Trainingsmaßnahme und ihre Relevanz für den Schulkontext beziehen: Kurzfristige spezifische Wirksamkeit, Sicherstellung des fachbezogenen Wissenserwerbs, langfristige Wirksamkeit und Chancengleichheit. Zunächst ging es um die nahe liegende Frage, ob die Intervention spezifisch wirksam ist und zu der intendierten Verbesserung der Selbstregulation führt. Die Resultate des Prätest-Posttest-Vergleichs deuten insgesamt auf eine positive Wirkung der Unterrichtsintervention hin. Der signifikante Anstieg des Gesamtwertes für selbstreguliertes Lernen, der tendenzielle Zugewinn in den Selbstwirksamkeitserwartungen sowie der signifikante Anstieg der berichteten Lernstrategienutzung sprechen dafür, das erste Evaluationskriterium, die spezifische Wirksamkeit, als erfüllt zu betrachten.

Das zweite Kriterium für den Erfolg der Maßnahme bestand darin sicherzustellen, dass die Einbindung von Komponenten der Selbstregulationsförderung in den Unterricht nicht zu geringeren fachbezogenen Lernleistungen führt. Bei unterrichtsintegrierten Programmen kann es zu negativen Auswirkungen auf den Wissenserwerb kommen, da weniger Zeit für fachliches Lernen zur Verfügung steht und der Neuheitswert der Selbstregulationselemente zu Lasten der Konzentration auf fachbezogene Inhalte gehen kann. In der Phase des Erwerbs neuer Strategien kann es darüber hinaus kurzfristig zu einem Absinken der Leistungen kom- 
men (Hasselhorn \& Gold, 2006, S. 97ff.). Die Resultate des fachbezogenen Tests im direkten Anschluss an die Intervention zeigten jedoch, dass sich die Leistungen von Schüler(inne)n aus Trainings- und Kontrollgruppe nicht signifikant voneinander unterschieden. Damit ist das zweite Kriterium für den Erfolg des Programms erfüllt. Die zusätzliche Einbindung der Selbstregulationsförderung hat offenbar weder zuviel Unterrichtszeit beansprucht noch vom Erwerb relevanten Fachwissens abgelenkt.

Das dritte Erfolgskriterium stellte der Nachweis der langfristigen Wirksamkeit dar. Der Nutzen unterrichtsintegrierter Ansätze ist besonders hoch einzuschätzen, wenn nicht lediglich kurzfristige Effekte erzielt werden, sondern Selbstregulationsfähigkeit und Lernerfolg dauerhaft gesteigert werden können. Wie die Follow-up-Analyse ergab, ließ die durch das Training erzielte Wirkung in ihrer Höhe über einen Zeitraum von sechs Monaten zwar wieder nach, der signifikante Vorteil der Trainingsgruppe blieb jedoch bestehen. Der im direkten Anschluss an die Förderung gefundene Vorteil der Trainingsgruppe für die Subskala Lernstrategien zeigte sich im Follow-up nicht mehr. Die erzielte Wirkung war hier offensichtlich lediglich kurzfristiger Natur, was auf mangelnde oder gänzlich fehlende Transfer- und Trainingsmöglichkeiten für strategisches Lernen im regulären Schulalltag zurückzuführen sein könnte (vgl. Kapitel 4.4 für eine ausführliche Diskussion der Grenzen des Ansatzes). Für die Bereiche Selbstwirksamkeitserwartungen und Motivation waren erst sechs Monate nach der Intervention signifikante Vorteile der trainierten Schüler(innen) festzustellen. Durch die Intervention wurde im motivationalen Bereich offenbar eine Entwicklung angestoßen, die sich über einen längeren Zeitraum und über die Trainingsphase hinaus fortsetzte. Im Rahmen der Überprüfung der langfristigen Interventionswirkung auf die fachbezogenen Leistungen schnitt die Trainingsgruppe deutlich besser ab. Darüber hinaus lag der Mittelwert des fachbezogenen Tests über dem im direkten Anschluss an die Unterrichtseinheit gemessenen Wert. Die Behaltensleistung der Trainingsgruppe ging damit noch über die Wiedergabe des Gelernten im direkten Anschluss an die Unterrichtseinheit hinaus (ein ähnliches Resultat berichten Leopold, den Elzen-Rump und Leutner, 2007). Möglicherweise geht dieser Befund auf ein bereits im Unterricht erlangtes tieferes Verständnis der vermittelten Konzepte zurück, welches auf längere Sicht zu einer verbesserten Leistung führte. In der Zusammenschau der Resultate zur langfristigen Wirkung der unterrichtsintegrierten Förderung kann das dritte Erfolgskriterium ebenfalls als erfüllt betrachtet werden.

Das vierte Kriterium in der kritischen Überprüfung der Fördermaßnahme stellte die Gewährleistung der Chancengleichheit dar. Förderung, die in der Schule ansetzt, sollte so konzipiert sein, dass alle Teilnehmenden potenziell profitieren können und kein Matthäusef- 
fekt verursacht wird. Die differentiellen Analysen belegen insgesamt die Wirksamkeit der Unterrichtsintervention auch für Schüler(innen) mit Migrationshintergrund sowie für Schüler(innen) mit schwachen Ausgangsvoraussetzungen für selbstreguliertes Lernen. Durch die Trainingsmaßnahme wurde keine dieser Subgruppen spezifisch benachteiligt. Mit der Sicherstellung der Chancengleichheit ist auch das vierte Evaluationskriterium erfüllt.

Trotz der in Kapitel 4.4 ausführlicher dargestellten Grenzen der vorgestellten unterrichtsintegrierten Selbstregulationsförderung sind diese Ergebnisse positiv zu bewerten. Die interdisziplinäre Kooperation von Lehrkräften, Fachdidaktiker(inne)n und Psycholog(inn)en hat sich in der vorliegenden Untersuchung als Herausforderung und gleichzeitig zielführend für die Förderung lernrelevanter Fähigkeiten und Fertigkeiten im Unterrichtskontext herausgestellt. Die Entwicklung von curricular validen Unterrichtseinheiten mit integrierten Elementen zur Förderung selbstregulierten Lernens stellt eine große Chance für das Erreichen des übergeordneten Ziels dar, Schüler(innen) in ihrer Entwicklung zu selbstständig und selbstverantwortlich Lernenden zu unterstützen. 
(4) Welche Merkmale von Instruktion und von Einflüssen des sozialen Kontextes im Klassenraum unterstützen den Aufbau von Selbstregulation?

Die relevanten Befunde zu dieser Frage lauten zusammengefasst:

a) Die experimentelle Variation der von außen gesetzten Bewertungsstandards zeigte keine signifikanten Effekte auf Selbstregulation oder Leistung. Das Ergebnismuster ist aber dennoch vereinbar mit der Annahme, dass Standards im Selbstregulationsprozess von Bedeutung sind:

- Aufgrund fehlender externer Standards könnten die Lernenden einen internen Standard zur Bewertung der Qualität eigener Ergebnisse auf der Basis verfügbarer Informationen über die Leistungen anderer Schüler(innen) gebildet haben.

- Lag keine Information über die Leistung anderer Schüler(innen) vor, stand als Vergleichsstandard lediglich das erreichbare Maximum zur Verfügung. Dies führte zu signifikant unzufriedeneren Reaktionen auf die eigene Leistung.

b) Graphing-Feedback, das über mehrere Übungsdurchgänge hinweg jeweils im direkten Anschluss an die Aufgabenbearbeitung eines Durchgangs gegeben wurde, bewirkte im Vergleich zur Kontrollgruppe, die kein Feedback erhielt, signifikant genauere Einschätzungen der eigenen Leistungen (self-evaluation accuracy), also bessere metakognitive Fähigkeiten

c) Bei Schüler(inne)n, die zur Überschätzung der eigenen Leistung tendieren, bewirkte Graphing-Feedback zusätzlich

- signifikant genauere Vorhersagen über die eigenen Leistungen (self-efficacy accuracy) und

- signifikant bessere Leistungen in der Posttest-Messung mathematischer Fähigkeiten

d) Soziale Vergleichsinformationen in Form der Rückmeldung über die durchschnittliche Leistung der anderen Schüler(innen) wirkten sich nicht negativ auf Fähigkeitserwerb und Selbstregulation aus. Die Feedback-Bedingung mit sozialem Vergleich gestaltete sich allerdings durch das Graphing derart, dass die Rückmeldungen auch die individuelle, längsschnittliche Leistungsentwicklung neben dem interindividuellen, querschnittlichen Vergleich beinhalteten.

\section{Zusammenfassende Diskussion zur Bedeutung von Feedback und Bewertungsstandards}

Im Rahmen von Studie 2 wurde der Einfluss von Feedback und Bewertungsstandards auf die Selbstregulation und den Erwerb einer neuen mathematischen Fähigkeit bei Fünft- 
klässler(inne)n untersucht. Das Ziel war die Identifikation unterrichtsrelevanter Merkmale von Instruktion und von Einflüssen des sozialen Kontextes, welche die Leistungsentwicklung und den Aufbau von Selbstregulation begünstigen. Die angenommene Wirkung explizit von außen gesetzter Standards auf die Bewertung der eigenen Leistung zeigte sich in der Untersuchung nicht. Dennoch deutet das Ergebnismuster insgesamt auf die modelltheoretisch angenommene Relevanz von Bewertungsstandards im Selbstregulationsprozess hin: Da sich die experimentelle Variation von Bewertungsstandards als nicht effektiv herausgestellt hatte, war kein explizit gesetzter Vergleichsstandard gegeben und damit nicht eindeutig definiert, was eine „gute“ Leistung ausmacht. Gemäß der Theorie bilden Lernende unter solchen Bedingungen einen internen Standard auf der Basis verfügbarer Informationen aus, um die Qualität des eigenen Ergebnisses beurteilen zu können. Innerhalb der Studie standen einigen Schüler(inne)n Informationen über die durchschnittliche Leistung anderer zur Verfügung (s.u.). Sie konnten diesen Durchschnittswert als Standard zur Bewertung eigener Resultate heranziehen. Lag hingegen keine Information über die Leistung anderer Schüler(innen) vor, war die maximal erreichbare Punktzahl der einzige Anhaltspunkt für die Einschätzung der Qualität der Leistung. Diese aus der Selbstregulationstheorie (Zimmerman, 2000) hervorgehende Annahme fand empirische Unterstützung in der Betrachtung der emotional-motivationalen Reaktion, gemessen durch die Zufriedenheit mit dem eigenen Lernergebnis: Stand zur Bewertung lediglich der Vergleich mit dem erreichbaren Maximum und damit ein absoluter und hoher Standard zur Verfügung, schlug sich dies in deutlich unzufriedeneren Reaktionen auf die eigene Leistung nieder. Dieser Befund steht im Einklang mit dem Ergebnis einer Studie von Kitsantas und Zimmerman (2006), dass hohe und absolut formulierte Standards die Sensitivität für kleine Verbesserungen im Lernprozess einschränken und sich negativ auf Zufriedenheit und Leistung auswirken. Die Bewertungsstandards, die Schüler(innen) zur Einschätzung erzielter Resultate heranziehen, haben einen deutlichen Einfluss auf Selbstreflexionsprozesse, insbesondere emotionaler und motivationaler Art. Legt man das zyklische Selbstregulationsmodell (Zimmerman, 2000) zugrunde, wirken sich Standards vermittelt durch die Reaktionen auf die eigene Leistungsbewertung auf die nachfolgende Vorbereitungsphase und damit den weiteren Verlauf des Lernprozesses aus.

Als ein besonders relevantes Merkmal von Instruktion stellte sich externes (d.h. von außen bereitgestelltes) Feedback heraus. In der vorgestellten Studie erhielten die Schüler(inne)n über mehrere Übungsdurchgänge hinweg jeweils im direkten Anschluss an die Aufgabenbearbeitung die Rückmeldung über ihr erzieltes Ergebnis. Die jeweils erreichte Punktzahl wurde zusätzlich visuell in einem Koordinatensystem präsentiert und in Form eines 
Graphen abgetragen (Graphing, Kitsantas \& Zimmerman, 2006). Diese Art des Feedbacks bewirkte im Vergleich zur Kontrollgruppe deutlich bessere metakognitive Leistungen, festgestellt über die Genauigkeit der Einschätzung der eigenen Resultate der Bearbeitung von Mathematikaufgaben. Besonders effektiv erwies sich die Methode des Graphing-Feedback für eine Risikogruppe von Schüler(inne)n, die zur Überschätzung der eigenen Fähigkeiten bei gleichzeitig unterdurchschnittlichen Leistungen tendieren: In dieser Subgruppe führte Feedback, das außerdem die Information über die durchschnittliche Leistung der anderen Schüler(innen) enthielt, zusätzlich zu genaueren Vorhersagen für das eigene Lernergebnis und sogar zu besseren Leistungen in der Posttest-Messung mathematischer Fähigkeiten. Diese Schüler(innen) profitierten offenbar besonders von der über das Feedback bereitgestellten Unterstützung metakognitiver Komponenten des Self-monitoring und der Selbstreflexion. Die zusätzliche Information über die Leistung der anderen Schüler(innen) scheint eine stärkere Auseinandersetzung mit der Aufgabe und eine realistischere Betrachtung der Aufgabenanforderungen und der eigenen Fähigkeiten ermöglicht zu haben, was besonders in dieser Risikogruppe zu entscheidenden Verbesserungen führte. Auch in der Gesamtgruppe scheint die Vergleichsinformation eher einen Anhaltspunkt für die Einschätzung der Aufgabe und eigener Resultate bereitgestellt als demotivierend gewirkt zu haben. Sozialer Vergleich wirkt sich also nicht notwendigerweise negativ auf den Fähigkeitserwerb und die Selbstregulation aus. Wichtig ist jedoch hervorzuheben, dass die experimentelle Bedingung mit sozialen Vergleichsinformationen durch das Graphing-Feedback dadurch geprägt war, dass in den Rückmeldungen immer auch die individuelle, längsschnittliche Fähigkeits- und Fertigkeitsentwicklung neben dem interindividuellen, querschnittlichen Vergleich hervorgehoben wurde.

Zusammenfassend ist damit festzuhalten, dass Feedback und Bewertungsstandards zentrale, die Selbstregulation beeinflussende Elemente des Unterrichtskontextes darstellen, die entsprechend unterstützend gestaltet werden können. Extern gesetzte Standards sollten realistisch und nicht $\mathrm{zu}$ hoch oder absolut sein um nicht demotivierend zu wirken (vgl. Kitsantas \& Zimmerman, 2006). Graphing-Feedback stellt eine geeignete Methode zur Förderung metakognitiver Fähigkeiten (und in der Folge auch der Leistung) dar, die eine Ergebnisrückmeldung und die Betonung der individuellen Fähigkeitsentwicklung integriert. Der im Unterrichtskontext fast unvermeidbar vorhandene soziale Vergleich hat nicht per se eine negative Wirkung auf Selbstregulation und Leistung. Die Ergebnisse geben jedoch Anlass zu der Annahme, dass die Bedingungen gleichzeitig von der Betonung und Wertschätzung der individuellen Fähigkeits- und Fertigkeitsentwicklung geprägt sein sollten. GraphingFeedback kann hierzu einen Beitrag leisten. 


\section{Integrative Diskussion}

In der vorliegenden Arbeit wurden zwei in ihrer Konzeption sehr unterschiedliche Ansätze vorgestellt, deren gemeinsames Ziel das Aufzeigen von Möglichkeiten darstellte, selbstreguliertes Lernen im Unterrichtskontext zu fördern. Die Unterschiede der Ansätze bezogen sich in erster Linie auf a) die Art der Integration der Förderung in den Unterricht (das ,wie?“) und b) die Inhalte bzw. die spezifischen Wirkfaktoren der Intervention (das „was?“). Studie 1 verfolgte das übergeordnete Ziel durch die Integration der Selbstregulationsförderung in eine curricular valide Unterrichtseinheit im Fach Naturwissenschaften, also in schulische Alltagsbedingungen. Im Rahmen der Intervention wurden verschiedene Elemente der Förderung selbstregulierten Lernens (Zielsetzung, Motivation, Lernstrategien, Selbstreflexion, volitionale Strategien) direkt mit der Vermittlung fachbezogenen Wissens verknüpft. In Studie 2 ging es um die Identifikation von selbstregulationsförderlichen Merkmalen der Instruktion und des sozialen Kontextes, die unabhängig von sorgfältig geplanten Trainingsprogrammen in den regulären Unterricht integriert werden können. Die Untersuchung erfolgte im Kontext des Erwerbs einer neuen mathematischen Fähigkeit, für die untersuchten Mechanismen kann jedoch fächerübergreifende Gültigkeit angenommen werden. Beide Studien brachten Resultate hervor, die für den Erfolg der verschiedenen Versuche sprechen selbstreguliertes Lernen in direkter Verknüpfung mit der Vermittlung fachbezogenen Wissens zu fördern.

Die vorliegende Arbeit erweitert durch die Kombination der unterschiedlichen Forschungsansätze die Kenntnislage auf dem Gebiet der unterrichtsintegrierten Förderung selbstregulierten Lernens in mehrfacher Hinsicht. Es wurde gezeigt, dass die Förderung sowohl auf einer Makro- als auch auf einer Mikroebene ansetzen kann. Mit Makroebene ist die strukturelle Ebene der Planung und Konzeption von Unterrichtseinheiten mit einem Bezug zum Curriculum gemeint. Die Mikroebene bezieht sich auf spezifische Lernprozesse und die darauf bezogene selbstregulationsförderliche Gestaltung der Interaktion zwischen Lehrenden und Lernenden.

Evaluierte unterrichtsintegrierte Förderansätze (Makroebene) fanden bislang meist im Rahmen des Mathematik- oder Deutschunterrichts statt und bezogen sich auf stark strukturierte, homogene fachbezogene Inhalte wie mathematische Problemlösestrategien (Fuchs et al., 2003; Perels, Dignath \& Schmitz, in Druck), Textrevisionsstrategien (Glaser \& Brunstein, 2007a, 2007b) oder Strategien zur Förderung des Leseverständnis (Souvignier \& Mokhlesgerami, 2006; nach Schreblowski \& Hasselhorn, 2001). In der vorliegenden Arbeit wurde gezeigt, dass es möglich ist, die Selbstregulationsförderung mit dem heterogenen Stoff einer curricular validen Unterrichtseinheit im Fach Naturwissenschaften zu verknüpfen. Dieser An- 
satz bedeutet keinen grundlegenden Eingriff ins Curriculum, sondern lediglich die Integration von Selbstregulationskomponenten in die curricular vorgesehenen Themen. Die zusätzliche Einbindung selbstregulierten Lernens lässt sich derart gestalten, dass sie nicht zuviel Unterrichtszeit in Anspruch nimmt und sich damit nicht negativ auf den Erwerb fachbezogenen Wissens auswirkt. Langfristig kann die Selbstregulationsförderung sogar Lernvorteile bewirken.

Bislang lagen keine Befunde zur differentiellen Wirkung unterrichtsintegrierter Förderung vor. Ein weiteres wichtiges Teilergebnis der vorliegenden Arbeit ist daher der Befund, dass Schüler(innen) mit unterschiedlichen Lernausgangsvoraussetzungen von der Förderung auf Makroebene (Studie 1) sowie auf Mikroebene (Studie 2) profitieren und schwächere Lernende nicht spezifisch benachteiligt werden.

Auf Mikroebene zeigte sich, dass die in anderen Forschungsarbeiten (z.B. Kitsantas \& Zimmerman, 2006; Zimmerman \& Kitsantas, 1996) unterstützte Annahme der zyklischen Natur selbstregulierten Lernens auch auf den Erwerb einer neuen mathematischen Fähigkeit bei Fünftklässler(inne)n anzuwenden ist, und dass daraus selbstregulations- und leistungsförderliche Bedingungen für den Unterricht abgeleitet werden können. Selbstreguliertes Lernen kann also auch durch eine entsprechende Gestaltung instruktionalen Handelns unterstützt werden. Regelmäßiges Feedback und motivational günstige Bewertungsstandards stellen empirisch begründete Ansatzpunkte für die Förderung auf Lernprozessebene dar.

Effektive unterrichtsintegrierte Selbstregulationsförderung kann also auf unterschiedlichen Ebenen ansetzen und im Hinblick auf das Inhaltsspektrum variieren. Die vorgestellten Resultate stellen damit vielfältige Anknüpfungspunkte für die zukünftige Forschung und Förderung im Bereich selbstregulierten Lernens im Unterrichtskontext bereit.

Aus der Perspektive sozial-kognitiver Selbstregulationsforschung, die geprägt ist durch den Versuch, Lern- und Selbstregulationsprozesse im Detail zu verstehen um darauf aufbauend Möglichkeiten der Förderung zu entwickeln, soll nun abschließend noch ein kritischer Blick auf die beiden Studien geworfen werden. Da in Kapitel 4.4 sowie im Diskussionsteil von Kapitel 6 bereits näher auf die Grenzen jedes einzelnen Ansatzes eingegangen wurde, erfolgt hier lediglich eine komprimierte Zusammenschau. Tabelle 7.1 fasst gegenüberstellend zentrale Merkmale der Studien zusammen. Bereits bei einem ersten Blick auf die Übersicht fällt auf, dass Stärken und Schwächen der beiden Ansätze größtenteils komplementär zueinander sind. Hinsichtlich der Kriterien in den unteren vier Tabellenzeilen, Kontrolle von Störfaktoren, Validität der Messung, Korrelation zwischen SRL- und Leistungsmaßen sowie den Effektstärken, gehen die Ausprägungen dieser für empirische Studien wichtigen Qualitäts- 
merkmale zugunsten von Studie 2. Der große Vorteil dieser Studie ist aus Forschungsperspektive darüber hinaus in der eng an sozial-kognitive Prinzipien angelehnten Konzeption und der stringenten Ableitung der Feedbackintervention aus dem Selbstregulationsmodell zu sehen. Die Forschungsfragen knüpfen direkt an die Prozessannahme an, die Intervention erfolgt kontextspezifisch und fokussiert, die Messung verhaltensnah und valide (vgl. Tabelle 7.1, Kriteriumskorrelationen von bis zu $r=.60$ ). Diese inhaltlichen Aspekte in Verbindung mit den kontrollierten Rahmenbedingungen der Untersuchung spiegeln sich in großen Effektstärken wider. Der praktische Wert dieser Studie liegt im Aufzeigen von Möglichkeiten der Selbstregulationsförderung, die relativ einfach in reale Unterrichtssituationen $\mathrm{zu}$ integrieren sind. Feedback und die Berücksichtigung motivationsförderlicher Bewertungsstandards können als eher globale Prinzipien der Unterstützung selbstregulierten Lernens im regulären Unterricht betrachtet werden, die fächerübergreifende Gültigkeit besitzen und unabhängig von detailliert ausgearbeiteten Programmen an die spezifischen Kontexte zu adaptieren sind. Die im Rahmen von Studie 2 gewonnenen Daten erlauben aufgrund der Konzeption genaue und theorienahe Aussagen über den Selbstregulationsprozess, die aber gleichzeitig nicht ohne weiteres auf die reguläre Unterrichtssituation zu generalisieren sind. Unter Realbedingungen im Klassenraum unterliegen Lernprozesse vielen weiteren Einflussfaktoren, die ein Laborsetting nicht abzubilden in der Lage ist. In der ökologischen Validität liegt dagegen der entscheidende Vorteil von Studie 1. Die enge Kooperation mit Lehrkräften und die kontextspezifische Förderung von Selbstregulationselementen sind besondere Qualitätsmerkmale - der Schritt von der Grundlagenforschung in die Schulrealität ist als sinnvoll zu erachten und stellt auch für zukünftige Selbstregulationsforschung eine Herausforderung und Chance dar. Aufgrund der methodischen Grenzen von Studie 1, insbesondere der eingeschränkten Kontrolle von Störfaktoren sowie der Validitätsproblematik der Messung (vgl. Tabelle 7.1 sowie Kapitel 4.4), sind die Resultate trotz der hohen ökologischen Validität mit Vorsicht zu interpretieren.

Beide Studien haben damit spezifische Stärken im Hinblick auf den Erkenntnisgewinn im Bereich der sozial-kognitiv fundierten Selbstregulationsförderung. Studie 2 demonstriert, wie theoriegeleitete Konzeption und Messung realisiert werden können, Studie 1 ist ein Beispiel dafür, wie praktisch relevante Selbstregulationsforschung dort umgesetzt werden kann, wo der Forschungsbedarf der Zukunft liegt: unter Realbedingungen im Unterricht.

Für zukünftige Forschungsarbeiten wäre eine Integration der Stärken beider Ansätze wünschenswert, etwa in Form einer Validierung der in Studie 2 belegten zentralen Rolle von Feedback und unter Anwendung prozessorientierter, kontextspezifischer und handlungsnaher Messung von Selbstregulation und Leistung. 


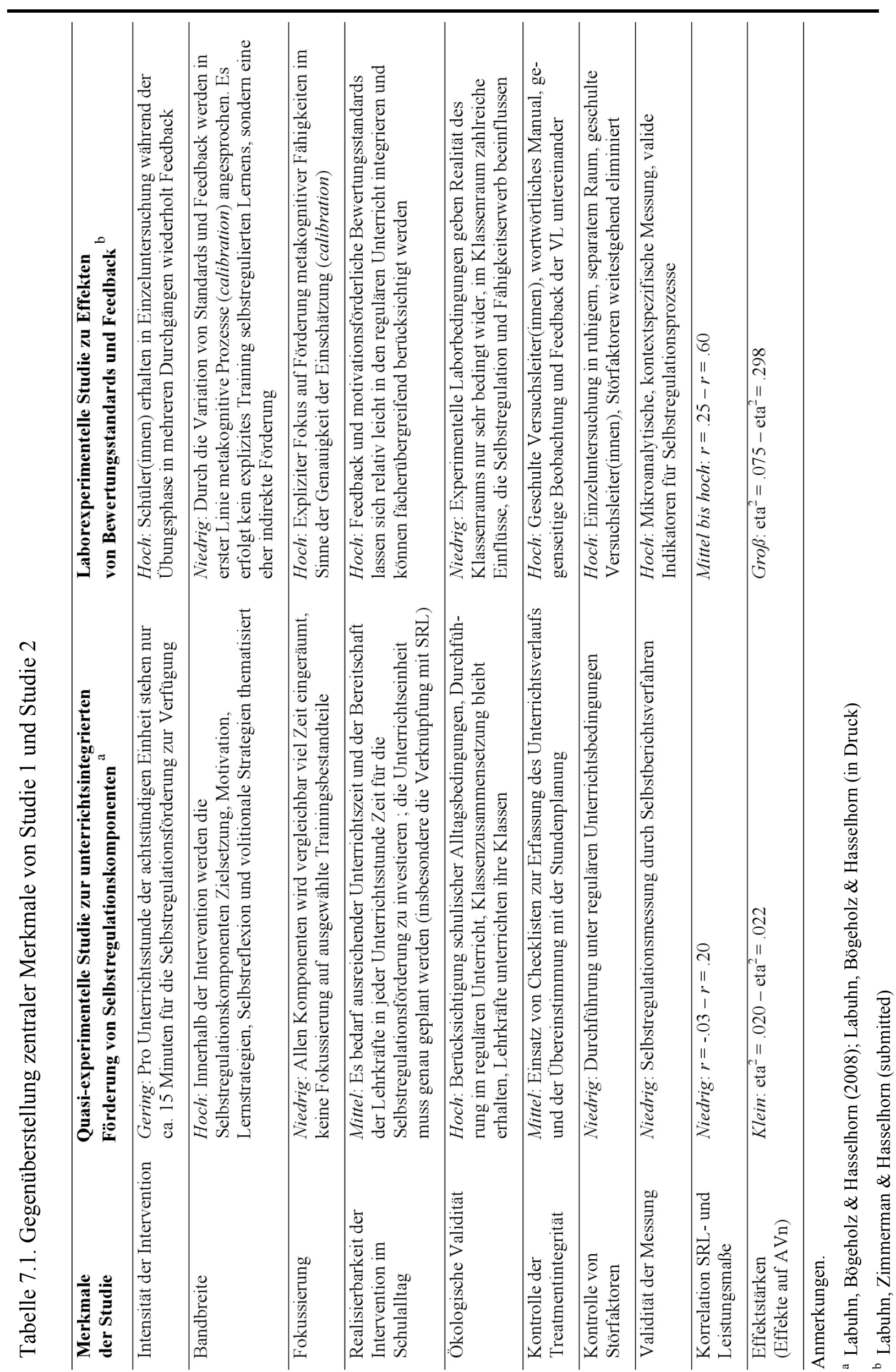




\subsection{Pädagogisch-psychologische Implikationen}

Die sozial-kognitive Theorie und die darin verankerte Konzeption selbstregulierten Lernens als ein zyklischer Prozess (Zimmerman, 2000) bilden geeignete Bezugspunkte für Überlegungen zum Aufbau und zur Förderung von Selbstregulationsfähigkeiten im Schulkontext. Die empirischen Beiträge der vorliegenden Dissertation wurden innerhalb dieses Bezugssystems entwickelt. Die Resultate unterstreichen dessen Relevanz für an der Fähigkeits-, Fertigkeits- und Selbstregulationsentwicklung interessierten Forschung und Praxis. In Kapitel 3 wurden Implikationen der sozial-kognitiven Theorie auf eher globaler Ebene herausgearbeitet (vgl. Tab. 3.1), die in die Konzeption der vorgestellten Studien eingeflossen sind. Nun geht es auf spezifischer Ebene um die pädagogisch-psychologischen Implikationen, die sich aus der vorliegenden Arbeit ergeben. Tabelle 7.2 stellt - ohne Anspruch auf Vollständigkeit eine Auswahl von Befunden und aus ihnen hervorgehende Implikationen dar.

Die empirischen Beiträge liefern Unterstützung für die Annahme, dass es sich bei selbstreguliertem Lernen um einen zyklischen Prozess handelt, der verschiedene Ansatzpunkte für die erfolgreiche Förderung einzelner Komponenten bietet. Im Rahmen von Studie 2 (vgl. Kapitel 6) konnte die Bedeutung von Feedback und Bewertungsstandards für den gesamten Selbstregulationszyklus demonstriert werden. Die Ergebnisse bestätigen die theoretische Annahme, dass nicht eine einmalige oder zeitlich eng begrenzte Förderung, sondern eine längerfristige Begleitung und Unterstützung dieses Prozesses sinnvoll ist. Das über einen Zeitraum von sechs Monaten beobachtete Absinken der Selbstregulationswerte und der Rückgang der berichteten Lernstrategienutzung nach der Unterrichtsintervention in Studie 1 (vgl. Kapitel 4.3) deuten auf die Notwendigkeit einer langfristigen Förderung mit wiederholten Anwendungsmöglichkeiten der Selbstregulationsfähigkeiten hin. In diesem Zusammenhang wäre es sinnvoll Lehrkräfte, die das selbstregulierte Lernen ihrer Schüler(innen) unterstützen möchten, mit dem zugrunde liegenden Modell und den Grundannahmen der Theorie vertraut zu machen. Auf diese Weise könnte das Verständnis des zyklischen Prozesses ihr Handeln leiten und die Förderung einzelner Selbstregulationskomponenten in einen größeren Zusammenhang gestellt werden (z.B. Zielsetzung und anschließende Reflexion; Standards und auf ihnen basierende Bewertung des Lernergebnisses; Bewertung und darauf folgende Adaption/ Modifikation der Herangehensweise). Wie aus der vorliegenden Arbeit hervorgeht, ist eine enge Anlehnung der Selbstregulationsförderung an den postulierten Prozess zielführend, sowohl auf der Ebene fachbezogener Leistungen als auch bezüglich der Entwicklung selbstregulierten Lernens. 
Die Frage ,Unterrichtsprogramme oder Unterrichtselemente?', d.h., ob der breit angelegte Ansatz in Form eines Programms aus mehreren Selbstregulationskomponenten (Studie 1) oder die spezifische Förderung eines fokussierten Bereichs (Studie 2) die Methode der Wahl darstellen, kann pauschal nicht beantwortet werden. Für die Praxis entscheidend ist die Orientierung am Prozessmodell der Selbstregulation und der daraus hervorgehenden Annahme, dass sich Effektivität und Effizienz des Lernens weiterentwickeln können, dazu aber Raum, Unterstützung und Zeit notwendig sind. Erst durch wiederholtes Üben und Anwenden neu erworbener Fähigkeiten und Fertigkeiten können diese einen Grad der Automatisierung erreichen, der eine selbstregulierte, erfolgreiche Anwendung erlaubt (Zimmerman, 2000). Dies spricht für eine intensivere Förderung der Selbstregulation über einen längeren Zeitraum hinweg - dabei spielt es eine untergeordnete Rolle, ob der Ansatz breit oder fokussiert gestaltet ist.

Unabhängig von der Möglichkeit, komplette Unterrichtsprogramme umzusetzen und ebenfalls weitgehend unabhängig von den Inhalten des Curriculums kann die Förderung selbstregulierten Lernens auch durch die Integration von Feedback in die Interaktion zwischen Lehrkräften und Schüler(inne)n realisiert werden. Regelmäßige und informative Rückmeldungen während des Lernprozesses unterstützen vermittelt durch die Anregung metakognitiver Überwachungsprozesse wie Self-monitoring die Entwicklung von Selbstregulation bei der Aufgabenausführung und verbessern in Folge dessen die Qualität der Ergebnisse. Dabei gilt es vor allem die individuelle Leistungsentwicklung und auch kleine persönliche Fortschritte hervorzuheben. Einen besonders viel versprechenden Ansatz, der regelmäßiges Feedback mit der Betonung des individuellen Lernprozesses verbindet, stellt die Methode des Graphing dar (Kitsantas \& Zimmerman, 2006). Cleary und Zimmerman (2004) zeigen, dass sich diese Form der Rückmeldung erfolgreich in der individuellen Arbeit mit einzelnen Lernenden verwirklichen lässt. Der Ansatz dürfte auch ins Unterrichtsgeschehen zu integrieren sein. Denkbar wäre weiterhin, Schüler(innen) eigenständig über einen längeren Zeitraum über erzielte Resultate Buch führen zu lassen.

Als ein weiteres wichtiges, den Selbstregulationsprozess beeinflussendes Element des Unterrichtskontextes haben sich Bewertungsstandards herausgestellt: Der Standard im Sinne einer „Messlatte“, an der Schüler(innen) erzielte Leistungen messen, fließt entscheidend in ihre Bewertung des eigenen Ergebnisses und damit in ihre emotional-motivationale Reaktion mit ein. Ist der Bewertungsstandard zu hoch, kann der Vergleich des erzielten Resultats mit diesem Sollwert zu Unzufriedenheit oder Resignation und damit zu negativen Auswirkungen auf den weiteren Verlauf des Lernprozesses führen. Kitsantas und Zimmerman (2006) schla- 
gen daher graduelle Standards vor, die Entwicklungsmöglichkeiten implizieren. Im Unterrichtskontext können solche graduellen Standards z.B. dadurch implementiert werden, dass für Aufgaben, die nicht vollständig gelöst wurden, Teilpunkte vergeben werden, wenn ein Lösungsansatz erkennbar ist. Denkbar wäre auch die Belohnung von Korrekturen zunächst falscher Lösungen, also die Unterstützung der eigenständigen Revision. Lehrkräfte sollten sich der Wirkung von Bewertungsstandards bewusst sein und Schüler(innen) darin unterstützen, individuelle Maßstäbe an die eigene Leistung anzulegen anstatt sich an einem absoluten Standard (z.B. der maximal erreichbaren Punkzahl oder der besten Note) zu orientieren. Die Bedeutung von Standards im Selbstregulationsprozess basiert auf dem Bestreben von Personen, die Qualität eigener Leistungen einzuschätzen. Eine Möglichkeit zu solchen Einschätzungen zu gelangen ist der Vergleich mit anderen Personen (vgl. die Theorie sozialen Vergleichs, Festinger, 1954). Im Klassenraum ist ein gewisses Maß an sozialem Vergleich beinahe unvermeidbar. Gemäß den Grundgedanken der sozial-kognitiven Theorie und verankert im Prinzip des reziproken Determinismus hat der soziale Kontext einen starken Einfluss auf individuelle Lernprozesse. Die Ergebnisse von Studie 2 zeigen, dass die Kenntnis der von Mitschüler(inne)n erzielten Resultate nicht notwendigerweise negative Konsequenzen für den Selbstregulationsprozess hat: Der Schwierigkeitsgrad von Aufgaben und damit das notwendige $\mathrm{Ma}$ an zu investierender Anstrengung sind besser abzuschätzen. Darüber hinaus können Lernende eine Vorstellung davon entwickeln, was realistischerweise erreichbar ist und damit ein adäquates Ziel darstellt (France-Kaatrude \& Smith, 1985). Das Wissen über Leistungen anderer (sofern sie nicht überdurchschnittlich gut sind) kann damit der Orientierung an zu hohen Bewertungsstandards entgegenwirken. Vor diesem Hintergrund gilt es im Klassenraum die potenziell günstigen Effekte des vorhandenen sozialen Vergleichs nutzbar zu machen z.B. durch kooperatives Lernen, in dessen Rahmen die Betonung kompetitiver Anteile reduziert wird. Grundsätzlich ist es aus der Perspektive der Selbstregulationsförderung von besonderer Bedeutung in der Kommunikation mit den Lernenden stets die individuelle Leistungsentwicklung stärker $\mathrm{zu}$ gewichten als den interindividuellen Vergleich zwischen Schüler(inne)n. Die Methode des Graphing stellt auch hinsichtlich dieses Ziels eine geeignete Möglichkeit der praktischen Umsetzung dar. 
Tabelle 7.2. Zentrale Befunde und Implikationen

\begin{tabular}{|c|c|}
\hline Befund & Implikation \\
\hline $\begin{array}{l}\text { Unterstützung der Annahme des } \\
\text { zyklischen Modells }\end{array}$ & $\begin{array}{l}\text { - Konzeption von Interventionen an das Prozessmodell anlehnen und } \\
\text { Elemente der Förderung in den zyklischen Zusammenhang stellen } \\
\text { - } \quad \text { Lehrkräfte oder Trainer(innen) sollten mit den Grundannahmen der } \\
\text { Theorie vertraut sein }\end{array}$ \\
\hline $\begin{array}{l}\text { Erfolgreiche unterrichtsintegrierte } \\
\text { Förderung von Selbstregulations- } \\
\text { elementen }\end{array}$ & $\begin{array}{l}\text { - Weiterführung und Ausbau des Ansatzes auf wissenschaftlicher } \\
\text { sowie auf praktischer Ebene } \\
\text { · } \\
\text { Interdisziplinäre Zusammenarbeit stärken } \\
\text { - Förderung auch in andere Fächer integrieren und transferförderliche } \\
\text { Bedingungen schaffen }\end{array}$ \\
\hline $\begin{array}{l}\text { Langfristige Wirksamkeit der In- } \\
\text { tervention bei Absinken des } \\
\text { Niveaus }\end{array}$ & $\begin{array}{ll}\cdot & \text { Langfristige Förderung aufbauen } \\
\text { · } & \text { Anwendungsmöglichkeiten für Selbstregulationselemente im } \\
\text { regulären Unterricht unabhängig von ausgearbeiteten Programmen } \\
\text { schaffen } \\
\text { · Vernetzung der Fachlehrkräfte, fächerübergreifende Förderung und } \\
\text { Transferförderung }\end{array}$ \\
\hline $\begin{array}{l}\text { Unterstützung der metakognitiven } \\
\text { Prozesse durch Feedback }\end{array}$ & $\begin{array}{l}\text { - Regelmäßiges, handlungsnahes Feedback unabhängig von Noten- } \\
\text { vergabe in den Unterricht integrieren } \\
\text { - Feedback individuell gestalten und den Lernprozess hervorheben } \\
\text { - } \quad \text { Graphing-Feedback als viel versprechender Ansatz, der regelmäßi- } \\
\text { ges Feedback mit der Betonung des individuellen Lernprozesses } \\
\text { verbindet }\end{array}$ \\
\hline $\begin{array}{l}\text { Indirekte Wirkung von Bewer- } \\
\text { tungsstandards }\end{array}$ & $\begin{array}{l}\text { Externe Standards transparent kommunizieren (um selbst gesetzten, } \\
\text { internen und u.U. zu hohen Standards vorzubeugen) } \\
\text { Bewertungsstandards sollten realistisch, graduell und nicht zu hoch } \\
\text { oder absolut sein } \\
\text { Schüler(innen) darin unterstützen, adäquate Standards anzulegen und } \\
\text { auf darauf aufbauend zur Reflexion der eigenen Leistung anregen }\end{array}$ \\
\hline $\begin{array}{l}\text { (Mögliche) günstige Wirkung soz- } \\
\text { ialen Vergleichs }\end{array}$ & $\begin{array}{l}\text { Demotivierende Wirkung sozialen Vergleichs (z.B. durch Vergleich } \\
\text { mit zu hohen sozialen Standards) zu reduzieren versuchen } \\
\text { Individuelle Entwicklung stärker hervorheben und wertschätzen als } \\
\text { interindividuellen Vergleich }\end{array}$ \\
\hline $\begin{array}{l}\text { Vergleichbare Effektivität der } \\
\text { Intervention für Risikogruppen }\end{array}$ & $\begin{array}{l}\text { Selbstregulationsförderung im Unterrichtskontext sinnvoll und } \\
\text { zugleich eine Chance, alle Schüler(innen) zu erreichen } \\
\text { · Potenzial der Selbstregulationsförderung für Unterstützung von } \\
\text { Risikogruppen ausschöpfen, z.B. durch die Entwicklung zusätzli- } \\
\text { cher, spezieller Programme }\end{array}$ \\
\hline
\end{tabular}


Unterrichtsintegrierte Interventionen, welche die Förderung selbstregulierten Lernens in Verknüpfung mit entsprechenden bereichsspezifischen Inhalten realisieren, werfen die Frage nach dem Transfer der erworbenen Selbstregulationsfähigkeit auf. Es wäre wünschenswert, dass die im Kontext eines bestimmten Faches aufgebaute Selbstregulation auch auf andere Inhaltsgebiete transferiert wird. Da die Förderung zunächst kontextspezifisch und in direkter Verknüpfung mit fachspezifischen Inhalten erfolgen sollte, besteht eine besondere Herausforderung darin, die Vermittlung so zu gestalten, dass erworbene Selbstregulationskomponenten transferfähig werden. Nach Pressley et al. (1990) hängt der Transfer selbstregulierten Lernens auf andere Kontexte, Fachgebiete und Aufgaben neben dem Wissen über geeignete Strategien auch von der Fähigkeit ab, den Selbstregulationsprozess der jeweiligen Situation und Aufgabe entsprechend anzupassen. Möglichkeiten des Transfers sollten also anfänglich explizit aufgezeigt werden um die Schüler(innen) dafür zu sensibilisieren, dass die erworbenen Fähigkeiten (z.B. Zielsetzung, Self-monitoring, Reflexion) flexibel einsetzbar sind. Lehrkräfte können diesen Prozess durch Modellverhalten unterstützen, z.B. indem sie die Anwendung von Selbstregulationsstrategien auch an anderen Aufgaben oder Inhalten demonstrieren. Besonders wünschenswert wäre eine fächerübergreifende Transferförderung. Durch eine Vernetzung und Kooperation von Lehrkräften wäre es möglich in verschiedenen Fächern Anwendungsmöglichkeiten in den Unterricht zu integrieren und damit die flexible Nutzung der erworbenen Selbstregulationsfähigkeiten zu unterstützen (vgl. Schunk \& Pajares, 2004). Im Hinblick auf die Konzeption der Entwicklungsniveaus der Selbstregulation nach Zimmerman (2000) stellen die genannten Punkte (Verhaltensmodelle, multiple Anwendungsmöglichkeiten, Förderung der Flexibilität) sinnvolle Schritte zum Erreichen eines hohen Niveaus selbstregulierten Lernens dar.

Abschließend soll in Bezug auf die Implikationen, die aus den empirischen Beiträgen dieser Arbeit hervorgehen, noch die Zielgruppe von Selbstregulationsinterventionen angesprochen werden: Diese bilden zunächst einmal alle Lernenden. Die Befunde beider Studien deuten darauf hin, dass Ansätze zur Förderung selbstregulierten Lernens Schüler(innen) mit unterschiedlichen Leistungs- und Selbstregulationsvoraussetzungen vergleichbar gut ansprechen ${ }^{9}$. Die Tatsache, dass sich keine spezifische Benachteiligung von Subgruppen zeigt, spricht dafür, Fördermaßnahmen in Zukunft verstärkt in den regulären Unterricht zu integrieren. Die Ergebnisse von Studie 2 deuten zusätzlich auf den Gewinn der Selbstregulationsför-

\footnotetext{
9 Studie 1: Schüler(innen) mit Migrationshintergrund sowie „schwache Selbstregulierer“; Studie 2: Schüler(innen) mit - relativ zur Gruppe der Gymnasiast(inn)en - unterdurchschnittlicher Leistung, die gleichzeitig zur Überschätzung eigener Fähigkeiten tendieren
} 
derung besonders für schwächere Schüler(innen) hin. Interventionen zur Unterstützung der Selbstregulation, also metakognitiver, motivationaler und behavioraler Aspekte des Lernens, könnten besonders für die Arbeit mit Risikogruppen (d.h. Schüler(innen) mit verschiedenen lern- und entwicklungsbezogenen Defiziten) Erfolg versprechende Ansätze der Förderung darstellen.

\subsection{Ausblick}

Die vorliegende Arbeit leistet einen Beitrag zum Verständnis des zyklischen Prozesses selbstregulierten Lernens und zur Entwicklung aus diesem Verständnis hervorgehender Möglichkeiten der unterrichtsintegrierten Selbstregulationsförderung. Die gewonnenen Erkenntnisse stellen einen weiteren Schritt in die Richtung des übergeordneten Ziels dar, junge Menschen in ihrer Entwicklung zu selbstverantwortlich Lernenden zu unterstützen. Der Schule kommt dabei eine besonders wichtige Funktion zu, da sie einen Ort darstellt, an dem potenziell alle Kinder erreicht werden können - auch jene, die hinsichtlich der Unterstützung aus dem Elternhaus eher benachteiligt sind. Vor dem Hintergrund der empirischen Belege, dass selbstreguliertes Lernen an unterschiedlichen Ansatzpunkten innerhalb des regulären Unterrichts gefördert werden kann, sollte die zukünftige Forschung an der Schnittstelle zwischen den Fachdidaktiken, der Entwicklungspsychologie und der pädagogischen Psychologie fortgeführt und ausgebaut werden. Im Hinblick auf von Lehrkräften und Wissenschaftler(inne)n kollaborativ konzipierte Unterrichtsprogramme wäre es wünschenswert, Elemente der Selbstregulationsförderung zu identifizieren, die sich besonders gut für die Integration in den Fachunterricht eignen und zeitlich stabile Effekte erzielen (vgl. dazu Zeidner, Boekaerts \& Pintrich, 2000) So könnte die Förderung fokussierter und idealer Weise sogar fächerübergreifend gestaltet werden. Besonders im Hinblick auf die Vereinbarkeit von Fachunterricht und transferfähiger Lernförderung ist dies ein relevantes Ziel. Die Befunde zur positiven Wirkung von Feedback im Lernprozess deuten auf das Potenzial des Ansatzes hin, universelle Elemente der Selbstregulationsförderung zu identifizieren, die in direkter Verknüpfung mit entsprechenden fachbezogenen Inhalten umzusetzen sind. Sofern sie als Elemente in die Instruktion integriert werden können, kosten sie kaum zusätzliche Unterrichtszeit.

Gleichzeitig wäre es interessant zu ermitteln, ob es neben universellen Elementen auch fachspezifische Kompetenzen gibt, die sich besonders gut mit den Grundgedanken der Selbstregulation verbinden lassen und damit weitere Ansatzpunkte der fokussierten Förderung bereitstellen. Mit dem Ziel, selbstreguliertes Lernen erfolgreich in den Unterrichtskontext zu 
integrieren, könnte interdisziplinäres Arbeiten und Forschen schon in der Ausbildung angehender Lehrkräfte und Psycholog(inn)en angelegt werden. Denkbar wäre die gemeinsame Entwicklung einer Art „Werkzeugkasten“, der Lehrkräften aller Fachdisziplinen Elemente der Selbstregulationsförderung bereitstellt, die entsprechend der jeweiligen Zielgruppe und Themenschwerpunkte ausgewählt und kontextspezifisch angepasst werden können (z.B. Feedback). In diesem Zusammenhang könnte auch der Frage nachgegangen werden, wie eine Förderung gestaltet sein sollte, die insbesondere die bereits angesprochenen Risikogruppen in ihrer Entwicklung unterstützt. Forschungsergebnisse deuten auf das Potenzial der Unterstützung der Selbstregulation von Kindern mit Lernschwierigkeiten hin (Klassen, 2002, 2006, 2007).

Hand in Hand mit der Weiterentwicklung von Ansätzen der unterrichtsintegrierten Förderung selbstregulierten Lernens sollte die Auseinandersetzung mit dessen Messung gehen. Das fordern auch Zeidner et al. (2000) in einer Zusammenstellung von Herausforderungen für zukünftige Selbstregulationsforschung. Nur über valide und veränderungssensitive Messinstrumente werden praxisrelevante Interventionen der Evaluation und damit der Qualitätssicherung und Weiterentwicklung zugänglich. In Zukunft sollte der sozial-kognitiv fundierten, kontextspezifischen und prozessorientierten Messung von Selbstregulationskomponenten verstärkte Aufmerksamkeit gewidmet werden. Die Anwendung mikroanalytischer Methoden, die einen handlungsnahen Zugang zum Selbstregulationsprozess erlauben, dürfte entscheidend zum Erkenntnisgewinn beitragen und gleichzeitig weitere Ansatzmöglichkeiten für Interventionen aufzeigen. An diesem Punkt laufen die Interessen von Forschung und Praxis zusammen. Eine valide Messung, die genaue Einblicke in die Selbstregulationsfähigkeit Lernender erlaubt, erfüllt zum einen eine wichtige Grundvoraussetzung aussagekräftiger Selbstregulationsforschung, zum anderen stellt sie auch Lehrkräften relevante Informationen zur Verfügung, die über die Resultate regulärer Verfahren zur Leistungsmessung hinausgehen und es ihnen erlauben Schüler(innen) individuell und umfassend zu fördern (vgl. Cleary \& Zimmerman, 2006). Sowohl im Hinblick auf die Messung, als auch bezüglich der Konzeption von Interventionen wird deutlich, dass der Fortschritt im Bereich der unterrichtsintegrierten Selbstregulationsförderung zu einem entscheidenden Anteil von einer interdisziplinären $\mathrm{Zu}-$ sammenarbeit von Wissenschaftler(inne)n und Lehrkräften abhängen wird, die das gemeinsame Ziel verfolgen, den Selbstregulationsprozess in seiner Komplexität zu verstehen um ihn zugunsten der Lernenden zu optimieren. 


\section{Zusammenfassung}

Ausgehend von Forschungsergebnissen, die auf einen positiven Einfluss selbstregulierten Lernens auf schulische Leistungen hindeuten, war das Ziel der vorliegenden Arbeit, verschiedene Ansätze der Selbstregulationsförderung im regulären Unterricht aufzuzeigen. Die untersuchten Ansätze unterschieden sich hinsichtlich der Art der Integration der Förderung in den Unterricht und den Inhalten bzw. den spezifischen Wirkfaktoren der Intervention.

In einer ersten Studie wurde die Wirksamkeit der Integration von Elementen der Förderung selbstregulierten Lernens (Zielsetzung, Motivation, Lernstrategien, Selbstreflexion, volitionale Strategien) in eine curricular valide Unterrichtseinheit im Fach Naturwissenschaften überprüft. An der Studie nahmen 199 Schüler(innen) der siebten Jahrgangsstufe einer Gesamtschule teil. Die Evaluation zeigte, dass die Intervention zu einem Anstieg selbstregulierten Lernens in der Trainingsgruppe führte und dass dieser Vorteil gegenüber der Kontrollgruppe zeitlich stabil war. Trotz reduzierter Unterrichtszeit für fachbezogene Inhalte wurde unter der Bedingung mit Selbstregulationsanregungen fachlich ebenso viel gelernt wie in der Vergleichsgruppe. Im Follow-up lag die fachbezogene Leistung der trainierten Schüler(innen) sogar deutlich über der Leistung der Vergleichsgruppe. Differentielle Analysen ergaben, dass Schüler(innen) mit unterschiedlichen Ausgangsvoraussetzungen im selbstregulierten Lernen sowie Schüler(innen) mit Migrationshintergrund vergleichbar gut von der Förderung angesprochen wurden.

In einer zweiten Studie ging es um die Identifikation von selbstregulationsförderlichen Merkmalen der Instruktion, die unabhängig von strukturierten Trainingsprogrammen in den regulären Unterricht integriert werden können. Der Einfluss von Feedback und Bewertungsstandards auf die Selbstregulation und den Erwerb einer neuen mathematischen Fähigkeit wurde bei 90 Fünftklässler(inne)n untersucht. Die im Rahmen der Studie eingesetzte Form des graphisch dargestellten Feedbacks beeinflusste sowohl metakognitive Fähigkeiten als auch den Fähigkeitserwerb positiv. Die Ergebnisse deuteten zudem auf einen Einfluss von Bewertungsstandards auf motivationale Aspekte hin.

Die Arbeit dokumentiert, dass eine wirksame Förderung selbstregulierten Lernens im Unterrichtskontext auf verschiedenen Ebenen ansetzen und im Hinblick auf das Inhaltsspektrum variieren kann. Die spezifischen Herausforderungen und Chancen der beiden dargestellten Ansätze werden methodenkritisch diskutiert und Implikationen für die Praxis aufgezeigt. 


\section{Literatur}

Ames, C. \& Ames, R. (1978). The thrill of victory and the agony of defeat: Children's self and interpersonal evaluations in competitive and noncompetitive environments. Journal of Research and Development in Education, 12, 79-87.

Artelt, C. (1999). Lernstrategien und Schulerfolg - Eine handlungsnahe Studie. Zeitschrift für Pädagogische Psychologie und Entwicklungspsychologie, 31, 86-96.

Artelt, C. (2000). Wie prädiktiv sind Selbstberichte über den Gebrauch von Lernstrategien für strategisches Lernen? Zeitschrift für Pädagogische Psychologie, 14, 72-84.

Artelt, C., Baumert, J. \& Julius-McElvany, N. (2003). Selbstreguliertes Lernen: Motivation und Strategien in den Ländern der Bundesrepublik Deutschland. In J. Baumert, C. Artelt, E. Klieme, J. Neubrand, M. Prenzel, U. Schiefele, W. Schneider, K.-J. Tillmann \& M. Weiß (Hrsg.), PISA 2000: Ein differenzierter Blick auf die Länder der Bundesrepublik Deutschland (S. 131-164). Opladen: Leske \& Budrich.

Artelt, C. \& Schellhas, B. (1996). Zum Verhältnis von Strategiewissen und Strategieanwendung und ihren kognitiven und emotional-motivationalen Bedingungen im Schulalter. Empirische Pädagogik, 10, 277-305.

Bandura, A. (1969). Principles of behavior modification. New York: Holt, Rinehart \& Winston.

Bandura, A. (1977). Self-efficacy: Toward a unifying theory of behavioral change. Psychological Review, 84, 191-215.

Bandura, A. (1986). Social foundations of thoughts and action: A social cognitive theory. Englewood Cliffs, NJ: Prentice Hall.

Bandura, A. (1989). Human agency in social cognitive theory. American Psychologist, 44, $1175-1184$.

Bandura, A. (1991). Self-regulation and motivation through anticipatory and self-reactive mechanisms. In R. A. Dienstbier (Ed.), Nebraska Symposium on Motivation (Vol. 38, pp. 69-164). Lincoln: University of Nebraska Press.

Bandura, A. (1997). Self-efficacy: The exercise of control. New York: Erlbaum.

Bandura, A. \& Jourden, F. J. (1991). Self-regulatory mechanisms governing the impact of social comparison on complex decision making. Journal of Personality and Social Psychology, 6, 941-951. 
Bannert, M. (2005). Explorationsstudie zum spontanen metakognitiven Strategie-Einsatz in hypermedialen Lernumgebungen. In C. Artelt \& B. Moschner (Hrsg.), Lernstrategien und Metakognition: Implikationen für Forschung und Praxis (S. 129-153). Münster: Waxmann.

Baumert, J. (1993). Lernstrategien, motivationale Orientierungen und Selbstwirksamkeitsüberzeugungen im Kontext schulischen Lernens. Unterrichtswissenschaft, 4, 327-354.

Baumert, J., Klieme, E., Neubrand, J., Prenzel, M., Schiefele, U. \& Schneider, W. (2001). PISA 2000 - Basiskompetenzen von Schülerinnen und Schülern im internationalen Vergleich. Opladen: Leske \& Budrich.

Baumert, J. \& Köller, O. (1996). Lernstrategien und schulische Leistungen. In J. Möller \& O. Köller (Hrsg.), Emotionen, Kognitionen und Schulleistung (S. 137-154). Weinheim: Beltz.

Bloom, B. S. (1985). Developing talent in young people. New York, NY: Ballatine Books.

Blumenfeld, P., Fishman, B. J., Krajcik, J. \& Marx, R. W. (2000). Creating usable innovations in systemic reform: Scaling-up technology-embedded project-based science in urban schools. Educational Psychologist, 35, 149-164.

Boekaerts, M. (1999). Self-regulated learning: Where we are today. International Journal of Educational Research, 31, 445-457.

Boekaerts, M., \& Corno, L. (2005). Self-Regulation in the Classroom: A Perspective on Assessment and Intervention. Applied Psychology: An International Review, 54, 199231.

Bögeholz, S. (2006). Explizit Bewerten und Urteilen - Beispielkontext Streuobstwiese. Praxis der Naturwissenschaften. Biologie in der Schule, 55, 17-24.

Bögeholz, S. \& Barkmann, J. (2005): Rational choice and beyond: Handlungsorientierende Kompetenzen für den Umgang mit faktischer und ethischer Komplexität. In R. Klee, A. Sandmann \& H. Vogt (Hrsg.), Lehr- und Lernforschung in der Biologiedidaktik, Bd. 2 (S. 211-224). Innsbruck: Studienverlag.

Bol, L. \& Hacker, D. (2001). The effect of practice tests on students' calibration and performance. Journal of Experimental Education, 69, 133-151.

Bol, L., Hacker, D. J., O'Shea, P. \& Allen, D. (2005). The influence of overt practice, achievement level, and explanatory style on calibration accuracy and performance. Journal of Experimental Education, 73, 269-290.

Borkowski, J. G. (1992). Metacognitive theory: A framework for teaching literacy, writing, and math skills. Journal of Learning Disabilities, 25, 253-257. 
Borkowski, J. G., Carr, M., Rellinger, E. \& Pressley, M. (1990). Self-regulated cognition: Interdependence of metacognition, attributions, and self-esteem. In B. Jones \& L. Idol (Eds.), Dimensions of thinking and cognitive instruction (pp. 53-92). Hillsdale, NJ: Erlbaum.

Brannick, M. T., Miles, D. E. \& Kisamore, J. L. (2005). Calibration between student mastery and self-efficacy. Studies in Higher Education, 30, 473-483.

Brickman, P. \& Bulman, R. J. (1977). Pleasure and pain in social comparison. In J. M. Suls \& R. L. Miller (Eds.), Social comparison processes: Theoretical and empirical perspectives (pp. 149-186). Washington, DC: Hemisphere.

Butler, D. (1998). The strategic content learning approach to promoting self-regulated learning: A report of three studies. Journal of Educational Psychology, 90, 682-697.

Butler, D. L. \& Winne, P. H. (1995). Feedback and self-regulated learning: A theoretical synthesis. Review of Educational Research, 65, 245-281.

Chen, P. (2002). Exploring the accuracy and predictability of the self-efficacy beliefs of seventh-grade mathematics students. Learning and Individual Differences, 14, 79-92.

Chen, P. \& Zimmerman, B. J. (2007). A cross-national comparison study on the accuracy of self-efficacy beliefs of middle-school mathematics students. The Journal of Experimental Education, 75, 221-244.

Cleary, T., \& Zimmerman, B. J. (2001). Self-regulation differences during athletic practice by experts, non-experts, and novices. Journal of Applied Sport Psychology, 13, 61-82.

Cleary, T. J. \& Zimmerman, B. J. (2004). Self-regulation empowerment program: A schoolbased program to enhance self-regulated and self-motivated cycles of student learning. Psychology in the Schools, 41, 537-550.

Cleary, T. \& Zimmerman, B. J. (2006). Teacher's perceived usefulness of strategy microanalystic assessment information. Psychology in the Schools, 43, 149-155.

Cohen, J. (1988). Statistical power analysis for the behavioral sciences (2nd ed.). Hillsdale, NJ: Erlbaum.

Cronbach, L. J. (1957). The two disciplines of scientific psychology. American Psychologist, 12, 671-684.

den Elzen-Rump, V. \& Leutner, D. (2007). Naturwissenschaftliche Sachtexte verstehen - Ein computerbasiertes Trainingsprogramm für Schüler der 10. Jahrgangsstufe zum Selbstregulierten Lernen mit einer Mapping-Strategie. In M. Landmann \& B. Schmitz (Hrsg.), Selbstregulation erfolgreich fördern. Praxisnahe Trainingsprogramme für ein effektives Lernen (S. 251-268). Berlin: Kohlhammer. 
Dewey, J. (1916). Democracy and Education. An introduction to the philosophy of education. New York: Free Press.

Eggert, S. \& Bögeholz, S. (submitted). Students' Use of Decision Making Strategies with regard to Socio-Scientific Issues - An Application of the Partial Credit Model.

English, H. B. \& English, A. C. (1958). A comprehensive dictionary of psychological and psycholoanalytic terms. New York: David McCay Inc.

Ericsson, A. K. \& Lehman, A. C. (1996). Expert and exceptional performance: Evidence of maximal adaption to task constraints. Annual Review of Psychology, 47, 273-305.

Ewers, C. A. \& Wood, N. L. (1993). Sex and ability differences in children's math selfefficacy and prediction accuracy. Learning and Individual Differences, 5, 259-267.

Festinger, L. (1954). A theory of social comparison processes. Human Relations, 7, 117-140.

France-Kaatrude, A. \& Smith, W. P. (1985). Social comparison, task motivation, and the development of self-evaluative standards in children. Developmental Psychology, 21, 1080-1089.

Fuchs, L. S., Fuchs, D., Prentice, K., Burch, M., Hamlett, C. L., Owen, R. \& Schroeter, K. (2003). Enhancing third-grade students' mathematical problem solving with selfregulated learning strategies. Journal of Educational Psychology, 95, 306-315.

Glaser, C. \& Brunstein, J. (2007a). Förderung von Fertigkeiten zur Überarbeitung narrativer Texte bei Schülern der 6. Klasse. Effekte von Revisionsstrategien und selbstregulatorischen Prozeduren. Zeitschrift für Pädagogische Psychologie, 21, 51-63.

Glaser, C. \& Brunstein, J. (2007b). Improving fourth-grade students' composition skills: Effects of strategy instruction and self-regulation procedures. Journal of Educational Psychology, 99, 297-310.

Gräsel, C. \& Parchmann, I. (2004). Implementationsforschung - oder: Der steinige Weg, Unterricht zu verändern. Unterrichtswissenschaft, 32, 196-214.

Hacker, D. J., Bol, L., Horgan, D. \& Rakow, E. (2000). Test prediction and performance in a classroom context. Journal of Educational Psychology, 92, 160-170.

Hager, W. \& Hasselhorn, M. (1992). Memory monitoring and memory performance: Linked closely or loosely? Psychological Research, 54, 110-113.

Hasselhorn, M. (1992). Metakognition und Lernen. In G. Nold (Hrsg.), Lernbedingungen und Lernstrategien. Welche Rolle spielen kognitive Verstehensstrukturen? (S. 35-63). Tübingen: Narr.

Hasselhorn, M. (1996). Kategoriales Orientieren bei Kindern. Göttingen: Hogrefe. 
Hasselhorn, M. \& Gold, A. (2006). Pädagogische Psychologie: Erfolgreiches Lernen und Lehren. Stuttgart: Kohlhammer.

Hasselhorn, M. \& Hager, W. (1989). Prediction accuracy and memory performance: Correlational and experimental tests of a metamemory hypothesis. Psychological Research, $51,147-152$.

Hasselhorn, M. \& Labuhn, A. S. (2008). Metakognition und selbstreguliertes Lernen. In W. Schneider \& M. Hasselhorn (Hrsg.), Handbuch Pädagogische Psychologie (S. 28-37). Göttingen: Hogrefe.

Heller, K. A. \& Perleth, C. (2000). Kognitiver Fähigkeitstest für 4.-12. Klassen, Revision (KFT 4-12+R). Göttingen: Beltz.

Horgan, D. (1990). Competition, calibration, and motivation. Teaching Thinking and Problem Solving, 12, 5-10.

Kitsantas, A. \& Zimmerman, B. J. (1998). Self-regulation of motoric learning: A strategic cycle view. Journal of Applied Sport Psychology, 10, 220-239.

Kitsantas. A. \& Zimmerman, B. J. (2002). Comparing self-regulatory processes among novice, non-expert, and expert volleyball players: A micronanlytic study. Journal of Applied Sport Psychology, 13, 365-379.

Kitsantas, A. \& Zimmerman, B. J. (2006). Enhancing self-regulation of practice: The influence of graphing and self-evaluative standards. Metacognition and Learning, 1, 202212 .

Kitsantas, A., Zimmerman, B. J. \& Cleary, T. (2000). The role of observation and emulation in the development of athletic self-regulation. Journal of Educational Psychology, 91, $241-250$.

Klassen, R. M. (2002). A question of calibration: A review of the self-efficacy beliefs of students with learning disablilities. Learning Disability Quarterly, 25, 88-102.

Klassen, R. M. (2006). Too much confidence? The self-efficacy beliefs of adolescents with learning disabilities. In F. Pajares \& T. Urdan (Eds.), Self-efficacy beliefs of adolescents (pp. 181-200). Greenwich, CT: Information Age.

Klassen, R. M. (2007). Using predictions to learn about the self-efficacy of early adolescents with and without learning disablilities. Contemporary Educational Psychology, 32, $173-187$. 
Klauer, K.-J. (1997). Lässt sich die Strategie des induktiven Denkens auf schulisches Lernen transferierbar lehren? Zeitschrift für Entwicklungspsychologie und Pädagogische Psychologie, 29, 225-241.

Klieme, E. et al. (Hrsg.) (2003). Expertise zur Entwicklung nationaler Bildungsstandards. Berlin: BMBF.

Klieme, E. \& Stanat, P. (2002). Zur Aussagekraft internationaler Schulleistungsvergleiche Befunde und Erklärungsansätze am Beispiel von PISA. Bildung und Erziehung, 55, $24-44$.

Kohlberg, L. (1981). The philosophy of moral development: Moral stages and the idea of justice. San Francisco: Harper \& Row.

Kruger, J. \& Dunning, D. (1999). Unskilled and unaware of it: How difficulties in recognizing one's own incompetence lead to inflated self-assessments. Journal of Personality and Social Psychology, 77, 1121-1134.

Kuhl, J. (1985). Volitional mediators of cognitive behavior consistency: Self-regulatory processes and action versus state orientation. In J. Kuhl and J. Beckman (Eds.), Action control: From cognition to behavior (pp. 101-128). New York: Springer.

Labuhn, A. S., Bögeholz, S. \& Hasselhorn, M. (2008). Lernförderung durch Anregung der Selbstregulation im naturwissenschaftlichen Unterricht, Zeitschrift für Pädagogische Psychologie, 22, 13-24.

Labuhn, A. S., Bögeholz, S. \& Hasselhorn, M. (in Druck). Überprüfung der langfristigen und differentiellen Wirksamkeit der Selbstregulationsförderung im naturwissenschaftlichen Unterricht. Zeitschrift für Entwicklungspsychologie und Pädagogische Psychologie.

Labuhn, A. S., Zimmerman, B. J. \& Hasselhorn, M. (submitted). Enhancing students' selfregulation and mathematics performance: The influence of feedback and selfevaluative standards.

Leopold, C., den Elzen-Rump, V. \& Leutner, D. (2006). Selbstreguliertes Lernen aus Sachtexten. In M. Prenzel \& L. Allolio-Näcke (Hrsg.), Untersuchungen zur Bildungsqualität von Schule. Abschlussbericht des DFG-Schwerpunktprogramms (S. 268-288). Münster: Waxmann.

Leopold, C. \& Leutner, D. (2002). Der Einsatz von Lernstrategien in einer konkreten Lernsituation bei Schülern unterschiedlicher Jahrgangsstufen. Zeitschrift für Pädagogik, 45, 240-258.

Lillard, P. P. (1972). Montessori: A modern approach. New York: Schocken Books. 
Locke, E. A., \& Latham, G. P. (1990). A theory of goal setting and task performance. Englewood Cliffs: Prentice Hall.

Meichenbaum, D. (1977). Cognitive-behavior modification: An integrative approach. New York: Plenum.

Montessori, M. (1964). The absorbent mind. Wheaton, IL: Theosophical Press.

Mullis, I. V. S., Martin, M. O., Gonzalez, E. J. \& Chrostowski, S. J. (2004). Findings From IEA's Trends in International Mathematics and Science Study at the Fourth and Eighth Grades. Chestnut Hill, MA: TIMSS \& PIRLS International Study Center, Boston College.

Nietfeld, J. L. \& Schraw, G. (2002). The effect of knowledge and strategy training on monitoring accuracy. The Journal of Educational Research, 95, 131-142.

OECD (2004). Learning for Tomorrow's World: First Results from PISA 2003. Programme for International Student Assessment.

Pajares, F. (1996). Self-efficacy in academic settings. Review of Educational Research, 66, 543-578.

Pajares, F. (2008). Motivational role of self-efficacy beliefs in self-regulated learning. In D. H. Schunk \& B. J. Zimmerman (Eds.), Motivation and self-regulated learning: Theory, research, and applications (pp. 111-139). New York: Lawrence Erlbaum Associates.

Pajares, F. \& Graham, L. (1999). Self-efficacy, motivation constructs, and mathematics performance of entering middle school students. Contemporary Educational Psychology, $24,124-139$.

Pajares, F. \& Kranzler, J. (1995). Self-efficacy beliefs and general mental ability in mathematical problem-solving. Contemporary Educational Psychology, 20, 426-443.

Pajares, F. \& Miller, M. D. (1994). The role of self-efficacy and self-concept beliefs in mathematical problem-solving: A path analysis. Journal of Educational Psychology, 86, 193-203.

Pajares, F. \& Miller, M. D. (1997). Mathematics self-efficacy and mathematical problem solving: implications of using different forms of assessment. The Journal of Experimental Education, 65, 213-228.

Perels, F., Dignath, C. \& Schmitz, B. (in Druck). Is it possible to improve mathematical achievement by means of self-regulation strategies? Evaluation of an intervention in regular math classes. European Journal of Psychology of Education. 
Perels, F., Gürtler, T. \& Schmitz, B. (2005). Training of self-regulatory and problem-solving competence. Learning and Instruction, 15, 123-139.

Perels, F., Otto, B., Landmann, M., Hertel, S. \& Schmitz, B. (2007). Self-regulation from a process perspective. Journal of Psychology, 215, 194-204.

Perels, F., Schmitz, B. \& Bruder, S. (2003). Trainingsprogramm zur Förderung der Selbstregulationskompetenz von Schülern der achten Gymnasialklasse. Unterrichtswissenschaft, 31, 23-37.

Piaget, J. (1962). Play, dreams and imitation. New York: Norton.

Pintrich, P. R. (2000). The role of goal orientation in self-regulated learning. In M. Boekaerts, P. R. Pintrich \& M. Zeidner (Eds.), Handbook of Self-Regulation (pp. 451-502). San Diego, CA: Academic Press.

Pintrich, P. R. \& De Groot, E. V. (1990). Motivational and self-regulated learning components of classroom academic learning. Journal of Educational Psychology, 82, 33-40.

Prenzel, M., Artelt, C., Baumert, J., Blum, W., Hammann, M., Klieme, E. \& Pekrun, R. (Hrsg.) (2007). PISA 2006. Die Ergebnisse der dritten internationalen Vergleichsstudie. Münster: Waxmann.

Prenzel, M., Baumert, J., Blum, W., Lehmann, R., Leutner, D., Neubrand, M., Pekrun, R., Rost, J. \& Schiefele, U. (Hrsg.) (2006). PISA 2003. Untersuchungen zur Kompetenzentwicklung im Verlauf eines Schuljahres. Münster: Waxmann.

Pressley, M., Woloshyn, V., Lysynchuk, L. M., Martin, V., Wood, E. \& Willoughby, T. (1990). A primer of research on cognitive strategy instruction: The important issues and how to address them. Educational Psychology Review, 2, 1-58.

Rost, D. H. (2007). Interpretation und Bewertung pädagogisch-psychologischer Studien (2. Aufl.). Weinheim: Beltz.

Rotter, J. B. (1954). Social learning and clinical psychology. New York: Prentice Hall.

Schmitz, B. (1990). Univariate and multivariate time-series models: The analysis of intraindividual variability and intraindividual relationships. In A. v. Eye (Ed.), Statistical methods in longitudinal research, Vol. II: Time series and categorical longitudinal data (pp. 351-386). New York: Academic Press.

Schmitz, B. (2001). Self-Monitoring zur Unterstützung des Transfers einer Schulung in Selbstregulation für Studierende: Eine prozessanalytische Untersuchung. Zeitschrift für Pädagogische Psychologie, 15, 181-197.

Schmitz, B. (2006). Advantages of studying processes in educational research. Learning and Instruction, 16, 233-499. 
Schmitz, B. \& Skinner, E. A. (1993). Perceived control, effort, and academic performance: Interindividual, intraindividual, and multivariate time series analysis. Journal of Personality and Social Psychology, 64, 1010-1028.

Schmitz, B. \& Wiese, B. S. (2006). New perspectives for the evaluation of training sessions in self-regulated learning: Time-series analyses of diary data. Contemporary Educational Psychology, 31, 64-96.

Schraw, G., Potenza, M. T. \& Nebelsick-Gullet, L. (1993). Constraints on the calibration of performance. Contemporary Educational Psychology, 18, 455-463.

Schreblowski, S. \& Hasselhorn, M. (2001). Zur Wirksamkeit zusätzlicher Motivationskomponenten bei einem metakognitiven Textverarbeitungstraining. Zeitschrift für Pädagogische Psychologie, 15, 145-154.

Schunk, D. H. (1982). Verbal self-regulation as a facilitator of children's achievement and self-efficacy. Human Learning, 1, 265-277.

Schunk, D. H. (1986). Verbalization and children's self-regulated learning. Contemporary Educational Psychology, 11, 347-369.

Schunk, D. H. (1994). Self-regulation of self-efficacy and attributions in academic settings. In D. H. Schunk \& B. J. Zimmerman (Eds.), Self-regulation of learning and performance: Issues and educational implications (pp. 75-99). Hillsdale, NJ: Erlbaum.

Schunk, D. H. (1996). Goal and self-evaluative influences during children's cognitive skill learning. American Educational Research Journal, 33, 359-382.

Schunk, D. H. \& Ertmer, P. A. (2000). Self-regulation and academic learning: Self-efficacy enhancing interventions. In M. Boekaerts, P. R. Pintrich \& M. Zeidner (Eds.), Handbook of self-regulation (pp. 631-651). San Diego, CA: Academic Press.

Schunk, D. H. \& Pajares, F. (2004). Self-efficacy in education revisited. Empirical and applied evidence. In D. M. McInerney \& S. van Etten (Eds.), Big theories revisited (pp. 115-138). Greenwich, CT: Information Age Publishing.

Schunk, D. H. \& Rice, J. M (1993). Strategy fading and progress feedback: Effects on selfefficacy and comprehension among students receiving remedial reading services. The Journal of Special Education, 27, 257-276.

Schunk, D. H. \& Schwartz, C. W. (1993). Goals and progress feedback: Effects on selfefficacy and writing achievement. Contemporary Educational Psychology, 18, 337354. 
Schunk, D. H. \& Zimmerman, B. J. (1998). Conclusions and future directions for academic interventions. In D. H. Schunk \& B. J. Zimmerman (Eds.), Self-regulated learning: From teaching to self-reflective practice (pp. 225-235). New York: Guilford.

Selman, R. L. (1980). The Growth of Interpersonal Understanding - Developmental and Clinical Analyses. New York: Academic Press.

Souvignier, E. \& Antoniou, F. (2007). Förderung des Leseverständnisses bei Schülerinnen und Schülern mit Lernschwierigkeiten - eine Metaanalyse. Vierteljahresschrift für Heilpädagogik und ihre Nachbargebiete, 76, 46-62.

Souvignier, E. \& Mokhlesgerami, J. (2006). Using self-regulation as a framework for implementing strategy-instruction to foster reading comprehension. Learning and Instruction, 16, 57-71.

Souvignier, E. \& Trenk-Hinterberger, I. (2007). Ein Drei-Stufen-Modell zur Implementation neuer Unterrichtskonzepte in den Schulalltag. In M. Krämer, S. Preiser \& K. Brusdeylins (Hrsg.), Psychologiedidaktik und Evaluation (S. 197-206). Göttingen: Vandenhoeck \& Ruprecht.

Spörer, N. \& Brunstein, J.C. (2006). Erfassung selbstregulierten Lernens mit Selbstberichtsverfahren. Ein Überblick zum Stand der Forschung. Zeitschrift für Pädagogische Psychologie, 20, 147-160.

Staemmler, D. (2006). Lernstile und interaktive Lernprogramme. Deutscher Universitätsverlag.

Stone, N. J. (2000). Exploring the relationship between calibration and self-regulated learning. Educational Psychology Review, 12, 437-475.

Thompson, T. \& Musket, S. (2005). Does priming for mastery goals improve the performance of students with an entity view of ability? British Journal of Educational Psychology, 75, 391-409.

Thompson, T. \& Perry, Z. (2005). Is the poor performance of self-worth protective students linked with social comparison goals? Educational Psychology, 25, 471-490.

Thorndike, E. L. (1932). The fundamentals of learning. New York: Teachers College Press.

Veenman, M. V. J. \& Beishuizen, J. J. (2004). Intellectual and metacognitive skills of novices while studying texts under conditions of text difficulty and time constraint. Learning and Instruction, 14, 621-640.

Weiner, B. (1979). A theory of motivation for some classroom experiences. Journal of Educational Psychology, 71, 3-25. 
Weinert, F. E. (2001). Vergleichende Leistungsmessung in Schulen - eine umstrittene Selbstverständlichkeit. In F. E. Weinert (Hrsg.), Leistungsmessungen in Schulen (S. 17-31). Weinheim: Beltz.

Weinstein, C. E. \& Mayer, R. E. (1986). The teaching of learning strategies. In M. C. Wittrock (Ed.), Handbook on research on teaching (pp. 315-327). New York: Macmillan.

Winne, P. H. (1997). Experimenting to bootstrap self-regulated learning. Journal of Educational Psychology, 89, 397-410.

Winne, P. H. (2000). Information processing models of self-regulated learning. In B. J. Zimmerman \& D. H. Schunk (Eds.), Self-regulated learning and academic achievement: Theory, research, and practice. New York: Longman.

Winne, P. H., \& Hadwin, A. F. (1998). Studying as self-regulated learning. In D. J. Hacker, J. Dunlosky, \& A. C. Graesser (Eds.), Metacognition in educational theory and practice (pp. 279-306). Hillsdale, NJ: Erlbaum.

Winne, P. H. \& Jamieson-Noel, D. (2002). Exploring students' calibration of self-reports about study tactics and achievement. Contemporary Educational Psychology, 27, 551572 .

Wood, J. V. (1989). Theory and reserach concerning social comparisons of personal attributes. Psychological Bulletin, 106, 231-248.

Wood, E., Woloshyn, V. E. \& Willoughby, T. (1995). Cognitive strategy instruction for middle and high schools. Cambridge, MA: Brookline Books.

Yates, J. F. (1990). Judgment and decision making. Englewood Cliffs, NJ: Prentice Hall.

Zeidner, M., Boekaerts, M. \& Pintrich, P. R. (2000). Self-regulation. Directions and challenges for future research. In M. Boekaerts, P. R. Pintrich \& M. Zeidner (Eds.), Handbook of self-regulation (pp. 749-768). San Diego, CA: Academic Press.

Zimmerman, B. J. (1989a). A social cognitive view of self-regulated learning. Journal of Educational Psychology, 82, 297-306.

Zimmerman, B. J. (1989b). Models of self-regulated learning and academic achievement. In B. J. Zimmerman \& D. H. Schunk (Eds.), Self-regulated learning and academic achievement: Theory, research, and practice (pp. 1-25). New York: Springer.

Zimmerman, B. J. (1990). Self-regulated learning and academic achievement: An overview. Educational Psychologist, 25, 3-17. 
Zimmerman, B. J. (1994). Dimensions of academic self-regulation: A conceptual framework for education. In Schunk, D. H., \& Zimmerman, B. J. (Eds.), Self-Regulation of Learning and Performance: Issues and Educational Applications (pp. 3-21). Hillsdale, NJ: Lawrence Erlbaum Associates.

Zimmerman, B. J. (1995a). Self-efficacy and educational development. In A. Bandura (Ed.), Self-efficacy in changing societies (pp. 202-231). New York: Cambridge University Press.

Zimmerman, B. J. (1995b). Self-regulation involves more than metacognition: A socialcognitive perspective. Educational Psychologist, 29, 217-221.

Zimmerman, B. J. (2000). Attaining self-regulation: A social cognitive perspective. In M. Boekaerts, P. R. Pintrich \& M. Zeidner (Eds.), Handbook of self-regulation (pp. 1339). San Diego, CA: Academic Press.

Zimmerman, B. J. (2002). Becoming a self-regulated learner: An overview. Theory into Practice, 41, 64-72.

Zimmerman, B. J. (2006). Enhancing students' academic responsibility and achievement. A social-cognitive self-regulatory account. In R. J. Sternberg \& R. F. Subotnik (Eds.), Optimizing student success in school with the other three Rs: Reasoning, resilience, and responsibility (pp. 179-197). Charlotte, NC: Information Age Publishing, Inc.

Zimmerman, B. J. \& Bandura, A. (1994). Impact of self-regulatory influences on writing course attainment. American Educational Research Journal, 31, 845-862.

Zimmerman, B. J. \& Campillo, M. (2003). Motivating self-regulated problem solvers. In J.E Davidson \& R. Sternberg (Eds.), The psychology of problem solving (pp. 233-263). New York: Cambridge University Press.

Zimmerman, B. J. \& Kitsantas, A. (1996). Self-regulated learning of a motoric skill: The role of goal setting and self-monitoring. Journal of Applied Sport Psychology, 8, 69-84.

Zimmerman, B. J. \& Kitsantas, A. (1997). Developmental phases in self-regulation: Shifting from process goals to outcome goals. Journal of Educational Psychology, 89, 29-36.

Zimmerman, B. J. \& Kitsantas, A. (1999). Acquiring writing revision skill: Shifting from process to outcome self-regulatory goals. Journal of Educational Psychology, 91, 241-250.

Zimmerman, B. J. \& Martinez-Pons, M. (1986). Development of a structural interview for assessing student use of self-regulated learning strategies. American Educational Research Journal, 23, 614-628. 
Zimmerman, B. J. \& Martinez-Pons, M. (1988). Construct validation of a strategy model of student self-regulated learning. Journal of Educational Psychology, 80, 284-290.

Zimmerman, B. J. \& Martinez-Pons, M. (1990). Student differences in self-regulated learning: Relating grade, sex, and giftedness to self-efficacy and stragegy use. Journal of Educational Psychology, 82, 51-59.

Zimmerman, B. J. \& Ramdass, D. (submitted for review). Investigating the effects of strategy training on the self-evaluative judgments of middle school students. 
Anhang 


\section{Anhang A}

\section{Messinstrument von Studie 1}

(Fragebogen aus der Follow-up-Erhebung) 
Hallo!

Vor einiger Zeit hast Du wahrscheinlich schon einmal einen solchen Fragebogen ausgefüllt.

Heute bitten wir Dich erneut, die Fragen zu beantworten. Viele Fragen kommen Dir bestimmt noch bekannt vor, das macht aber nichts. Kreuze einfach die Antworten an, die Deiner Meinung nach am besten passen.

Vielen Dank für Deine Mitarbeit!

Bitte lies die Anleitungen und Fragen gut durch, bevor Du antwortest!

Hier ist ein Beispiel:

\begin{tabular}{lcccc} 
& $\substack{\text { stimmt gar } \\
\text { nicht } \\
\downarrow}$ & $\begin{array}{c}\text { stimmt eher } \\
\text { nicht } \\
\downarrow\end{array}$ & $\begin{array}{c}\text { stimmt ener } \\
\downarrow\end{array}$ & $\begin{array}{c}\text { stimmt genau } \\
\downarrow\end{array}$ \\
\hline Ich esse gerne Schokolade. & 0 & 0 & 0 & $\otimes$
\end{tabular}

Hier müsstest Du „stimmt genau“ ankreuzen, wenn Du sehr gerne Schokolade isst. Dies ist im Beispiel der Fall. Wenn Du überhaupt keine Schokolade magst, müsstest Du „stimmt gar nicht" ankreuzen. Wenn Du Schokolade eher nicht magst, müsstest Du „stimmt eher nicht“ ankreuzen; wenn Du Schokolade eher gerne magst, steht Dir die Antwort „stimmt eher" zur Verfügung.

Bevor Du anfängst, gib bitte noch einige Informationen zu Deiner Person an!

\begin{tabular}{|l|l|}
\hline Name: & \\
\hline Klasse: & \\
\hline Alter: & \\
\hline Bist Du ein Mädchen oder ein Junge? & $\square$ Mädchen $\square$ Junge \\
\hline
\end{tabular}


Es folgen jetzt einige Fragen zu dem Thema „Ernährung" aus dem NW-Unterricht. Dieses Thema habt Ihr im vergangenen Schuljahr behandelt. Wir möchten wissen, wie viel Du davon behalten hast.

Denke gut nach, vielleicht fallen Dir die Antworten ja noch ein.

\section{Nenne die drei lebenswichtigen Nährstoffe}

1. 2 . , 3.

Bei diesen Fragen gibt es immer nur eine richtige Antwort! Du musst also nur ein Kreuzchen bei der Antwort machen, die Du für richtig hältst.

2. Was sind die Konsequenzen einer mangelnden Energieversorgung durch Nahrungsaufnahme?

O Leistungsmangel \& Einschränkung der Organfunktionen

O Leistungssteigerung \& Müdigkeit

Bluthochdruck \& Sauerstoffmangel

O Leistungsmangel \& Konzentrationssteigerung

\section{Welche Aufgaben haben Kohlenhydrate?}

- Sie werden für den Abbau von Fett benötigt. Sie bringen Energie für Zellen und Nerven und sie helfen beim Aufbau von Knochen und Knorpel

S Sie sind wichtig für den Aufbau und den Erhalt von Zellen in Haut, Muskeln, Organen. Sie bilden Hormone und Enzyme.

S Sie tragen dazu bei, dass der Blutdruck sinkt und wir nicht soviel Schlaf benötigen

Sie sorgen für einen ausgeglichenen Vitaminhaushalt. Es reichen schon kleine Mengen aus. Sie müssen nicht täglich mit der Nahrung zugeführt werden.

\section{Was sind die wichtigsten Aufgaben des Eiweiß/der Proteine?}

Aufbau und Erhalt von Zellen in Haut, Muskeln, Organen, Bildung von Hormonen und Enzymen

- Herstellung eines ausgeglichenen Vitaminhaushalts, Förderung von Kreativität, Umwandlung von Fetten in Vitamine

Abbau von Gift- und Abfallstoffen im Körper, Schutz vor Bakterien, Stärkung der Abwehrkräfte

O Aufbau von Magensäure zur Verdauung von Speisen, Speichelproduktion, Abbau von Zellen

5. Was sind Mineralstoffe/Spurenelemente?

\begin{tabular}{cl}
\hline O & unlösliche Bestandteile der Nahrung \\
\hline W & wasserlösliche Substanzen \\
\hline Energieträger, die das Nahrungsvolumen verringern \\
\hline O eine Art von Fetten \\
\hline
\end{tabular}


6. Welche Stoffe gehören zu den Spurenelementen?

O Hefe, Aromastoffe, Vollkornmehl, Emulgator

○ Salzsäure, Traubenzucker, Galaktose, Silber

Eisen, Kupfer, Zink, Jod

○ Rapsöl, Sonnenblumenöl, Lebertran, Butter

\section{Welche Stoffe gehören zu den Mineralstoffen?}

O Kalium, Calcium, Natrium, Phosphat

○ Rapsöl, Sonnenblumenöl, Lebertran, Butter

O Vitamin B, Vitamin C, Beta-Carotin, Folsäure

O Stickstoff, Laktose, Traubenzucker, Fructose

\section{Was sind Vitamine?}

Stoffe, die für die Farbe im Obst verantwortlich sind. Um ihre positive Wirkung auf die Gesundheit zu entfalten, muss man täglich mindestens $100 \mathrm{~g}$ davon zu sich nehmen.

O Nahrungsbestandteile, die Hormone und Enzyme bilden. Ihr Speicher ist nur begrenzt.

- Wirkstoffe, die im Körper benötigt werden. Es reichen schon kleine Mengen aus. Sie müssen täglich mit der Nahrung zugeführt werden.

O Stoffe in der Nahrung, die für den speziellen Geschmack der einzelnen Lebensmittel zuständig sind.

\section{Welchen Sinn machen künstliche Vitaminpräparate?}

Sie sind sehr sinnvoll, da man nie genug Vitamine zu sich nehmen kann.

O Zum Anreichern in Zeiten erhöhten Energiebedarfs sind sie sinnvoll, sie machen aber keine Ernährungsfehler gut.

O Sie haben gar keinen Sinn, da es sich bei solchen Vitaminpräparaten nicht um echte Vitamine handelt.

O Man sollte sie auf jeden Fall vermeiden, da sie giftig sind.

10. Zum Leben wird jeden Tag Energie benötigt. Woraus setzt sich der Gesamtumsatz des Menschen zusammen?

O Grundumsatz + Leistungsumsatz

O Leistungsumsatz + Ruhepotential

O Grundumsatz + Körpergröße

O Leistungsumsatz + Kalorienzufuhr 
11. Welche Reihenfolge ist die richtige, wenn die Lebensmittel aufsteigend nach ihrem Energiegehalt pro $100 \mathrm{~g}$ geordnet werden sollen?

\section{O Vollkornbrot - Apfel - Butter - Schokolade}

O Apfel - Vollkornbrot - Schokolade - Butter

Schokolade - Butter - Apfel - Vollkornbrot

Butter - Schokolade - Apfel - Vollkornbrot

12. Was passiert im Körper, wenn man mehr Energie in Form von Nahrung zu sich nimmt als man verbraucht?

O Das Zuviel wird in Form von Fett gespeichert.

O Man kann sich besser konzentrieren und bessere Leistungen erbringen.

O Die Sehfähigkeit verschlechtert sich.

Os werden hauptsächlich Muskeln aufgebaut. 
Nun kommt noch ein Lückentext zum Thema Ernährung. Einige wichtige Begriffe fehlen, diese sollst Du in die Lücken einsetzen. In jede Lücke kommt nur ein Wort. Bitte lies den Text aufmerksam!

\section{Essen und Trinken}

Könntest Du Dir vorstellen eine riesige Pizza und zum Nachtisch ein leckeres Eis zu verspeisen? Sicherlich wärst Du danach gesättigt - aber würde diese Zusammenstellung auch Deine fördern?

Durch unser(e) können wir ganz wesentlich dazu beitragen, dass wir gesund bleiben.

Wenn die Nahrung täglich ca. $60 \%$ $25-30 \%$

$12-15 \%$ und mindestens

Liter Flüssigkeit enthält, ist der Bedarf eines Jugendlichen am besten gedeckt.

Auch die richtige Verteilung der Mahlzeiten trägt zum Wohlbefinden bei. Ganz besonders wichtig ist der Start in den Tag. In Vollkornbrot und Müsli stecken viele Die stellen die wichtigste

Energiequelle für körperliche Bewegung und Gehirntätigkeit dar.

In der Pause am Vormittag sollten ein Stück Obst oder knackiges rohes Gemüse wie z.B. Möhre oder Paprika nicht fehlen. Solche Lebensmittel enthalten wichtige Mineralstoffe und und sind leicht bekömmlich.

Bei den Hauptmahlzeiten sollte man auf Abwechslung achten. Es wird außerdem empfohlen, lieber Mahlzeiten über den Tag verteilt zu essen, anstatt bei einer Mahlzeit riesige Mengen zu verdrücken.

Häufig vergisst man, dass der Körper auch genügend Flüssigkeit pro Tag braucht, um Kreislauf und aufrecht zu halten.

Jeder Mensch kann durch sein Essverhalten dazu beitragen, ein Gewicht zu erreichen, bei dem er sich wohl fühlt und seiner Gesundheit nicht schadet. Doch in den Modezeitschriften und in der Werbung werden nur superschlanke Models gezeigt, denen viele junge Leute nacheifern.

Schlankheit wird oft gleichgesetzt mit

Häufig machen Jugendliche daher Radikaldiäten. Das ist aber keinesfalls gesund und kann dem Körper sehr schaden. Außerdem nehmen viele Menschen nach der Diät wieder zu und wiegen dann sogar eher als vorher.

Wer schlank werden oder bleiben möchte, sollte nicht zu viele fettreiche Lebensmittel essen, denn der häufige Verzehr von Lebensmitteln mit viel Fett kann zu führen.

Regelmäßige verbraucht zusätzlich Energie und verhindert eine Gewichtszunahme.

Das Wichtigste ist aber, dass sich jeder Mensch mit seinem Körpergewicht wohl fühlt. 
In NW hast Du im letzten Schuljahr auch einiges über das Bewerten von verschiedenen Sachen gelernt. Erinnerst Du Dich? Versuche bitte die Fragen zu beantworten.

\section{Wobei kann Bewertung nicht helfen?}

O bei der Entscheidung für ein Haustier

O bei der Auswahl einer Arbeitsgemeinschaft

O beim Fremdsprachenlernen

O bei der Entscheidung für ein Handy

\section{Finde die richtige Reihenfolge der Schritte einer Bewertung!}

\begin{tabular}{|c|c|}
\hline 0 & $\begin{array}{l}\text { Punktvergabe und Gewichtung - Kriterien festlegen, die für die Entscheidung wichtig sind - Multiplikation der Ge- } \\
\text { wichtung und der Punkte - Auswahl einer Option - Addition der Werte jeder Spalte }\end{array}$ \\
\hline 0 & $\begin{array}{l}\text { Kriterien festlegen, die für die Entscheidung wichtig sind - Punktvergabe und Gewichtung - Multiplikation der Ge- } \\
\text { wichtung und der Punkte - Addition der Werte jeder Spalte - Auswahl einer Option }\end{array}$ \\
\hline 0 & $\begin{array}{l}\text { Auswahl einer Option - Punktvergabe und Gewichtung - Multiplikation der Gewichtung und der Punkte - Addition } \\
\text { der Werte jeder Spalte - Kriterien festlegen, die für die Entscheidung wichtig sind }\end{array}$ \\
\hline 0 & $\begin{array}{l}\text { Kriterien festlegen, die für die Entscheidung wichtig sind - Punktvergabe und Gewichtung - Addition der Werte jeder } \\
\text { Spalte - Multiplikation der Gewichtung und der Punkte - Auswahl einer Option }\end{array}$ \\
\hline
\end{tabular}

\section{Welches Bewertungskriterium hilft nicht bei der Auswahl einer Apfelsorte?}

\begin{tabular}{cl}
\hline O & Farbe \\
\hline O Name einer Apfelsorte \\
\hline O & Geschmack \\
\hline O Vitamingehalt \\
\hline
\end{tabular}

4. Wie viele Kriterien muss man haben, um bewerten zu können?

\begin{tabular}{cl}
\hline O & mehr als ein Kriterium \\
\hline O & maximal 5 Kriterien \\
\hline O man braucht gar kein Kriterium \\
\hline O maximal 10 Kriterien \\
\hline
\end{tabular}




\section{Bei welcher Aussage handelt es sich um eine Gewichtung?}

○ Ich mag am liebsten süße Äpfel und deshalb bekommen alle süßen Äpfel die meisten Punkte.

O Ich nehme den Apfel „Boskop“, weil er die meisten Punkte bekommen hat.

O Der Geschmack ist mir bei einem Apfel zwei Mal so wichtig wie der Preis.

Ich bringe die Apfelsorten je nach erreichter Punktzahl in eine Rangfolge.

6. Warum sollte man für eine Bewertung erstmal Kriterien festlegen, die einem wichtig sind?

O damit man nicht falsch rechnet

- weil man sich so die eigenen Vorlieben klar machen kann

O damit man mehr Kriterien hat als die anderen aus der Klasse

- weil man sich dann auch falsch entscheiden darf

\section{Was sagst Du zu der folgenden Aussage:}

„Wenn man eine Entscheidung durch Bewertung getroffen hat, kann man sich sicher sein, dass auch alle andere Personen mit dieser Entscheidung übereinstimmen"?

Stimmt, weil man durch Bewertung alle Kriterien richtig berücksichtigt.

- Stimmt nicht, weil andere Personen anders bewerten können und damit zu anderen Entscheidungen kommen könnten.

O Stimmt, weil jeder die einzelnen Bewertungsschritte gut nachvollziehen kann.

S Stimmt, weil man bei einer Bewertung immer zum richtigen Ergebnis kommt. 
Im folgenden Abschnitt geht es um's Lernen und Arbeiten. Bitte beantworte die Fragen ehrlich und kreuze die Antwort an, die Deiner Meinung nach am besten passt.

\begin{tabular}{|c|c|c|c|c|}
\hline & $\begin{array}{c}\text { stimmt gar } \\
\text { nicht } \\
\downarrow\end{array}$ & $\begin{array}{c}\text { stimmt eher } \\
\text { nicht } \\
\downarrow\end{array}$ & $\underset{\downarrow}{\stackrel{s}{*}+i m m t}$ eher & $\underset{\downarrow}{\text { stimmt genau }}$ \\
\hline $\begin{array}{l}\text { Alle möglichen Gedanken lassen mir einfach keine Ruhe zum } \\
\text { Arbeiten. }\end{array}$ & 0 & $\mathrm{O}$ & O & 0 \\
\hline $\begin{array}{l}\text { Bei schwierigen Aufgaben kann ich eine große Ausdauer entwi- } \\
\text { ckeln. }\end{array}$ & O & O & O & $\mathrm{O}$ \\
\hline $\begin{array}{l}\text { Ich behalte mein Ziel im Auge und lasse mich nicht vom Weg ab- } \\
\text { bringen. }\end{array}$ & 0 & $\mathrm{O}$ & $\mathrm{O}$ & $\mathrm{O}$ \\
\hline $\begin{array}{l}\text { Ich kann es verhindern, dass meine Gedanken ständig von der } \\
\text { Aufgabe abschweifen. }\end{array}$ & 0 & O & $\mathrm{O}$ & 0 \\
\hline $\begin{array}{l}\text { Ich kann mich lange Zeit auf eine Sache konzentrieren, wenn es } \\
\text { nötig ist. }\end{array}$ & 0 & 0 & O & O \\
\hline Ich kann sehr hartnäckig sein, wenn ich etwas erreichen möchte. & 0 & O & O & O \\
\hline Ich verfolge oft Ziele, die besonders anspruchsvoll sind. & $\mathrm{O}$ & $\mathrm{O}$ & $\mathrm{O}$ & $\mathrm{O}$ \\
\hline $\begin{array}{l}\text { Nach einer Unterbrechung kann ich mich leicht wieder konzentrie- } \\
\text { ren. }\end{array}$ & 0 & $\mathrm{O}$ & $\mathrm{O}$ & $\mathrm{O}$ \\
\hline $\begin{array}{l}\text { Wenn bei der Lösung eines Problems Schwierigkeiten auftreten, } \\
\text { strenge ich mich mehr an. }\end{array}$ & 0 & $\mathrm{O}$ & O & $\mathrm{O}$ \\
\hline Wenn es nötig ist, kann ich meine Gefühle unter Kontrolle bringen. & 0 & $\mathrm{O}$ & O & $\mathrm{O}$ \\
\hline $\begin{array}{l}\text { Wenn ich eine neuartige Aufgabenstellung bearbeite, finde ich } \\
\text { meist Mittel und Wege, diese zu lösen. }\end{array}$ & 0 & 0 & 0 & 0 \\
\hline $\begin{array}{l}\text { Wenn ich etwas für die Schule zu erledigen habe, fällt es mir oft } \\
\text { schwer anzufangen. }\end{array}$ & O & 0 & O & 0 \\
\hline $\begin{array}{l}\text { Wenn ich etwas Schwieriges zu lernen habe, beginne ich lieber } \\
\text { gleich damit, anstatt es aufzuschieben. }\end{array}$ & 0 & O & $\mathrm{O}$ & 0 \\
\hline $\begin{array}{l}\text { Wenn ich für die Schule etwas Unangenehmes zu erledigen habe, } \\
\text { bringe ich es schnell hinter mich. }\end{array}$ & 0 & 0 & 0 & 0 \\
\hline $\begin{array}{l}\text { Wenn ich mir einmal ein Ziel gesetzt habe, lasse ich mich nicht } \\
\text { leicht davon abbringen. }\end{array}$ & 0 & 0 & 0 & 0 \\
\hline $\begin{array}{l}\text { Wenn ich mir einmal etwas in den Kopf gesetzt habe, lasse ich } \\
\text { mich auch durch große Schwierigkeiten nicht davon abbringen. }\end{array}$ & 0 & 0 & 0 & 0 \\
\hline $\begin{array}{l}\text { Wenn ich mir vorgenommen habe zu lernen, beginne ich damit so } \\
\text { schnell wie möglich. }\end{array}$ & 0 & 0 & 0 & 0 \\
\hline $\begin{array}{l}\text { Wenn ich Sorgen habe, kann ich mich nicht auf eine Tätigkeit } \\
\text { konzentrieren. }\end{array}$ & 0 & 0 & 0 & 0 \\
\hline
\end{tabular}




\begin{tabular}{|c|c|c|c|c|}
\hline & $\begin{array}{c}\text { stimmt gar } \\
\text { nicht } \\
\downarrow\end{array}$ & $\begin{array}{c}\text { stimmt eher } \\
\text { nicht } \\
\downarrow\end{array}$ & $\underset{\downarrow}{\operatorname{stimmt~eher~}}$ & $\underset{\downarrow}{\text { stimmt genau }}$ \\
\hline $\begin{array}{l}\text { Wenn ich von einer Sache abgelenkt werde, komme ich schnell } \\
\text { wieder zum Thema zurück. }\end{array}$ & 0 & 0 & 0 & O \\
\hline $\begin{array}{l}\text { Wenn störende Gedanken auftreten, kann ich sie nur schwer von } \\
\text { mir weg schieben. }\end{array}$ & 0 & O & $\mathrm{O}$ & 0 \\
\hline $\begin{array}{l}\text { Bei der Bearbeitung einer schwierigen Aufgabe, führe ich mir vor } \\
\text { Augen, was ich schon alles geschafft habe. }\end{array}$ & O & 0 & O & O \\
\hline Beim Lernen bin ich unkonzentriert. & 0 & O & 0 & O \\
\hline Beim Lernen merke ich, dass meine Gedanken abschweifen. & O & 0 & O & O \\
\hline Es fällt mir schwer, mich aufs Lernen zu konzentrieren. & 0 & 0 & 0 & 0 \\
\hline $\begin{array}{l}\text { Gewöhnlich dauert es nicht lange, bis ich mich dazu entschließe, } \\
\text { mit dem Lernen anzufangen. }\end{array}$ & 0 & 0 & 0 & 0 \\
\hline $\begin{array}{l}\text { Ich freue mich auf eine Stunde im Fach Naturwissenschaften } \\
\text { (NW). }\end{array}$ & 0 & 0 & 0 & 0 \\
\hline Ich kann mich nicht lange konzentrieren. & 0 & 0 & 0 & O \\
\hline $\begin{array}{l}\text { Ich lerne für die Schule, weil ich bei den Klassenarbeiten möglichst } \\
\text { gut abschneiden möchte. }\end{array}$ & 0 & O & 0 & 0 \\
\hline Ich lerne für die Schule, weil ich erfolgreich sein möchte. & 0 & 0 & O & O \\
\hline $\begin{array}{l}\text { Ich versuche mich dazu zu bringen, die Hausaufgaben zu erledi- } \\
\text { gen, auch wenn Schwierigkeiten auftreten. }\end{array}$ & 0 & 0 & 0 & 0 \\
\hline $\begin{array}{l}\text { Ich versuche mich dazu zu bringen, Hausaufgaben zu erledigen, } \\
\text { auch wenn ich keine Lust habe. }\end{array}$ & 0 & 0 & 0 & 0 \\
\hline $\begin{array}{l}\text { Mir ist es wichtig, im Fach Naturwissenschaften (NW) viel zu wis- } \\
\text { sen. }\end{array}$ & 0 & 0 & 0 & 0 \\
\hline $\begin{array}{l}\text { Nach einem Misserfolg komme ich oft über eine ganze Zeit nicht } \\
\text { mehr aus dem Grübeln heraus. }\end{array}$ & 0 & 0 & 0 & 0 \\
\hline Ich lerne für die Schule, weil ich gute Leistungen zeigen möchte. & 0 & 0 & 0 & O \\
\hline $\begin{array}{l}\text { Nach einem Misserfolg muss ich lange darüber nachdenken, wie } \\
\text { es dazu kommen konnte, bevor ich mich auf etwas anderes kon- } \\
\text { zentrieren kann. }\end{array}$ & 0 & 0 & 0 & 0 \\
\hline Schwierige Aufgaben schiebe ich oft lange vor mir her. & 0 & 0 & O & O \\
\hline $\begin{array}{l}\text { Traurige Gedanken werde ich schlecht wieder los, wenn sie einmal } \\
\text { da sind. }\end{array}$ & 0 & 0 & 0 & 0 \\
\hline $\begin{array}{l}\text { Wenn ich bei der Bearbeitung einer Aufgabe die Lust verliere, } \\
\text { überlege ich mir etwas, das mich wieder zum Arbeiten bringt. }\end{array}$ & 0 & 0 & O & 0 \\
\hline
\end{tabular}




\begin{tabular}{|c|c|c|c|c|}
\hline & $\underset{\substack{\text { stimmt gar } \\
\text { nicht }}}{\downarrow}$ & $\begin{array}{l}\text { stimmt eher } \\
\text { nicht } \\
\downarrow\end{array}$ & $\underset{\downarrow}{\operatorname{stimmt} \text { eher }}$ & $\underset{\downarrow}{\operatorname{stimmt~genau}}$ \\
\hline $\begin{array}{l}\text { Wenn ich in eine schlechte Stimmung gerate, komme ich da ganz } \\
\text { schwer wieder heraus. }\end{array}$ & $\mathrm{O}$ & O & $\mathrm{O}$ & $\mathrm{O}$ \\
\hline $\begin{array}{l}\text { Wenn ich keine Lust habe, eine Aufgabe zu bearbeiten, lasse ich } \\
\text { es bleiben. }\end{array}$ & O & O & O & O \\
\hline $\begin{array}{l}\text { Wenn ich mich besonders angestrengt habe, lobt mich der Lehrer } \\
\text { meistens, auch wenn andere Schüler noch besser sind als ich. }\end{array}$ & O & O & O & $\mathrm{O}$ \\
\hline $\begin{array}{l}\text { Ich lerne für die Schule, weil ich sie erfolgreich abschließen möch- } \\
\text { te. }\end{array}$ & 0 & 0 & 0 & 0 \\
\hline $\begin{array}{l}\text { Auch wenn meine Hausaufgaben langweilig und eintönig sind, } \\
\text { höre ich erst auf, wenn ich fertig bin. }\end{array}$ & O & O & $\mathrm{O}$ & $\mathrm{O}$ \\
\hline $\begin{array}{l}\text { Bei einem neuen Thema stelle ich mir praktische Anwendungs- } \\
\text { möglichkeiten vor. }\end{array}$ & 0 & O & O & O \\
\hline $\begin{array}{l}\text { Bei Hausaufgaben versuche ich immer, so gründlich wie möglich } \\
\text { zu arbeiten. }\end{array}$ & 0 & O & O & 0 \\
\hline Ich beziehe das, was ich lerne, auf meine eigenen Erfahrungen. & 0 & O & O & O \\
\hline $\begin{array}{l}\text { Ich denke darüber nach, was ich an meinem Lernverhalten ändern } \\
\text { könnte. }\end{array}$ & 0 & $\mathrm{O}$ & 0 & $\mathrm{O}$ \\
\hline $\begin{array}{l}\text { Wenn sich ein schwacher Schüler verbessert, ist das für unseren } \\
\text { Lehrer eine gute Leistung, auch wenn der Schüler immer noch } \\
\text { unter dem Klassendurchschnitt liegt. }\end{array}$ & 0 & 0 & O & 0 \\
\hline Ich denke mir konkrete Beispiele zu bestimmten Lerninhalten aus. & 0 & O & $\mathrm{O}$ & O \\
\hline Wenn ich etwas Neues mache, weiß ich, wie ich damit umgehe. & 0 & O & 0 & O \\
\hline $\begin{array}{l}\text { Ich denke oft darüber nach, wie ich mich in bestimmten Situationen } \\
\text { anders verhalten könnte. }\end{array}$ & 0 & O & O & O \\
\hline Ich gebe nicht auf, auch wenn die Aufgabe sehr schwierig ist. & O & O & O & O \\
\hline Ich lege vor dem Lernen fest, wie weit ich kommen möchte. & O & 0 & $\mathrm{O}$ & 0 \\
\hline $\begin{array}{l}\text { Ich lerne so lange, bis ich mir sicher bin, eine Klassenarbeit gut } \\
\text { bestehen zu können. }\end{array}$ & 0 & 0 & 0 & 0 \\
\hline Ich mache meine Hausaufgaben so gut wie möglich. & 0 & 0 & 0 & 0 \\
\hline Ich stelle mir manche Sachverhalte bildlich vor. & 0 & 0 & 0 & 0 \\
\hline $\begin{array}{l}\text { Ich strenge mich auch dann an, wenn mir das Fach überhaupt } \\
\text { nicht liegt. }\end{array}$ & 0 & 0 & 0 & 0 \\
\hline $\begin{array}{l}\text { Ich überlege mir vorher, in welcher Reihenfolge ich die Hausauf- } \\
\text { gaben durcharbeite. }\end{array}$ & O & O & 0 & 0 \\
\hline
\end{tabular}




\begin{tabular}{|c|c|c|c|c|}
\hline & $\begin{array}{c}\text { stimmt gar } \\
\text { nicht } \\
\downarrow\end{array}$ & $\begin{array}{c}\text { stimmt eher } \\
\text { nicht } \\
\downarrow\end{array}$ & $\underset{\downarrow}{\operatorname{stimmt} \text { eher }}$ & $\underset{\downarrow}{\text { stimmt genau }}$ \\
\hline $\begin{array}{l}\text { Ich versuche das Gelernte mit dem zu verbinden, was ich schon } \\
\text { darüber weiß. }\end{array}$ & 0 & 0 & 0 & O \\
\hline Für jedes Problem habe ich eine Lösung. & O & 0 & O & O \\
\hline $\begin{array}{l}\text { Ich versuche, mir vor einer Klassenarbeit genau zu überlegen, was } \\
\text { ich lernen muss und was nicht. }\end{array}$ & O & 0 & 0 & 0 \\
\hline $\begin{array}{l}\text { Mir liegt viel daran, den Stoff des Faches Naturwissenschaften } \\
\text { (NW) zu behalten. }\end{array}$ & 0 & 0 & 0 & O \\
\hline $\begin{array}{l}\text { Vor dem Lernen überlege ich mir, wie ich am besten vorgehen } \\
\text { kann. }\end{array}$ & O & 0 & O & O \\
\hline $\begin{array}{l}\text { Vor einer Klassenarbeit nehme ich mir genug Zeit, um den ganzen } \\
\text { Stoff noch einmal durchzugehen. }\end{array}$ & O & 0 & 0 & 0 \\
\hline $\begin{array}{l}\text { Unser Lehrer lobt auch die schlechten Schüler, wenn er merkt, } \\
\text { dass sie sich verbessern. }\end{array}$ & 0 & 0 & 0 & 0 \\
\hline $\begin{array}{l}\text { Beim Lösen einer Aufgabe achte ich darauf, ob ich mich noch } \\
\text { konzentriere. }\end{array}$ & 0 & 0 & 0 & 0 \\
\hline $\begin{array}{l}\text { Die Lösung schwieriger Probleme gelingt mir immer, wenn ich } \\
\text { mich darum bemühe. }\end{array}$ & 0 & 0 & 0 & 0 \\
\hline $\begin{array}{l}\text { Die wichtigsten Unterlagen habe ich an meinem Arbeitsplatz griff- } \\
\text { bereit. }\end{array}$ & 0 & O & 0 & 0 \\
\hline $\begin{array}{l}\text { Es bereitet mir keine Schwierigkeiten, meine Absichten und Ziele } \\
\text { zu verwirklichen. }\end{array}$ & 0 & 0 & 0 & 0 \\
\hline Fehler zu machen ist nutzlos. & 0 & 0 & 0 & 0 \\
\hline $\begin{array}{l}\text { Ich denke bei den Hausaufgaben darüber nach, ob ich die Aufga- } \\
\text { ben auch richtig verstanden habe. }\end{array}$ & 0 & 0 & 0 & 0 \\
\hline $\begin{array}{l}\text { Wenn viele Probleme auf mich zukommen, dann kann ich mich } \\
\text { nicht mehr konzentrieren. }\end{array}$ & 0 & 0 & 0 & 0 \\
\hline $\begin{array}{l}\text { Ich finde es oft schwierig einzuschätzen, wie weit ich von meinem } \\
\text { Ziel noch entfernt bin. }\end{array}$ & 0 & 0 & 0 & 0 \\
\hline $\begin{array}{l}\text { Ich gestalte meine Umgebung so, dass ich möglichst wenig vom } \\
\text { Lernen abgelenkt werde. }\end{array}$ & 0 & O & 0 & 0 \\
\hline $\begin{array}{l}\text { Ich lerne an einem Platz, wo ich mich gut auf den Lernstoff kon- } \\
\text { zentrieren kann. }\end{array}$ & 0 & 0 & 0 & 0 \\
\hline Ich überlege mir nach einem Fehler, was ich verändern kann. & 0 & 0 & 0 & 0 \\
\hline Mein Lernplatz ist so gestaltet, dass ich alles schnell finden kann. & $\mathrm{O}$ & $\mathrm{O}$ & O & $\mathrm{O}$ \\
\hline Fehler zeigen mir, was ich anders machen könnte. & 0 & 0 & 0 & 0 \\
\hline
\end{tabular}




\begin{tabular}{|c|c|c|c|c|}
\hline & $\underset{\substack{\text { stimmt gar } \\
\text { nicht }}}{\downarrow}$ & $\begin{array}{l}\text { stimmt eher } \\
\text { nicht } \\
\downarrow\end{array}$ & $\underset{\downarrow}{\operatorname{stimmt} \text { eher }}$ & $\underset{\downarrow}{\operatorname{stimmt~genau}}$ \\
\hline $\begin{array}{l}\text { Während ich auf ein Ziel hinarbeite, überprüfe ich, ob mein Vorge- } \\
\text { hen mich dem Ziel näher bringt. }\end{array}$ & $\mathrm{O}$ & O & O & $\mathrm{O}$ \\
\hline Wenn eine Sache kompliziert wird, gebe ich auf. & $\mathrm{O}$ & $\mathrm{O}$ & O & $\mathrm{O}$ \\
\hline $\begin{array}{l}\text { Wenn ich auf ein Ziel hinarbeite, überprüfe ich währenddessen, } \\
\text { wie weit ich bisher gekommen bin. }\end{array}$ & 0 & O & 0 & 0 \\
\hline Fehler versuche ich schnell wieder zu vergessen. & O & O & O & O \\
\hline $\begin{array}{l}\text { Wenn ich ein Ziel noch nicht erreicht habe, dann mache ich mir } \\
\text { den momentanen Stand bewusst. }\end{array}$ & 0 & O & 0 & 0 \\
\hline $\begin{array}{l}\text { Wenn ich etwas noch nicht kann, dann übe ich mit Geduld und } \\
\text { Ausdauer. }\end{array}$ & O & O & O & O \\
\hline Wenn ich lerne, sorge ich dafür, dass ich in Ruhe arbeiten kann. & O & 0 & 0 & 0 \\
\hline $\begin{array}{l}\text { Wenn ich nicht weiterkomme, kenne ich bestimmte Fragen, die mir } \\
\text { weiterhelfen können. }\end{array}$ & O & $\mathrm{O}$ & O & O \\
\hline $\begin{array}{l}\text { Wenn sich mir etwas in den Weg stellt, gebe ich mir die größte } \\
\text { Mühe, es zu überwinden. }\end{array}$ & 0 & 0 & 0 & 0 \\
\hline Zum Lernen sitze ich immer am selben Platz. & O & O & $\mathrm{O}$ & O \\
\hline $\begin{array}{l}\text { Auch schwierige Aufgaben stellen für mich keine unüberwindbaren } \\
\text { Hindernisse dar. }\end{array}$ & 0 & 0 & 0 & 0 \\
\hline $\begin{array}{l}\text { Wenn ein Schüler seine Leistungen verbessert, wird er vom Lehrer } \\
\text { gelobt, auch dann, wenn er im Vergleich zur Klasse unter dem } \\
\text { Durchschnitt liegt. }\end{array}$ & 0 & O & O & O \\
\hline Ich denke häufig über mein Lernverhalten nach. & 0 & O & O & 0 \\
\hline $\begin{array}{l}\text { Wenn es einmal Probleme gibt, dann werde ich sie schon bewälti- } \\
\text { gen. }\end{array}$ & 0 & 0 & 0 & 0 \\
\hline $\begin{array}{l}\text { Wenn ich bei den Hausaufgaben eine Aufgabe nicht gleich verste- } \\
\text { he, gebe ich mir immer viel Mühe, sie doch noch zu verstehen. }\end{array}$ & 0 & 0 & 0 & 0 \\
\hline $\begin{array}{l}\text { Wenn ich mir etwas zu Lernen vorgenommen habe, bemühe ich } \\
\text { mich, es auch zu schaffen. }\end{array}$ & 0 & 0 & 0 & 0 \\
\hline $\begin{array}{l}\text { Wenn ich etwas mache, kann ich mich immer gut darauf konzent- } \\
\text { rieren. }\end{array}$ & 0 & 0 & 0 & 0 \\
\hline $\begin{array}{l}\text { Wenn ich mit einem Problem konfrontiert werde, habe ich meist } \\
\text { mehrere Ideen, wie ich damit fertig werde. }\end{array}$ & 0 & 0 & 0 & 0 \\
\hline Wenn ich lerne, bin ich leicht abzulenken. & 0 & 0 & 0 & 0 \\
\hline
\end{tabular}

Nun hast Du es geschafft! Vielen Dank für deine Mitarbeit 
Anhang B

Instruktion für die experimentellen Gruppen in Studie 2 


\section{Standards Instruktion}

\section{Mastery Learning}

Du bekommst gleich noch einige dieser Aufgaben.

Ich weiß, dass diese Aufgabe wahrscheinlich ziemlich neu für dich ist und vielleicht auch recht knifflig. Aber deshalb übt man sie ja.

Alle Kinder können lernen sie zu lösen. Auch wenn man es vorher noch nicht konnte, kann man das schaffen.

Es ist schon gut, wenn man sich Schritt für Schritt selbst verbessert und lernt, diese Aufgabe zu lösen.

\section{$\underline{\text { Sozialer Vergleich }}$}

Du bekommst gleich noch einige dieser Aufgaben.

Ich weiß, dass diese Aufgabe wahrscheinlich ziemlich neu für dich ist und vielleicht auch recht knifflig.

Manche Kinder sind richtig gut beim Lösen dieser Aufgabe, manche Kinder sind nicht so gut. Wir schauen mal, wie gut Du bist! 


\section{Feedback Instruktion}

Individuell

Jetzt kommen also noch einige dieser Aufgaben. Auf jedem Blatt sind 5 Aufgaben, man kann also immer 5 Punkte bekommen, wenn man alles richtig macht.

Nach jeder Aufgabe sage ich dir, wie viele Punkte du bekommen hast.

Deine Punktzahl zeichne ich hier an der Tafel ein.

\section{TAFEL ZEIGEN}

Auf der Linie hier unten stehen die Nummern der Durchgänge, also 1. Durchgang, 2. Durchgang, 3. Durchgang, und so weiter.

\section{ZEIGEN}

Auf diesem Pfeil, der nach oben zeigt, steht die Punktzahl, also 1 Punkt, 2 Punkte, und so weiter, bis 5 Punkte.

\section{ZEIGEN}

Wenn du zum Beispiel in der ersten Aufgabe 2 Punkte bekommst, zeichnen wir das hier ein.

PUNKT MARKIEREN.

Wenn du in der zweiten Aufgabe 3 Punkte erreichen würdest, wo müsste ich dann den Punkt hinmalen?

\section{ZEIGEN LASSEN, DANN PUNKT MARKIEREN}

Hast du das verstanden? 


\section{$\underline{\text { Sozialer Vergleich }}$}

Jetzt kommen also noch einige dieser Aufgaben. Auf jedem Blatt sind 5 Aufgaben, man kann also immer 5 Punkte bekommen, wenn man alles richtig macht. Nach jeder Aufgabe sage ich dir, wie viele Punkte du bekommen hast.

Deine Punktzahl zeichne ich hier an der Tafel ein.

\section{TAFEL ZEIGEN}

Auf der Linie hier unten stehen die Nummern der Durchgänge, also 1. Durchgang, 2. Durchgang, 3. Durchgang, und so weiter.

\section{ZEIGEN}

Auf diesem Pfeil, der nach oben zeigt, steht die Punktzahl, also 1 Punkt, 2 Punkte, und so weiter, bis 5 Punkte.

\section{ZEIGEN}

Wenn du zum Beispiel in der ersten Aufgabe 2 Punkte bekommst, zeichnen wir das hier ein.

\section{PUNKT MARKIEREN.}

Wenn du in der zweiten Aufgabe 3 Punkte erreichen würdest, wo müsste ich dann den Punkt hinmalen?

\section{ZEIGEN LASSEN, DANN PUNKT MARKIEREN}

Dann sage ich dir noch, wie viele Punkte die meisten anderen Kinder in dieser Aufgabe bekommen haben. Diese Punktzahl zeichne ich hier mit einer anderen Farbe an der Tafel ein.

Wenn die meisten anderen Kinder hier 3 Punkte bekommen haben, zeichne ich das hier ein.

\section{PUNKT MARKIEREN, ANDERE FARBE}

Und wenn die meisten anderen Kinder 2 Punkte erreichen würden, wo müsste ich dann den anderen Punkt hinmalen?

\section{ZEIGEN LASSEN, DANN PUNKT MARKIEREN}

Hast du das verstanden? 
Anhang C

Messinstrument von Studie 2 


\begin{tabular}{|l|}
\hline Datum \\
\hline Gruppe $\quad \mathrm{S}$ \\
\hline Name
\end{tabular}

\begin{tabular}{|l|l|}
\hline Klasse & \\
\hline Alter & \\
\hline Geschlecht & $\square$ Mädchen $\square$ Junge \\
\hline Land & \\
\hline Lieblingsfach & \\
\hline $\begin{array}{l}\text { Mathenote } \\
\text { (letztes Zeugnis) }\end{array}$ & \\
\hline
\end{tabular}


1. Schaue dir die Aufgaben auf der nächsten Seite an. Zunächst einmal nur anschauen!

2. Beantworte dann die Fragen

3. Wenn du die Fragen beantwortet hast, bearbeite die Aufgaben auf der nächsten Seite!

Wie sicher bist du dir, dass du diese Aufgaben richtig lösen

$$
\begin{array}{r}
\text { überhaupt } \\
\text { nicht } \\
\text { sicher }
\end{array}
$$
(1)
(2)
(3)
(4)
(5)
(6)
(7)
(8)
(9)

überhaupt
nicht
interes-
sant

2. Wie interessant findest du diese Aufgabe im Vergleich zu anderen Matheaufgaben, die Du schon gerechnet hast?
(1)
(2)
(3)
(4)
(5)
(6)
(7) (8)
(9)

sehr interessant schon gerechnet hast?

3. Glaubst Du, dass die Fähigkeit,
solche Aufgaben zu lösen, wichtig ist, um gut in der Schule zu sein?

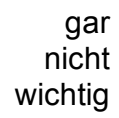

(1)

(2)

(3)

(4)

(5)

(6)

(7)

(8)

sehr

wichtig

Wenn Du die Fragen beantwortet hast, bearbeite bitte die Aufgaben auf der nächsten Seite! 


\section{AUFGABE:}

a)

$(51+37)-9 \cdot 6$

b)

$(49-43) \cdot 3+7$

c)

$8 \cdot 7+5+(43+29)$

d)

$41+(76-47)+7 \cdot 6$ e)

$(73-44)+6 \cdot 6+13$

f)

$6 \cdot 7+(86-37)$

g)

$3+(91-55)+3 \cdot 9$

h)

$(36+28)-8 \cdot 4-3$

Wenn Du mit dem Rechnen fertig bist, beantworte diese Frage:

\section{überhaupt}

nicht si-

4. Wie sicher bist du dir, dass du die Aufgaben richtig gelöst hast?

cher

sehr sicher

(1) (2) (3) (4) (5) (6) (7) (8) (9)

5. Schätze, wie viele von den 8

(0)

(1) (2)

(3)

(4)

(5)

(6) 7

(8) 
1. Schaue dir die Aufgaben weiter unten auf diesem Blatt an. Zunächst einmal nur anschauen!

2. Beantworte die Fragen 1 und 2!

3. Wenn du die Fragen beantwortet hast, bearbeite die Aufgaben!

4. Wenn du mit dem Rechnen fertig bist, beantworte die Fragen 3 und 4 !

\section{überhaupt}

nicht si-

cher sehr sicher

1. Wie sicher bist du dir, dass du diese Aufgaben richtig lösen

(1)

(2)

(3)

(4)

(5)

(6)

(7) 8

(9)

kannst?

2. Schätze, wie viele von den 8 Aufgaben du richtig lösen

(0)

(1)

(2)

(3) (4)

(4) (5)

(6)

(7)

(8)

\section{AUFGABE}

a)

$(43-24)+8 \cdot 6-5$

b)

$8 \cdot(73-56)+7$

c)

$100-(25-13)+5 \cdot 6$

d)

$(74+24)-9 \cdot 8+15$ e)

$(82+19)-1+8 \cdot 7$

f)

$9 \cdot 4+11-(23+21)$

g)

$6 \cdot 8+3+(77-52)$

h)

$2+6 \cdot 4+(52+69)$

überhaupt

nicht si-

cher

sehr sicher

3. Wie sicher bist du dir, dass du

(1) (2)

(3)

(4)

(5)

(6)

(8)

(9)

4. Schätze, wie viele von den 8 Aufgaben du richtig gelöst hast!

(0)

(1)

(2)

(3)

(4)

(5)

(6) (7)

(8) 
Protokollbogen für Versuchsleiterln

Bitte so genau wie möglich Notizen machen, auch wenn Video läuft

\begin{tabular}{|l|l|l|}
\hline 1. & $\begin{array}{l}\text { \&ündlich, } \\
\text { Antwort aufzeichnen }\end{array}$ & Wie bist Du beim Rechnen vorgegangen? \\
\hline & $\begin{array}{l}\text { (falls Kinds sagt, „so wie Sie es erklärt haben“, nachfragen: „kannst du mir das nochmal } \\
\text { sagen?") }\end{array}$ \\
\hline & \begin{tabular}{l} 
Nachfrage: Hattest Du einen bestimmten Trick oder Lösungsweg? \\
\hline
\end{tabular} \\
\hline
\end{tabular}

\begin{tabular}{|l|l|l|}
\hline 2. & $\begin{array}{l}\text { \& mündlich, } \\
\text { Antwort aufzeichnen }\end{array}$ & $\begin{array}{l}\text { Was meinst du, warum du manchmal Fehler gemacht hast? } \\
\text { (kann entfallen, falls Kind ALLE Aufgaben richtig gelöst hat) }\end{array}$ \\
& $\begin{array}{l}\text { (falls Kind aus Feedback KG nachfragt, ob es Fehler gemacht hat: „Sagen wir mal, das wäre } \\
\text { Was kann man bei dieser Aufgabe falsch machen? }\end{array}$ \\
\hline
\end{tabular}

\begin{tabular}{|l|l|l|}
\hline 3. Antwort notieren & Wenn du solche Aufgaben nochmal rechnen musst, würdest du beim nächs-
\end{tabular} ten Mal etwas anders machen?

O JA O NEIN

(†) mündlich,
Antwort aufzeichnen

Wenn ja, was? 
Zum Schluss noch einige Fragen:

überhaupt

nicht

sehr viel

1. Hast du, während du die Aufgaben gelöst hast, darüber

nachgedacht, ob du auf dem

(1) (2)

(3)

(4)

(5)

(6)

(7)

(8)

(9) richtigen Weg bist?

\section{überhaupt \\ nicht gut}

2. Wie bewertest Du selbst Deine Leistung in den Aufgaben, die du jetzt gelöst hast?
(1)
(2)
(3)
(4) (5)
(6)
(7)
(8)
(9)

sehr gut

3. Warum bewertest du deine Leistung so?

\section{überhaupt \\ nicht zu- \\ frieden}

4. Wie zufrieden bist du mit deiner Leistung?

(1)

(2)

(3)

(4)

(5)

(6)

frieden

(9) 
Anhang D

Aufgaben der vier Übungsdurchgänge in Studie 2 
AUFGABE 1

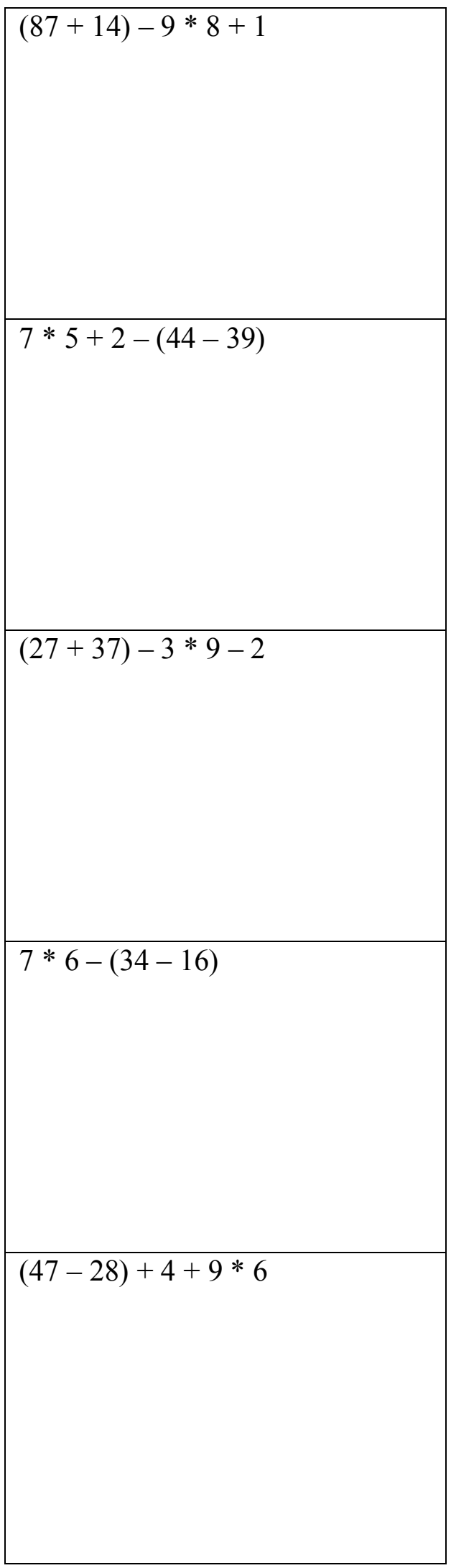


AUFGABE 2

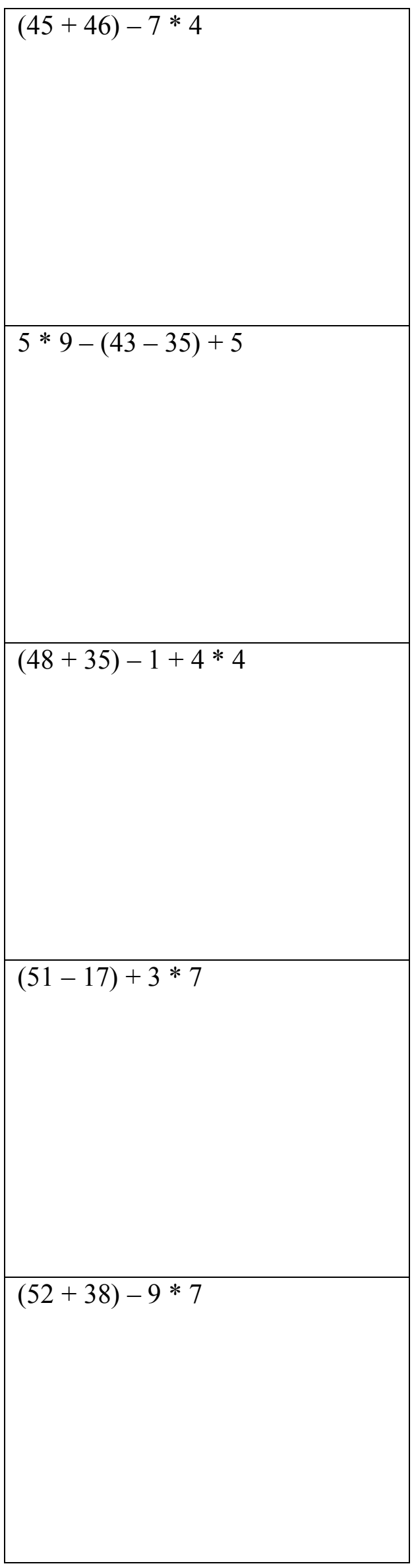

188 
AUFGABE 3

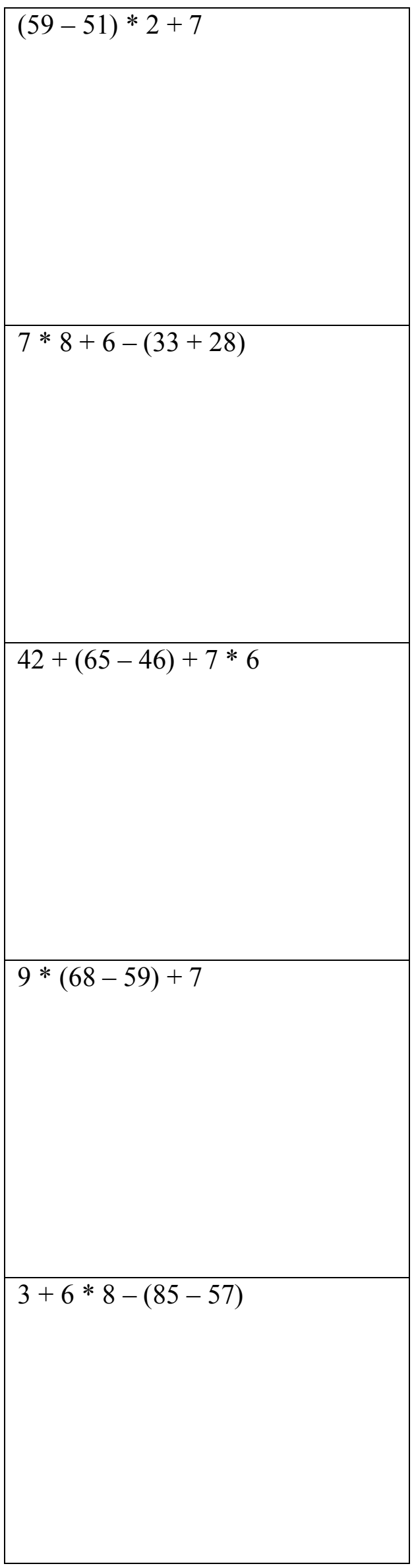


AUFGABE 4

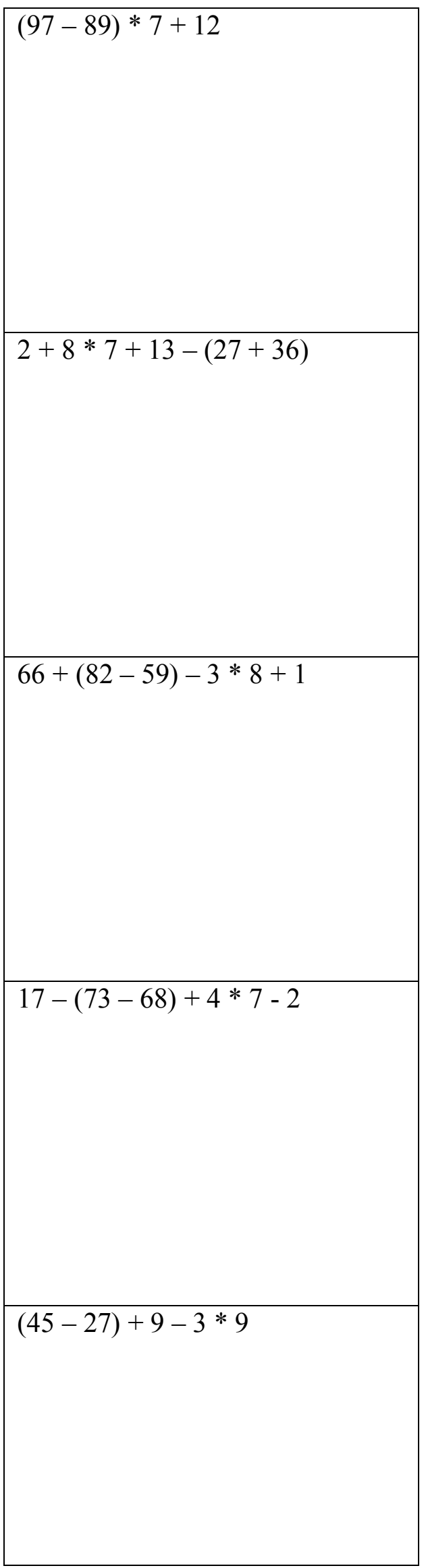




\section{LEBENSLAUF}

\begin{aligned} & Name Andju Sara Labuhn \\ & Nationalität deutsch \\ & Geburtsdatum 8. Juni 1980 \\ & Anschrift Kapitän-Lehmann-Str. 6, 37083 Göttingen \\ & Telefon 05512814134 \\ & AUSBILDUNG \\ & \hline A999 \\ & Oktober 2004 Gesamtschule Kierspe \\ & Diplom in Psychologie, Philipps-Universität Marburg \\ & seit Oktober 2005 \\ & Promotionsstipendium im DFG-Graduiertenkolleg “Passungsverhältnisse \\ & schulischen Lernens”, Georg-August Universität Göttingen \\ & FORSCHUNGSERFAHRUNG \end{aligned}

\section{$2002-2004$}

Studentische Hilfskraft in der Arbeitsgruppe Sozialpsychologie, Philipps-Universität Marburg

\section{Februar 2002 - April 2002}

Forschungspraktikum in der Arbeitsgruppe Sozialpsychologie, Philipps-Universität Marburg

\section{September 2002 - Oktober 2002}

Forschungspraktikum in der Arbeitsgruppe Gesundheits- und Gemeindepsychologie an der Università di Bologna, Italien

\section{Oktober 2003}

Forschungsaufenthalt an der Aston Business School, Birmingham, England

Februar 2007 - Mai 2007

Forschungsaufenthalt an der City University of New York, PhD Program in Educational Psychology, New York, USA, bei Prof. Dr. B.J. Zimmerman

\section{Oktober 2007 - Dezember 2007}

Forschungsaufenthalt an der City University of New York, PhD Program in Educational Psychology, New York, USA, bei Prof. Dr. B.J. Zimmerman

\section{WeITERe Praktika}

April 2003 - Juli 2003
Schulpsychologische Beratungsstelle, Marburg
Durchführung des "Marburger Konzentrationstrainings"
April 2003 - Juli 2003
Kinderklinik Marburg und KfH Kuratorium für Dialyse und Nierentrans-
plantation e.V., Nierenzentrum für Kinder und Jugendliche

Englisch, Basiskenntnisse Italienisch und Latein 